\title{
IPCC
}

INTERGOVERNMENTAL PANEL ON Climate chanழe

\section{CLIMATE CHANGE 2014 \\ Mitigation of Climate Change \\ GE 2014}

\section{Summary for Policymakers and Technical Summary}




\section{Climate Change 2014 \\ Mitigation of Climate Change}

Summary for Policymakers

Technical Summary

\section{Part of the Working Group III Contribution to the Fifth Assessment Report of the Intergovernmental Panel on Climate Change}

\begin{tabular}{|c|c|c|}
\hline \multicolumn{3}{|c|}{ Edited by } \\
\hline $\begin{array}{l}\text { Ottmar Edenhofer } \\
\text { Working Group III Co-Chair } \\
\text { Potsdam Institute for } \\
\text { Climate Impact Research }\end{array}$ & $\begin{array}{c}\text { Ramón Pichs-Madruga } \\
\text { Working Group III Co-Chair } \\
\text { Centro de Investigaciones de la } \\
\text { Economía Mundial }\end{array}$ & $\begin{array}{c}\text { Youba Sokona } \\
\text { Working Group III Co-Chair } \\
\text { South Centre }\end{array}$ \\
\hline $\begin{array}{l}\text { Jan C. Minx } \\
\text { Head of TSU }\end{array}$ & $\begin{array}{c}\text { Ellie Farahani } \\
\text { Head of Operations }\end{array}$ & $\begin{array}{c}\text { Kristin Seyboth } \\
\text { Deputy Head of Science }\end{array}$ \\
\hline $\begin{array}{c}\text { Anna Adler } \\
\text { Team Assistant }\end{array}$ & $\begin{array}{c}\text { Ina Baum } \\
\text { Project Officer }\end{array}$ & $\begin{array}{l}\text { Steffen Brunner } \\
\text { Senior Economist }\end{array}$ \\
\hline $\begin{array}{l}\text { Patrick Eickemeier } \\
\text { Scientific Editor }\end{array}$ & $\begin{array}{c}\text { Benjamin Kriemann } \\
\text { IT Officer }\end{array}$ & $\begin{array}{l}\text { Jussi Savolainen } \\
\text { Web Manager }\end{array}$ \\
\hline $\begin{array}{l}\text { Steffen Schlömer } \\
\text { Scientist }\end{array}$ & $\begin{array}{c}\text { Christoph von Stechow } \\
\text { Scientist }\end{array}$ & $\begin{array}{l}\text { Timm Zwickel } \\
\text { Senior Scientist }\end{array}$ \\
\hline
\end{tabular}

Working Group III Technical Support Unit 
(C) Intergovernmental Panel on Climate Change 2015

ISBN 978-92-9169-142-5

Figure SPM. 4 as originally included in the digital version of this publication contained an error. This error is now corrected in this publication after having completed, in January 2015, the relevant procedures under the IPCC Protocol for Addressing Errors in IPCC Assessment Reports, Synthesis Reports, Special Reports or Methodological Reports.

The designations employed and the presentation of material on maps do not imply the expression of any opinion whatsoever on the part of the Intergovernmental Panel on Climate Change concerning the legal status of any country, territory, city or area or of its authorities, or concerning the delimitation of its frontiers or boundaries.

Cover photo:

Shanghai, China, aerial view @) Ocean/Corbis

Dedication photo:

Elinor Ostrom (c) dpa 
Foreword, Preface,

Dedication and

In Memoriam 



\section{Foreword}

Climate Change 2014: Mitigation of Climate Change is the third part of the Fifth Assessment Report (AR5) of the Intergovernmental Panel on Climate Change (IPCC)_Climate Change 2013/2014_and was prepared by its Working Group III. The volume provides a comprehensive and transparent assessment of relevant options for mitigating climate change through limiting or preventing greenhouse gas (GHG) emissions, as well as activities that reduce their concentrations in the atmosphere.

This report highlights that despite a growing number of mitigation policies, GHG emission growth has accelerated over the last decade. The evidence from hundreds of new mitigation scenarios suggests that stabilizing temperature increase within the $21^{\text {st }}$ century requires a fundamental departure from business-as-usual. At the same time, it shows that a variety of emission pathways exists where the temperature increase can be limited to below $2^{\circ} \mathrm{C}$ relative to pre-industrial level. But this goal is associated with considerable technological, economic and institutional challenges. A delay in mitigation efforts or the limited availability of low carbon technologies further increases these challenges. Less ambitious mitigation goals such as $2.5^{\circ} \mathrm{C}$ or $3^{\circ} \mathrm{C}$ involve similar challenges, but on a slower timescale. Complementing these insights, the report provides a comprehensive assessment of the technical and behavioural mitigation options available in the energy, transport, buildings, industry and land-use sectors and evaluates policy options across governance levels from the local to the international scale.

The findings in this report have considerably enhanced our understanding of the range of mitigation pathways available and their underlying technological, economic and institutional requirements. The timing of this report is thus critical, as it can provide crucial information for the negotiators responsible for concluding a new agreement under the United Nations Framework Convention on Climate Change in 2015. The report therefore demands the urgent attention of both policymakers and the general public.

As an intergovernmental body jointly established in 1988 by the World Meteorological Organization (WMO) and the United Nations Environment Programme (UNEP), the IPCC has successfully provided policymakers with the most authoritative and objective scientific and technical assessments, which are clearly policy relevant without being policy prescriptive. Beginning in 1990, this series of IPCC Assessment Reports, Special Reports, Technical Papers, Methodology Reports and other products have become standard works of reference.

This Working Group III assessment was made possible thanks to the commitment and dedication of many hundreds of experts, representing a wide range of regions and scientific disciplines. WMO and UNEP are proud that so many of the experts belong to their communities and networks.
We express our deep gratitude to all authors, review editors and expert reviewers for devoting their knowledge, expertise and time. We would like to thank the staff of the Working Group III Technical Support Unit and the IPCC Secretariat for their dedication.

We are also thankful to the governments that supported their scientists' participation in developing this report and that contributed to the IPCC Trust Fund to provide for the essential participation of experts from developing countries and countries with economies in transition.

We would like to express our appreciation to the government of Italy for hosting the scoping meeting for the IPCC's Fifth Assessment Report, to the governments of Republic of Korea, New Zealand and Ethiopia as well as the University of Vigo and the Economics for Energy Research Centre in Spain for hosting drafting sessions of the Working Group III contribution and to the government of Germany for hosting the Twelfth Session of Working Group III in Berlin for approval of the Working Group III Report. In addition, we would like to thank the governments of India, Peru, Ghana, the United States and Germany for hosting the AR5 Expert meetings in Calcutta, Lima, Accra, Washington D.C., and Potsdam, respectively. The generous financial support by the government of Germany, and the logistical support by the Potsdam Institute for Climate Impact Research (Germany), enabled the effective operation of the Working Group III Technical Support Unit. This is gratefully acknowledged.

We would particularly like to thank Dr. Rajendra Pachauri, Chairman of the IPCC, for his direction and guidance of the IPCC and we express our deep gratitude to Professor Ottmar Edenhofer, Dr. Ramon PichsMadruga, and Dr. Youba Sokona, the Co-Chairs of Working Group III for their tireless leadership throughout the development and production of this report.

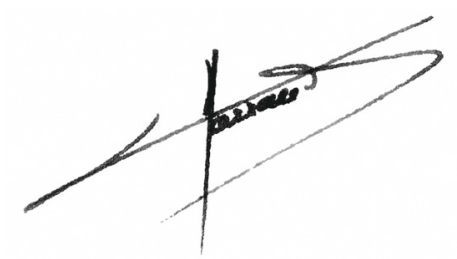

M. Jarraud

Secretary-General

World Meteorological Organization

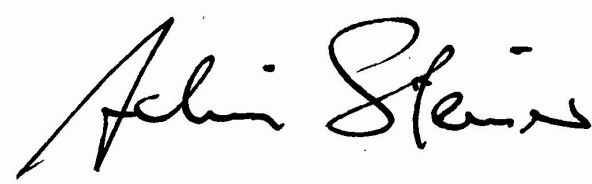
A. Steiner
Executive Director
United Nations Environment Programme 



\section{Preface}

The Working Group III contribution to the Fifth Assessment Report (AR5) of the Intergovernmental Panel on Climate Change (IPCC) provides a comprehensive and transparent assessment of the scientific literature on climate change mitigation. It builds upon the Working Group III contribution to the IPCC's Fourth Assessment Report (AR4) in 2007, the Special Report on Renewable Energy Sources and Climate Change Mitigation (SRREN) in 2011 and previous reports and incorporates subsequent new findings and research. The report assesses mitigation options at different levels of governance and in different economic sectors. It evaluates the societal implications of different mitigation policies, but does not recommend any particular option for mitigation.

\section{Approach to the assessment}

The Working Group III contribution to the AR5 explores the solution space of climate change mitigation drawing on experience and expectations for the future. This exploration is based on a comprehensive and transparent assessment of the scientific, technical, and socio-economic literature on the mitigation of climate change.

The intent of the report is to facilitate an integrated and inclusive deliberation of alternative climate policy goals and the different possible means to achieve them (e.g., technologies, policies, institutional settings). It does so through informing the policymakers and general public about the practical implications of alternative policy options, i. e., their associated costs and benefits, risks and trade-offs.

During the AR5 cycle, the role of the Working Group III scientists was akin to that of a cartographer: they mapped out different pathways within the solution space and assessed potential practical consequences and trade-offs; at the same time, they clearly marked implicit value assumptions and uncertainties. Consequently, this report may now be used by policymakers like a map for navigating the widely unknown territory of climate policy. Instead of providing recommendations for how to solve the complex policy problems, the report offers relevant information that enables policymakers to assess alternative mitigation options.

There are four major pillars to this cartography exercise:

Exploration of alternative climate policy goals: The report lays out the technological, economic and institutional requirements for stabilizing global mean temperature increases at different levels. It informs decision makers about the costs and benefits, risks and opportunities of these, acknowledging the fact that often more than one path can lead to a given policy goal.

Transparency over value judgments: The decision which mitigation path to take is influenced by a series of sometimes disputed normative choices which relate to the long-term stabilization goal itself, the weighing of other social priorities and the policies for achieving the goal. Facts are often inextricably interlinked with values and there is no purely scientific resolution of value dissent. What an assessment can do to support a rational public debate about value conflicts is to make implicit value judgments and ethical viewpoints as transparent as possible. Moreover, controversial policy goals and related ethical standpoints should be discussed in the context of the required means to reach these goals, in particular their possible consequences and side-effects. The potential for adverse side-effects of mitigation actions therefore requires an iterative assessment approach.

Multiple objectives in the context of sustainable development and equity: A comprehensive exploration of the solution space in the field of climate change mitigation recognizes that mitigation itself will only be one objective among others for decision makers. Decision makers may be interested in pursuing a broader concept of well-being. This broader concept also involves the sharing of limited resources within and across countries as well as across generations. Climate change mitigation is discussed here as a multi-objective problem embedded in a broader sustainable development and equity context.

Risk management: Climate change mitigation can be framed as a risk management exercise. It may provide large opportunities to humankind, but will also be associated with risks and uncertainties. Some of those may be of a fundamental nature and cannot be easily reduced or managed. It is therefore a basic requirement for a scientific assessment to communicate these uncertainties, wherever possible, both in their quantitative and qualitative dimension.

\section{Scope of the report}

During the process of scoping and approving the outline of the Working Group III contribution to the AR5, the IPCC focused on those aspects of the current understanding of the science of climate change mitigation that were judged to be most relevant to policymakers.

Working Group III included an extended framing section to provide full transparency over the concepts and methods used throughout the report, highlighting their underlying value judgments. This includes an improved treatment of risks and risk perception, uncertainties, ethical questions as well as sustainable development.

The exploration of the solution space for climate change mitigation starts from a new set of baseline and mitigation scenarios. The entire scenario set for the first time provides fully consistent information on radiative forcing and temperature in broad agreement with the information provided in the Working Group I contribution to the AR5. The United Nations Framework Convention on Climate Change requested the IPCC to provide relevant scientific evidence for reviewing the $2^{\circ} \mathrm{C}$ 
goal as well as a potential $1.5{ }^{\circ} \mathrm{C}$ goal. Compared to the AR4 the report therefore assesses a large number of low stabilization scenarios broadly consistent with the $2^{\circ} \mathrm{C}$ goal. It includes policy scenarios that investigate the impacts of delayed and fragmented international mitigation efforts and of restricted mitigation technologies portfolios on achieving specific mitigation goals and associated costs.

The WGIII contribution to the AR5 features several new elements. A full chapter is devoted to human settlements and infrastructures. Governance structures for the design of mitigation policies are discussed on the global, regional, national and sub-national level. The report closes with a novel chapter about investment needs and finance.

\section{Structure of the report}

The Working Group III contribution to the Fifth Assessment report is comprised of four parts:

\section{Part I: Introduction (Chapter 1)}

Part II: Framing Issues (Chapters 2-4)

Part III: Pathways for Mitigating Climate Change (Chapters 5-12)

Part IV: Assessment of Policies, Institutions and Finance (Chapters 13-16)

Part I provides an introduction to the Working Group III contribution and sets the stage for the subsequent chapters. It describes the 'Lessons learned since AR4' and the 'New challenges for AR5'. It gives a brief overview of 'Historical, current and future trends' regarding GHG emissions and discusses the issues involved in climate change response policies including the ultimate objective of the UNFCCC (Article 2) and the human dimensions of climate change (including sustainable development).

Part II deals with framing issues that provide transparency over methodological foundations and underlying concepts including the relevant value judgments for the detailed assessment of climate change mitigation policies and measures in the subsequent parts. Each chapter addresses key overarching issues (Chapter 2: Integrated Risk and Uncertainty Assessment of Climate Change Response Policies; Chapter 3: Social, Economic and Ethical Concepts and Methods; Chapter 4: Sustainable Development and Equity) and acts as a reference point for subsequent chapters.

Part III provides an integrated assessment of possible mitigation pathways and the respective sectoral contributions and implications. It combines cross-sectoral and sectoral information on long-term mitigation pathways and short- to mid-term mitigation options in major economic sectors. Chapter 5 (Drivers, Trends and Mitigation) provides the context for the subsequent chapters by outlining global trends in stocks and flows of greenhouse gases (GHGs) and short-lived climate pollutants by means of different accounting methods that provide complementary perspectives on the past. It also discusses emissions drivers, which informs the assessment of how GHG emissions have historically developed. Chapter 6 (Assessing Transformation Pathways) analyses 1200 new scenarios generated by 31 modelling teams around the world to explore the economic, technological and institutional prerequisites and implications of mitigation pathways with different levels of ambition. The sectoral chapters (Chapter 7-11) and Chapter 12 (Human Settlements, Infrastructure and Spatial Planning) provide information on the different mitigation options across energy systems, transport, buildings, industry, agriculture, forestry and other land use as well as options specific to human settlements and infrastructure, including the possible co-benefits, adverse side-effects and costs that may be associated with each of these options. Pathways described in Chapter 6 are discussed in a sector-specific context.

Part IV assesses policies across governance scales. Beginning with international cooperation (Chapter 13), it proceeds to the regional (Chapter 14), national and sub-national levels Chapter 15) before concluding with a chapter that assesses cross-cutting investment and financing issues (Chapter 16). It reviews experience with climate change mitigation policies - both the policies themselves and the interactions among policies across sectors and scales - to provide insights to policymakers on the structure of policies which best fulfill evaluation criteria such as environmental and economic effectiveness, and others.

\section{The assessment process}

This Working Group III contribution to the AR5 represents the combined efforts of hundreds of leading experts in the field of climate change mitigation and has been prepared in accordance with the rules and procedures established by the IPCC. A scoping meeting for the AR5 was held in July 2009 and the outlines for the contributions of the three Working Groups were approved at the $31^{\text {st }}$ Session of the Panel in November 2009. Governments and IPCC observer organizations nominated experts for the author teams. The team of 235 Coordinating Lead Authors and Lead Authors plus 38 Review Editors selected by the Working Group III Bureau, was accepted at the $41^{\text {st }}$ Session of the IPCC Bureau in May 2010. More than 170 Contributing Authors provided draft text and information to the author teams at their request. Drafts prepared by the authors were subject to two rounds of formal review and revision followed by a final round of government comments on the Summary for Policymakers. More than 38,000 written comments were submitted by more than 800 expert reviewers and 37 governments. The Review Editors for each chapter monitored the review process to ensure that all substantive review comments received appropriate consideration. The Summary for Policymakers was approved line-by-line and the underlying chapters were then accepted at the $12^{\text {th }}$ Session of IPCC Working Group III from 7-11 April 2014 in Berlin.

\section{Acknowledgements}

Production of this report was a major effort, in which many people from around the world were involved, with a wide variety of contributions. We wish to thank the generous contributions by the governments and 
institutions involved, which enabled the authors, Review Editors and Government and Expert Reviewers to participate in this process.

Writing this report was only possible thanks to the expertise, hard work and commitment to excellence shown throughout by our Coordinating Lead Authors and Lead Authors, with important assistance by many Contributing Authors and Chapter Science Assistants. We would also like to express our appreciation to the Government and Expert Reviewers, acknowledging their time and energy invested to provide constructive and useful comments to the various drafts. Our Review Editors were also critical in the AR5 process, supporting the author teams with processing the comments and assuring an objective discussion of relevant issues.

We would very much like to thank the governments of the Republic of Korea, New Zealand and Ethiopia as well as the University of Vigo and the Economics for Energy Research Centre in Spain, that, in collaboration with local institutions, hosted the crucial IPCC Lead Author Meetings in Changwon (July 2011), Wellington (March 2012), Vigo (November 2012) and Addis Ababa (July 2013). In addition, we would like to thank the governments of India, Peru, Ghana, the United States and Germany for hosting the Expert Meetings in Calcutta (March 2011), Lima (June 2011), Accra (August 2011), Washington D.C. (August 2012), and Potsdam (October 2013), respectively. Finally, we express our appreciation to the Potsdam Institute for Climate Impact Research (PIK) for welcoming our Coordinating Lead Authors on their campus for a concluding meeting (October 2013).

We are especially grateful for the contribution and support of the German Government, in particular the Bundesministerium für Bildung und Forschung (BMBF), in funding the Working Group III Technical Support Unit (TSU). Coordinating this funding, Gregor Laumann and Sylke Lenz of the Deutsches Zentrum für Luft- und Raumfahrt (DLR) were always ready to dedicate time and energy to the needs of the team. We would also like to express our gratitude to the Bundesministerium für Umwelt, Naturschutz, Bau und Reaktorsicherheit (BMUB) for the good collaboration throughout the AR5 cycle and the excellent organization of the $39^{\text {th }}$ Session of the IPCC - and $12^{\text {th }}$ Session of IPCC WGIII - particularly to Nicole Wilke and Lutz Morgenstern. Our thanks also go to Christiane Textor at Deutsche IPCC Koordinierungsstelle for the good collaboration and her dedicated work. We acknowledge the contribution of the Ministry for Science, Technology and Environment (CITMA) of the Republic of Cuba, the Cuban Institute of Meteorology (INSMET) and the Centre for World Economy Studies (CIEM) for their support as well as the United Nations Economic Commission for Africa (UNECA) and its African Climate Policy Centre (ACPC).

We extend our gratitude to our colleagues in the IPCC leadership. The Executive Committee strengthened and facilitated the scientific and procedural work of all three working groups to complete their contributions: Rajendra K. Pachauri, Vicente Barros, Ismail El Gizouli, Taka Hiraishi, Chris Field, Thelma Krug, Hoesung Lee, Qin Dahe, Thomas Stocker, and Jean-Pascal van Ypersele. For his dedication, leadership and insight, we specially thank IPCC chair Rajendra K. Pachauri.

The Working Group III Bureau - consisting of Antonina Ivanova Boncheva (Mexico), Carlo Carraro (Italy), Suzana Kahn Ribeiro (Brazil), Jim Skea (UK), Francis Yamba (Zambia), and Taha Zatari (Saudi Arabia) — provided continuous and thoughtful advice throughout the AR5 process. We would like to thank Renate Christ, Secretary of the IPCC, and the Secretariat staff Gaetano Leone, Jonathan Lynn, Mary Jean Burer, Sophie Schlingemann, Judith Ewa, Jesbin Baidya, Werani Zabula, Joelle Fernandez, Annie Courtin, Laura Biagioni, Amy Smith and Carlos Martin-Novella, Brenda Abrar-Milani and Nina Peeva, who provided logistical support for government liaison and travel of experts from developing and transitional economy countries. Thanks are due to Francis Hayes who served as the conference officer for the Working Group III Approval Session.

Graphics support by Kay Schröder and his team at Daily-Interactive Digitale Kommunikation is greatly appreciated, as is the copy editing by Stacy Hunt and her team at Confluence Communications, the layout work by Gerd Blumenstein and his team at Da-TeX, the index by Stephen Ingle and his team at WordCo and printing by Matt Lloyd and his team at Cambridge University Press. PIK kindly hosted and housed the TSU offices.

Last but not least, it is a pleasure to acknowledge the tireless work of the staff of the Working Group III Technical Support Unit. Our thanks go to Jan Minx, Ellie Farahani, Susanne Kadner, Kristin Seyboth, Anna Adler, Ina Baum, Steffen Brunner, Patrick Eickemeier, Benjamin Kriemann, Jussi Savolainen, Steffen Schlömer, Christoph von Stechow, and Timm Zwickel, for their professionalism, creativity and dedication to coordinate the report writing and to ensure a final product of high quality. They were assisted by Hamed Beheshti, Siri Chrobog, Thomas Day, Sascha Heller, Ceren Hic, Lisa Israel, Daniel Mahringer, Inga Römer, Geraldine Satre-Buisson, Fee Stehle, and Felix Zoll, whose support and dedication are deeply appreciated.

Sincerely,

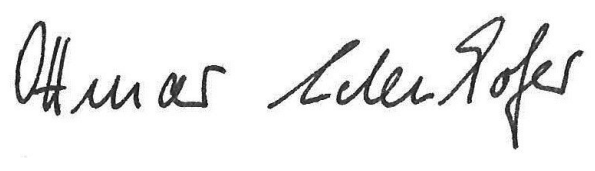

Ottmar Edenhofer

IPCC WG III CO-Chair

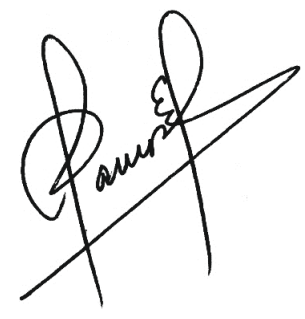

Ramon Pichs-Madruga IPCC WG III CO-Chair

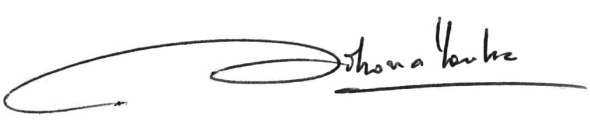

Youba Sokona IPCC WG III CO-Chair 



\section{Dedication}

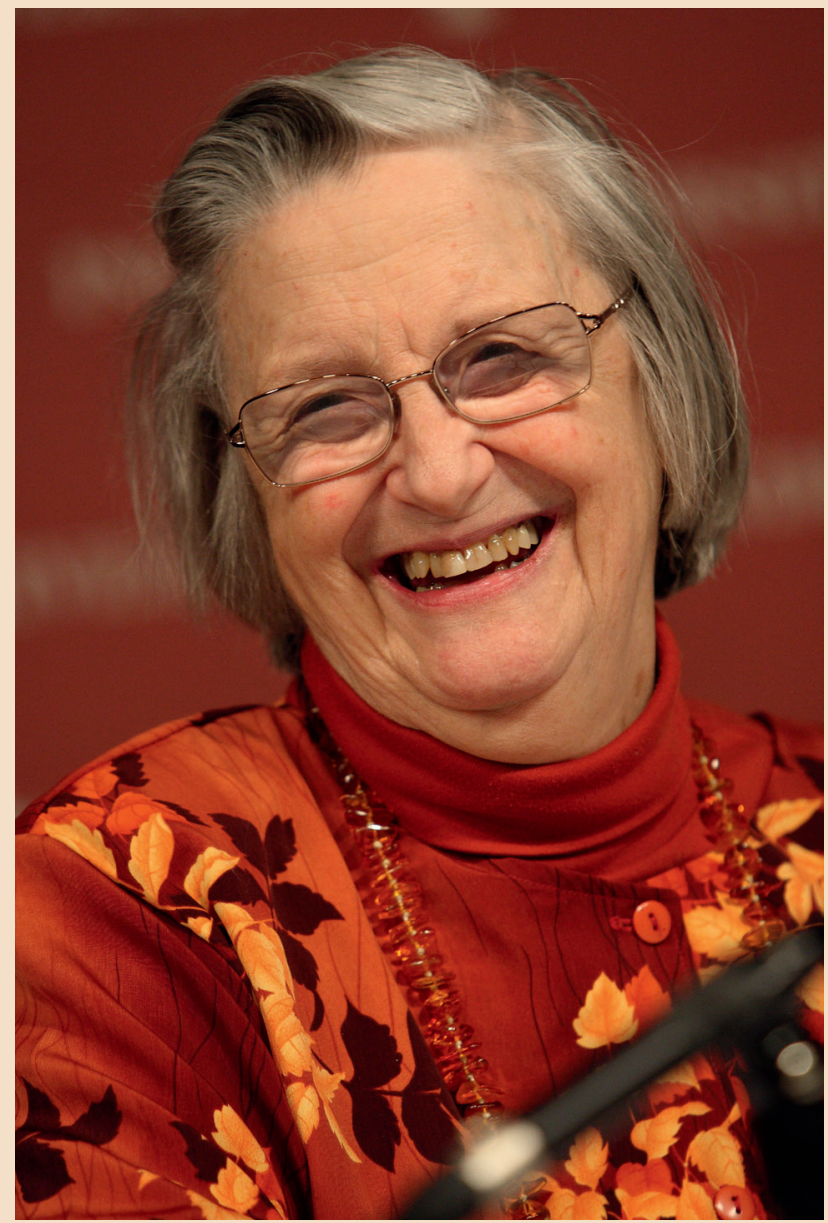

\section{Elinor Ostrom}

(7 August $1933-12$ June 2012)

We dedicate this report to the memory of Elinor Ostrom, Professor of Political Science at Indiana University and Nobel Laureate in Economics. Her work provided a fundamental contribution to the understanding of collective action, trust, and cooperation in the management of common pool resources, including the atmosphere. She launched a research agenda that has encouraged scientists to explore how a variety of overlapping policies at city, national, regional, and international levels can enable humankind to manage the climate problem. The assessment of climate change mitigation across different levels of governance, sectors and regions has been a new focus of the Working Group III contribution to AR5. We have benefited greatly from the vision and intellectual leadership of Elinor Ostrom. 



\title{
In Memoriam
}

\author{
Luxin Huang (1965-2013)
}

Lead Author in Chapter 12 on Human Settlements, Infrastructure and Spatial Planning

Leon Jay (Lee) Schipper (1947-2011)

Review Editor in Chapter 8 on Transport

Luxin Huang contributed to Chapter 12 on Human Settlements, Infrastructure and Spatial Planning. During this time, he was the director of the Department of International Cooperation and Development at the China Academy of Urban Planning and Design (CAUPD) in Beijing, China, where he worked for 27 years. The untimely death of Luxin Huang at the young age of 48 has left the Intergovernmental Panel on Climate Change (IPCC) with great sorrow.

Lee Schipper was a leading scientist in the field of transport, energy and the environment. He was looking forward to his role as review editor for the Transport chapter when he passed away at the age of 64. Schipper had been intimately involved with the IPCC for many years, having contributed as a Lead Author to the IPCC's Second Assessment Report's chapter on Mitigation Options in the Transportation Sector. The IPCC misses his great expertise and guidance, as well as his humorous and musical contributions.

Both researchers were dedicated contributors to the IPCC assessment process. Their passing represents a deep loss for the international scientific community. Luxin Huang and Lee Schipper are dearly remembered by the authors and members of the IPCC Working Group III. 



\section{Contents}

Front Matter

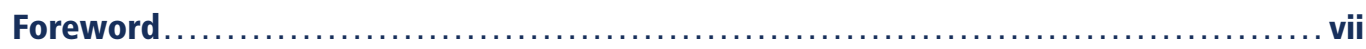

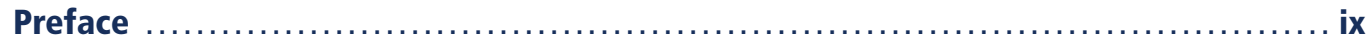

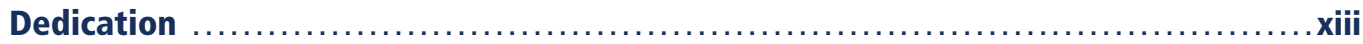

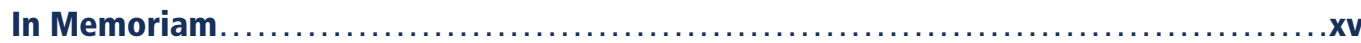

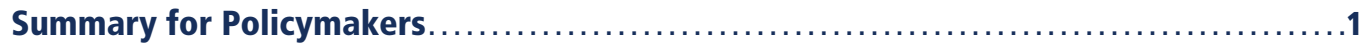

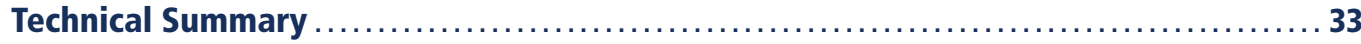

Annex Glossary, Acronyms and Chemical Symbols ............................111 

Summary for Policymakers 



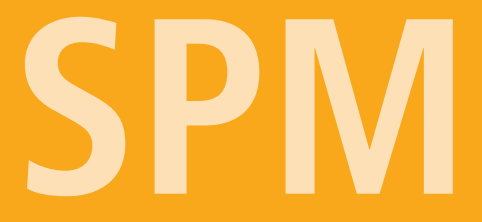

\section{Summary for Policymakers}

\section{Drafting Authors:}

Ottmar Edenhofer (Germany), Ramón Pichs-Madruga (Cuba), Youba Sokona (Mali), Shardul Agrawala (France), Igor Alexeyevich Bashmakov (Russia), Gabriel Blanco (Argentina), John Broome (UK), Thomas Bruckner (Germany), Steffen Brunner (Germany), Mercedes Bustamante (Brazil), Leon Clarke (USA), Felix Creutzig (Germany), Shobhakar Dhakal (Nepal/Thailand), Navroz K. Dubash (India), Patrick Eickemeier (Germany), Ellie Farahani (Canada), Manfred Fischedick (Germany), Marc Fleurbaey (France), Reyer Gerlagh (Netherlands), Luis Gómez-Echeverri (Colombia/Austria), Sujata Gupta (India/Philippines), Jochen Harnisch (Germany), Kejun Jiang (China), Susanne Kadner (Germany), Sivan Kartha (USA), Stephan Klasen (Germany), Charles Kolstad (USA), Volker Krey (Austria/Germany), Howard Kunreuther (USA), Oswaldo Lucon (Brazil), Omar Masera (México), Jan Minx (Germany), Yacob Mulugetta (Ethiopia/UK), Anthony Patt (Austria/Switzerland), Nijavalli H. Ravindranath (India), Keywan Riahi (Austria), Joyashree Roy (India), Roberto Schaeffer (Brazil), Steffen Schlömer (Germany), Karen Seto (USA), Kristin Seyboth (USA), Ralph Sims (New Zealand), Jim Skea (UK), Pete Smith (UK), Eswaran Somanathan (India), Robert Stavins (USA), Christoph von Stechow (Germany), Thomas Sterner (Sweden), Taishi Sugiyama (Japan), Sangwon Suh (Republic of Korea/USA), Kevin Chika Urama (Nigeria/UK/Kenya), Diana Ürge-Vorsatz (Hungary), David G. Victor (USA), Dadi Zhou (China), Ji Zou (China), Timm Zwickel (Germany)

\section{Draft Contributing Authors}

Giovanni Baiocchi (UK/Italy), Helena Chum (Brazil/USA), Jan Fuglestvedt (Norway), Helmut Haberl (Austria), Edgar Hertwich (Austria/Norway), Elmar Kriegler (Germany), Joeri Rogelj (Switzerland/Belgium), H.-Holger Rogner (Germany), Michiel Schaeffer (Netherlands), Steven J. Smith (USA), Detlef van Vuuren (Netherlands), Ryan Wiser (USA)

This Summary for Policymakers should be cited as:

IPCC, 2014: Summary for Policymakers. In: Climate Change 2014: Mitigation of Climate Change. Contribution of Working Group III to the Fifth Assessment Report of the Intergovernmental Panel on Climate Change [Edenhofer, O., R. Pichs-Madruga, Y. Sokona, E. Farahani, S. Kadner, K. Seyboth, A. Adler, I. Baum, S. Brunner, P. Eickemeier, B. Kriemann, J. Savolainen, S. Schlömer, C. von Stechow, T. Zwickel and J.C. Minx (eds.)]. Cambridge University Press, Cambridge, United Kingdom and New York, NY, USA. 



\section{Table of Contents}

SPM.1 Introduction 4

SPM.2 Approaches to climate change mitigation 4

SPM.3 Trends in stocks and flows of greenhouse gases and their drivers 6

SPM.4 Mitigation pathways and measures in the context of sustainable development 10

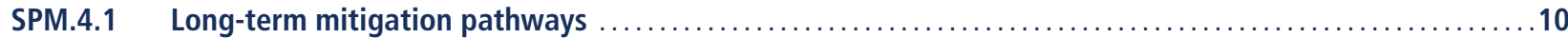

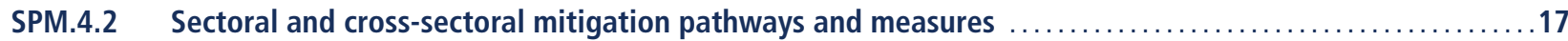

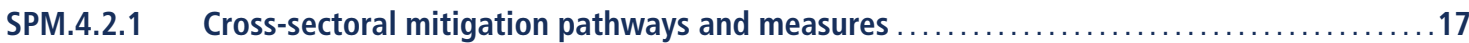

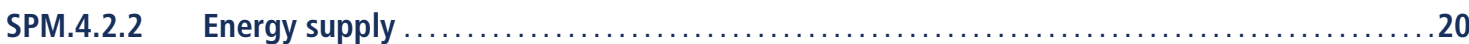

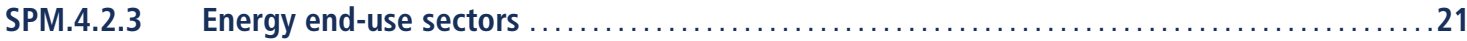

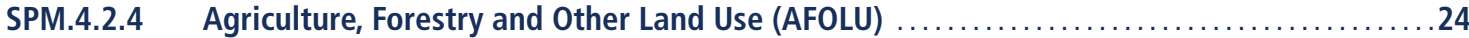

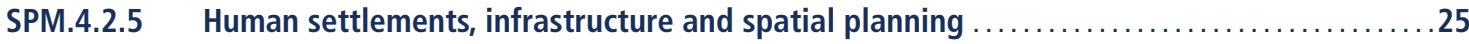

SPM.5 Mitigation policies and institutions …

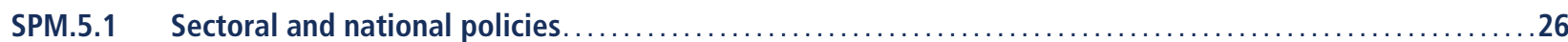

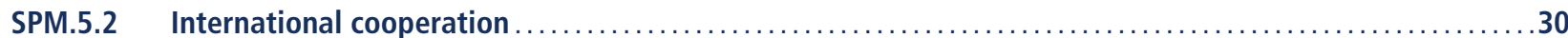




\section{SPM.1 Introduction}

The Working Group III contribution to the IPCC's Fifth Assessment Report (AR5) assesses literature on the scientific, technological, environmental, economic and social aspects of mitigation of climate change. It builds upon the Working Group III contribution to the IPCC's Fourth Assessment Report (AR4), the Special Report on Renewable Energy Sources and Climate Change Mitigation (SRREN) and previous reports and incorporates subsequent new findings and research. The report also assesses mitigation options at different levels of governance and in different economic sectors, and the societal implications of different mitigation policies, but does not recommend any particular option for mitigation.

This Summary for Policymakers (SPM) follows the structure of the Working Group III report. The narrative is supported by a series of highlighted conclusions which, taken together, provide a concise summary. The basis for the SPM can be found in the chapter sections of the underlying report and in the Technical Summary (TS). References to these are given in square brackets.

The degree of certainty in findings in this assessment, as in the reports of all three Working Groups, is based on the author teams' evaluations of underlying scientific understanding and is expressed as a qualitative level of confidence (from very low to very high) and, when possible, probabilistically with a quantified likelihood (from exceptionally unlikely to virtually certain). Confidence in the validity of a finding is based on the type, amount, quality, and consistency of evidence (e. g., data, mechanistic understanding, theory, models, expert judgment) and the degree of agreement. ${ }^{1}$ Probabilistic estimates of quantified measures of uncertainty in a finding are based on statistical analysis of observations or model results, or both, and expert judgment. ${ }^{2}$ Where appropriate, findings are also formulated as statements of fact without using uncertainty qualifiers. Within paragraphs of this summary, the confidence, evidence, and agreement terms given for a bolded finding apply to subsequent statements in the paragraph, unless additional terms are provided.

\section{SPM.2 Approaches to climate change mitigation}

Mitigation is a human intervention to reduce the sources or enhance the sinks of greenhouse gases. Mitigation, together with adaptation to climate change, contributes to the objective expressed in Article 2 of the United Nations Framework Convention on Climate Change (UNFCCC):

The ultimate objective of this Convention and any related legal instruments that the Conference of the Parties may adopt is to achieve, in accordance with the relevant provisions of the Convention, stabilization of greenhouse gas concentrations in the atmosphere at a level that would prevent dangerous anthropogenic interference with the climate system. Such a level should be achieved within a time frame sufficient to allow ecosystems to adapt naturally to climate change, to ensure that food production is not threatened and to enable economic development to proceed in a sustainable manner.

Climate policies can be informed by the findings of science, and systematic methods from other disciplines. [1.2, 2.4, 2.5, Box 3.1]

The following summary terms are used to describe the available evidence: limited, medium, or robust; and for the degree of agreement: low, medium, or high. A level of confidence is expressed using five qualifiers: very low, low, medium, high, and very high, and typeset in italics, e. g., medium confidence. For a given evidence and agreement statement, different confidence levels can be assigned, but increasing levels of evidence and degrees of agreement are correlated with increasing confidence. For more details, please refer to the guidance note for Lead Authors of the IPCC Fifth Assessment Report on consistent treatment of uncertainties.

The following terms have been used to indicate the assessed likelihood of an outcome or a result: virtually certain 99-100\% probability, very likely $90-100 \%$, likely $66-100 \%$, about as likely as not 33-66\%, unlikely $0-33 \%$, very unlikely $0-10 \%$, exceptionally unlikely $0-1 \%$. Additional terms (more likely than not $>50-100 \%$, and more unlikely than likely $0-<50 \%$ ) may also be used when appropriate. Assessed likelihood is typeset in italics, e. g., very likely. 
Sustainable development and equity provide a basis for assessing climate policies and highlight the need for addressing the risks of climate change. ${ }^{3}$ Limiting the effects of climate change is necessary to achieve sustainable development and equity, including poverty eradication. At the same time, some mitigation efforts could undermine action on the right to promote sustainable development, and on the achievement of poverty eradication and equity. Consequently, a comprehensive assessment of climate policies involves going beyond a focus on mitigation and adaptation policies alone to examine development pathways more broadly, along with their determinants. $[4.2,4.3,4.4,4.5,4.6,4.8]$

Effective mitigation will not be achieved if individual agents advance their own interests independently. Climate change has the characteristics of a collective action problem at the global scale, because most greenhouse gases (GHGs) accumulate over time and mix globally, and emissions by any agent (e. g., individual, community, company, country) affect other agents. ${ }^{4}$ International cooperation is therefore required to effectively mitigate GHG emissions and address other climate change issues [1.2.4, 2.6.4, 3.2, 4.2, 13.2, 13.3]. Furthermore, research and development in support of mitigation creates knowledge spillovers. International cooperation can play a constructive role in the development, diffusion and transfer of knowledge and environmentally sound technologies [1.4.4, 3.11.6, 11.8, 13.9, 14.4.3].

Issues of equity, justice, and fairness arise with respect to mitigation and adaptation. ${ }^{5}$ Countries' past and future contributions to the accumulation of GHGs in the atmosphere are different, and countries also face varying challenges and circumstances, and have different capacities to address mitigation and adaptation. The evidence suggests that outcomes seen as equitable can lead to more effective cooperation. [3.10, 4.2.2, 4.6.2]

Many areas of climate policy-making involve value judgements and ethical considerations. These areas range from the question of how much mitigation is needed to prevent dangerous interference with the climate system to choices among specific policies for mitigation or adaptation [3.1, 3.2]. Social, economic and ethical analyses may be used to inform value judgements and may take into account values of various sorts, including human wellbeing, cultural values and non-human values $[3.4,3.10]$.

Among other methods, economic evaluation is commonly used to inform climate policy design. Practical tools for economic assessment include cost-benefit analysis, cost-effectiveness analysis, multi-criteria analysis and expected utility theory [2.5]. The limitations of these tools are well-documented [3.5]. Ethical theories based on social welfare functions imply that distributional weights, which take account of the different value of money to different people, should be applied to monetary measures of benefits and harms [3.6.1, Box TS.2]. Whereas distributional weighting has not frequently been applied for comparing the effects of climate policies on different people at a single time, it is standard practice, in the form of discounting, for comparing the effects at different times [3.6.2].

Climate policy intersects with other societal goals creating the possibility of co-benefits or adverse sideeffects. These intersections, if well-managed, can strengthen the basis for undertaking climate action. Mitigation and adaptation can positively or negatively influence the achievement of other societal goals, such as those related to human health, food security, biodiversity, local environmental quality, energy access, livelihoods, and equitable sustainable development; and vice versa, policies toward other societal goals can influence the achievement of mitigation and adaptation objectives $[4.2,4.3,4.4,4.5,4.6,4.8]$. These influences can be substantial, although sometimes difficult to quantify, especially in welfare terms [3.6.3]. This multi-objective perspective is important in part because it helps to identify areas where support for policies that advance multiple goals will be robust $[1.2 .1,4.2,4.8,6.6 .1]$.

\footnotetext{
See WGII AR5 SPM.

In the social sciences this is referred to as a 'global commons problem'. As this expression is used in the social sciences, it has no specific implications for legal arrangements or for particular criteria regarding effort-sharing.

See FAQ 3.2 for clarification of these concepts. The philosophical literature on justice and other literature can illuminate these issues [3.2, 3.3, 4.6.2].
} 
Climate policy may be informed by a consideration of a diverse array of risks and uncertainties, some of which are difficult to measure, notably events that are of low probability but which would have a significant impact if they occur. Since AR4, the scientific literature has examined risks related to climate change, adaptation, and mitigation strategies. Accurately estimating the benefits of mitigation takes into account the full range of possible impacts of climate change, including those with high consequences but a low probability of occurrence. The benefits of mitigation may otherwise be underestimated (high confidence) [2.5, 2.6, Box 3.9]. The choice of mitigation actions is also influenced by uncertainties in many socio-economic variables, including the rate of economic growth and the evolution of technology (high confidence) $[2.6,6.3]$.

The design of climate policy is influenced by how individuals and organizations perceive risks and uncertainties and take them into account. People often utilize simplified decision rules such as a preference for the status quo. Individuals and organizations differ in their degree of risk aversion and the relative importance placed on near-term versus long-term ramifications of specific actions [2.4]. With the help of formal methods, policy design can be improved by taking into account risks and uncertainties in natural, socio-economic, and technological systems as well as decision processes, perceptions, values and wealth [2.5].

\section{SPM.3 Trends in stocks and flows of greenhouse gases and their drivers}

Total anthropogenic GHG emissions have continued to increase over 1970 to 2010 with larger absolute decadal increases toward the end of this period (high confidence). Despite a growing number of climate change mitigation policies, annual $\mathrm{GHG}$ emissions grew on average by 1.0 gigatonne carbon dioxide equivalent $\left(\mathrm{GtCO}_{2} \mathrm{eq}\right)(2.2 \%)$ per year from 2000 to 2010 compared to $0.4 \mathrm{GtCO}_{2}$ eq (1.3\%) per year from 1970 to 2000 (Figure SPM.1) ${ }^{6,7}{ }^{6}$ Total anthropogenic GHG emissions were the highest in human history from 2000 to 2010 and reached $49( \pm 4.5) \mathrm{GtCO}_{2}$ eq/yr in 2010. The global economic crisis $2007 / 2008$ only temporarily reduced emissions. [1.3, 5.2, 13.3, 15.2.2, Box TS.5, Figure 15.1]

$\mathrm{CO}_{2}$ emissions from fossil fuel combustion and industrial processes contributed about $78 \%$ of the total GHG emission increase from 1970 to 2010, with a similar percentage contribution for the period 2000-2010 (high confidence). Fossil fuel-related $\mathrm{CO}_{2}$ emissions reached $32( \pm 2.7) \mathrm{GtCO}_{2} / \mathrm{yr}$, in 2010 , and grew further by about $3 \%$ between 2010 and 2011 and by about 1-2\% between 2011 and 2012. Of the $49( \pm 4.5) \mathrm{GtCO}_{2}$ eq/yr in total anthropogenic $\mathrm{GHG}$ emissions in $2010, \mathrm{CO}_{2}$ remains the major anthropogenic $\mathrm{GHG}$ accounting for $76 \%(38 \pm 3.8$ $\mathrm{GtCO}_{2}$ eq/yr) of total anthropogenic $\mathrm{GHG}$ emissions in $2010.16 \%\left(7.8 \pm 1.6 \mathrm{GtCO}_{2} \mathrm{eq} / \mathrm{yr}\right)$ come from methane $\left(\mathrm{CH}_{4}\right), 6.2 \%$ $\left(3.1 \pm 1.9 \mathrm{GtCO}_{2} \mathrm{eq} / \mathrm{yr}\right)$ from nitrous oxide $\left(\mathrm{N}_{2} \mathrm{O}\right)$, and $2.0 \%\left(1.0 \pm 0.2 \mathrm{GtCO}_{2} \mathrm{eq} / \mathrm{yr}\right)$ from fluorinated gases (Figure SPM.1). Annually, since 1970 , about $25 \%$ of anthropogenic GHG emissions have been in the form of non- $\mathrm{CO}_{2}$ gases. ${ }^{8}[1.2,5.2]$

\footnotetext{
Throughout the SPM, emissions of GHGs are weighed by Global Warming Potentials with a 100-year time horizon (GWP $\left.{ }_{100}\right)$ from the IPCC Second Assessment Report. All metrics have limitations and uncertainties in assessing consequences of different emissions. [3.9.6, Box TS.5, Annex II.9, WGI SPM]

In this SPM, uncertainty in historic GHG emission data is reported using $90 \%$ uncertainty intervals unless otherwise stated. GHG emission levels are rounded to two significant digits throughout this document; as a consequence, small differences in sums due to rounding may occur.

8 In this report, data on non- $\mathrm{CO}_{2}$ GHGs, including fluorinated gases, are taken from the EDGAR database (Annex II.9), which covers substances included in the Kyoto Protocol in its first commitment period.
} 
Total Annual Anthropogenic GHG Emissions by Groups of Gases 1970-2010

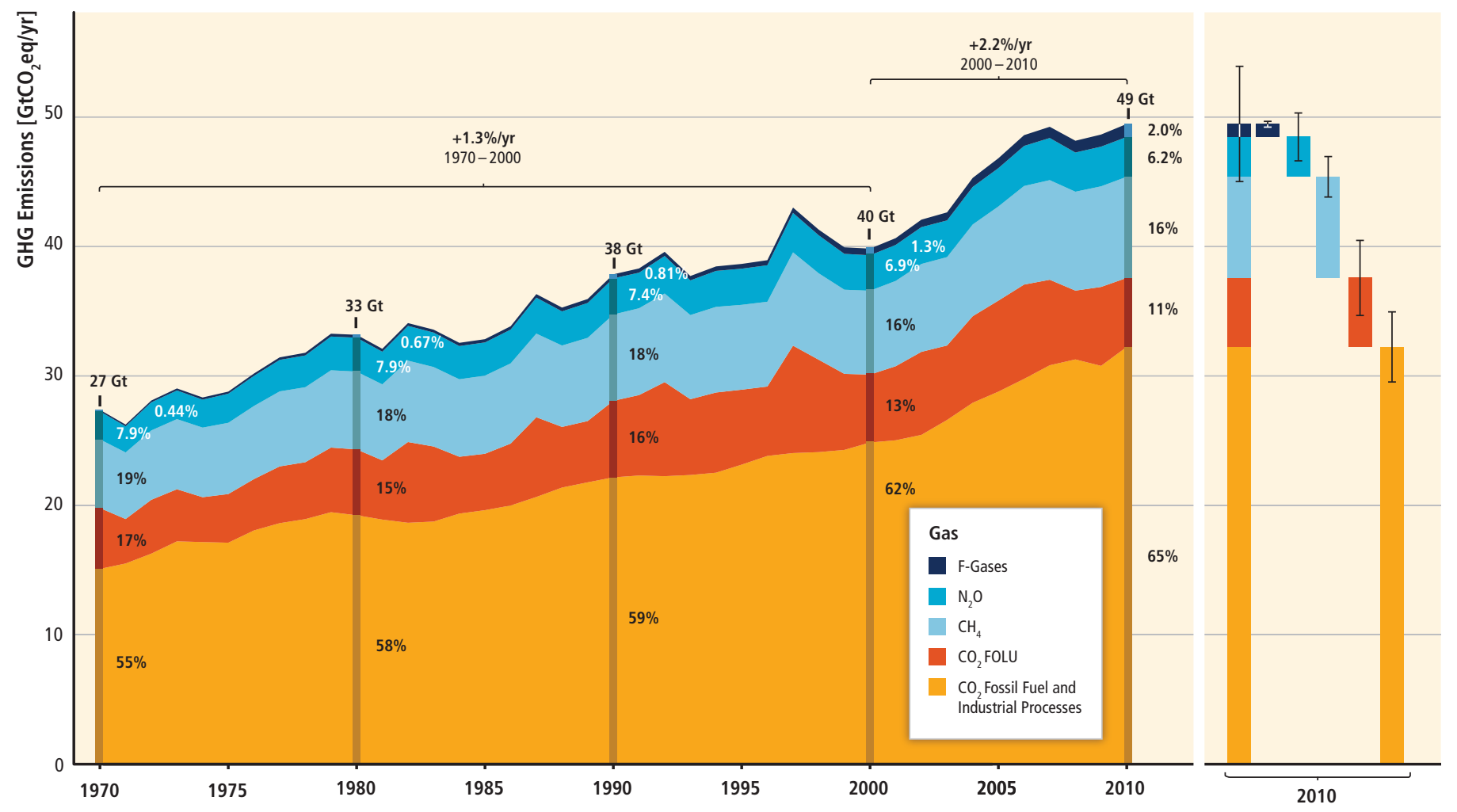

Figure SPM.1 Total annual anthropogenic GHG emissions $\left(\mathrm{GtCO}_{2} \mathrm{eq} / \mathrm{yr}\right)$ by groups of gases 1970-2010: $\mathrm{CO}_{2}$ from fossil fuel combustion and industrial processes; $\mathrm{CO}_{2}$ from Forestry and Other Land Use (FOLU); methane $\left(\mathrm{CH}_{4}\right)$; nitrous oxide $\left(\mathrm{N}_{2} \mathrm{O}\right)$; fluorinated gases ${ }^{8}$ covered under the Kyoto Protocol (F-gases). At the right side of the figure $\mathrm{GHG}$ emissions in 2010 are shown again broken down into these components with the associated uncertainties ( $90 \%$ confidence interval) indicated by the error bars. Total anthropogenic GHG emissions uncertainties are derived from the individual gas estimates as described in Chapter 5 [5.2.3.6]. Global $\mathrm{CO}_{2}$ emissions from fossil fuel combustion are known within $8 \%$ uncertainty ( $90 \%$ confidence interval). $\mathrm{CO}_{2}$ emissions from FOLU have very large uncertainties attached in the order of $\pm 50 \%$. Uncertainty for global emissions of $\mathrm{CH}_{4}, \mathrm{~N}_{2} \mathrm{O}$ and the F-gases has been estimated as 20\%,60\% and $20 \%$, respectively. 2010 was the most recent year for which emission statistics on all gases as well as assessment of uncertainties were essentially complete at the time of data cut-off for this report. Emissions are converted into $\mathrm{CO}_{2}$-equivalents based on GWP ${ }_{100}{ }^{6}$ from the IPCC Second Assessment Report. The emission data from FOLU represents land-based $\mathrm{CO}_{2}$ emissions from forest fires, peat fires and peat decay that approximate to net $\mathrm{CO}_{2}$ flux from FOLU as described in Chapter 11 of this report. Average annual growth rate over different periods is highlighted with the brackets. [Figure 1.3, Figure TS.1]

About half of cumulative anthropogenic $\mathrm{CO}_{2}$ emissions between 1750 and 2010 have occurred in the last 40 years (high confidence). In 1970, cumulative $\mathrm{CO}_{2}$ emissions from fossil fuel combustion, cement production and flaring since 1750 were $420 \pm 35 \mathrm{GtCO}_{2}$; in 2010 , that cumulative total had tripled to $1300 \pm 110 \mathrm{GtCO}_{2}$. Cumulative $\mathrm{CO}_{2}$ emissions from Forestry and Other Land Use (FOLU) ${ }^{9}$ since 1750 increased from $490 \pm 180 \mathrm{GtCO}_{2}$ in 1970 to $680 \pm 300 \mathrm{GtCO}_{2}$ in 2010. [5.2]

Annual anthropogenic GHG emissions have increased by $10 \mathrm{GtCO}_{2}$ eq between 2000 and 2010, with this increase directly coming from energy supply (47\%), industry (30\%), transport (11\%) and buildings (3\%) sectors (medium confidence). Accounting for indirect emissions raises the contributions of the buildings and industry sectors (high confidence). Since $2000, \mathrm{GHG}$ emissions have been growing in all sectors, except AFOLU. Of the $49( \pm 4.5) \mathrm{GtCO}_{2}$ eq emissions in $2010,35 \%\left(17 \mathrm{GtCO}_{2}\right.$ eq) of $\mathrm{GHG}$ emissions were released in the energy supply sector,

9 Forestry and Other Land Use (FOLU) —also referred to as LULUCF (Land Use, Land-Use Change, and Forestry) —is the subset of Agriculture, Forestry and Other Land Use (AFOLU) emissions and removals of GHGs related to direct human-induced land use, land-use change and forestry activities excluding agricultural emissions and removals (see WGIII AR5 Glossary). 
$24 \%$ (12 $\mathrm{GtCO}_{2}$ eq, net emissions) in AFOLU, $21 \%\left(10 \mathrm{GtCO}_{2}\right.$ eq) in industry, $14 \%\left(7.0 \mathrm{GtCO}_{2}\right.$ eq) in transport and $6.4 \%$ ( $3.2 \mathrm{GtCO}_{2} \mathrm{eq}$ ) in buildings. When emissions from electricity and heat production are attributed to the sectors that use the final energy (i.e. indirect emissions), the shares of the industry and buildings sectors in global GHG emissions are increased to $31 \%$ and $19 \%$, respectively (Figure SPM.2). [7.3, 8.2, 9.2, 10.3, 11.2]

Globally, economic and population growth continue to be the most important drivers of increases in $\mathrm{CO}_{2}$ emissions from fossil fuel combustion. The contribution of population growth between 2000 and 2010 remained roughly identical to the previous three decades, while the contribution of economic growth has risen sharply (high confidence). Between 2000 and 2010, both drivers outpaced emission reductions from improvements in energy intensity (Figure SPM.3). Increased use of coal relative to other energy sources has reversed the long-standing trend of gradual decarbonization of the world's energy supply. [1.3, 5.3, 7.2, 14.3, TS.2.2]

Without additional efforts to reduce GHG emissions beyond those in place today, emissions growth is expected to persist driven by growth in global population and economic activities. Baseline scenarios, those without additional mitigation, result in global mean surface temperature increases in 2100 from $3.7^{\circ} \mathrm{C}$ to $4.8^{\circ} \mathrm{C}$ compared to pre-industrial levels ${ }^{10}$ (range based on median climate response; the range is $2.5^{\circ} \mathrm{C}$ to $7.8^{\circ} \mathrm{C}$ when including climate uncertainty, see Table SPM.1 $)^{11}$ (high confidence). The emission scenarios collected for this assessment represent full radiative forcing including GHGs, tropospheric ozone, aerosols and albedo change. Baseline scenarios (scenarios without explicit additional efforts to constrain emissions) exceed 450 parts per million (ppm) $\mathrm{CO}_{2} \mathrm{eq}$ by 2030 and reach $\mathrm{CO}_{2}$ eq concentration levels between 750 and more than 1300 ppm $\mathrm{CO}_{2}$ eq by 2100 . This is similar to the range in atmospheric concentration levels between the RCP 6.0 and RCP 8.5 pathways in $2100 . .^{12}$ For comparison, the $\mathrm{CO}_{2}$ eq concentration in 2011 is estimated to be $430 \mathrm{ppm}$ (uncertainty range $340-520 \mathrm{ppm}$ ). ${ }^{13}$ [6.3, Box TS.6; WGI Figure SPM.5, WGI 8.5, WGI 12.3]

10 Based on the longest global surface temperature dataset available, the observed change between the average of the period $1850-1900$ and of the AR5 reference period $(1986-2005)$ is $0.61^{\circ} \mathrm{C}\left(5-95 \%\right.$ confidence interval: $\left.0.55-0.67^{\circ} \mathrm{C}\right)$ [WGI SPM.E], which is used here as an approximation of the change in global mean surface temperature since pre-industrial times, referred to as the period before 1750.

The climate uncertainty reflects the 5th to 95th percentile of climate model calculations described in Table SPM.1.

12 For the purpose of this assessment, roughly 300 baseline scenarios and 900 mitigation scenarios were collected through an open call from integrated modelling teams around the world. These scenarios are complementary to the Representative Concentration Pathways (RCPs, see WGIII AR5 Glossary). The RCPs are identified by their approximate total radiative forcing in year 2100 relative to 1750: 2.6 Watts per square meter $\left(\mathrm{W} / \mathrm{m}^{2}\right)$ for RCP2.6, 4.5 W/m² for RCP4.5, 6.0 W/m² for RCP6.0, and 8.5 W/m² for RCP8.5. The scenarios collected for this assessment span a slightly broader range of concentrations in the year 2100 than the four RCPs.

13 This is based on the assessment of total anthropogenic radiative forcing for 2011 relative to $1750 \mathrm{in} \mathrm{WGl}$, i.e. $2.3 \mathrm{~W} / \mathrm{m}^{2}$, uncertainty range 1.1 to $3.3 \mathrm{~W} / \mathrm{m}^{2}$. [WGI Figure SPM.5, WGI 8.5, WGI 12.3] 


\section{Greenhouse Gas Emissions by Economic Sectors}

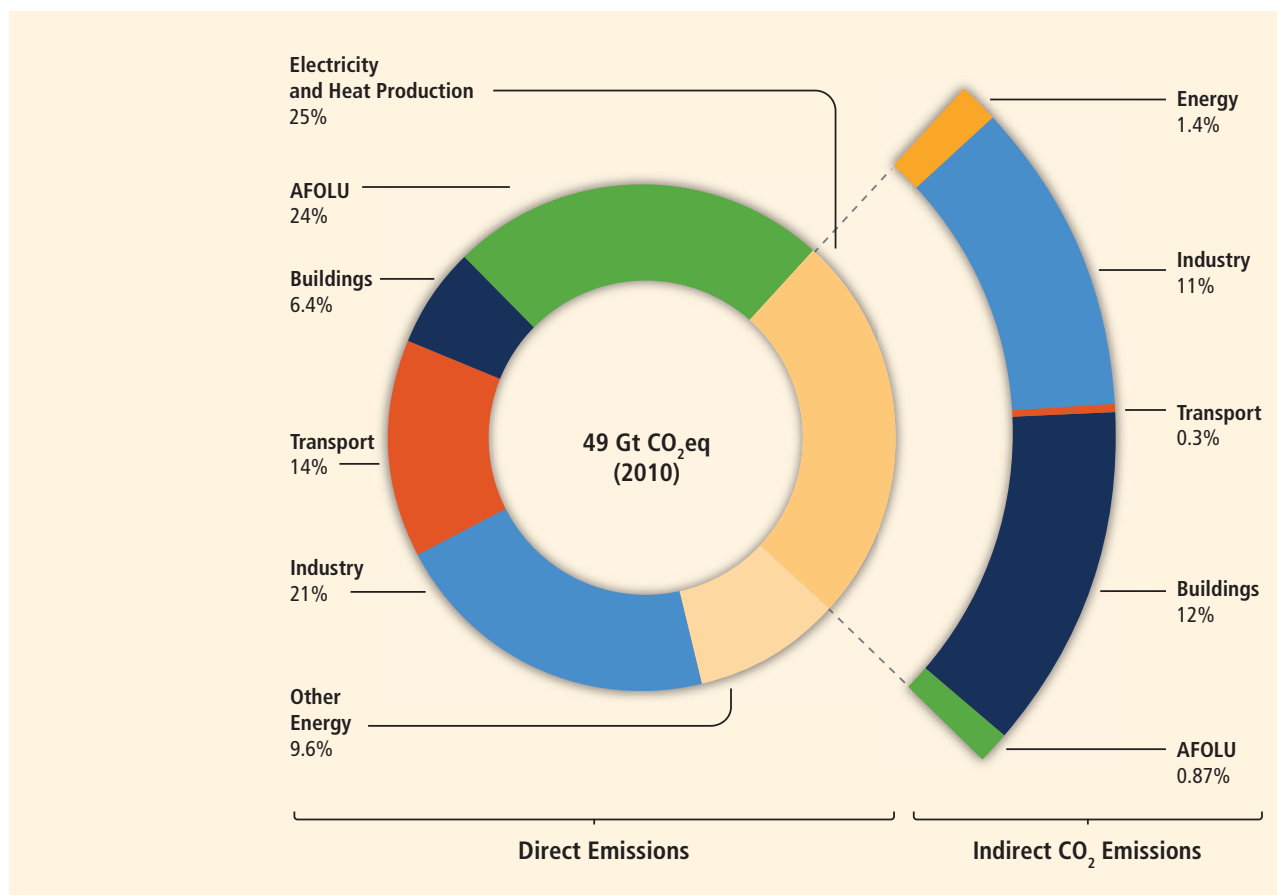

Figure SPM.2 | Total anthropogenic GHG emissions ( $\mathrm{GtCO}_{2} \mathrm{eq} / \mathrm{yr}$ ) by economic sectors. Inner circle shows direct $\mathrm{GHG}$ emission shares (in \% of total anthropogenic $\mathrm{GHG}$ emissions) of five economic sectors in 2010. Pull-out shows how indirect $\mathrm{CO}_{2}$ emission shares (in \% of total anthropogenic GHG emissions) from electricity and heat production are attributed to sectors of final energy use. 'Other Energy' refers to all GHG emission sources in the energy sector as defined in Annex II other than electricity and heat production [A.II.9.1]. The emissions data from Agriculture, Forestry and Other Land Use (AFOLU) includes land-based $\mathrm{CO}_{2}$ emissions from forest fires, peat fires and peat decay that approximate to net $\mathrm{CO}_{2}$ flux from the Forestry and Other Land Use (FOLU) sub-sector as described in Chapter 11 of this report. Emissions are converted into $\mathrm{CO}_{2}$-equivalents based on $\mathrm{GWP}_{100}{ }^{6}$ from the IPCC Second Assessment Report. Sector definitions are provided in Annex II.9. [Figure 1.3a, Figure TS.3 upper panel]

\section{Decomposition of the Change in Total Annual $\mathrm{CO}_{2}$ Emissions from Fossil Fuel Combustion by Decade}

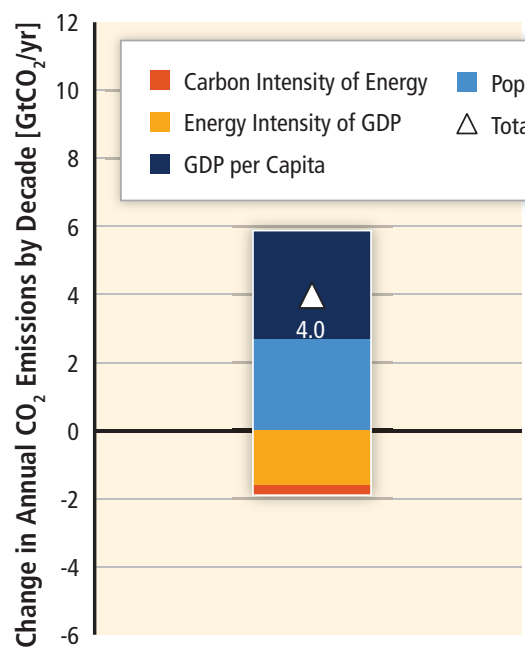

1970-1980

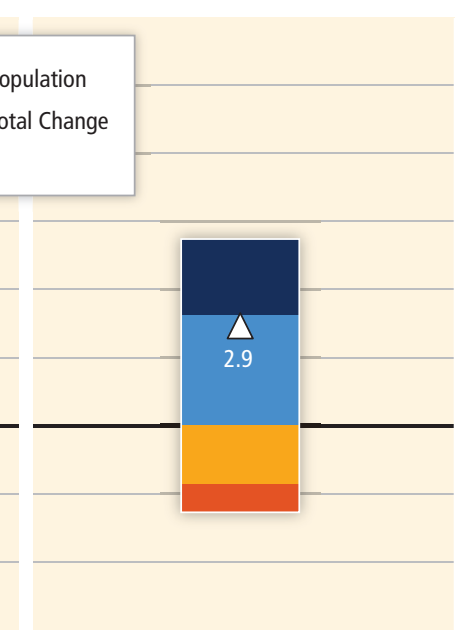

1980-1990

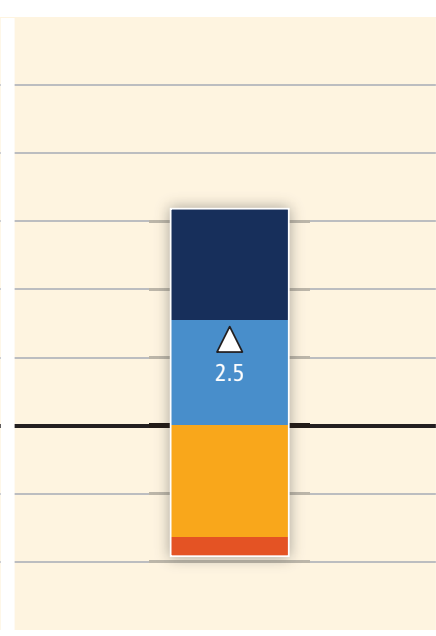

1990-2000

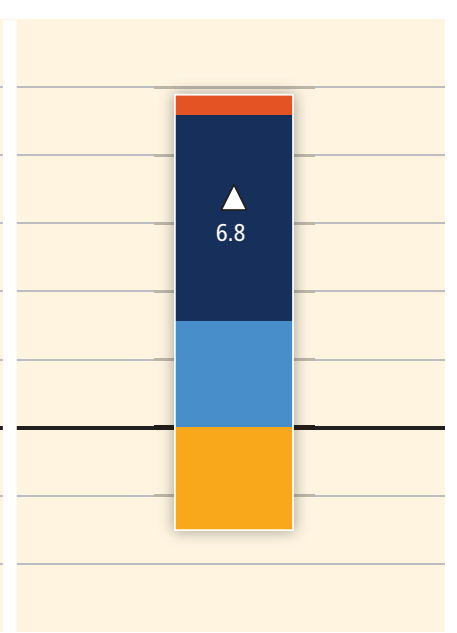

2000-2010

Figure SPM.3 Decomposition of the change in total annual $\mathrm{CO}_{2}$ emissions from fossil fuel combustion by decade and four driving factors: population, income (GDP) per capita, energy intensity of GDP and carbon intensity of energy. The bar segments show the changes associated with each factor alone, holding the respective other factors constant. Total emissions changes are indicated by a triangle. The change in emissions over each decade is measured in gigatonnes of $\mathrm{CO}_{2}$ per year $\left[\mathrm{GtCO}_{2} / \mathrm{yr}\right]$; income is converted into common units using purchasing power parities. [Figure 1.7] 


\section{SPM.4 Mitigation pathways and measures in the context of sustainable development}

SPM.4.1

\section{Long-term mitigation pathways}

There are multiple scenarios with a range of technological and behavioral options, with different characteristics and implications for sustainable development, that are consistent with different levels of mitigation. For this assessment, about 900 mitigation scenarios have been collected in a database based on published integrated models. ${ }^{14}$ This range spans atmospheric concentration levels in 2100 from 430 ppm CO 2 eq to above 720 ppm $\mathrm{CO}_{2}$ eq, which is comparable to the 2100 forcing levels between RCP 2.6 and RCP 6.0. Scenarios outside this range were also assessed including some scenarios with concentrations in 2100 below 430 ppm CO 2 eq (for a discussion of these scenarios see below). The mitigation scenarios involve a wide range of technological, socioeconomic, and institutional trajectories, but uncertainties and model limitations exist and developments outside this range are possible (Figure SPM.4, upper panel).

$[6.1,6.2,6.3$, TS.3.1, Box TS.6]

Mitigation scenarios in which it is likely that the temperature change caused by anthropogenic GHG emissions can be kept to less than $2^{\circ} \mathrm{C}$ relative to pre-industrial levels are characterized by atmospheric concentrations in 2100 of about 450 ppm CO${ }_{2} \mathrm{eq}$ (high confidence). Mitigation scenarios reaching concentration levels of about 500 ppm $\mathrm{CO}_{2}$ eq by 2100 are more likely than not to limit temperature change to less than $2{ }^{\circ} \mathrm{C}$ relative to pre-industrial levels, unless they temporarily 'overshoot' concentration levels of roughly 530 ppm $\mathrm{CO}_{2}$ eq before 2100 , in which case they are about as likely as not to achieve that goal. ${ }^{15}$ Scenarios that reach 530 to 650 ppm $\mathrm{CO}_{2}$ eq concentrations by 2100 are more unlikely than likely to keep temperature change below $2^{\circ} \mathrm{C}$ relative to pre-industrial levels. Scenarios that exceed about $650 \mathrm{ppm} \mathrm{CO}$ eq by 2100 are unlikely to limit temperature change to below $2^{\circ} \mathrm{C}$ relative to pre-industrial levels. Mitigation scenarios in which temperature increase is more likely than not to be less than $1.5^{\circ} \mathrm{C}$ relative to pre-industrial levels by 2100 are characterized by concentrations in 2100 of below 430 ppm $\mathrm{CO}_{2}$ eq. Temperature peaks during the century and then declines in these scenarios. Probability statements regarding other levels of temperature change can be made with reference to Table SPM.1. [6.3, Box TS.6]

Scenarios reaching atmospheric concentration levels of about $450 \mathrm{ppm} \mathrm{CO} \mathrm{C}_{2} \mathrm{eq}$ by 2100 (consistent with a likely chance to keep temperature change below $2{ }^{\circ} \mathrm{C}$ relative to pre-industrial levels) include substantial cuts in anthropogenic GHG emissions by mid-century through large-scale changes in energy systems and potentially land use (high confidence). Scenarios reaching these concentrations by 2100 are characterized by lower global GHG emissions in 2050 than in 2010, $40 \%$ to $70 \%$ lower globally, ${ }^{16}$ and emissions levels near zero $\mathrm{GtCO}_{2}$ eq or below in

\footnotetext{
14 The long-term scenarios assessed in WGIII were generated primarily by large-scale, integrated models that project many key characteristics of mitigation pathways to mid-century and beyond. These models link many important human systems (e. g., energy, agriculture and land use, economy) with physical processes associated with climate change (e.g., the carbon cycle). The models approximate cost-effective solutions that minimize the aggregate economic costs of achieving mitigation outcomes, unless they are specifically constrained to behave otherwise. They are simplified, stylized representations of highly-complex, real-world processes, and the scenarios they produce are based on uncertain projections about key events and drivers over often century-long timescales. Simplifications and differences in assumptions are the reason why output generated from different models, or versions of the same model, can differ, and projections from all models can differ considerably from the reality that unfolds. [Box TS.7, 6.2]

15 Mitigation scenarios, including those reaching 2100 concentrations as high as or higher than about 550 ppm $\mathrm{CO}_{2}$ eq, can temporarily 'overshoot' atmospheric $\mathrm{CO}_{2}$ eq concentration levels before descending to lower levels later. Such concentration overshoot involves less mitigation in the near term with more rapid and deeper emissions reductions in the long run. Overshoot increases the probability of exceeding any given temperature goal. [6.3, Table SPM.1]

16 This range differs from the range provided for a similar concentration category in AR4 ( $50 \%-85 \%$ lower than 2000 for $\mathrm{CO}_{2}$ only). Reasons for this difference include that this report has assessed a substantially larger number of scenarios than in AR4 and looks at all GHGs. In addition, a large proportion of the new scenarios include Carbon Dioxide Removal (CDR) technologies (see below). Other factors include the use of 2100 concentration levels instead of stabilization levels and the shift in reference year from 2000 to 2010. Scenarios with higher emissions in 2050 are characterized by a greater reliance on CDR technologies beyond mid-century.
} 
GHG Emission Pathways 2000-2100: All AR5 Scenarios
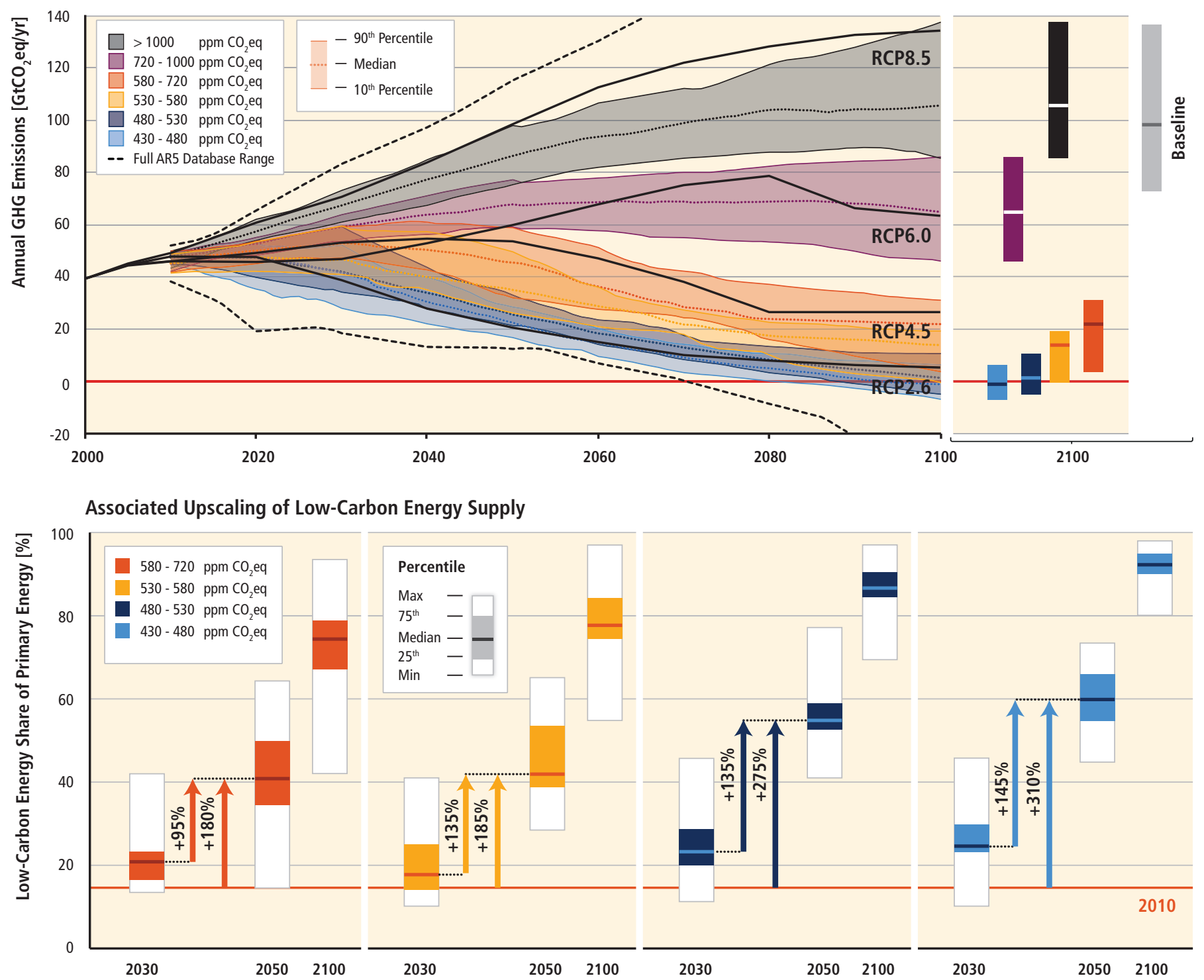

Figure SPM.4| Pathways of global GHG emissions ( $\left.\mathrm{GtCO}_{2} \mathrm{eq} / \mathrm{yr}\right)$ in baseline and mitigation scenarios for different long-term concentration levels (upper panel) [Figure 6.7] and associated upscaling requirements of low-carbon energy (\% of primary energy) for 2030, 2050 and 2100 compared to 2010 levels in mitigation scenarios (lower panel) [Figure 7.16]. The lower panel excludes scenarios with limited technology availability and exogenous carbon price trajectories. For definitions of $\mathrm{CO}_{2}$-equivalent emissions and $\mathrm{CO}_{2}$-equivalent concentrations see the WGIII AR5 Glossary. 
2100. In scenarios reaching about $500 \mathrm{ppm} \mathrm{CO}$ eq by 2100,2050 emissions levels are $25 \%$ to $55 \%$ lower than in 2010 globally. In scenarios reaching about $550 \mathrm{ppm} \mathrm{CO}$ eq, emissions in 2050 are from $5 \%$ above 2010 levels to $45 \%$ below 2010 levels globally (Table SPM.1). At the global level, scenarios reaching about $450 \mathrm{ppm} \mathrm{CO}_{2}$ eq are also characterized by more rapid improvements in energy efficiency and a tripling to nearly a quadrupling of the share of zero- and lowcarbon energy supply from renewables, nuclear energy and fossil energy with carbon dioxide capture and storage (CCS), or bioenergy with CCS (BECCS) by the year 2050 (Figure SPM.4, lower panel). These scenarios describe a wide range of changes in land use, reflecting different assumptions about the scale of bioenergy production, afforestation, and reduced deforestation. All of these emissions, energy, and land-use changes vary across regions. ${ }^{17}$ Scenarios reaching higher concentrations include similar changes, but on a slower timescale. On the other hand, scenarios reaching lower concentrations require these changes on a faster timescale. $[6.3,7.11]$

Mitigation scenarios reaching about $450 \mathrm{ppm} \mathrm{CO}_{2} \mathrm{eq}$ in 2100 typically involve temporary overshoot of

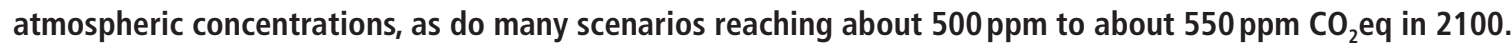
Depending on the level of the overshoot, overshoot scenarios typically rely on the availability and widespread deployment of BECCS and afforestation in the second half of the century. The availability and scale of these and other Carbon Dioxide Removal (CDR) technologies and methods are uncertain and CDR technologies and methods are, to varying degrees, associated with challenges and risks (high confidence) (see Section SPM.4.2).$^{18} \mathrm{CDR}$ is also prevalent in many scenarios without overshoot to compensate for residual emissions from sectors where mitigation is more expensive. There is uncertainty about the potential for large-scale deployment of BECCS, largescale afforestation, and other CDR technologies and methods. [2.6, 6.3, 6.9.1, Figure 6.7, 7.11, 11.13]

Estimated global GHG emissions levels in 2020 based on the Cancún Pledges are not consistent with costeffective long-term mitigation trajectories that are at least about as likely as not to limit temperature change to $2^{\circ} \mathrm{C}$ relative to pre-industrial levels ( 2100 concentrations of about 450 to about $500 \mathrm{ppm} \mathrm{CO}_{2} \mathrm{eq}$ ), but they do not preclude the option to meet that goal (high confidence). Meeting this goal would require further substantial reductions beyond 2020. The Cancún Pledges are broadly consistent with cost-effective scenarios that are likely to keep temperature change below $3^{\circ} \mathrm{C}$ relative to preindustrial levels. [6.4, 13.13, Figure TS.11]

Delaying mitigation efforts beyond those in place today through 2030 is estimated to substantially increase the difficulty of the transition to low longer-term emissions levels and narrow the range of options consistent with maintaining temperature change below $2^{\circ} \mathrm{C}$ relative to pre-industrial levels (high confidence). Costeffective mitigation scenarios that make it at least about as likely as not that temperature change will remain below $2^{\circ} \mathrm{C}$ relative to pre-industrial levels ( 2100 concentrations of about 450 to about $500 \mathrm{ppm} \mathrm{CO}_{2}$ eq) are typically characterized by annual GHG emissions in 2030 of roughly between $30 \mathrm{GtCO}_{2} \mathrm{eq}$ and $50 \mathrm{GtCO}_{2} \mathrm{eq}$ (Figure SPM.5, left panel). Scenarios with annual $\mathrm{GHG}$ emissions above $55 \mathrm{GtCO}_{2}$ eq in 2030 are characterized by substantially higher rates of emissions reductions from 2030 to 2050 (Figure SPM.5, middle panel); much more rapid scale-up of low-carbon energy over this period (Figure SPM.5, right panel); a larger reliance on CDR technologies in the long-term; and higher transitional and long-term economic impacts (Table SPM.2, orange segment). Due to these increased mitigation challenges, many models with annual $2030 \mathrm{GHG}$ emissions higher than $55 \mathrm{GtCO}_{2}$ eq could not produce scenarios reaching atmospheric concentration levels that make it about as likely as not that temperature change will remain below $2{ }^{\circ} \mathrm{C}$ relative to pre-industrial levels. [6.4, 7.11, Figures TS.11, TS.13]

At the national level, change is considered most effective when it reflects country and local visions and approaches to achieving sustainable development according to national circumstances and priorities. [6.4, 11.8.4, WGII SPM]

18 According to WGI, CDR methods have biogeochemical and technological limitations to their potential on the global scale. There is insufficient knowledge to quantify how much $\mathrm{CO}_{2}$ emissions could be partially offset by $\mathrm{CDR}$ on a century timescale. CDR methods carry side-effects and long-term consequences on a global scale. [WGI SPM.E.8] 
Table SPM.1 | Key characteristics of the scenarios collected and assessed for WGIII AR5. For all parameters, the 10th to 90th percentile of the scenarios is shown. ${ }^{1,2}$ [Table 6.3]

\begin{tabular}{|c|c|c|c|c|c|c|c|c|c|c|c|}
\hline \multirow{3}{*}{$\begin{array}{c}\mathrm{CO}_{2} \mathrm{eq} \\
\text { Concentrations } \\
\text { in } 2100[\mathrm{ppm} \\
\left.\mathrm{CO}_{2} \mathrm{eq}\right] \\
\text { Category label } \\
\text { (concentration }^{\text {range) }} \\
\end{array}$} & \multirow{3}{*}{ Subcategories } & \multirow{3}{*}{$\begin{array}{l}\text { Relative } \\
\text { position of } \\
\text { the RCPs }\end{array}$} & \multicolumn{2}{|c|}{$\begin{array}{c}\text { Cumulative } \mathrm{CO}_{2} \\
\text { emissions }{ }^{3}\left[\mathrm{GtCO}_{2}\right] \\
\end{array}$} & \multicolumn{2}{|c|}{$\begin{array}{l}\text { Change in } \mathrm{CO}_{2} \text { eq emissions } \\
\text { compared to } 2010 \text { in }[\%]^{4}\end{array}$} & \multicolumn{5}{|c|}{ Temperature change (relative to $1850-1900)^{5,6}$} \\
\hline & & & \multirow{2}{*}{ 2011-2050 } & \multirow{2}{*}{$2011-2100$} & \multirow{2}{*}{2050} & \multirow{2}{*}{2100} & \multirow{2}{*}{$\begin{array}{c}2100 \\
\text { Temperature } \\
\text { change }\left[{ }^{\circ} \mathrm{C}\right]^{7}\end{array}$} & \multicolumn{4}{|c|}{$\begin{array}{l}\text { Likelihood of staying below temperature } \\
\text { level over the 21st century }\end{array}$} \\
\hline & & & & & & & & $1.5^{\circ} \mathrm{C}$ & $2.0^{\circ} \mathrm{C}$ & $3.0^{\circ} \mathrm{C}$ & $4.0^{\circ} \mathrm{C}$ \\
\hline$<430$ & \multicolumn{11}{|c|}{ Only a limited number of individual model studies have explored levels below $430 \mathrm{ppm} \mathrm{CO}_{2} \mathrm{eq}$} \\
\hline $\begin{array}{c}450 \\
(430-480) \\
\end{array}$ & Total range $\mathrm{e}^{1,10}$ & $\mathrm{RCP} 2.6$ & $550-1300$ & $630-1180$ & -72 to -41 & -118 to -78 & $\begin{array}{c}1.5-1.7 \\
(1.0-2.8)\end{array}$ & $\begin{array}{l}\text { More unlikely } \\
\text { than likely }\end{array}$ & Likely & \multirow{6}{*}{ Likely } & \multirow{8}{*}{ Likely } \\
\hline \multirow{2}{*}{$\begin{array}{c}500 \\
(480-530)\end{array}$} & $\begin{array}{l}\text { No overshoot of } \\
530 \text { ppm } \mathrm{CO}_{2} \text { eq }\end{array}$ & & $860-1180$ & $960-1430$ & -57 to -42 & -107 to -73 & $\begin{array}{c}1.7-1.9 \\
(1.2-2.9)\end{array}$ & \multirow{6}{*}{ Unlikely } & $\begin{array}{c}\text { More likely } \\
\text { than not }\end{array}$ & & \\
\hline & $\begin{array}{c}\text { Overshoot of } \\
530 \mathrm{ppm} \mathrm{CO}_{2} \mathrm{eq} \\
\end{array}$ & & $1130-1530$ & $990-1550$ & -55 to -25 & -114 to -90 & $\begin{array}{r}1.8-2.0 \\
(1.2-3.3) \\
\end{array}$ & & $\begin{array}{c}\text { About as } \\
\text { likely as not }\end{array}$ & & \\
\hline 550 & $\begin{array}{l}\text { No overshoot of } \\
580 \mathrm{ppm} \mathrm{CO}_{2} \mathrm{eq}\end{array}$ & & $1070-1460$ & $1240-2240$ & -47 to -19 & -81 to -59 & $\begin{array}{c}2.0-2.2 \\
(1.4-3.6) \\
\end{array}$ & & \multirow{3}{*}{$\begin{array}{l}\text { More unlikely } \\
\text { than likely }\end{array}$} & & \\
\hline$(530-580)$ & $\begin{array}{l}\text { Overshoot of } \\
580 \mathrm{ppm} \mathrm{CO}_{2} \mathrm{eq}\end{array}$ & & $1420-1750$ & $1170-2100$ & -16 to 7 & -183 to -86 & $\begin{array}{c}2.1-2.3 \\
(1.4-3.6) \\
\end{array}$ & & & & \\
\hline$(580-650)$ & Total range & \multirow{2}{*}{$\mathrm{RCP} 4.5$} & $1260-1640$ & $1870-2440$ & -38 to 24 & -134 to -50 & $\begin{array}{c}2.3-2.6 \\
(1.5-4.2) \\
\end{array}$ & & & & \\
\hline$(650-720)$ & Total range & & $1310-1750$ & $2570-3340$ & -11 to 17 & -54 to -21 & $\begin{array}{r}2.6-2.9 \\
(1.8-4.5) \\
\end{array}$ & & \multirow{2}{*}{ Unlikely } & $\begin{array}{c}\text { More likely } \\
\text { than not }\end{array}$ & \\
\hline$(720-1000)$ & Total range & RCP6.0 & $1570-1940$ & $3620-4990$ & 18 to 54 & -7 to 72 & $\begin{array}{c}3.1-3.7 \\
(2.1-5.8) \\
\end{array}$ & \multirow{2}{*}{ Unlikely" } & & $\begin{array}{l}\text { More unlikely } \\
\text { than likely }\end{array}$ & \\
\hline$>1000$ & Total range & RCP8.5 & $1840-2310$ & $5350-7010$ & 52 to 95 & 74 to 178 & $\begin{array}{c}4.1-4.8 \\
(2.8-7.8)\end{array}$ & & Unlikely"1 & Unlikely & $\begin{array}{l}\text { More unlikely } \\
\text { than likely }\end{array}$ \\
\hline
\end{tabular}

The 'total range' for the 430-480 ppm CO $\mathrm{CO}_{2}$ eq scenarios corresponds to the range of the 10th-90th percentile of the subcategory of these scenarios shown in Table 6.3. Baseline scenarios (see SPM.3) fall into the $>1000$ and 720-1000 ppm CO $\mathrm{CO}_{2}$ eq categories. The latter category also includes mitigation scenarios. The baseline scenarios in the latter category reach a temperature change of $2.5-5.8^{\circ} \mathrm{C}$ above preindustrial in 2100 . Together with the baseline scenarios in the $>1000$ ppm $\mathrm{CO}_{2}$ eq category, this leads to an overall 2100 temperature range of $2.5-7.8^{\circ} \mathrm{C}$ (range based on median climate response: $3.7-4.8^{\circ} \mathrm{C}$ ) for baseline scenarios across both concentration categories.

3 For comparison of the cumulative $\mathrm{CO}_{2}$ emissions estimates assessed here with those presented in WGI, an amount of 515 [445-585] GtC (1890 [1630-2150] GtCO $)$, was already emitted by 2011 since 1870 [Section WGI 12.5]. Note that cumulative emissions are presented here for different periods of time (2011-2050 and 2011-2100) while cumulative emissions in WGI are presented as total compatible emissions for the RCPs (2012-2100) or for total compatible emissions for remaining below a given temperature target with a given likelihood [WGI Table SPM.3, WGI SPM.E.8].

4 The global 2010 emissions are $31 \%$ above the 1990 emissions (consistent with the historic GHG emission estimates presented in this report). $\mathrm{CO}_{2}$ eq emissions include the basket of Kyoto gases $\left(\mathrm{CO}_{2}, \mathrm{CH}_{4}, \mathrm{~N}_{2} \mathrm{O}\right.$ as well as F-gases).

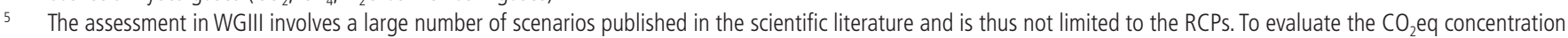
and climate implications of these scenarios, the MAGICC model was used in a probabilistic mode (see Annex II). For a comparison between MAGICC model results and the outcomes of the models used in WGI, see Sections WGI 12.4.1.2 and WGI 12.4.8 and 6.3.2.6. Reasons for differences with WGI SPM Table.2 include the difference in reference year (1986-2005 vs. 1850-1900 here), difference in reporting year (2081-2100 vs 2100 here), set-up of simulation (CMIP5 concentration driven versus MAGICC emission-driven here), and the wider set of scenarios (RCPs versus the full set of scenarios in the WGIII AR5 scenario database here).

6 Temperature change is reported for the year 2100, which is not directly comparable to the equilibrium warming reported in WGIII AR4 [Table 3.5, Chapter 3]. For the 2100 temperature estimates, the transient climate response (TCR) is the most relevant system property. The assumed $90 \%$ range of the TCR for MAGICC is $1.2-2.6^{\circ} \mathrm{C}$ (median $1.8^{\circ} \mathrm{C}$ ). This compares to the $90 \%$ range of TCR between $1.2-2.4^{\circ} \mathrm{C}$ for CMIP5 [WGI 9.7] and an assessed likely range of $1-2.5^{\circ} \mathrm{C}$ from multiple lines of evidence reported in the WGI AR5 [Box 12.2 in Section 12.5].

7 Temperature change in 2100 is provided for a median estimate of the MAGICC calculations, which illustrates differences between the emissions pathways of the scenarios in each category. The range of temperature change in the parentheses includes in addition the carbon cycle and climate system uncertainties as represented by the MAGICC model [see 6.3.2.6 for further details]. The temperature data compared to the 1850-1900 reference year was calculated by taking all projected warming relative to 1986-2005, and adding $0.61^{\circ} \mathrm{C}$ for 1986-2005 compared to 1850-1900, based on HadCRUT4 [see WGI Table SPM.2].

8 The assessment in this table is based on the probabilities calculated for the full ensemble of scenarios in WGIII using MAGICC and the assessment in WGI of the uncertainty of the temperature projections not covered by climate models. The statements are therefore consistent with the statements in WGI, which are based on the CMIP5 runs of the RCPs and the assessed uncertainties. Hence, the likelihood statements reflect different lines of evidence from both WGs. This WGI method was also applied for scenarios with intermediate concentration levels where no CMIP5 runs are available. The likelihood statements are indicative only [6.3], and follow broadly the terms used by the WGI SPM for temperature projections: likely 66-100\%, more likely than not $>50-100 \%$, about as likely as not 33-66\%, and unlikely 0-33\%. In addition the term more unlikely than likely $0-<50 \%$ is used.

9 The $\mathrm{CO}_{2}$-equivalent concentration includes the forcing of all GHGs including halogenated gases and tropospheric ozone, as well as aerosols and albedo change (calculated on the basis of the total forcing from a simple carbon cycle/climate model, MAGICC).

10 The vast majority of scenarios in this category overshoot the category boundary of $480 \mathrm{ppm} \mathrm{CO}_{2}$ eq concentrations.

11 For scenarios in this category no CMIP5 run [WGI Chapter 12, Table 12.3] as well as no MAGICC realization [6.3] stays below the respective temperature level. Still, an unlikely assignment is given to reflect uncertainties that might not be reflected by the current climate models.

12 Scenarios in the 580-650 ppm CO $\mathrm{CO}_{2}$ category include both overshoot scenarios and scenarios that do not exceed the concentration level at the high end of the category (like RCP4.5). The latter type of scenarios, in general, have an assessed probability of more unlikely than likely to stay below the $2^{\circ} \mathrm{C}$ temperature level, while the former are mostly assessed to have an unlikely probability of staying below this level. 
GHG Emissions Pathways to 2030

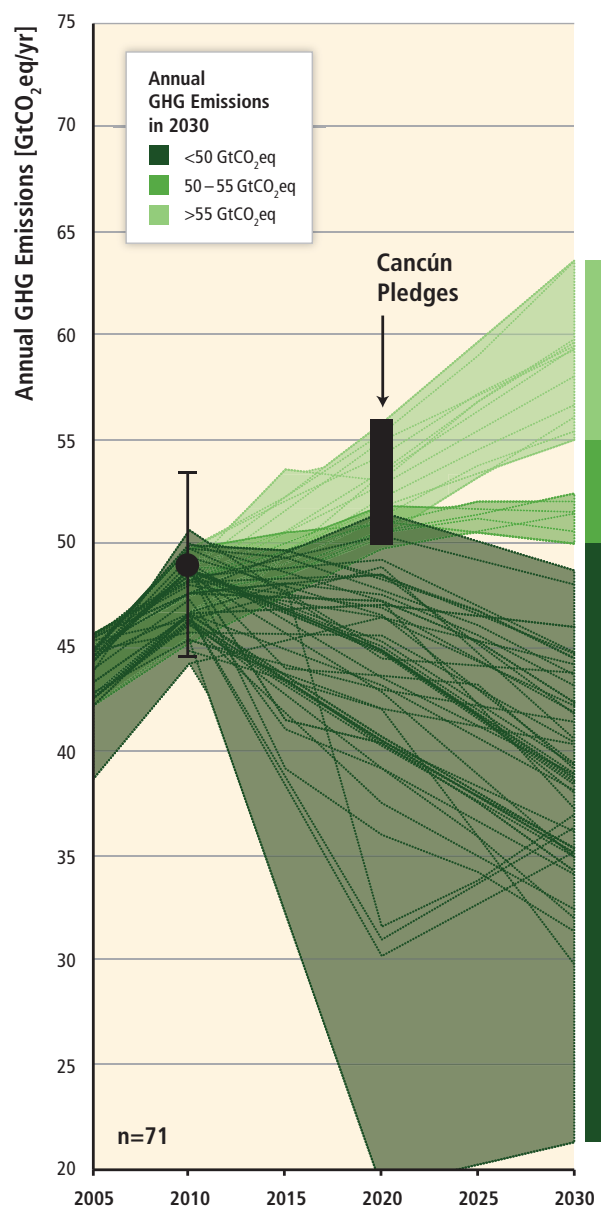

Implications of Different 2030 GHG Emissions

Levels for the Rate of Annual Average $\mathrm{CO}_{2}$ Emissions Reductions from 2030 to 2050
Implications of Different 2030 GHG Emissions Levels for Low-Carbon Energy Upscaling
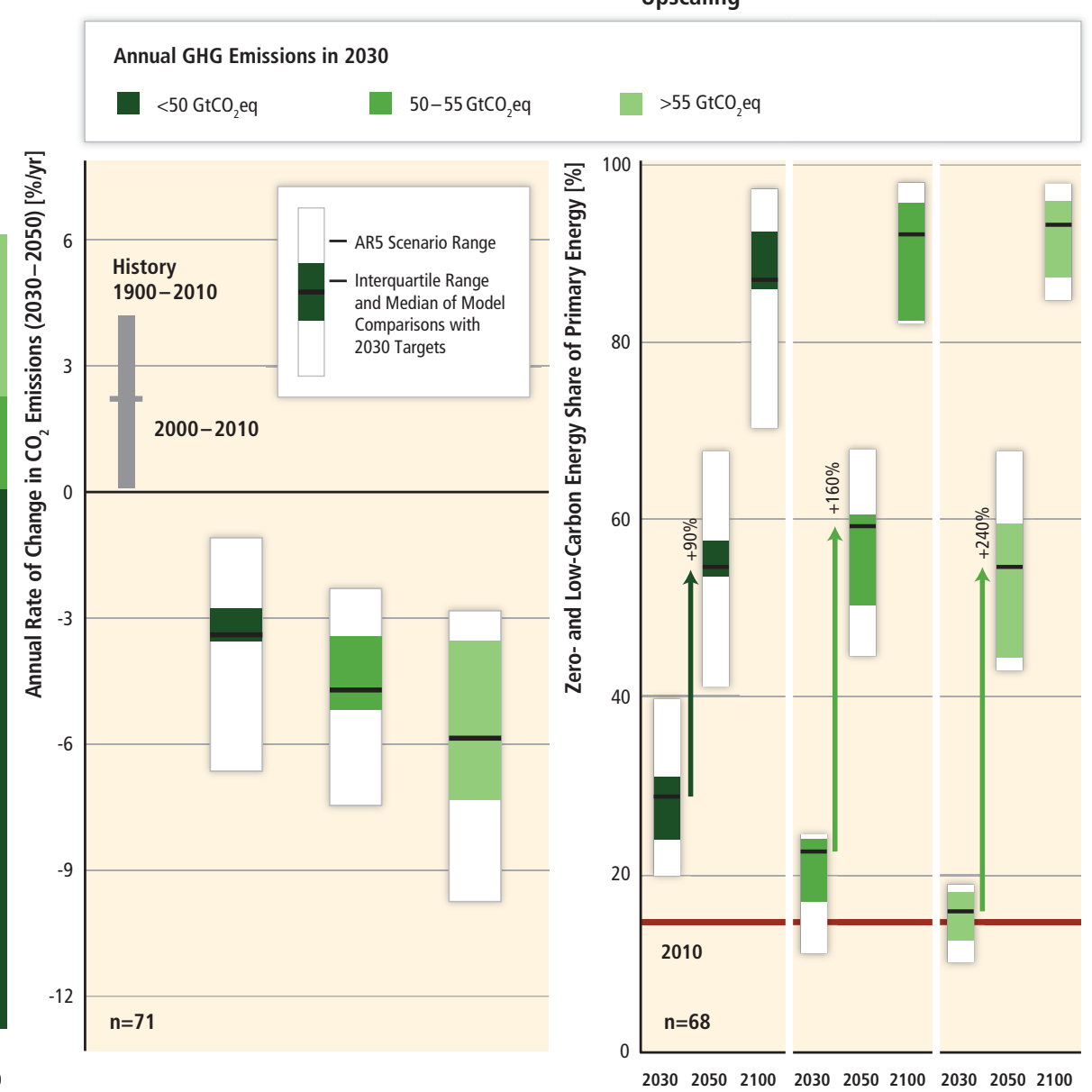

Figure SPM.5 | The implications of different 2030 GHG emissions levels (left panel) for the rate of $\mathrm{CO}_{2}$ emissions reductions from 2030 to 2050 (middle panel) and low-carbon energy upscaling from 2030 to 2050 and 2100 (right panel) in mitigation scenarios reaching about 450 to about $500(430-530)$ ppm $\mathrm{CO}_{2}$ eq concentrations by 2100 . The scenarios are grouped according to different emissions levels by 2030 (coloured in different shades of green). The left panel shows the pathways of $\mathrm{GHG}_{\mathrm{H}} \mathrm{emissions}$ (GtCO $\mathrm{eq}_{2} / \mathrm{yr}$ ) leading to these 2030 levels. The black bar shows the estimated uncertainty range of $\mathrm{GHG}$ emissions implied by the Cancún Pledges. The middle panel denotes the average annual $\mathrm{CO}_{2}$ emissions reduction rates for the period 2030-2050. It compares the median and interquartile range across scenarios from recent intermodel comparisons with explicit 2030 interim goals to the range of scenarios in the Scenario Database for WGIII AR5. Annual rates of historical emissions change between $1900-2010$ (sustained over a period of 20 years) and average annual emissions change between 2000-2010 are shown in grey. The arrows in the right panel show the magnitude of zero and low-carbon energy supply up-scaling from 2030 to 2050 subject to different 2030 GHG emissions levels. Zero- and low-carbon energy supply includes renewables, nuclear energy, fossil energy with carbon dioxide capture and storage (CCS), and bioenergy with CCS (BECCS). Note: Only scenarios that apply the full, unconstrained mitigation technology portfolio of the underlying models (default technology assumption) are shown. Scenarios with large net negative global emissions (>20 $\mathrm{GtCO}_{2} / \mathrm{yr}$ ), scenarios with exogenous carbon price assumptions, and scenarios with 2010 emissions significantly outside the historical range are excluded. The right-hand panel includes only 68 scenarios, because three of the 71 scenarios shown in the figure do not report some subcategories for primary energy that are required to calculate the share of zero- and low-carbon energy. [Figures 6.32 and 7.16; 13.13.1.3] 
Table SPM.2 | Global mitigation costs in cost-effective scenarios ${ }^{1}$ and estimated cost increases due to assumed limited availability of specific technologies and delayed additional mitigation. Cost estimates shown in this table do not consider the benefits of reduced climate change as well as co-benefits and adverse side-effects of mitigation. The yellow columns show consumption losses in the years 2030,2050, and 2100 and annualized consumption growth reductions over the century in cost-effective scenarios relative to a baseline development without climate policy. The grey columns show the percentage increase in discounted costs ${ }^{2}$ over the century, relative to cost-effective scenarios, in scenarios in which technology is constrained relative to default technology assumptions. ${ }^{3}$ The orange columns show the increase in mitigation costs over the periods 2030-2050 and 2050-2100, relative to scenarios with immediate mitigation, due to delayed additional mitigation through $2030 .{ }^{4}$ These scenarios with delayed additional mitigation are grouped by emission levels of less or more than $55 \mathrm{GtCO}_{2}$ eq in 2030, and two concentration ranges in 2100 (430-530 ppm CO,eq and 530-650 ppm CO eq). In all figures, the median of the scenario set is shown without parentheses, the range between the 16th and 84th percentile of the scenario set is shown in the parentheses, and the number of scenarios in the set is shown in square brackets. ${ }^{5}$ [Figures TS.12, TS.13, 6.21, 6.24, 6.25, Annex II. 10]

\begin{tabular}{|c|c|c|c|c|c|c|c|c|c|c|c|c|}
\hline & \multicolumn{4}{|c|}{ Consumption losses in cost-effective scenarios ${ }^{1}$} & \multicolumn{4}{|c|}{$\begin{array}{l}\text { Increase in total discounted mitigation costs in } \\
\text { scenarios with limited availability of technologies }\end{array}$} & \multicolumn{4}{|c|}{$\begin{array}{l}\text { Increase in medium- and long-term mitigation costs } \\
\text { due to delayed additional mitigation until } 2030\end{array}$} \\
\hline & \multicolumn{3}{|c|}{$\begin{array}{l}\text { [\% reduction in consumption } \\
\text { relative to baseline] }\end{array}$} & \multirow{3}{*}{$\begin{array}{c}\text { [percentage } \\
\text { point } \\
\text { reduction in } \\
\text { annualized } \\
\text { consumption } \\
\text { growth rate] }\end{array}$} & \multicolumn{4}{|c|}{$\begin{array}{l}{[\% \text { increase in total discounted mitigation costs }} \\
(2015-2100) \text { relative to default technology assumptions] }\end{array}$} & \multicolumn{4}{|c|}{$\begin{array}{l}\text { [\% increase in mitigation costs relative } \\
\text { to immediate mitigation] }\end{array}$} \\
\hline & \multirow{2}{*}{2030} & \multirow{2}{*}{2050} & \multirow{2}{*}{2100} & & \multirow{2}{*}{ No CCS } & \multirow{2}{*}{$\begin{array}{l}\text { Nuclear } \\
\text { phase out }\end{array}$} & \multirow{2}{*}{$\begin{array}{l}\text { Limited } \\
\text { Solar/Wind }\end{array}$} & \multirow{2}{*}{$\begin{array}{l}\text { Limited } \\
\text { Bioenergy }\end{array}$} & \multicolumn{2}{|c|}{$\leq 55 \mathrm{GtCO}_{2} \mathrm{eq}$} & \multicolumn{2}{|c|}{$>55 \mathrm{GtCO}_{2} \mathrm{eq}$} \\
\hline $\begin{array}{l}\text { Concentration } \\
{\left[\mathrm{ppm} \mathrm{CO}_{2} \mathrm{eq}\right]}\end{array}$ & & & & & & & & & $2030-2050$ & $2050-2100$ & $2030-2050$ & $2050-2100$ \\
\hline $450(430-480)$ & $\begin{array}{c}1.7 \\
(1.0-3.7) \\
{[N: 14]}\end{array}$ & $\begin{array}{c}3.4 \\
(2.1-6.2)\end{array}$ & $\begin{array}{c}4.8 \\
(2.9-11.4)\end{array}$ & $\begin{array}{c}0.06 \\
(0.04-0.14)\end{array}$ & $\begin{array}{c}138 \\
(29-297) \\
{[N: 4]}\end{array}$ & $\begin{array}{c}7 \\
(4-18) \\
{[\mathrm{N}: 8]} \\
\end{array}$ & $\begin{array}{c}6 \\
(2-29) \\
{[N: 8]}\end{array}$ & $\begin{array}{c}64 \\
(44-78) \\
{[\mathrm{N}: 8]}\end{array}$ & \multirow{2}{*}{$\begin{array}{c}28 \\
(14-50) \\
{[N: 34]}\end{array}$} & \multirow{2}{*}{$\begin{array}{c}15 \\
(5-59)\end{array}$} & \multirow{2}{*}{$\begin{array}{c}44 \\
(2-78) \\
{[\mathrm{N}: 29]}\end{array}$} & \multirow{2}{*}{$\begin{array}{c}37 \\
(16-82)\end{array}$} \\
\hline $500(480-530)$ & $\begin{array}{c}1.7 \\
(0.6-2.1) \\
{[\mathrm{N}: 32]}\end{array}$ & $\begin{array}{c}2.7 \\
(1.5-4.2)\end{array}$ & $\begin{array}{c}4.7 \\
(2.4-10.6)\end{array}$ & $\begin{array}{c}0.06 \\
(0.03-0.13)\end{array}$ & N/A & $\mathrm{N} / \mathrm{A}$ & N/A & N/A & & & & \\
\hline $550(530-580)$ & $\begin{array}{c}0.6 \\
(0.2-1.3) \\
{[\mathrm{N}: 46]}\end{array}$ & $\begin{array}{c}1.7 \\
(1.2-3.3)\end{array}$ & $\begin{array}{c}3.8 \\
(1.2-7.3)\end{array}$ & $\begin{array}{c}0.04 \\
(0.01-0.09)\end{array}$ & $\begin{array}{c}39 \\
(18-78) \\
{[\mathrm{N}: 11]} \\
\end{array}$ & $\begin{array}{c}13 \\
(2-23) \\
{[N: 10]} \\
\end{array}$ & $\begin{array}{c}8 \\
(5-15) \\
{[N: 10]}\end{array}$ & $\begin{array}{c}18 \\
(4-66) \\
{[N: 12]}\end{array}$ & \multirow{2}{*}{$\begin{array}{c}3 \\
(-5-16) \\
{[N: 14]}\end{array}$} & \multirow{2}{*}{$\begin{array}{c}4 \\
(-4-11)\end{array}$} & \multirow{2}{*}{$\begin{array}{c}15 \\
(3-32) \\
{[N: 10]}\end{array}$} & \multirow{2}{*}{$\begin{array}{c}16 \\
(5-24)\end{array}$} \\
\hline $580-650$ & $\begin{array}{c}0.3 \\
(0-0.9) \\
{[\mathrm{N}: 16]}\end{array}$ & $\begin{array}{c}1.3 \\
(0.5-2.0)\end{array}$ & $\begin{array}{c}2.3 \\
(1.2-4.4)\end{array}$ & $\begin{array}{c}0.03 \\
(0.01-0.05)\end{array}$ & $\mathrm{N} / \mathrm{A}$ & $\mathrm{N} / \mathrm{A}$ & $\mathrm{N} / \mathrm{A}$ & N/A & & & & \\
\hline
\end{tabular}

Cost-effective scenarios assume immediate mitigation in all countries and a single global carbon price, and impose no additional limitations on technology relative to the models' default technology assumptions.

2 Percentage increase of net present value of consumption losses in percent of baseline consumption (for scenarios from general equilibrium models) and abatement costs in percent of baseline GDP (for scenarios from partial equilibrium models) for the period 2015-2100, discounted at $5 \%$ per year.

3 No CCS: CCS is not included in these scenarios. Nuclear phase out: No addition of nuclear power plants beyond those under construction, and operation of existing plants until the end of their lifetime. Limited Solar/Wind: a maximum of $20 \%$ global electricity generation from solar and wind power in any year of these scenarios. Limited Bioenergy: a maximum of $100 \mathrm{EJ} / \mathrm{yr}$ modern bioenergy supply globally (modern bioenergy used for heat, power, combinations, and industry was around $18 \mathrm{EJ} / \mathrm{yr}$ in 2008 [11.13.5]). Percentage increase of total undiscounted mitigation costs for the periods 2030-2050 and 2050-2100.

5 The range is determined by the central scenarios encompassing the 16th and 84th percentile of the scenario set. Only scenarios with a time horizon until 2100 are included.

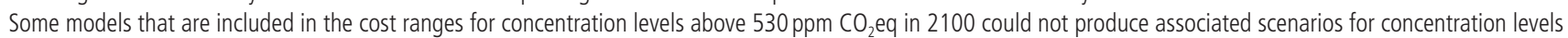
below 530 ppm $\mathrm{CO}_{2}$ eq in 2100 with assumptions about limited availability of technologies and/or delayed additional mitigation.

Estimates of the aggregate economic costs of mitigation vary widely and are highly sensitive to model design and assumptions as well as the specification of scenarios, including the characterization of technologies and the timing of mitigation (high confidence). Scenarios in which all countries of the world begin mitigation immediately, there is a single global carbon price, and all key technologies are available, have been used as a cost-effective benchmark for estimating macroeconomic mitigation costs (Table SPM.2, yellow segments). Under these assumptions, mitigation scenarios that reach atmospheric concentrations of about $450 \mathrm{ppm} \mathrm{CO}$ eq by 2100 entail losses in global consumptionnot including benefits of reduced climate change as well as co-benefits and adverse side-effects of mitigation ${ }^{19}$ - of $1 \%$ to $4 \%$ (median: $1.7 \%$ ) in 2030, $2 \%$ to $6 \%$ (median: $3.4 \%$ ) in 2050, and $3 \%$ to $11 \%$ (median: $4.8 \%$ ) in 2100 relative to consumption in baseline scenarios that grows anywhere from $300 \%$ to more than $900 \%$ over the century. These numbers

19 The total economic effect at different temperature levels would include mitigation costs, co-benefits of mitigation, adverse side-effects of mitigation, adaptation costs and climate damages. Mitigation cost and climate damage estimates at any given temperature level cannot be compared to evaluate the costs and benefits of mitigation. Rather, the consideration of economic costs and benefits of mitigation should include the reduction of climate damages relative to the case of unabated climate change. 
Co-Benefits of Climate Change Mitigation for Air Quality

Impact of Stringent Climate Policy on Air Pollutant Emissions (Global, 2005-2050)

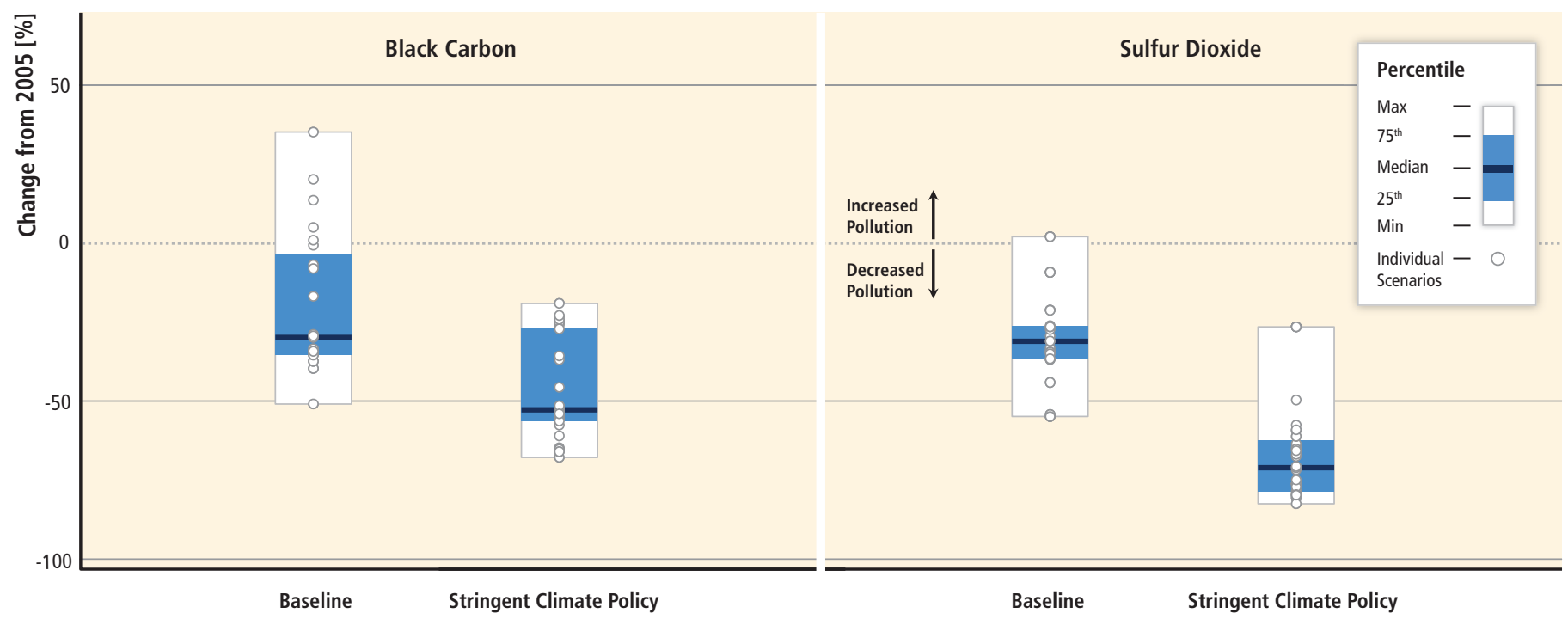

Figure SPM.6 Air pollutant emission levels for black carbon (BC) and sulfur dioxide $\left(\mathrm{SO}_{2}\right)$ in 2050 relative to 2005 ( $0=2005$ levels). Baseline scenarios without additional efforts to reduce GHG emissions beyond those in place today are compared to scenarios with stringent mitigation policies, which are consistent with reaching about 450 to about 500 (430-530) ppm $\mathrm{CO}_{2}$ eq concentrations by 2100. [Figure 6.33]

correspond to an annualized reduction of consumption growth by 0.04 to 0.14 (median: 0.06 ) percentage points over the century relative to annualized consumption growth in the baseline that is between $1.6 \%$ and $3 \%$ per year. Estimates at the high end of these cost ranges are from models that are relatively inflexible to achieve the deep emissions reductions required in the long run to meet these goals and/or include assumptions about market imperfections that would raise costs. Under the absence or limited availability of technologies, mitigation costs can increase substantially depending on the technology considered (Table SPM.2, grey segment). Delaying additional mitigation further increases mitigation costs in the medium- to long-term (Table SPM.2, orange segment). Many models could not achieve atmospheric concentration levels of about $450 \mathrm{ppm} \mathrm{CO}$ eq by 2100 if additional mitigation is considerably delayed or under limited availability of key technologies, such as bioenergy, CCS, and their combination (BECCS). [6.3]

Only a limited number of studies have explored scenarios that are more likely than not to bring temperature change back to below $1.5^{\circ} \mathrm{C}$ by 2100 relative to pre-industrial levels; these scenarios bring atmospheric concentrations to below $430 \mathrm{ppm} \mathrm{CO}_{2}$ eq by 2100 (high confidence). Assessing this goal is currently difficult because no multi-model studies have explored these scenarios. Scenarios associated with the limited number of published studies exploring this goal are characterized by (1) immediate mitigation action; (2) the rapid upscaling of the full portfolio of mitigation technologies; and (3) development along a low-energy demand trajectory. ${ }^{20}[6.3,7.11]$

Mitigation scenarios reaching about 450 to about $500 \mathrm{ppm} \mathrm{CO} \mathrm{CO}_{2}$ eq by 2100 show reduced costs for achieving air quality and energy security objectives, with significant co-benefits for human health, ecosystem impacts, and sufficiency of resources and resilience of the energy system; these scenarios did not quantify other co-benefits or adverse side-effects (medium confidence). These mitigation scenarios show improvements in terms of the sufficiency of resources to meet national energy demand as well as the resilience of energy supply, resulting in energy systems that are less vulnerable to price volatility and supply disruptions. The benefits from reduced impacts to

20 In these scenarios, the cumulative $\mathrm{CO}_{2}$ emissions range between 680 and $800 \mathrm{GtCO}_{2}$ for the period $2011-2050$ and between 90 and $310 \mathrm{GtCO}_{2}$ for the period 2011-2100. Global $\mathrm{CO}_{2}$ eq emissions in 2050 are between 70 and $95 \%$ below 2010 emissions, and they are between 110 and $120 \%$ below 2010 emissions in 2100 . 
health and ecosystems associated with major cuts in air pollutant emissions (Figure SPM.6) are particularly high where currently legislated and planned air pollution controls are weak. There is a wide range of co-benefits and adverse side-effects for additional objectives other than air quality and energy security. Overall, the potential for co-benefits of energy end-use measures outweighs the potential for adverse side-effects, whereas the evidence suggests this may not be the case for all energy supply and AFOLU measures. [WGIII 4.8, 5.7, 6.3.6, 6.6, 7.9, 8.7, 9.7, 10.8, 11.7, 11.13.6, 12.8, Figure TS.14, Table 6.7, Tables TS.3-TS.7; WGII 11.9]

There is a wide range of possible adverse side-effects as well as co-benefits and spillovers from climate policy that have not been well-quantified (high confidence). Whether or not side-effects materialize, and to what extent side-effects materialize, will be case- and site-specific, as they will depend on local circumstances and the scale, scope, and pace of implementation. Important examples include biodiversity conservation, water availability, food security, income distribution, efficiency of the taxation system, labour supply and employment, urban sprawl, and the sustainability of the growth of developing countries. [Box TS.11]

Mitigation efforts and associated costs vary between countries in mitigation scenarios. The distribution of costs across countries can differ from the distribution of the actions themselves (high confidence). In globally cost-effective scenarios, the majority of mitigation efforts takes place in countries with the highest future emissions in baseline scenarios. Some studies exploring particular effort-sharing frameworks, under the assumption of a global carbon market, have estimated substantial global financial flows associated with mitigation for scenarios leading to 2100 atmospheric concentrations of about 450 to about 550 ppm CO 2 eq. [4.6, 6.3.6, 13.4.2.4; Box 3.5; Table 6.4; Figures 6.9, 6.27, $6.28,6.29]$

Mitigation policy could devalue fossil fuel assets and reduce revenues for fossil fuel exporters, but differences between regions and fuels exist (high confidence). Most mitigation scenarios are associated with reduced revenues from coal and oil trade for major exporters (high confidence). The effect of mitigation on natural gas export revenues is more uncertain, with some studies showing possible benefits for export revenues in the medium term until about 2050 (medium confidence). The availability of CCS would reduce the adverse effect of mitigation on the value of fossil fuel assets (medium confidence). [6.3.6, 6.6, 14.4.2]

SPM.4.2

SPM.4.2.1

\section{Sectoral and cross-sectoral mitigation pathways and measures}

\section{Cross-sectoral mitigation pathways and measures}

In baseline scenarios, GHG emissions are projected to grow in all sectors, except for net $\mathrm{CO}_{2}$ emissions in the AFOLU sector ${ }^{21}$ (robust evidence, medium agreement). Energy supply sector emissions are expected to continue to be the major source of GHG emissions, ultimately accounting for the significant increases in indirect emissions from electricity use in the buildings and industry sectors. In baseline scenarios, while non- $\mathrm{CO}_{2} \mathrm{GHG}$ agricultural emissions are projected to increase, net $\mathrm{CO}_{2}$ emissions from the AFOLU sector decline over time, with some models projecting a net sink towards the end of the century (Figure SPM.7). ${ }^{22}[6.3 .1 .4,6.8$, Figure TS.15]

21 Net AFOLU $\mathrm{CO}_{2}$ emissions include emissions and removals of $\mathrm{CO}_{2}$ from the AFOLU sector, including land under forestry and, in some assessments, $\mathrm{CO}_{2}$ sinks in agricultural soils.

22 A majority of the Earth System Models assessed in WGI project a continued land carbon uptake under all RCPs through to 2100, but some models simulate a land carbon loss due to the combined effect of climate change and land-use change. [WGI SPM.E.7, WGI 6.4] 
Direct Sectoral $\mathrm{CO}_{2}$ and Non- $\mathrm{CO}_{2}$ GHG Emissions in Baseline and Mitigation Scenarios with and without CCS
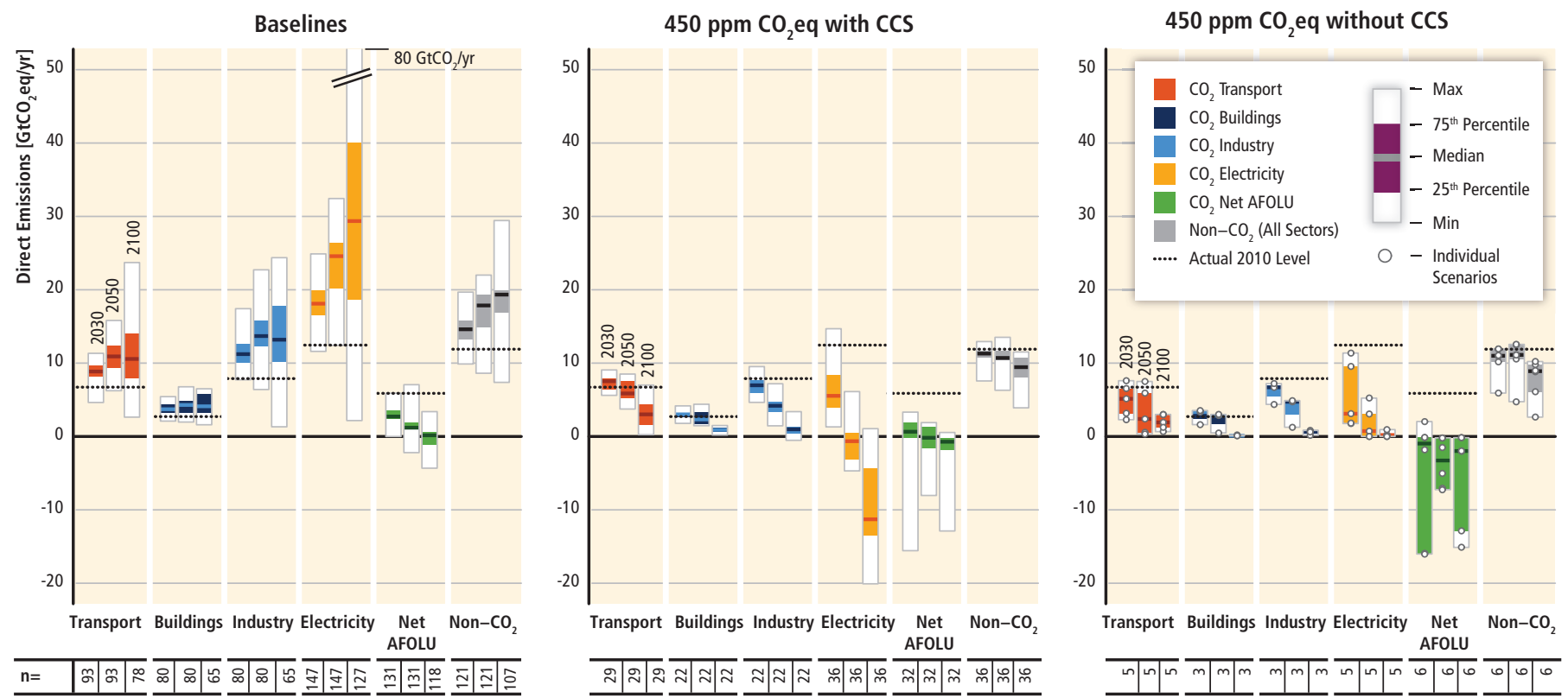

Figure SPM.7| Direct emissions of $\mathrm{CO}_{2}$ by sector and total non- $\mathrm{CO}_{2}$ GHGs (Kyoto gases) across sectors in baseline (left panel) and mitigation scenarios that reach around 450 (430-480) ppm CO eq with CCS (middle panel) and without CCS (right panel). The numbers at the bottom of the graphs refer to the number of scenarios included in the range which differs across sectors and time due to different sectoral resolution and time horizon of models. Note that many models cannot reach about $450 \mathrm{ppm} \mathrm{CO}_{2} \mathrm{eq}$ concentration by 2100 in the absence of CCS, resulting in a low number of scenarios for the right panel. [Figures 6.34 and 6.35 ]

Infrastructure developments and long-lived products that lock societies into GHG-intensive emissions pathways may be difficult or very costly to change, reinforcing the importance of early action for ambitious mitigation (robust evidence, high agreement). This lock-in risk is compounded by the lifetime of the infrastructure, by the difference in emissions associated with alternatives, and the magnitude of the investment cost. As a result, lock-in related to infrastructure and spatial planning is the most difficult to reduce. However, materials, products and infrastructure with long lifetimes and low lifecycle emissions can facilitate a transition to low-emission pathways while also reducing emissions through lower levels of material use. [5.6.3, 6.3.6.4, 9.4, 10.4, 12.3, 12.4]

There are strong interdependencies in mitigation scenarios between the pace of introducing mitigation measures in energy supply and energy end-use and developments in the AFOLU sector (high confidence). The distribution of the mitigation effort across sectors is strongly influenced by the availability and performance of BECCS and large scale afforestation (Figure SPM.7). This is particularly the case in scenarios reaching $\mathrm{CO}_{2}$ eq concentrations of about $450 \mathrm{ppm}$ by 2100 . Well-designed systemic and cross-sectoral mitigation strategies are more cost-effective in cutting emissions than a focus on individual technologies and sectors. At the energy system level these include reductions in the GHG emission intensity of the energy supply sector, a switch to low-carbon energy carriers (including low-carbon electricity) and reductions in energy demand in the end-use sectors without compromising development (Figure SPM.8). [6.3.5, 6.4, 6.8, 7.11, Table TS.2]

Mitigation scenarios reaching around $450 \mathrm{ppm} \mathrm{CO}_{2}$ eq concentrations by 2100 show large-scale global changes in the energy supply sector (robust evidence, high agreement). In these selected scenarios, global $\mathrm{CO}_{2}$ emissions from the energy supply sector are projected to decline over the next decades and are characterized by reductions of $90 \%$ or more below 2010 levels between 2040 and 2070. Emissions in many of these scenarios are projected to decline to below zero thereafter. $[6.3 .4,6.8,7.1,7.11]$ 
Final Energy Demand Reduction and Low-Carbon Energy Carrier Shares in Energy End-Use Sectors
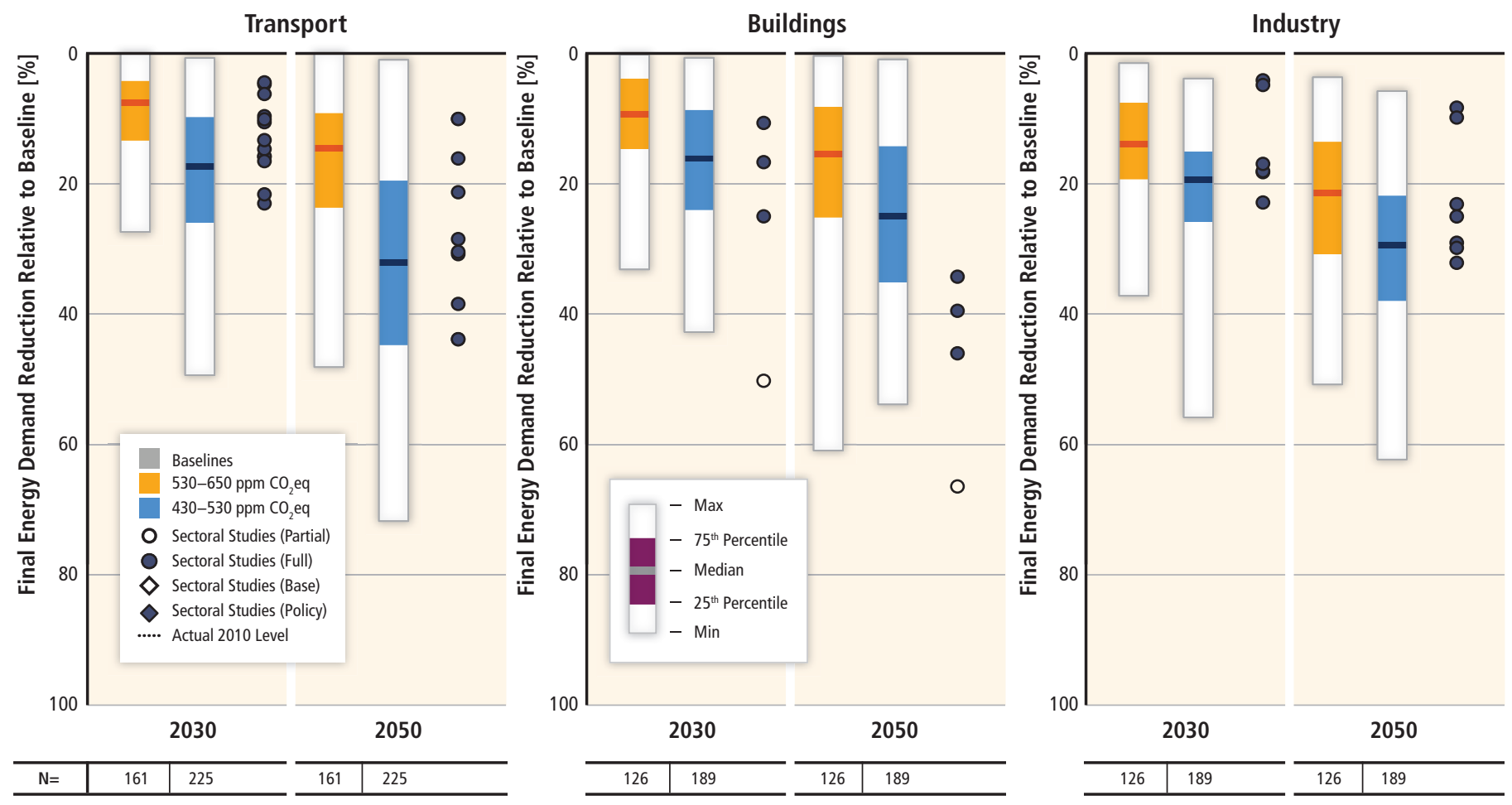

Transport

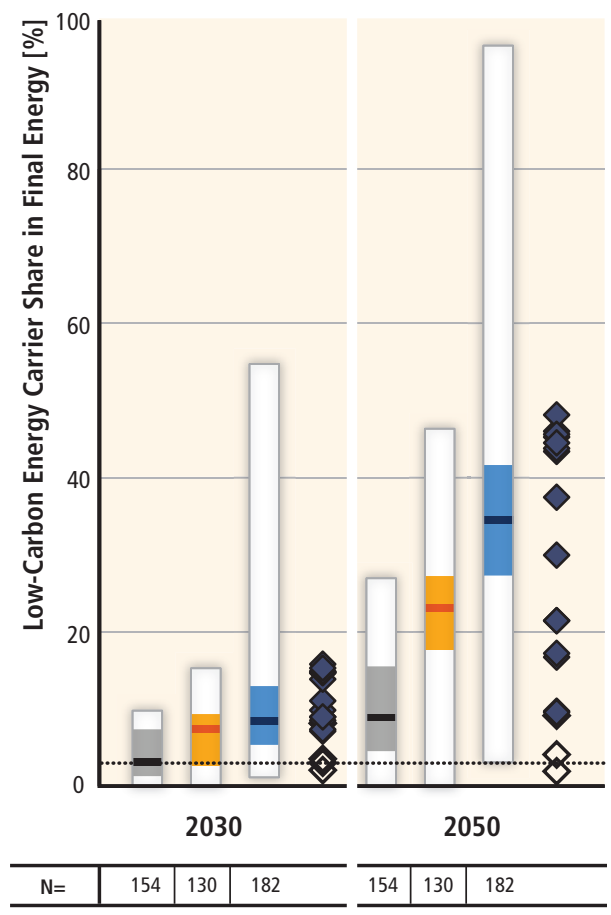

Buildings

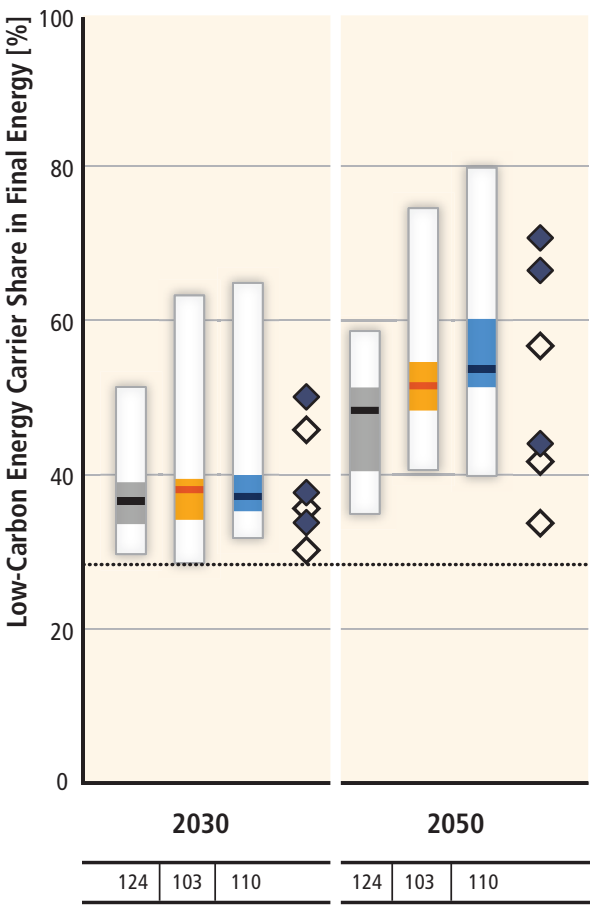

Industry

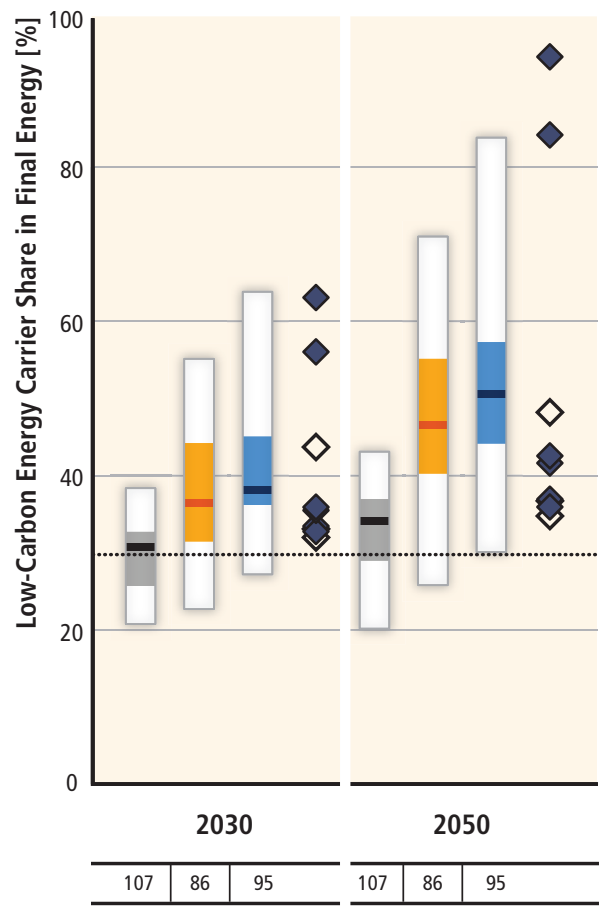

Figure SPM.8| Final energy demand reduction relative to baseline (upper row) and low-carbon energy carrier shares in final energy (lower row) in the transport, buildings, and industry sectors by 2030 and 2050 in scenarios from two different $\mathrm{CO}_{2}$ eq concentration categories compared to sectoral studies assessed in Chapters 8-10. The demand reductions shown by these scenarios do not compromise development. Low-carbon energy carriers include electricity, hydrogen and liquid biofuels in transport, electricity in buildings and electricity, heat, hydrogen and bioenergy in industry. The numbers at the bottom of the graphs refer to the number of scenarios included in the ranges which differ across sectors and time due to different sectoral resolution and time horizon of models. [Figures 6.37 and 6.38] 
Efficiency enhancements and behavioural changes, in order to reduce energy demand compared to baseline scenarios without compromising development, are a key mitigation strategy in scenarios reaching atmospheric $\mathrm{CO}_{2}$ eq concentrations of about 450 to about $500 \mathrm{ppm}$ by 2100 (robust evidence, high agreement). Near-term reductions in energy demand are an important element of cost-effective mitigation strategies, provide more flexibility for reducing carbon intensity in the energy supply sector, hedge against related supply-side risks, avoid lock-in to carbon-intensive infrastructures, and are associated with important co-benefits. Both integrated and sectoral studies provide similar estimates for energy demand reductions in the transport, buildings and industry sectors for 2030 and 2050 (Figure SPM.8). [6.3.4, 6.6, 6.8, 7.11, 8.9, 9.8, 10.10]

Behaviour, lifestyle and culture have a considerable influence on energy use and associated emissions, with high mitigation potential in some sectors, in particular when complementing technological and structural change ${ }^{23}$ (medium evidence, medium agreement). Emissions can be substantially lowered through changes in consumption patterns (e. g., mobility demand and mode, energy use in households, choice of longer-lasting products) and dietary change and reduction in food wastes. A number of options including monetary and non-monetary incentives as well as information measures may facilitate behavioural changes. [6.8, 7.9, 8.3.5, 8.9, 9.2, 9.3, 9.10, Box 10.2, 10.4, 11.4, 12.4, $12.6,12.7,15.3,15.5$, Table TS.2]

In the baseline scenarios assessed in AR5, direct $\mathrm{CO}_{2}$ emissions from the energy supply sector are projected to almost double or even triple by 2050 compared to the level of $14.4 \mathrm{GtCO}_{2} /$ year in 2010 , unless energy intensity improvements can be significantly accelerated beyond the historical development (medium evidence, medium agreement). In the last decade, the main contributors to emission growth were a growing energy demand and an increase of the share of coal in the global fuel mix. The availability of fossil fuels alone will not be sufficient to limit $\mathrm{CO}_{2}$ eq concentration to levels such as $450 \mathrm{ppm}, 550 \mathrm{ppm}$, or $650 \mathrm{ppm}$. (Figure SPM.7) [6.3.4, 7.2, 7.3, Figures 6.15, TS.15]

Decarbonizing (i. e. reducing the carbon intensity of) electricity generation is a key component of costeffective mitigation strategies in achieving low-stabilization levels $\left(430-530 \mathrm{ppm} \mathrm{CO}{ }_{2} \mathrm{eq}\right)$; in most integrated modelling scenarios, decarbonization happens more rapidly in electricity generation than in the industry, buildings, and transport sectors (medium evidence, high agreement) (Figure SPM.7). In the majority of low-stabilization scenarios, the share of low-carbon electricity supply (comprising renewable energy (RE), nuclear and CCS) increases from the current share of approximately $30 \%$ to more than $80 \%$ by 2050 , and fossil fuel power generation without CCS is phased out almost entirely by 2100 (Figure SPM. 7). [6.8, 7.11, Figures 7.14, TS.18]

Since AR4, many RE technologies have demonstrated substantial performance improvements and cost reductions, and a growing number of RE technologies have achieved a level of maturity to enable deployment at significant scale (robust evidence, high agreement). Regarding electricity generation alone, RE accounted for just over half of the new electricity-generating capacity added globally in 2012, led by growth in wind, hydro and solar power. However, many RE technologies still need direct and/or indirect support, if their market shares are to be significantly increased; RE technology policies have been successful in driving recent growth of RE. Challenges for integrating RE into energy systems and the associated costs vary by RE technology, regional circumstances, and the characteristics of the existing background energy system (medium evidence, medium agreement). [7.5.3, 7.6.1, 7.8.2, 7.12, Table 7.1]

Nuclear energy is a mature low-GHG emission source of baseload power, but its share of global electricity generation has been declining (since 1993). Nuclear energy could make an increasing contribution to lowcarbon energy supply, but a variety of barriers and risks exist (robust evidence, high agreement). Those include:

23 Structural changes refer to systems transformations whereby some components are either replaced or potentially substituted by other components (see WGIII AR5 Glossary). 
operational risks, and the associated concerns, uranium mining risks, financial and regulatory risks, unresolved waste management issues, nuclear weapon proliferation concerns, and adverse public opinion (robust evidence, high agreement). New fuel cycles and reactor technologies addressing some of these issues are being investigated and progress in research and development has been made concerning safety and waste disposal. [7.5.4, 7.8, 7.9, 7.12, Figure TS.19]

GHG emissions from energy supply can be reduced significantly by replacing current world average coal-fired power plants with modern, highly efficient natural gas combined-cycle power plants or combined heat and power plants, provided that natural gas is available and the fugitive emissions associated with extraction and supply are low or mitigated (robust evidence, high agreement). In mitigation scenarios reaching about $450 \mathrm{ppm}$ $\mathrm{CO}_{2}$ eq concentrations by 2100 , natural gas power generation without CCS acts as a bridge technology, with deployment increasing before peaking and falling to below current levels by 2050 and declining further in the second half of the century (robust evidence, high agreement). [7.5.1, 7.8, 7.9, 7.11, 7.12]

Carbon dioxide capture and storage (CCS) technologies could reduce the lifecycle GHG emissions of fossil fuel power plants (medium evidence, medium agreement). While all components of integrated CCS systems exist and are in use today by the fossil fuel extraction and refining industry, CCS has not yet been applied at scale to a large, operational commercial fossil fuel power plant. CCS power plants could be seen in the market if this is incentivized by regulation and/or if they become competitive with their unabated counterparts, for instance, if the additional investment and operational costs, caused in part by efficiency reductions, are compensated by sufficiently high carbon prices (or direct financial support). For the large-scale future deployment of CCS, well-defined regulations concerning short- and long-term responsibilities for storage are needed as well as economic incentives. Barriers to large-scale deployment of CCS technologies include concerns about the operational safety and long-term integrity of $\mathrm{CO}_{2}$ storage as well as transport risks. There is, however, a growing body of literature on how to ensure the integrity of $\mathrm{CO}_{2}$ wells, on the potential consequences of a pressure build-up within a geologic formation caused by $\mathrm{CO}_{2}$ storage (such as induced seismicity), and on the potential human health and environmental impacts from $\mathrm{CO}_{2}$ that migrates out of the primary injection zone (limited evidence, medium agreement). [7.5.5., 7.8, 7.9, 7.11, 7.12, 11.13]

Combining bioenergy with CCS (BECCS) offers the prospect of energy supply with large-scale net negative emissions which plays an important role in many low-stabilization scenarios, while it entails challenges and risks (limited evidence, medium agreement). These challenges and risks include those associated with the upstream large-scale provision of the biomass that is used in the CCS facility as well as those associated with the CCS technology itself. [7.5.5, 7.9, 11.13]

SPM.4.2.3

Energy end-use sectors

Transport

The transport sector accounted for $27 \%$ of final energy use and $6.7 \mathrm{GtCO}_{2}$ direct emissions in 2010, with baseline $\mathrm{CO}_{2}$ emissions projected to approximately double by 2050 (medium evidence, medium agreement). This growth in $\mathrm{CO}_{2}$ emissions from increasing global passenger and freight activity could partly offset future mitigation measures that include fuel carbon and energy intensity improvements, infrastructure development, behavioural change and comprehensive policy implementation (high confidence). Overall, reductions in total transport $\mathrm{CO}_{2}$ emissions of $15-40 \%$ compared to baseline growth could be achieved in 2050 (medium evidence, medium agreement). (Figure SPM.7) [6.8, $8.1,8.2,8.9,8.10]$

Technical and behavioural mitigation measures for all transport modes, plus new infrastructure and urban redevelopment investments, could reduce final energy demand in 2050 by around $40 \%$ below the baseline, with the mitigation potential assessed to be higher than reported in the AR4 (robust evidence, medium agreement). Projected energy efficiency and vehicle performance improvements range from 30-50\% in 2030 relative to 2010 depending on transport mode and vehicle type (medium evidence, medium agreement). Integrated urban planning, 
transit-oriented development, more compact urban form that supports cycling and walking, can all lead to modal shifts as can, in the longer term, urban redevelopment and investments in new infrastructure such as high-speed rail systems that reduce short-haul air travel demand (medium evidence, medium agreement). Such mitigation measures are challenging, have uncertain outcomes, and could reduce transport GHG emissions by $20-50 \%$ in 2050 compared to baseline (limited evidence, low agreement). (Figure SPM.8 upper panel) [8.2, 8.3, 8.4, 8.5, 8.6, 8.7, 8.8, 8.9, 12.4, 12.5]

Strategies to reduce the carbon intensities of fuel and the rate of reducing carbon intensity are constrained by challenges associated with energy storage and the relatively low energy density of low-carbon transport fuels (medium confidence). Integrated and sectoral studies broadly agree that opportunities for switching to low-carbon fuels exist in the near term and will grow over time. Methane-based fuels are already increasing their share for road vehicles and waterborne craft. Electricity produced from low-carbon sources has near-term potential for electric rail and short- to medium-term potential as electric buses, light-duty and 2-wheel road vehicles are deployed. Hydrogen fuels from low-carbon sources constitute longer-term options. Commercially available liquid and gaseous biofuels already provide co-benefits together with mitigation options that can be increased by technology advances. Reducing transport emissions of particulate matter (including black carbon), tropospheric ozone and aerosol precursors (including $\mathrm{NO}_{x}$ ) can have human health and mitigation co-benefits in the short term (medium evidence, medium agreement). [8.2, 8.3, 11.13, Figure TS.20, right panel]

The cost-effectiveness of different carbon reduction measures in the transport sector varies significantly with vehicle type and transport mode (high confidence). The levelized costs of conserved carbon can be very low or negative for many short-term behavioural measures and efficiency improvements for light- and heavy-duty road vehicles and waterborne craft. In 2030, for some electric vehicles, aircraft and possibly high-speed rail, levelized costs could be more than USD100/tCO ${ }_{2}$ avoided (limited evidence, medium agreement). [8.6, 8.8, 8.9, Figures TS.21, TS.22]

Regional differences influence the choice of transport mitigation options (high confidence). Institutional, legal, financial and cultural barriers constrain low-carbon technology uptake and behavioural change. Established infrastructure may limit the options for modal shift and lead to a greater reliance on advanced vehicle technologies; a slowing of growth in light-duty vehicle demand is already evident in some OECD countries. For all economies, especially those with high rates of urban growth, investment in public transport systems and low-carbon infrastructure can avoid lock-in to carbon-intensive modes. Prioritizing infrastructure for pedestrians and integrating non-motorized and transit services can create economic and social co-benefits in all regions (medium evidence, medium agreement). [8.4, 8.8, 8.9, 14.3, Table 8.3]

Mitigation strategies, when associated with non-climate policies at all government levels, can help decouple transport GHG emissions from economic growth in all regions (medium confidence). These strategies can help reduce travel demand, incentivise freight businesses to reduce the carbon intensity of their logistical systems and induce modal shifts, as well as provide co-benefits including improved access and mobility, better health and safety, greater energy security, and cost and time savings (medium evidence, high agreement). [8.7, 8.10]

\section{Buildings}

In 2010, the buildings sector ${ }^{24}$ accounted for around $32 \%$ final energy use and $8.8 \mathrm{GtCO}_{2}$ emissions, including direct and indirect emissions, with energy demand projected to approximately double and $\mathrm{CO}_{2}$ emissions to increase by $50-150 \%$ by mid-century in baseline scenarios (medium evidence, medium agreement). This energy demand growth results from improvements in wealth, lifestyle change, access to modern energy services and adequate housing, and urbanisation. There are significant lock-in risks associated with the long lifespans of buildings and related infrastructure, and these are especially important in regions with high construction rates (robust evidence, high agreement). (Figure SPM.7) [9.4]

24 The buildings sector covers the residential, commercial, public and services sectors; emissions from construction are accounted for in the industry sector. 
Recent advances in technologies, know-how and policies provide opportunities to stabilize or reduce global buildings sector energy use by mid-century (robust evidence, high agreement). For new buildings, the adoption of very low energy building codes is important and has progressed substantially since AR4. Retrofits form a key part of the mitigation strategy in countries with established building stocks, and reductions of heating/cooling energy use by $50-90 \%$ in individual buildings have been achieved. Recent large improvements in performance and costs make very low energy construction and retrofits economically attractive, sometimes even at net negative costs. [9.3]

Lifestyle, culture and behaviour significantly influence energy consumption in buildings (limited evidence, high agreement). A three- to five-fold difference in energy use has been shown for provision of similar building-related energy service levels in buildings. For developed countries, scenarios indicate that lifestyle and behavioural changes could reduce energy demand by up to $20 \%$ in the short term and by up to $50 \%$ of present levels by mid-century. In developing countries, integrating elements of traditional lifestyles into building practices and architecture could facilitate the provision of high levels of energy services with much lower energy inputs than baseline. [9.3]

Most mitigation options for buildings have considerable and diverse co-benefits in addition to energy cost savings (robust evidence, high agreement). These include improvements in energy security, health (such as from cleaner wood-burning cookstoves), environmental outcomes, workplace productivity, fuel poverty reductions and net employment gains. Studies which have monetized co-benefits often find that these exceed energy cost savings and possibly climate benefits (medium evidence, medium agreement). [9.6, 9.7, 3.6.3]

Strong barriers, such as split incentives (e. g., tenants and builders), fragmented markets and inadequate access to information and financing, hinder the market-based uptake of cost-effective opportunities. Barriers can be overcome by policy interventions addressing all stages of the building and appliance lifecycles (robust evidence, high agreement). [9.8, 9.10, 16, Box 3.10]

The development of portfolios of energy efficiency policies and their implementation has advanced considerably since AR4. Building codes and appliance standards, if well designed and implemented, have been among the most environmentally and cost-effective instruments for emission reductions (robust evidence, high agreement). In some developed countries they have contributed to a stabilization of, or reduction in, total energy demand for buildings. Substantially strengthening these codes, adopting them in further jurisdictions, and extending them to more building and appliance types, will be a key factor in reaching ambitious climate goals. [9.10, 2.6.5.3]

Industry

In 2010, the industry sector accounted for around $28 \%$ of final energy use, and $13 \mathrm{GtCO}_{2}$ emissions, including direct and indirect emissions as well as process emissions, with emissions projected to increase by $50-150 \%$ by 2050 in the baseline scenarios assessed in AR5, unless energy efficiency improvements are accelerated significantly (medium evidence, medium agreement). Emissions from industry accounted for just over $30 \%$ of global GHG emissions in 2010 and are currently greater than emissions from either the buildings or transport end-use sectors. (Figures SPM.2, SPM.7) [10.3]

The energy intensity of the industry sector could be directly reduced by about $25 \%$ compared to the current level through the wide-scale upgrading, replacement and deployment of best available technologies, particularly in countries where these are not in use and in non-energy intensive industries (high agreement, robust evidence). Additional energy intensity reductions of about $20 \%$ may potentially be realized through innovation (limited evidence, medium agreement). Barriers to implementing energy efficiency relate largely to initial investment costs and lack of information. Information programmes are a prevalent approach for promoting energy efficiency, followed by economic instruments, regulatory approaches and voluntary actions. [10.7, 10.9, 10.11] 
Improvements in GHG emission efficiency and in the efficiency of material use, recycling and re-use of materials and products, and overall reductions in product demand (e. g., through a more intensive use of products) and service demand could, in addition to energy efficiency, help reduce GHG emissions below the baseline level in the industry sector (medium evidence, high agreement). Many emission-reducing options are cost-effective, profitable and associated with multiple co-benefits (better environmental compliance, health benefits etc.). In the long term, a shift to low-carbon electricity, new industrial processes, radical product innovations (e. g., alternatives to cement), or CCS (e.g., to mitigate process emissions) could contribute to significant GHG emission reductions. Lack of policy and experiences in material and product service efficiency are major barriers. [10.4, 10.7, 10.8, 10.11]

$\mathrm{CO}_{2}$ emissions dominate GHG emissions from industry, but there are also substantial mitigation opportunities for non- $\mathrm{CO}_{2}$ gases (robust evidence, high agreement). $\mathrm{CH}_{4}, \mathrm{~N}_{2} \mathrm{O}$ and fluorinated gases from industry accounted for emissions of $0.9 \mathrm{GtCO}_{2}$ eq in 2010. Key mitigation opportunities include, e. g., the reduction of hydrofluorocarbon emissions by process optimization and refrigerant recovery, recycling and substitution, although there are barriers. [Tables 10.2, 10.7]

Systemic approaches and collaborative activities across companies and sectors can reduce energy and material consumption and thus GHG emissions (robust evidence, high agreement). The application of cross-cutting technologies (e. g., efficient motors) and measures (e. g., reducing air or steam leaks) in both large energy intensive industries and small and medium enterprises can improve process performance and plant efficiency cost-effectively. Cooperation across companies (e. g., in industrial parks) and sectors could include the sharing of infrastructure, information, and waste heat utilization. [10.4, 10.5]

Important options for mitigation in waste management are waste reduction, followed by re-use, recycling and energy recovery (robust evidence, high agreement). Waste and wastewater accounted for $1.5 \mathrm{GtCO}_{2} \mathrm{eq}$ in 2010. As the share of recycled or reused material is still low (e. g., globally, around $20 \%$ of municipal solid waste is recycled), waste treatment technologies and recovering energy to reduce demand for fossil fuels can result in significant direct emission reductions from waste disposal. [10.4, 10.14]

The AFOLU sector accounts for about a quarter ( 10-12 $\left.\mathrm{GtCO}_{2} \mathrm{eq} / \mathrm{yr}\right)$ of net anthropogenic GHG emissions mainly from deforestation, agricultural emissions from soil and nutrient management and livestock (medium evidence, high agreement). Most recent estimates indicate a decline in $\mathrm{AFOLU} \mathrm{CO}_{2}$ fluxes, largely due to decreasing deforestation rates and increased afforestation. However, the uncertainty in historical net AFOLU emissions is larger than for other sectors, and additional uncertainties in projected baseline net AFOLU emissions exist. Nonetheless, in the future, net annual baseline $\mathrm{CO}_{2}$ emissions from AFOLU are projected to decline, with net emissions potentially less than half the 2010 level by 2050 and the possibility of the AFOLU sectors becoming a net $\mathrm{CO}_{2}$ sink before the end of century (medium evidence, high agreement). (Figure SPM. 7) [6.3.1.4, 11.2, Figure 6.5]

AFOLU plays a central role for food security and sustainable development. The most cost-effective mitigation options in forestry are afforestation, sustainable forest management and reducing deforestation, with large differences in their relative importance across regions. In agriculture, the most cost-effective mitigation options are cropland management, grazing land management, and restoration of organic soils (medium evidence, high agreement). The economic mitigation potential of supply-side measures is estimated to be 7.2 to 11 $\mathrm{GtCO}_{2}$ eq/year ${ }^{25}$ in 2030 for mitigation efforts consistent with carbon prices ${ }^{26}$ up to $100 \mathrm{USD} / \mathrm{tCO}_{2}$ eq, about a third of which can be achieved at a $<20 \mathrm{USD} / \mathrm{tCO}_{2} \mathrm{eq}$ (medium evidence, medium agreement). There are potential barriers to

\footnotetext{
Full range of all studies: $0.49-11 \mathrm{GtCO}_{2}$ eq/year

In many models that are used to assess the economic costs of mitigation, carbon price is used as a proxy to represent the level of effort in mitigation policies (see WGIII AR5 Glossary).
} 
implementation of available mitigation options $[11.7,11.8]$. Demand-side measures, such as changes in diet and reductions of losses in the food supply chain, have a significant, but uncertain, potential to reduce GHG emissions from food production (medium evidence, medium agreement). Estimates vary from roughly $0.76-8.6 \mathrm{GtCO}_{2} \mathrm{eq} / \mathrm{yr}$ by 2050 (limited evidence, medium agreement). [11.4, 11.6, Figure 11.14]

Policies governing agricultural practices and forest conservation and management are more effective when involving both mitigation and adaptation. Some mitigation options in the AFOLU sector (such as soil and forest carbon stocks) may be vulnerable to climate change (medium evidence, high agreement). When implemented sustainably, activities to reduce emissions from deforestation and forest degradation (REDD $+{ }^{27}$ is an example designed to be sustainable) are cost-effective policy options for mitigating climate change, with potential economic, social and other environmental and adaptation co-benefits (e. g., conservation of biodiversity and water resources, and reducing soil erosion) (limited evidence, medium agreement). [11.3.2, 11.10]

Bioenergy can play a critical role for mitigation, but there are issues to consider, such as the sustainability of practices and the efficiency of bioenergy systems (robust evidence, medium agreement) [11.4.4, Box 11.5, 11.13.6, 11.13.7]. Barriers to large-scale deployment of bioenergy include concerns about GHG emissions from land, food security, water resources, biodiversity conservation and livelihoods. The scientific debate about the overall climate impact related to land-use competition effects of specific bioenergy pathways remains unresolved (robust evidence, high agreement). $[11.4 .4,11.13]$ Bioenergy technologies are diverse and span a wide range of options and technology pathways. Evidence suggests that options with low lifecycle emissions (e. g., sugar cane, Miscanthus, fast growing tree species, and sustainable use of biomass residues), some already available, can reduce GHG emissions; outcomes are site-specific and rely on efficient integrated 'biomass-to-bioenergy systems', and sustainable land-use management and governance. In some regions, specific bioenergy options, such as improved cookstoves, and small-scale biogas and biopower production, could reduce GHG emissions and improve livelihoods and health in the context of sustainable development (medium evidence, medium agreement). [11.13]

Urbanization is a global trend and is associated with increases in income, and higher urban incomes are correlated with higher consumption of energy and GHG emissions (medium evidence, high agreement). As of 2011, more than $52 \%$ of the global population lives in urban areas. In 2006 , urban areas accounted for $67-76 \%$ of energy use and $71-76 \%$ of energy-related $\mathrm{CO}_{2}$ emissions. By 2050 , the urban population is expected to increase to 5.6-7.1 billion, or $64-69 \%$ of world population. Cities in non-Annex I countries generally have higher levels of energy use compared to the national average, whereas cities in Annex I countries generally have lower energy use per capita than national averages (medium evidence, medium agreement). [12.2, 12.3]

The next two decades present a window of opportunity for mitigation in urban areas, as a large portion of the world's urban areas will be developed during this period (limited evidence, high agreement). Accounting for trends in declining population densities, and continued economic and population growth, urban land cover is projected to expand by $56-310 \%$ between 2000 and 2030. [12.2, 12.3, 12.4, 12.8]

Mitigation options in urban areas vary by urbanization trajectories and are expected to be most effective when policy instruments are bundled (robust evidence, high agreement). Infrastructure and urban form are strongly interlinked, and lock-in patterns of land use, transport choice, housing, and behaviour. Effective mitigation strategies involve packages of mutually reinforcing policies, including co-locating high residential with high employment densities,

27 See WGIII AR5 Glossary. 
achieving high diversity and integration of land uses, increasing accessibility and investing in public transport and other demand management measures. $[8.4,12.3,12.4,12.5,12.6]$

The largest mitigation opportunities with respect to human settlements are in rapidly urbanizing areas where urban form and infrastructure are not locked in, but where there are often limited governance, technical, financial, and institutional capacities (robust evidence, high agreement). The bulk of urban growth is expected in small- to medium-size cities in developing countries. The feasibility of spatial planning instruments for climate change mitigation is highly dependent on a city's financial and governance capability. $[12.6,12.7]$

Thousands of cities are undertaking climate action plans, but their aggregate impact on urban emissions is uncertain (robust evidence, high agreement). There has been little systematic assessment on their implementation, the extent to which emission reduction targets are being achieved, or emissions reduced. Current climate action plans focus largely on energy efficiency. Fewer climate action plans consider land-use planning strategies and cross-sectoral measures to reduce sprawl and promote transit-oriented development ${ }^{28}$. $[12.6,12.7,12.9]$

Successful implementation of urban-scale climate change mitigation strategies can provide co-benefits (robust evidence, high agreement). Urban areas throughout the world continue to struggle with challenges, including ensuring access to energy, limiting air and water pollution, and maintaining employment opportunities and competitiveness. Action on urban-scale mitigation often depends on the ability to relate climate change mitigation efforts to local co-benefits (robust evidence, high agreement). [12.5, 12.6, 12.7, 12.8]

\section{SPM.5 Mitigation policies and institutions}

SPM.5.1

\section{Sectoral and national policies}

Substantial reductions in emissions would require large changes in investment patterns. Mitigation scenarios in which policies stabilize atmospheric concentrations (without overshoot) in the range from 430 to 530 ppm $\mathrm{CO}_{2}$ eq by 2100 lead to substantial shifts in annual investment flows during the period 2010-2029 compared to baseline scenarios (Figure SPM.9). Over the next two decades (2010 to 2029), annual investment in conventional fossil fuel technologies associated with the electricity supply sector is projected to decline by about 30 (2-166) billion USD (median: $-20 \%$ compared to 2010) while annual investment in low-carbon electricity supply (i.e., renewables, nuclear and electricity generation with CCS) is projected to rise by about 147 (31-360) billion USD (median: $+100 \%$ compared to 2010) (limited evidence, medium agreement). For comparison, global total annual investment in the energy system is presently about 1200 billion USD. In addition, annual incremental energy efficiency investments in transport, buildings and industry is projected to increase by about 336 (1-641) billion USD (limited evidence, medium agreement), frequently involving modernization of existing equipment. [13.11, 16.2.2]

There is no widely agreed definition of what constitutes climate finance, but estimates of the financial flows associated with climate change mitigation and adaptation are available. Published assessments of all current annual financial flows whose expected effect is to reduce net GHG emissions and/or to enhance resilience to climate change and climate variability show 343 to 385 billion USD per year globally (medium confidence) [Box TS.14]. Most of this goes to mitigation. Out of this, total public climate finance that flowed to developing countries is estimated to be between 35 and 49 billion USD/yr in 2011 and 2012 (medium confidence). Estimates of international private climate

28 See WGIII AR5 Glossary. 
Change in Annual Investment Flows from Baseline Levels

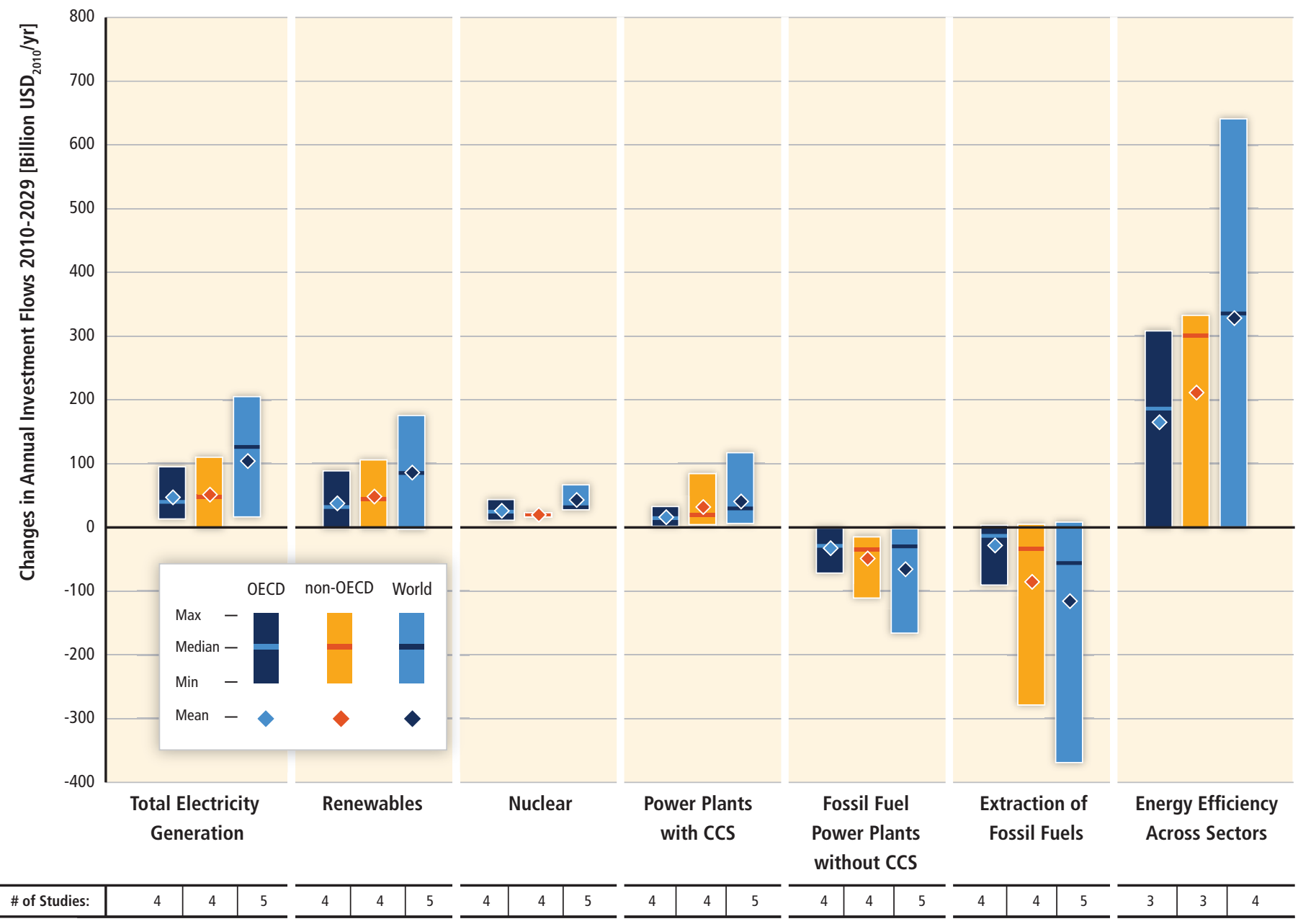

Figure SPM.9| Change in annual investment flows from the average baseline level over the next two decades (2010-2029) for mitigation scenarios that stabilize concentrations within the range of approximately $430-530 \mathrm{ppm} \mathrm{CO}$ eq by 2100 . Investment changes are based on a limited number of model studies and model comparisons. Total electricity generation (leftmost column) is the sum of renewables, nuclear, power plants with CCS and fossil fuel power plants without CCS. The vertical bars indicate the range between minimum and maximum estimate; the horizontal bar indicates the median. Proximity to this median value does not imply higher likelihood because of the different degree of aggregation of model results, the low number of studies available and different assumptions in the different studies considered. The numbers in the bottom row show the total number of studies in the literature used for the assessment. This underscores that investment needs are still an evolving area of research that relatively few studies have examined. [Figure 16.3]

finance flowing to developing countries range from 10 to 72 billion USD/yr including foreign direct investment as equity and loans in the range of 10 to 37 billion USD/yr over the period of 2008-2011 (medium confidence). [16.2.2]

There has been a considerable increase in national and sub-national mitigation plans and strategies since AR4. In $2012,67 \%$ of global GHG emissions were subject to national legislation or strategies versus $45 \%$ in 2007 . However, there has not yet been a substantial deviation in global emissions from the past trend [Figure 1.3c]. These plans and strategies are in their early stages of development and implementation in many countries, making it difficult to assess their aggregate impact on future global emissions (medium evidence, high agreement). [14.3.4, 14.3.5, 15.1, 15.2]

Since AR4, there has been an increased focus on policies designed to integrate multiple objectives, increase co-benefits and reduce adverse side-effects (high confidence). Governments often explicitly reference co-benefits in climate and sectoral plans and strategies. The scientific literature has sought to assess the size of co-benefits (see Section SPM.4.1) and the greater political feasibility and durability of policies that have large co-benefits and small adverse 
side-effects. $[4.8,5.7,6.6,13.2,15.2]$ Despite the growing attention in policymaking and the scientific literature since AR4, the analytical and empirical underpinnings for understanding many of the interactive effects are under-developed [1.2, $3.6 .3,4.2,4.8,5.7,6.6]$.

Sector-specific policies have been more widely used than economy-wide policies (medium evidence, high agreement). Although most economic theory suggests that economy-wide policies for the singular objective of mitigation would be more cost-effective than sector-specific policies, since AR4 a growing number of studies has demonstrated that administrative and political barriers may make economy-wide policies harder to design and implement than sector-specific policies. The latter may be better suited to address barriers or market failures specific to certain sectors, and may be bundled in packages of complementary policies. [6.3.6.5, 8.10, 9.10, 10.10, 15.2, 15.5, 15.8, 15.9]

Regulatory approaches and information measures are widely used, and are often environmentally effective (medium evidence, medium agreement). Examples of regulatory approaches include energy efficiency standards; examples of information programmes include labelling programmes that can help consumers make better-informed decisions. While such approaches have often been found to have a net social benefit, the scientific literature is divided on the extent to which such policies can be implemented with negative private costs to firms and individuals. [Box 3.10, 15.5.5, 15.5.6] There is general agreement that rebound effects exist, whereby higher efficiency can lead to lower energy prices and greater consumption, but there is low agreement in the literature on the magnitude $[3.9 .5,5.7 .2,14.4 .2,15.5 .4]$.

Since AR4, cap and trade systems for GHGs have been established in a number of countries and regions. Their short-run environmental effect has been limited as a result of loose caps or caps that have not proved to be constraining (limited evidence, medium agreement). This was related to factors such as the financial and economic crisis that reduced energy demand, new energy sources, interactions with other policies, and regulatory uncertainty. In principle, a cap and trade system can achieve mitigation in a cost-effective way; its implementation depends on national circumstances. Though earlier programmes relied almost exclusively on grandfathering (free allocation of permits), auctioning permits is increasingly applied. If allowances are auctioned, revenues can be used to address other investments with a high social return, and/or reduce the tax and debt burden. [14.4.2, 15.5.3]

In some countries, tax-based policies specifically aimed at reducing GHG emissions-alongside technology and other policies - have helped to weaken the link between GHG emissions and GDP (high confidence). In a large group of countries, fuel taxes (although not necessarily designed for the purpose of mitigation) have effects that are akin to sectoral carbon taxes [Table 15.2]. The demand reduction in transport fuel associated with a $1 \%$ price increase is $0.6 \%$ to $0.8 \%$ in the long run, although the short-run response is much smaller [15.5.2]. In some countries revenues are used to reduce other taxes and/or to provide transfers to low-income groups. This illustrates the general principle that mitigation policies that raise government revenue generally have lower social costs than approaches which do not. While it has previously been assumed that fuel taxes in the transport sector are regressive, there have been a number of other studies since AR4 that have shown them to be progressive, particularly in developing countries (medium evidence, medium agreement). [3.6.3, 14.4.2, 15.5.2]

The reduction of subsidies for GHG-related activities in various sectors can achieve emission reductions, depending on the social and economic context (high confidence). While subsidies can affect emissions in many sectors, most of the recent literature has focused on subsidies for fossil fuels. Since AR4 a small but growing literature based on economy-wide models has projected that complete removal of subsidies for fossil fuels in all countries could result in reductions in global aggregate emissions by mid-century (medium evidence, medium agreement) $[7.12,13.13,14.3 .2$, 15.5.2]. Studies vary in methodology, the type and definition of subsidies and the time frame for phase out considered. In particular, the studies assess the impacts of complete removal of all fossil fuel subsidies without seeking to assess which subsidies are wasteful and inefficient, keeping in mind national circumstances. Although political economy barriers are substantial, some countries have reformed their tax and budget systems to reduce fuel subsidies. To help reduce possible adverse effects on lower-income groups who often spend a large fraction of their income on energy services, many governments have utilized lump-sum cash transfers or other mechanisms targeted on the poor. [15.5.2] 
Interactions between or among mitigation policies may be synergistic or may have no additive effect on reducing emissions (medium evidence, high agreement). For instance, a carbon tax can have an additive environmental effect to policies such as subsidies for the supply of RE. By contrast, if a cap and trade system has a binding cap (sufficiently stringent to affect emission-related decisions), then other policies such as RE subsidies have no further impact on reducing emissions within the time period that the cap applies (although they may affect costs and possibly the viability of more stringent future targets) (medium evidence, high agreement). In either case, additional policies may be needed to address market failures relating to innovation and technology diffusion. [15.7]

Some mitigation policies raise the prices for some energy services and could hamper the ability of societies to expand access to modern energy services to underserved populations (low confidence). These potential adverse side-effects can be avoided with the adoption of complementary policies (medium confidence). Most notably, about 1.3 billion people worldwide do not have access to electricity and about 3 billion are dependent on traditional solid fuels for cooking and heating with severe adverse effects on health, ecosystems and development. Providing access to modern energy services is an important sustainable development objective. The costs of achieving nearly universal access to electricity and clean fuels for cooking and heating are projected to be between 72 and 95 billion USD per year until 2030 with minimal effects on GHG emissions (limited evidence, medium agreement). A transition away from the use of traditional biomass $\mathrm{s}^{29}$ and the more efficient combustion of solid fuels reduce air pollutant emissions, such as sulfur dioxide $\left(\mathrm{SO}_{2}\right)$, nitrogen oxides $\left(\mathrm{NO}_{x}\right)$, carbon monoxide $(\mathrm{CO})$, and black carbon $(\mathrm{BC})$, and thus yield large health benefits (high confidence). [4.3, 6.6, 7.9, 9.3, 9.7, 11.13.6, 16.8]

Technology policy complements other mitigation policies (high confidence). Technology policy includes technologypush (e.g., publicly funded R\&D) and demand-pull (e.g., governmental procurement programmes). Such policies address market failures related to innovation and technology diffusion. [3.11, 15.6] Technology support policies have promoted substantial innovation and diffusion of new technologies, but the cost-effectiveness of such policies is often difficult to assess $[2.6 .5,7.12,9.10]$. Nevertheless, program evaluation data can provide empirical evidence on the relative effectiveness of different policies and can assist with policy design [15.6.5].

In many countries, the private sector plays central roles in the processes that lead to emissions as well as to mitigation. Within appropriate enabling environments, the private sector, along with the public sector, can play an important role in financing mitigation (medium evidence, high agreement). The share of total mitigation finance from the private sector, acknowledging data limitations, is estimated to be on average between two-thirds and three-fourths on the global level (2010-2012) (limited evidence, medium agreement). In many countries, public finance interventions by governments and national and international development banks encourage climate investments by the private sector [16.2.1] and provide finance where private sector investment is limited. The quality of a country's enabling environment includes the effectiveness of its institutions, regulations and guidelines regarding the private sector, security of property rights, credibility of policies and other factors that have a substantial impact on whether private firms invest in new technologies and infrastructures [16.3]. Dedicated policy instruments, for example, credit insurance, power purchase agreements and feed-in tariffs, concessional finance or rebates, provide an incentive for investment by lowering risks for private actors [16.4].

29 See WGIII AR5 Glossary. 
SPM.5.2

\section{International cooperation}

The United Nations Framework Convention on Climate Change (UNFCCC) is the main multilateral forum focused on addressing climate change, with nearly universal participation. Other institutions organized at different levels of governance have resulted in diversifying international climate change cooperation. [13.3.1, 13.4.1.4, 13.5]

Existing and proposed international climate change cooperation arrangements vary in their focus and degree of centralization and coordination. They span: multilateral agreements, harmonized national policies and decentralized but coordinated national policies, as well as regional and regionally-coordinated policies. [Figure TS.38, 13.4.1, $13.13 .2,14.4]$

The Kyoto Protocol offers lessons towards achieving the ultimate objective of the UNFCCC, particularly with respect to participation, implementation, flexibility mechanisms, and environmental effectiveness (medium evidence, low agreement). [5.3.3, 13.3.4, 13.7.2, 13.13.1.1, 13.13.1.2, 14.3.7.1, Table TS.9]

UNFCCC activities since 2007 have led to an increasing number of institutions and other arrangements for international climate change cooperation. [13.5.1.1, 13.13.1.3, 16.2.1]

Policy linkages among regional, national, and sub-national climate policies offer potential climate change mitigation and adaptation benefits (medium evidence, medium agreement). Linkages can be established between national policies, various instruments, and through regional cooperation. [13.3.1, 13.5.3, 13.6, 13.7, 13.13.2.3, 14.4, Figure 13.4]

Various regional initiatives between the national and global scales are either being developed or implemented, but their impact on global mitigation has been limited to date (medium confidence). Many climate policies can be more effective if implemented across geographical regions. [13.13, 13.6, 14.4, 14.5] 


\section{Technical Summary}

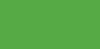





\section{Technical Summary}

\section{Coordinating Lead Authors:}

Ottmar Edenhofer (Germany), Ramón Pichs-Madruga (Cuba), Youba Sokona (Mali/Switzerland), Susanne Kadner (Germany), Jan C. Minx (Germany), Steffen Brunner (Germany)

\section{Lead Authors:}

Shardul Agrawala (France), Giovanni Baiocchi (UK/Italy), Igor Alexeyevich Bashmakov (Russian Federation), Gabriel Blanco (Argentina), John Broome (UK), Thomas Bruckner (Germany), Mercedes Bustamante (Brazil), Leon Clarke (USA), Mariana Conte Grand (Argentina), Felix Creutzig (Germany), Xochitl Cruz-Núñez (Mexico), Shobhakar Dhakal (Nepal/Thailand), Navroz K. Dubash (India), Patrick Eickemeier (Germany), Ellie Farahani (Canada/Switzerland/Germany), Manfred Fischedick (Germany), Marc Fleurbaey (France/USA), Reyer Gerlagh (Netherlands), Luis GómezEcheverri (Austria/Colombia), Sujata Gupta (India/Philippines), Jochen Harnisch (Germany), Kejun Jiang (China), Frank Jotzo (Germany/Australia), Sivan Kartha (USA), Stephan Klasen (Germany), Charles Kolstad (USA), Volker Krey (Austria/Germany), Howard Kunreuther (USA), Oswaldo Lucon (Brazil), Omar Masera (Mexico), Yacob Mulugetta (Ethiopia/UK), Richard Norgaard (USA), Anthony Patt (Austria/Switzerland), Nijavalli H. Ravindranath (India), Keywan Riahi (IIASA/Austria), Joyashree Roy (India), Ambuj Sagar (USA/India), Roberto Schaeffer (Brazil), Steffen Schlömer (Germany), Karen Seto (USA), Kristin Seyboth (USA), Ralph Sims (New Zealand), Pete Smith (UK), Eswaran Somanathan (India), Robert Stavins (USA), Christoph von Stechow (Germany), Thomas Sterner (Sweden), Taishi Sugiyama (Japan), Sangwon Suh (Republic of Korea/USA), Kevin Urama (Nigeria/UK/Kenya), Diana Ürge-Vorsatz (Hungary), Anthony Venables (UK), David G. Victor (USA), Elke Weber (USA), Dadi Zhou (China), Ji Zou (China), Timm Zwickel (Germany)

\section{Contributing Authors:}

Adolf Acquaye (Ghana/UK), Kornelis Blok (Netherlands), Gabriel Chan (USA), Jan Fuglestvedt (Norway), Edgar Hertwich (Austria/Norway), Elmar Kriegler (Germany), Oliver Lah (Germany), Sevastianos Mirasgedis (Greece), Carmenza Robledo Abad (Switzerland/Colombia), Claudia Sheinbaum (Mexico), Steven J. Smith (USA), Detlef van Vuuren (Netherlands)

\section{Review Editors:}

Tomás Hernández-Tejeda (Mexico), Roberta Quadrelli (IEA/Italy) 


\section{This summary should be cited as:}

Edenhofer O., R. Pichs-Madruga, Y. Sokona, S. Kadner, J.C. Minx, S. Brunner, S. Agrawala, G. Baiocchi, I.A. Bashmakov, G. Blanco, J. Broome, T. Bruckner, M. Bustamante, L. Clarke, M. Conte Grand, F. Creutzig, X. Cruz-Núñez, S. Dhakal, N. K. Dubash, P. Eickemeier, E. Farahani, M. Fischedick, M. Fleurbaey, R. Gerlagh, L. Gómez-Echeverri, S. Gupta, J. Harnisch, K. Jiang, F. Jotzo, S. Kartha, S. Klasen, C. Kolstad, V. Krey, H. Kunreuther, O. Lucon, O. Masera, Y. Mulugetta, R. B. Norgaard, A. Patt, N. H. Ravindranath, K. Riahi, J. Roy, A. Sagar, R. Schaeffer, S. Schlömer, K. C. Seto, K. Seyboth, R. Sims, P. Smith, E. Somanathan, R. Stavins, C. von Stechow, T. Sterner, T. Sugiyama, S. Suh, D. Ürge-Vorsatz, K. Urama, A. Venables, D. G. Victor, E. Weber, D. Zhou, J. Zou, and T. Zwickel, 2014: Technical Summary. In: Climate Change 2014: Mitigation of Climate Change. Contribution of Working Group III to the Fifth Assessment Report of the Intergovernmental Panel on Climate Change [Edenhofer, O., R. Pichs-Madruga, Y. Sokona, E. Farahani, S. Kadner, K. Seyboth, A. Adler, I. Baum, S. Brunner, P. Eickemeier, B. Kriemann, J. Savolainen, S. Schlömer, C. von Stechow, T. Zwickel and J. C. Minx (eds.)]. Cambridge University Press, Cambridge, United Kingdom and New York, NY, USA. 


\section{Contents}

TS.1 Introduction and framing

TS.2 Trends in stocks and flows of greenhouse gases and their drivers 41

TS.2.1 Greenhouse gas emission trends 42

TS.2.2 Greenhouse gas emission drivers 47

TS.3 Mitigation pathways and measures in the context of sustainable development 50

TS.3.1 Mitigation pathways .50

TS.3.1.1 Understanding mitigation pathways in the context of multiple objectives .50

TS.3.1.2 Short- and long-term requirements of mitigation pathways 51

TS.3.1.3 Costs, investments and burden sharing .56

TS.3.1.4 Implications of mitigation pathways for other objectives. 61

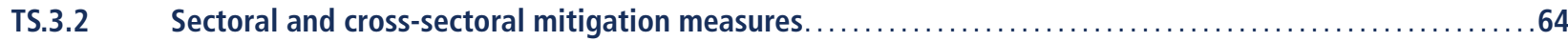

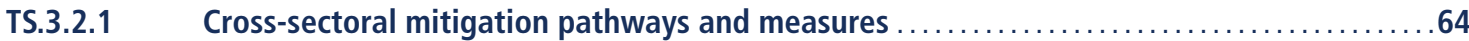

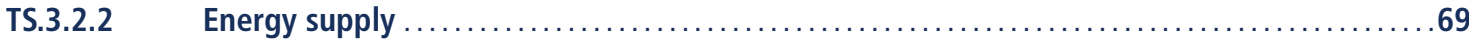

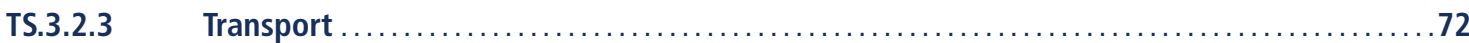

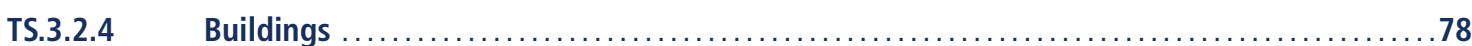

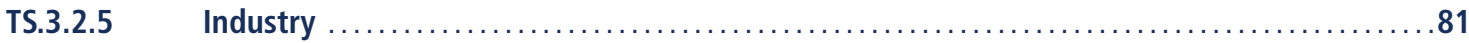

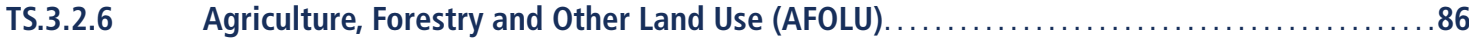

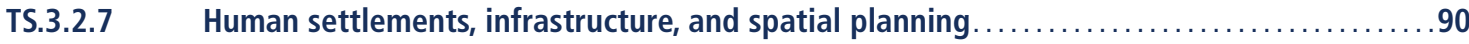




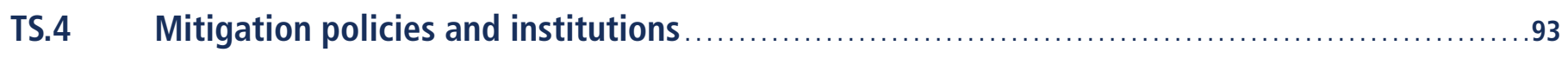

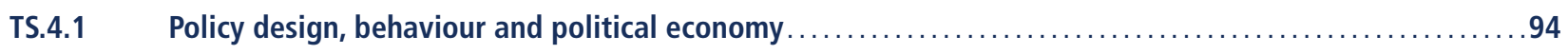

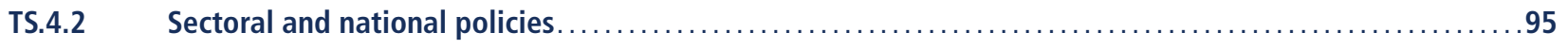

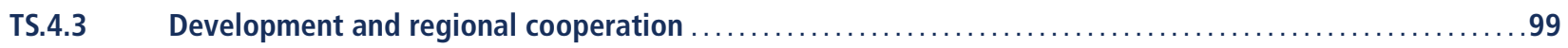

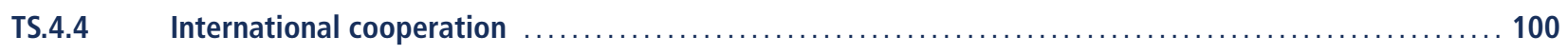

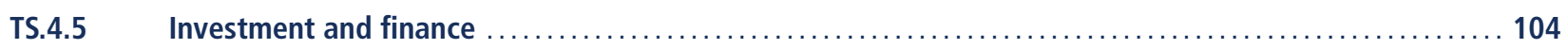




\section{TS.1 Introduction and framing}

'Mitigation', in the context of climate change, is a human intervention to reduce the sources or enhance the sinks of greenhouse gases (GHGs). One of the central messages from Working Groups I and II of the Intergovernmental Panel on Climate Change (IPCC) is that the consequences of unchecked climate change for humans and natural ecosystems are already apparent and increasing. The most vulnerable systems are already experiencing adverse effects. Past GHG emissions have already put the planet on a track for substantial further changes in climate, and while there are many uncertainties in factors such as the sensitivity of the climate system many scenarios lead to substantial climate impacts, including direct harms to human and ecological wellbeing that exceed the ability of those systems to adapt fully.
Because mitigation is intended to reduce the harmful effects of climate change, it is part of a broader policy framework that also includes adaptation to climate impacts. Mitigation, together with adaptation to climate change, contributes to the objective expressed in Article 2 of the United Nations Framework Convention on Climate Change (UNFCCC) to stabilize "greenhouse gas concentrations in the atmosphere at a level to prevent dangerous anthropogenic interference with the climate system $[\ldots]$ within a time frame sufficient to allow ecosystems to adapt $[\ldots]$ to ensure that food production is not threatened and to enable economic development to proceed in a sustainable manner". However, Article 2 is hard to interpret, as concepts such as 'dangerous' and 'sustainable' have different meanings in different decision contexts (see Box TS.1).' Moreover, natural science is unable to predict precisely the response of the climate system to rising GHG

Boxes throughout this summary provide background information on main research concepts and methods that were used to generate insight.

\section{Box TS.1 | Many disciplines aid decision making on climate change}

Something is dangerous if it leads to a significant risk of considerable harm. Judging whether human interference in the climate system is dangerous therefore divides into two tasks. One is to estimate the risk in material terms: what the material consequences of human interference might be and how likely they are. The other is to set a value on the risk: to judge how harmful it will be.

The first is a task for natural science, but the second is not [Section 3.1]. As the Synthesis Report of AR4 states, "Determining what constitutes 'dangerous anthropogenic interference with the climate system' in relation to Article 2 of the UNFCCC involves value judgements". Judgements of value (valuations) are called for, not just here, but at almost every turn in decision making about climate change [3.2]. For example, setting a target for mitigation involves judging the value of losses to people's well-being in the future, and comparing it with the value of benefits enjoyed now. Choosing whether to site wind turbines on land or at sea requires a judgement of the value of landscape in comparison with the extra cost of marine turbines. To estimate the social cost of carbon is to value the harm that GHG emissions do [3.9.4].

Different values often conflict, and they are often hard to weigh against each other. Moreover, they often involve the conflicting interests of different people, and are subject to much debate and disagreement. Decision makers must therefore find ways to mediate among different interests and values, and also among differing viewpoints about values. [3.4, 3.5]

Social sciences and humanities can contribute to this process by improving our understanding of values in ways that are illustrated in the boxes contained in this summary. The sciences of human and social behaviour-among them psychology, political science, sociology, and non-normative branches of economics-investigate the values people have, how they change through time, how they can be influenced by political processes, and how the process of making decisions affects their acceptability. Other disciplines, including ethics (moral philosophy), decision theory, risk analysis, and the normative branch of economics, investigate, analyze, and clarify values themselves $[2.5,3.4,3.5,3.6]$. These disciplines offer practical ways of measuring some values and trading off conflicting interests. For example, the discipline of public health often measures health by means of 'disability-adjusted life years' [3.4.5]. Economics uses measures of social value that are generally based on monetary valuation but can take account of principles of distributive justice $[3.6,4.2,4.7,4.8]$. These normative disciplines also offer practical decision-making tools, such as expected utility theory, decision analysis, cost-benefit and cost-effectiveness analysis, and the structured use of expert judgment $[2.5,3.6,3.7$, 3.9].

There is a further element to decision making. People and countries have rights and owe duties towards each other. These are matters of justice, equity, or fairness. They fall within the subject matter of moral and political philosophy, jurisprudence, and economics. For example, some have argued that countries owe restitution for the harms that result from their past GHG emissions, and it has been debated, on jurisprudential and other grounds, whether restitution is owed only for harms that result from negligent or blameworthy GHG emissions. $[3.3,4.6]$ 
concentrations nor fully understand the harm it will impose on individuals, societies, and ecosystems. Article 2 requires that societies balance a variety of considerations - some rooted in the impacts of climate change itself and others in the potential costs of mitigation and adaptation. The difficulty of that task is compounded by the need to develop a consensus on fundamental issues such as the level of risk that societies are willing to accept and impose on others, strategies for sharing costs, and how to balance the numerous tradeoffs that arise because mitigation intersects with many other goals of societies. Such issues are inherently value-laden and involve different actors who have varied interests and disparate decision-making power.

The Working Group III (WGIII) contribution to the IPCC's Fifth Assessment Report (AR5) assesses literature on the scientific, technological, environmental, economic and social aspects of mitigation of climate change. It builds upon the WGIII contribution to the IPCC's Fourth Assessment Report (AR4), the Special Report on Renewable Energy Sources and Climate Change Mitigation (SRREN) and previous reports and incorporates subsequent new findings and research. Throughout, the focus is on the implications of its findings for policy, without being prescriptive about the particular policies that governments and other important participants in the policy process should adopt. In light of the IPCC's mandate, authors in WGIII were guided by several principles when assembling this assessment: (1) to be explicit about mitigation options, (2) to be explicit about their costs and about their risks and opportunities vis-à-vis other development priorities, (3) and to be explicit about the underlying criteria, concepts, and methods for evaluating alternative policies.

The remainder of this summary offers the main findings of this report. The degree of certainty in findings, as in the reports of all three IPCC Working Groups, is based on the author teams' evaluations of underlying scientific understanding and is expressed as a qualitative level of confidence (from very low to very high) and, when possible, probabilistically with a quantified likelihood (from exceptionally unlikely to virtually certain). Confidence in the validity of a finding is based on the type, amount, quality, and consistency of evidence (e.g., data, mechanistic understanding, theory, models, expert judgment) and the degree of agreement. Probabilistic estimates of quantified measures of uncertainty in a finding are based on statistical analysis of observations or model results, or both, and expert judgment. ${ }^{2}$ Where appropriate, find-

The following summary terms are used to describe the available evidence: limited, medium, or robust; and for the degree of agreement: low, medium, or high. A level of confidence is expressed using five qualifiers: very low, low, medium, high, and very high, and typeset in italics, e. g., medium confidence. For a given evidence and agreement statement, different confidence levels can be assigned, but increasing levels of evidence and degrees of agreement are correlated with increasing confidence. The following terms have been used to indicate the assessed likelihood of an outcome or a result: virtually certain $99-100 \%$ probability, very likely $90-100 \%$, likely $66-100 \%$, about as likely as not 33-66\%, unlikely 0-33\%, very unlikely $0-10 \%$, exceptionally unlikely $0-1 \%$. Additional terms (more likely than not $>50-100 \%$, and more unlikely than likely $0-<50 \%$ ) may also be used when appropriate. Assessed likelihood is typeset in italics, e. g., very likely. For more details, please refer to the Guidance Note for Lead Authors of the IPCC Fifth Assessment Report on Consistent Treatment of Uncertainties, available at http:// www.ipcc.ch/pdf/supporting-material/uncertainty-guidance-note.pdf. ings are also formulated as statements of fact without using uncertainty qualifiers. Within paragraphs of this summary, the confidence, evidence, and agreement terms given for a bolded finding apply to subsequent statements in the paragraph, unless additional terms are provided. References in [square brackets] indicate chapters, sections, figures, tables, and boxes where supporting evidence in the underlying report can be found.

This section continues with providing a framing of important concepts and methods that help to contextualize the findings presented in subsequent sections. Section TS.2 presents evidence on past trends in stocks and flows of GHGs and the factors that drive emissions at the global, regional, and sectoral scales including economic growth, technology, or population changes. Section TS.3.1 provides findings from studies that analyze the technological, economic, and institutional requirements of long-term mitigation scenarios. Section TS.3.2 provides details on mitigation measures and policies that are used within and across different economic sectors and human settlements. Section TS.4 summarizes insights on the interactions of mitigation policies between governance levels, economic sectors, and instrument types.

Climate change is a global commons problem that implies the need for international cooperation in tandem with local, national, and regional policies on many distinct matters. Because the GHG emissions of any agent (individual, company, country) affect every other agent, an effective outcome will not be achieved if individual agents advance their interests independently of others. International cooperation can contribute by defining and allocating rights and responsibilities with respect to the atmosphere [Sections 1.2.4, 3.1, $4.2,13.2 .1]$. Moreover, research and development (R\&D) in support of mitigation is a public good, which means that international cooperation can play a constructive role in the coordinated development and diffusion of technologies [1.4.4, 3.11, 13.9, 14.4.3]. This gives rise to separate needs for cooperation on R\&D, opening up of markets, and the creation of incentives to encourage private firms to develop and deploy new technologies and households to adopt them.

International cooperation on climate change involves ethical considerations, including equitable effort-sharing. Countries have contributed differently to the build-up of GHG in the atmosphere, have varying capacities to contribute to mitigation and adaptation, and have different levels of vulnerability to climate impacts. Many less developed countries are exposed to the greatest impacts but have contributed least to the problem. Engaging countries in effective international cooperation may require strategies for sharing the costs and benefits of mitigation in ways that are perceived to be equitable [4.2]. Evidence suggests that perceived fairness can influence the level of cooperation among individuals, and that finding may suggest that processes and outcomes seen as fair will lead to more international cooperation as well $[3.10,13.2 .2 .4]$. Analysis contained in the literature of moral and political philosophy can contribute to resolving ethical questions raised by climate change $[3.2,3.3,3.4]$. These questions include how much overall mitigation is needed to avoid 'dangerous interference with the climate system' (Box 


\section{Box TS.2 | Mitigation brings both market and non-market benefits to humanity}

The impacts of mitigation consist in the reduction or elimination of some of the effects of climate change. Mitigation may improve people's livelihood, their health, their access to food or clean water, the amenities of their lives, or the natural environment around them.

Mitigation can improve human well-being through both market and non-market effects. Market effects result from changes in market prices, in people's revenues or net income, or in the quality or availability of market commodities. Non-market effects result from changes in the quality or availability of non-marketed goods such as health, quality of life, culture, environmental quality, natural ecosystems, wildlife, and aesthetic values. Each impact of climate change can generate both market and non-market damages. For example, a heat wave in a rural area may cause heat stress for exposed farm labourers, dry up a wetland that serves as a refuge for migratory birds, or kill some crops and damage others. Avoiding these damages is a benefit of mitigation. [3.9]

Economists often use monetary units to value the damage done by climate change and the benefits of mitigation. The monetized value of a benefit to a person is the amount of income the person would be willing to sacrifice in order to get it, or alternatively the amount she would be willing to accept as adequate compensation for not getting it. The monetized value of a harm is the amount of income she would be willing to sacrifice in order to avoid it, or alternatively the amount she would be willing to accept as adequate compensation for suffering it. Economic measures seek to capture how strongly individuals care about one good or service relative to another, depending on their individual interests, outlook, and economic circumstances. [3.9]

Monetary units can be used in this way to measure costs and benefits that come at different times and to different people. But it cannot be presumed that a dollar to one person at one time can be treated as equivalent to a dollar to a different person or at a different time. Distributional weights may need to be applied between people [3.6.1], and discounting (see Box TS.10) may be appropriate between times. [3.6.2]
TS.1) [3.1], how the effort or cost of mitigating climate change should be shared among countries and between the present and future [3.3, $3.6,4.6]$, how to account for such factors as historical responsibility for GHG emissions $[3.3,4.6]$, and how to choose among alternative policies for mitigation and adaptation [3.4, 3.5, 3.6, 3.7]. Ethical issues of wellbeing, justice, fairness, and rights are all involved. Ethical analysis can identify the different ethical principles that underlie different viewpoints, and distinguish correct from incorrect ethical reasoning [3.3, 3.4].

Evaluation of mitigation options requires taking into account many different interests, perspectives, and challenges between and within societies. Mitigation engages many different agents, such as governments at different levels-regionally [14.1], nationally and locally [15.1], and through international agreements [13.1] —as well as households, firms, and other non-governmental actors. The interconnections between different levels of decision making and among different actors affect the many goals that become linked with climate policy. Indeed, in many countries the policies that have (or could have) the largest impact on emissions are motivated not solely by concerns surrounding climate change. Of particular importance are the interactions and perceived tensions between mitigation and development [4.1, 14.1]. Development involves many activities, such as enhancing access to modern energy services [7.9.1, 14.3.2, 16.8], the building of infrastructures [12.1], ensuring food security [11.1], and eradicating poverty [4.1]. Many of these activities can lead to higher emissions, if achieved by conventional means. Thus, the relationships between development and mitigation can lead to political and ethical conun- drums, especially for developing countries, when mitigation is seen as exacerbating urgent development challenges and adversely affecting the current well-being of their populations [4.1]. These conundrums are examined throughout this report, including in special boxes highlighting the concerns of developing countries.

Economic evaluation can be useful for policy design and be given a foundation in ethics, provided appropriate distributional weights are applied. While the limitations of economics are widely documented $[2.4,3.5]$, economics nevertheless provides useful tools for assessing the pros and cons of mitigation and adaptation options. Practical tools that can contribute to decision making include cost-benefit analysis, cost-effectiveness analysis, multi-criteria analysis, expected utility theory, and methods of decision analysis [2.5, 3.7.2]. Economic valuation (see Box TS.2) can be given a foundation in ethics, provided distributional weights are applied that take proper account of the difference in the value of money to rich and poor people [3.6]. Few empirical applications of economic valuation to climate change have been well-founded in this respect [3.6.1]. The literature provides significant guidance on the social discount rate for consumption (see Box TS.10), which is in effect inter-temporal distributional weighting. It suggests that the social discount rate depends in a well-defined way primarily on the anticipated growth in per capita income and inequality aversion [3.6.2].

Most climate policies intersect with other societal goals, either positively or negatively, creating the possibility of 'co-benefits' 


\section{Box TS.3 | Deliberative and intuitive thinking are inputs to effective risk management}

When people-from individual voters to key decision makers in firms to senior government policymakers - make choices that involve risk and uncertainty, they rely on deliberative as well intuitive thought processes. Deliberative thinking is characterized by the use of a wide range of formal methods to evaluate alternative choices when probabilities are difficult to specify and/or outcomes are uncertain. They can enable decision makers to compare choices in a systematic manner by taking into account both short and long-term consequences. A strength of these methods is that they help avoid some of the well-known pitfalls of intuitive thinking, such as the tendency of decision makers to favour the status quo. A weakness of these deliberative decision aids is that they are often highly complex and require considerable time and attention.

Most analytically based literature, including reports such as this one, is based on the assumption that individuals undertake deliberative and systematic analyses in comparing options. However, when making mitigation and adaptation choices, people are also likely to engage in intuitive thinking. This kind of thinking has the advantage of requiring less extensive analysis than deliberative thinking. However, relying on one's intuition may not lead one to characterize problems accurately when there is limited past experience. Climate change is a policy challenge in this regard since it involves large numbers of complex actions by many diverse actors, each with their own values, goals, and objectives. Individuals are likely to exhibit well-known patterns of intuitive thinking such as making choices related to risk and uncertainty on the basis of emotional reactions and the use of simplified rules that have been acquired by personal experience. Other tendencies include misjudging probabilities, focusing on short time horizons, and utilizing rules of thumb that selectively attend to subsets of goals and objectives. [2.4]

By recognizing that both deliberative and intuitive modes of decision making are prevalent in the real world, risk management programmes can be developed that achieve their desired impacts. For example, alternative frameworks that do not depend on precise specification of probabilities and outcomes can be considered in designing mitigation and adaptation strategies for climate change. $[2.4,2.5,2.6]$ or 'adverse side-effects'. Since the publication of AR4, a substantial body of literature has emerged looking at how countries that engage in mitigation also address other goals, such as local environmental protection or energy security, as a 'co-benefit' and conversely [1.2.1, 6.6.1, 4.8]. This multi-objective perspective is important because it helps to identify areas where political, administrative, stakeholder, and other support for policies that advance multiple goals will be robust. Moreover, in many societies the presence of multiple objectives may make it easier for governments to sustain the political support needed for mitigation [15.2.3]. Measuring the net effect on social welfare (see Box TS.11) requires examining the interaction between climate policies and pre-existing other policies $[3.6 .3,6.3 .6 .5]$.

Mitigation efforts generate tradeoffs and synergies with other societal goals that can be evaluated in a sustainable development framework. The many diverse goals that societies value are often called 'sustainable development'. A comprehensive assessment of climate policy therefore involves going beyond a narrow focus on distinct mitigation and adaptation options and their specific co-benefits and adverse side-effects. Instead it entails incorporating climate issues into the design of comprehensive strategies for equitable and sustainable development at regional, national, and local levels [4.2, 4.5]. Maintaining and advancing human well-being, in particular overcoming poverty and reducing inequalities in living standards, while avoiding unsustainable patterns of consumption and production, are fundamental aspects of equitable and sustainable development [4.4, $4.6,4.8]$. Because these aspects are deeply rooted in how societies for- mulate and implement economic and social policies generally, they are critical to the adoption of effective climate policy.

Variations in goals reflect, in part, the fact that humans perceive risks and opportunities differently. Individuals make their decisions based on different goals and objectives and use a variety of different methods in making choices between alternative options. These choices and their outcomes affect the ability of different societies to cooperate and coordinate. Some groups put greater emphasis on near-term economic development and mitigation costs, while others focus more on the longer-term ramifications of climate change for prosperity. Some are highly risk averse while others are more tolerant of dangers. Some have more resources to adapt to climate change and others have fewer. Some focus on possible catastrophic events while others ignore extreme events as implausible. Some will be relative winners, and some relative losers from particular climate changes. Some have more political power to articulate their preferences and secure their interests and others have less. Since AR4, awareness has grown that such considerations - long the domain of psychology, behavioural economics, political economy, and other disciplines-need to be taken into account in assessing climate policy (see Box TS.3). In addition to the different perceptions of climate change and its risks, a variety of norms can also affect what humans view as acceptable behaviour. Awareness has grown about how such norms spread through social networks and ultimately affect activities, behaviours and lifestyles, and thus development pathways, which can have profound impacts on GHG emissions and mitigation policy. $[1.4 .2,2.4,3.8,3.10,4.3]$ 


\section{Box TS.4 |'Fat tails': unlikely vs. likely outcomes in understanding the value of mitigation}

What has become known as the 'fat-tails' problem relates to uncertainty in the climate system and its implications for mitigation and adaptation policies. By assessing the chain of structural uncertainties that affect the climate system, the resulting compound probability distribution of possible economic damage may have a fat right tail. That means that the probability of damage does not decline with increasing temperature as quickly as the consequences rise.

The significance of fat tails can be illustrated for the distribution of temperature that will result from a doubling of atmospheric carbon dioxide $\left(\mathrm{CO}_{2}\right)$ (climate sensitivity). IPCC Working Group I (WGI) estimates may be used to calibrate two possible distributions, one fat-tailed and one thin-tailed, that each have a median temperature change of $3^{\circ} \mathrm{C}$ and a $15 \%$ probability of a temperature change in excess of $4.5^{\circ} \mathrm{C}$. Although the probability of exceeding $4.5^{\circ} \mathrm{C}$ is the same for both distributions, likelihood drops off much more slowly with increasing temperature for the fat-tailed compared to the thin-tailed distribution. For example, the probability of temperatures in excess of $8^{\circ} \mathrm{C}$ is nearly ten times greater with the chosen fat-tailed distribution than with the thin-tailed distribution. If temperature changes are characterized by a fat tailed distribution, and events with large impact may occur at higher temperatures, then tail events can dominate the computation of expected damages from climate change.

In developing mitigation and adaptation policies, there is value in recognizing the higher likelihood of tail events and their consequences. In fact, the nature of the probability distribution of temperature change can profoundly change how climate policy is framed and structured. Specifically, fatter tails increase the importance of tail events (such as $8^{\circ} \mathrm{C}$ warming). While research attention and much policy discussion have focused on the most likely outcomes, it may be that those in the tail of the probability distribution are more important to consider. [2.5, 3.9.2]
Effective climate policy involves building institutions and capacity for governance. While there is strong evidence that a transition to a sustainable and equitable path is technically feasible, charting an effective and viable course for climate change mitigation is not merely a technical exercise. It will involve myriad and sequential decisions among states and civil society actors. Such a process benefits from the education and empowerment of diverse actors to participate in systems of decision making that are designed and implemented with procedural equity as a deliberate objective. This applies at the national as well as international levels, where effective governance relating to global common resources, in particular, is not yet mature. Any given approach has potential winners and losers. The political feasibility of that approach will depend strongly on the distribution of power, resources, and decision-making authority among the potential winners and losers. In a world characterized by profound disparities, procedurally equitable systems of engagement, decision making and governance may help enable a polity to come to equitable solutions to the sustainable development challenge. [4.3]

Effective risk management of climate change involves considering uncertainties in possible physical impacts as well as human and social responses. Climate change mitigation and adaptation is a risk management challenge that involves many different decisionmaking levels and policy choices that interact in complex and often unpredictable ways. Risks and uncertainties arise in natural, social, and technological systems. As Box TS.3 explains, effective risk management strategies not only consider people's values, and their intuitive decision processes but utilize formal models and decision aids for systematically addressing issues of risk and uncertainty $[2.4,2.5]$. Research on other such complex and uncertainty-laden policy domains suggest the importance of adopting policies and measures that are robust across a variety of criteria and possible outcomes [2.5]. As detailed in Box TS.4, a special challenge arises with the growing evidence that climate change may result in extreme impacts whose trigger points and outcomes are shrouded in high levels of uncertainty [2.5, 3.9.2]. A risk management strategy for climate change will require integrating responses in mitigation with different time horizons, adaptation to an array of climate impacts, and even possible emergency responses such as 'geoengineering' in the face of extreme climate impacts [1.4.2, $3.3 .7,6.9,13.4 .4]$. In the face of potential extreme impacts, the ability to quickly offset warming could help limit some of the most extreme climate impacts although deploying these geoengineering systems could create many other risks (see Section TS.3.1.3). One of the central challenges in developing a risk management strategy is to have it adaptive to new information and different governing institutions [2.5].

\section{TS.2 Trends in stocks and flows of greenhouse gases and their drivers}

This section summarizes historical GHG emissions trends and their underlying drivers. As in most of the underlying literature, all aggregate $\mathrm{GHG}$ emissions estimates are converted to $\mathrm{CO}_{2}$-equivalents based on Global Warming Potentials with a 100-year time horizon (GWP ${ }_{100}$ ) (Box TS.5). The majority of changes in GHG emissions trends that are observed in this section are related to changes in drivers such as eco- 
Total Annual Anthropogenic GHG Emissions by Groups of Gases 1970-2010

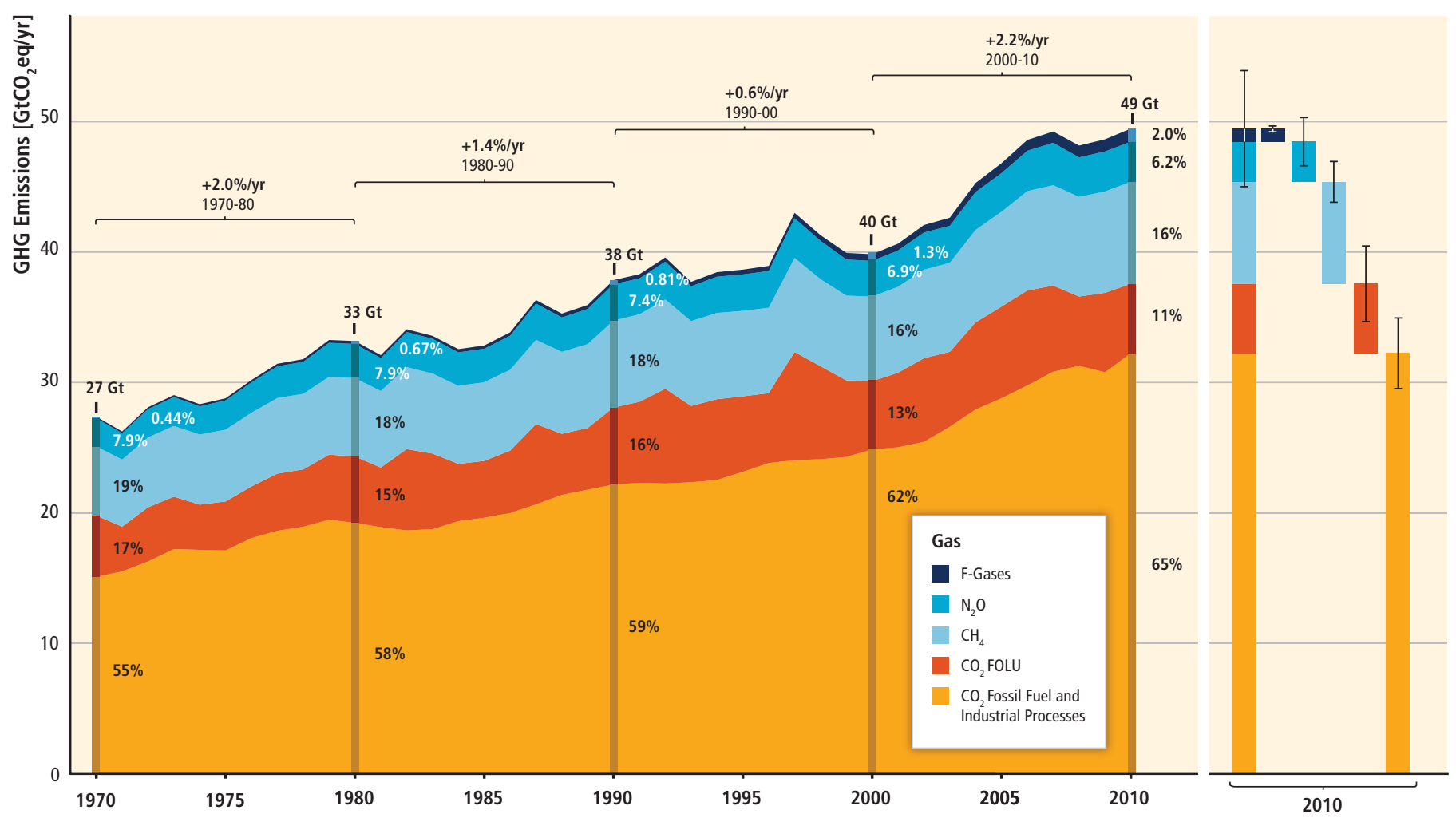

Figure TS.1| Total annual anthropogenic GHG emissions $\left(\mathrm{GtCO}_{2} \mathrm{eq} / \mathrm{yr}\right)$ by groups of gases 1970-2010: carbon dioxide $\left(\mathrm{CO}_{2}\right)$ from fossil fuel combustion and industrial processes; $\mathrm{CO}_{2}$ from Forestry and Other Land Use $(\mathrm{FOLU})$; methane $\left(\mathrm{CH}_{4}\right)$; nitrous oxide $\left(\mathrm{N}_{2} \mathrm{O}\right)$; fluorinated gases ${ }^{5}$ covered under the Kyoto Protocol (F-gases). At the right side of the figure, GHG emissions in 2010 are shown again broken down into these components with the associated uncertainties ( $90 \%$ confidence interval) indicated by the error bars. Total anthropogenic GHG emissions uncertainties are derived from the individual gas estimates as described in Chapter 5 [5.2.3.6]. Emissions are converted into $\mathrm{CO}_{2}$-equivalents based on Global Warming Potentials with a 100-year time horizon $\left(\mathrm{GWP}_{100}\right)$ from the IPCC Second Assessment Report (SAR). The emissions data from FOLU represents land-based $\mathrm{CO}_{2}$ emissions from forest and peat fires and decay that approximate to the net $\mathrm{CO}_{2}$ flux from FOLU as described in Chapter 11 of this report. Average annual GHG emissions growth rates for the four decades are highlighted with the brackets. The average annual growth rate from 1970 to 2000 is $1.3 \%$. [Figure 1.3]

nomic growth, technological change, human behaviour, or population growth. But there are also some smaller changes in GHG emissions estimates that are due to refinements in measurement concepts and methods that have happened since AR4. There is a growing body of literature on uncertainties in global GHG emissions data sets. This section tries to make these uncertainties explicit and reports variations in estimates across global data sets wherever possible.

\section{TS.2.1 Greenhouse gas emission trends}

Total anthropogenic GHG emissions have risen more rapidly from 2000 to 2010 than in the previous three decades (high confidence). Total anthropogenic GHG emissions were the highest in human history from 2000 to 2010 and reached $49( \pm 4.5)$ gigatonnes $\mathrm{CO}_{2}$-equivalents per year ( $\mathrm{GtCO}_{2} \mathrm{eq} / \mathrm{yr}$ ) in 2010. ${ }^{3}$ Current trends are at the high end of levels that had been projected for this last decade. GHG emissions growth has occurred despite the presence of a wide array of multilateral institutions as well as national policies aimed at mitigation. From 2000 to 2010, GHG emissions grew on average by $1.0 \mathrm{GtCO}_{2} \mathrm{eq}(2.2 \%)$ per year compared to $0.4 \mathrm{GtCO}_{2} \mathrm{eq}(1.3 \%)$ per year over the entire period from 1970 to 2000 (Figure TS.1). The global economic crisis 2007/2008 has only temporarily reduced GHG emissions. $[1.3,5.2$, 13.3, 15.2.2, Figure 15.1] 
Total Anthropogenic $\mathrm{CO}_{2}$ Emissions from Fossil Fuel Combustion, Flaring, Cement, as well as Forestry and Other Land Use (FOLU) by Region between 1750 and 2010

a)

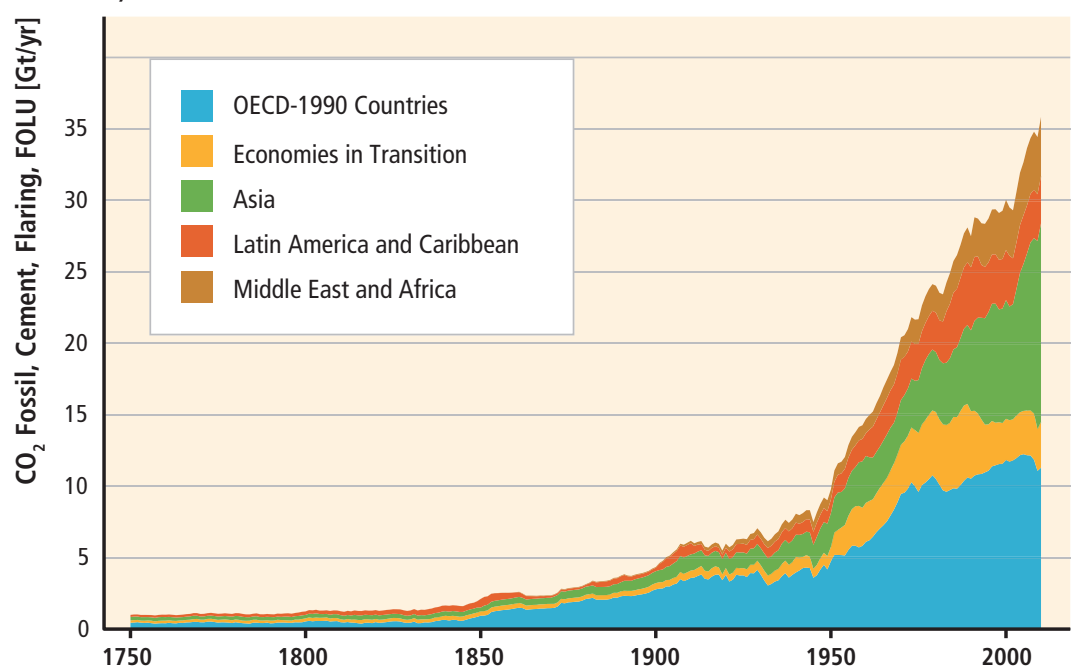

c)

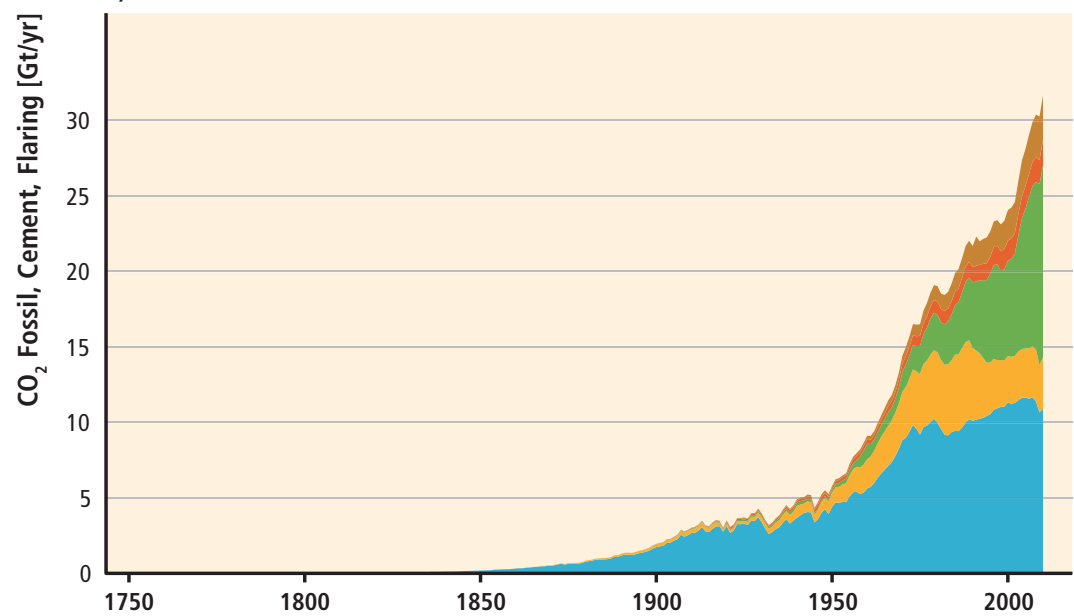

e)

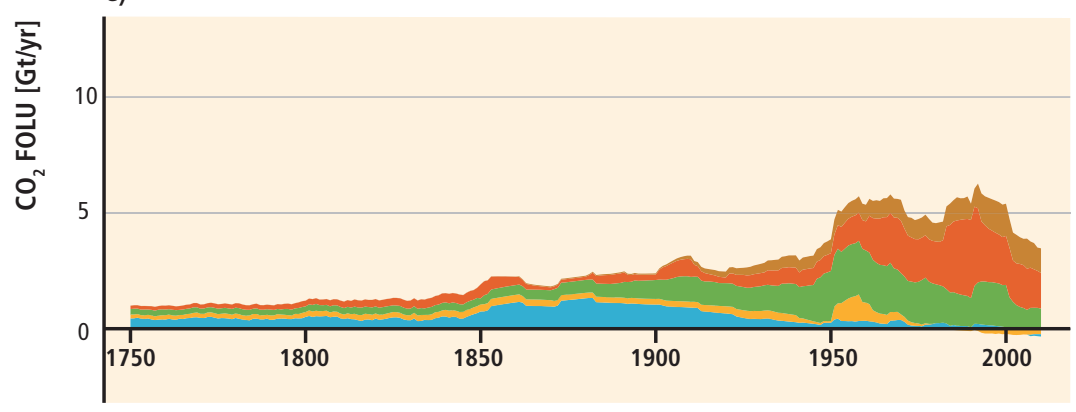

b)

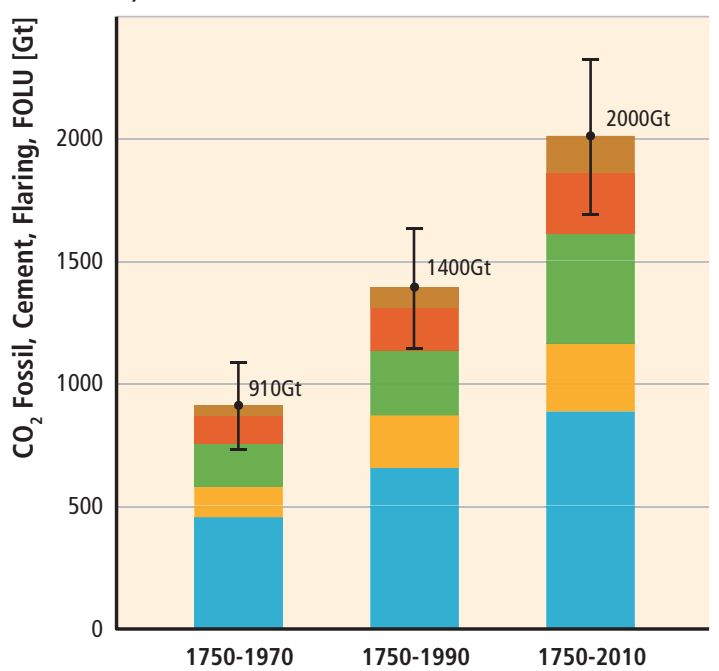

d)

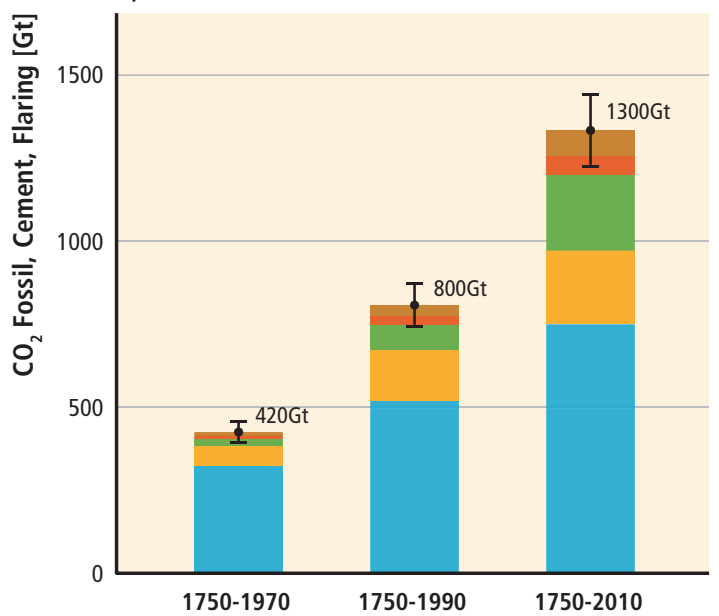

f)

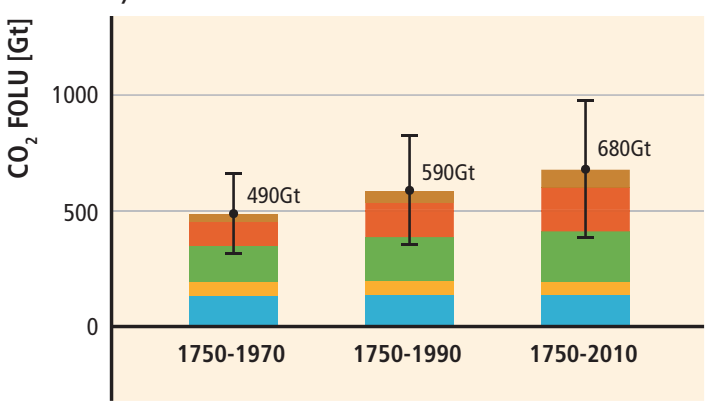

Figure TS.2 | Historical anthropogenic $\mathrm{CO}_{2}$ emissions from fossil fuel combustion, flaring, cement, and Forestry and Other Land Use (FOLU) ${ }^{4}$ in five major world regions: OECD1990 (blue); Economies in Transition (yellow); Asia (green); Latin America and Caribbean (red); Middle East and Africa (brown). Emissions are reported in gigatonnes of $\mathrm{CO}_{2}$ per year $\left(\mathrm{GtCO}_{2} / \mathrm{yr}\right)$. Left panels show regional $\mathrm{CO}_{2}$ emissions 1750-2010 from: (a) the sum of all $\mathrm{CO}_{2}$ sources $(\mathrm{c}+\mathrm{e})$; (c) fossil fuel combustion, flaring, and cement; and (e) FOLU. The right panels report regional contributions to cumulative $\mathrm{CO}_{2}$ emissions over selected time periods from: (b) the sum of all $\mathrm{CO}_{2}$ sources ( $\mathrm{d}+\mathrm{f}$ ); (d) fossil fuel combustion, flaring and cement; and (f) FOLU. Error bars on panels (b), (d) and (f) give an indication of the uncertainty range ( $90 \%$ confidence interval). See Annex II.2.2 for definitions of regions. [Figure 5.3] 


\section{Greenhouse Gas Emissions by Economic Sectors}

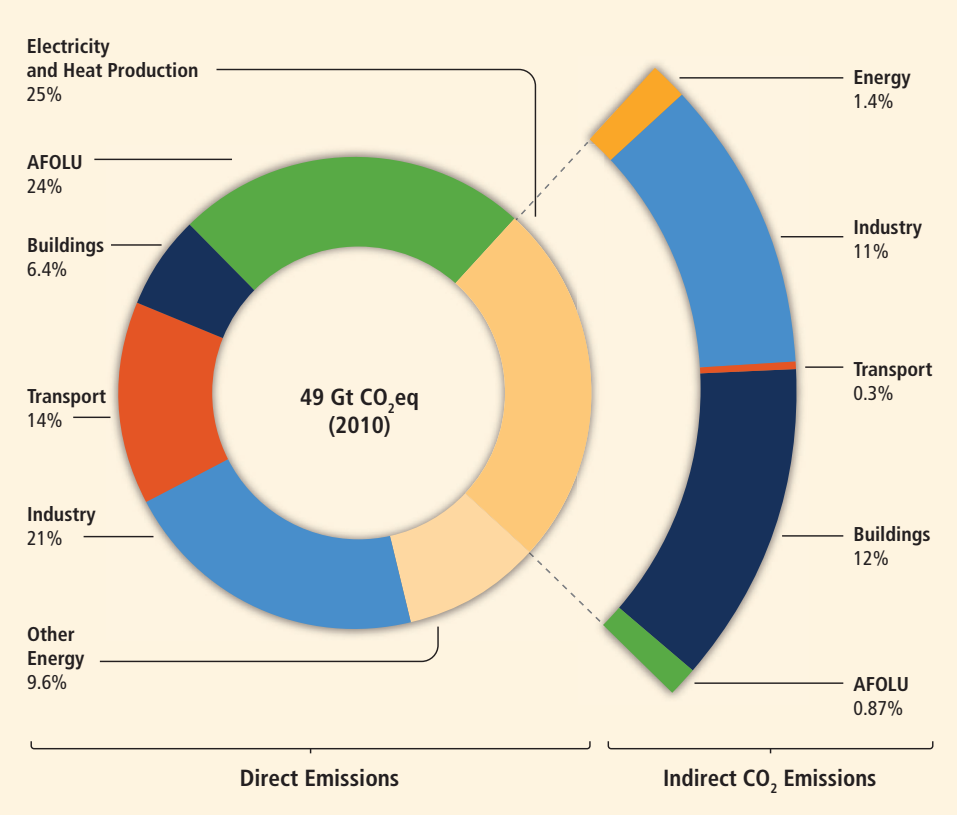

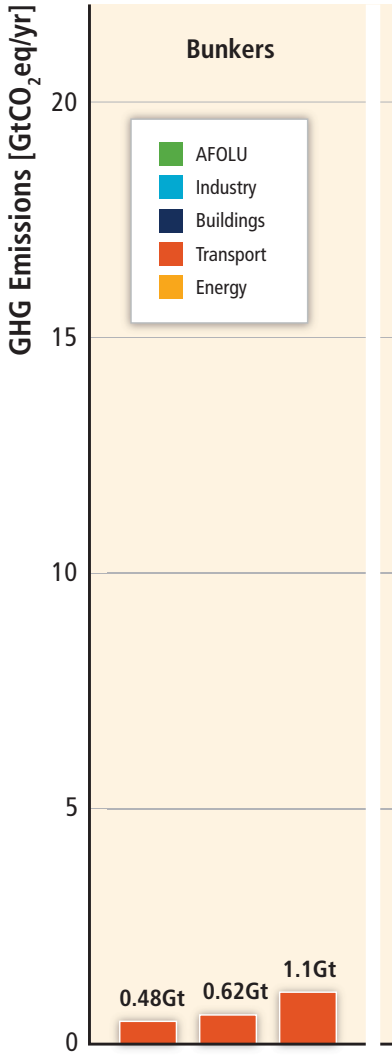

$19701990 \quad 2010$

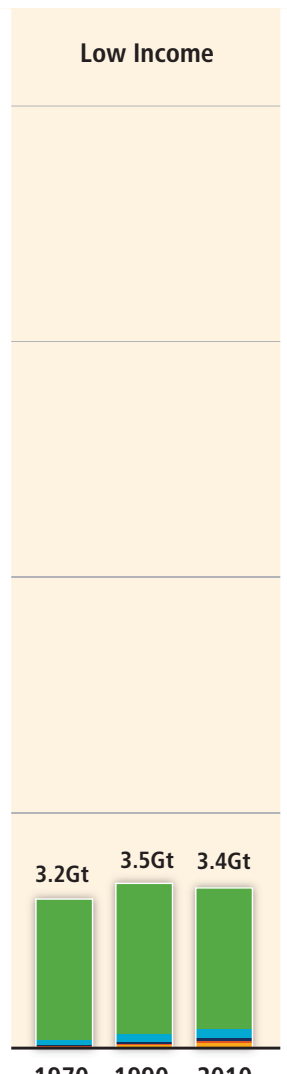

197019902010

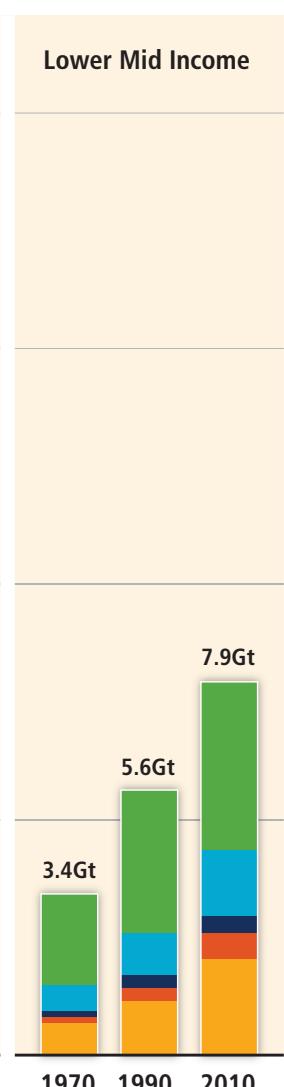

197019902010

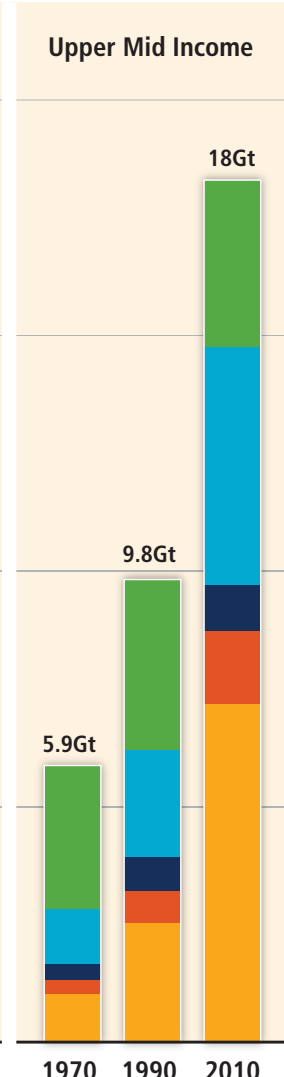

197019902010

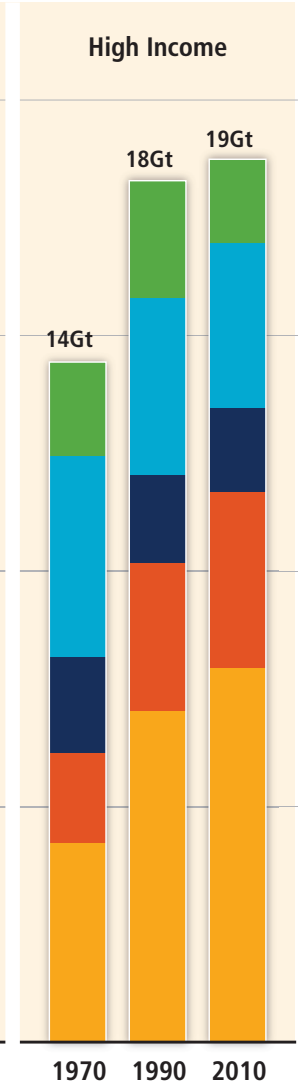

Figure TS.3 | Total anthropogenic GHG emissions ( $\mathrm{GtCO}_{2}$ eq/yr) by economic sectors and country income groups. Upper panel: Circle shows direct GHG emission shares (in \% of total anthropogenic GHG emissions) of five major economic sectors in 2010. Pull-out shows how indirect $\mathrm{CO}_{2}$ emission shares (in \% of total anthropogenic GHG emissions) from electricity and heat production are attributed to sectors of final energy use. 'Other Energy' refers to all GHG emission sources in the energy sector other than electricity and heat production. Lower panel: Total anthropogenic GHG emissions in 1970, 1990 and 2010 by five major economic sectors and country income groups. 'Bunkers' refer to GHG emissions from international transportation and thus are not, under current accounting systems, allocated to any particular nation's territory. The emissions data from Agriculture, Forestry and Other Land Use (AFOLU) includes land-based $\mathrm{CO}_{2}$ emissions from forest and peat fires and decay that approximate to the net $\mathrm{CO}_{2}$ flux from the Forestry and Other Land Use (FOLU) sub-sector as described in Chapter 11 of this report. Emissions are converted into $\mathrm{CO}_{2}$-equivalents based on Global Warming Potentials with a 100-year time horizon (GWP ${ }_{100}$ ) from the IPCC Second Assessment Report (SAR). Assignment of countries to income groups is based on the World Bank income classification in 2013. For details see Annex II.2.3. Sector definitions are provided in Annex II.9.1. [Figure 1.3, Figure 1.6] 

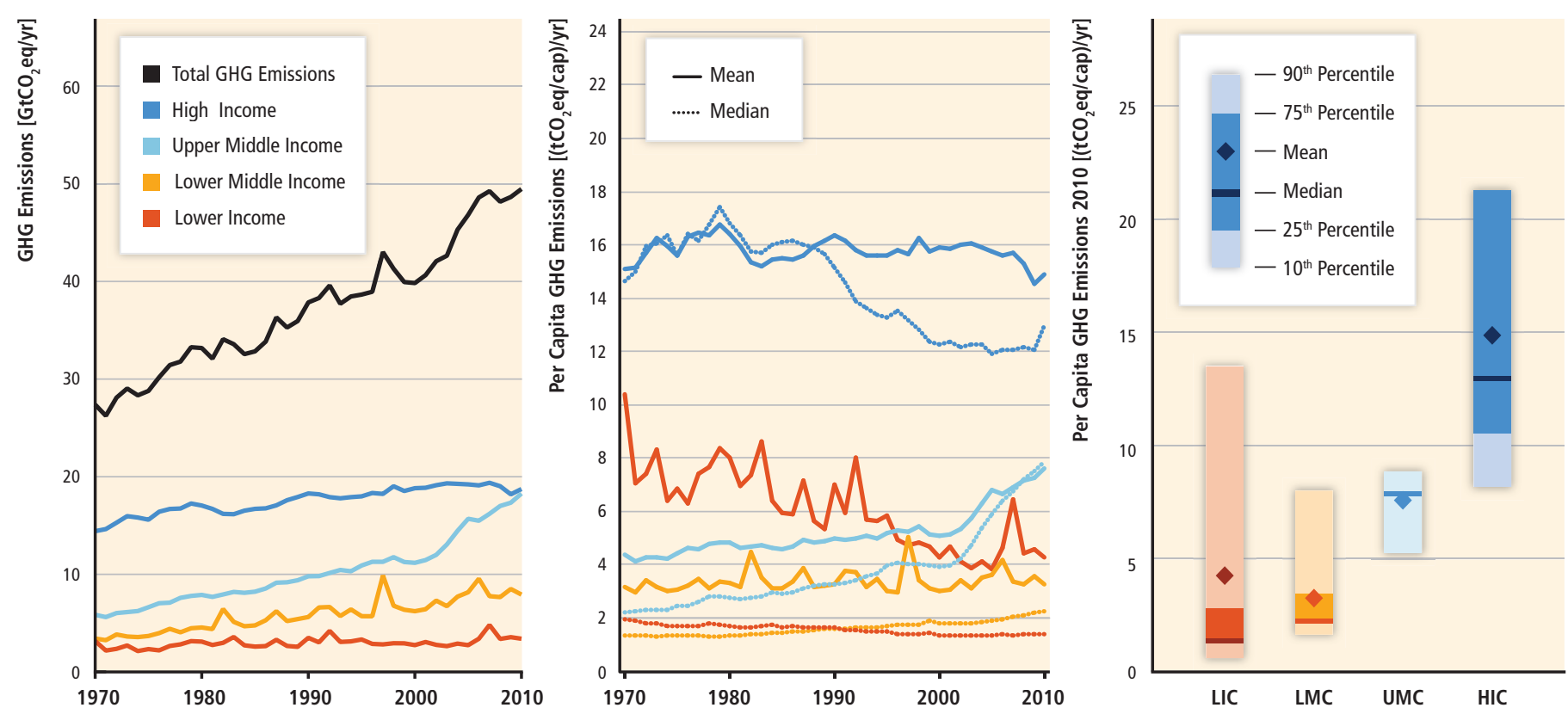

Figure TS.4| Trends in GHG emissions by country income groups. Left panel: Total annual anthropogenic GHG emissions from 1970 to 2010 (GtCO 2 eq/yr). Middle panel: Trends in annual per capita mean and median GHG emissions from 1970 to 2010 ( $\mathrm{tCO}$ eq/cap/yr). Right panel: Distribution of annual per capita $\mathrm{GHG}$ emissions in 2010 of countries within each country income group ( $\left.\mathrm{tCO}_{2} / \mathrm{cap} / \mathrm{yr}\right)$. Mean values show the $\mathrm{GHG}$ emissions levels weighed by population. Median values describe GHG emissions levels per capita of the country at the 50th percentile of the distribution within each country income group. Emissions are converted into $\mathrm{CO}_{2}$-equivalents based on Global Warming Potentials with a 100 year time horizon (GWP $\left.{ }_{100}\right)$ from the IPCC Second Assessment Report (SAR). Assignment of countries to country income groups is based on the World Bank income classification in 2013. For details see Annex II.2.3. [Figures 1.4, 1.8]

$\mathrm{CO}_{2}$ emissions from fossil fuel combustion and industrial processes contributed about $78 \%$ to the total GHG emissions increase from 1970 to 2010, with similar percentage contribution for the period 2000-2010 (high confidence). Fossil fuel-related $\mathrm{CO}_{2}$ emissions reached $32( \pm 2.7) \mathrm{GtCO}_{2} / \mathrm{yr}$ in 2010 and grew further by about $3 \%$ between 2010 and 2011 and by about 1-2\% between 2011 and 2012. Since AR4, the shares of the major groups of GHG emissions have remained stable. Of the $49( \pm 4.5) \mathrm{GtCO}_{2}$ eq/yr in total anthropogenic GHG emissions in 2010, $\mathrm{CO}_{2}$ remains the major GHG accounting for $76 \%\left(38 \pm 3.8 \mathrm{GtCO}_{2} \mathrm{eq} / \mathrm{yr}\right)$ of total anthropogenic GHG emissions. $16 \%\left(7.8 \pm 1.6 \mathrm{GtCO}_{2} \mathrm{eq} / \mathrm{yr}\right)$ come from methane $\left(\mathrm{CH}_{4}\right)$, $6.2 \%\left(3.1 \pm 1.9 \mathrm{GtCO}_{2} \mathrm{eq} / \mathrm{yr}\right)$ from nitrous oxide $\left(\mathrm{N}_{2} \mathrm{O}\right)$, and $2.0 \%$ $\left(1.0 \pm 0.2 \mathrm{GtCO}_{2} \mathrm{eq} / \mathrm{yr}\right)$ from fluorinated gases (Figure TS.1). ${ }^{5}$ Using the most recent GWP ${ }_{100}$ values from the AR5 [WGI 8.7] global GHG emissions totals would be slightly higher $\left(52 \mathrm{GtCO}_{2} \mathrm{eq} / \mathrm{yr}\right)$ and non- $\mathrm{CO}_{2}$ emission shares would be $20 \%$ for $\mathrm{CH}_{4}, 5.0 \%$ for $\mathrm{N}_{2} \mathrm{O}$ and $2.2 \%$ for F-gases. Emission shares are sensitive to the choice of emission metric and time horizon, but this has a small influence on global, long-term trends. If a shorter, 20-year time horizon were used, then the share of $\mathrm{CO}_{2}$ would decline to just over $50 \%$ of total anthropogenic GHG emissions and short-lived gases would rise in relative importance. As detailed in Box TS.5, the choice of emission metric and time horizon involves explicit or implicit value judgements and depends on the purpose of the analysis. [1.2, 3.9, 5.2]
Over the last four decades total cumulative $\mathrm{CO}_{2}$ emissions have increased by a factor of 2 from about $910 \mathrm{GtCO}_{2}$ for the period 1750-1970 to about $2000 \mathrm{GtCO}_{2}$ for 1750-2010 (high confidence). In 1970, the cumulative $\mathrm{CO}_{2}$ emissions from fossil fuel combustion, cement production and flaring since 1750 was $420( \pm 35) \mathrm{GtCO}_{2}$; in 2010 that cumulative total had tripled to $1300( \pm 110) \mathrm{GtCO}_{2}$ (Figure TS.2). Cumulative $\mathrm{CO}_{2}$ emissions associated with $\mathrm{FOLU}^{4}$ since 1750 increased from about $490( \pm 180) \mathrm{GtCO}_{2}$ in 1970 to approximately 680 $( \pm 300) \mathrm{GtCO}_{2}$ in 2010. [5.2]

Regional patterns of GHG emissions are shifting along with changes in the world economy (high confidence). Since 2000, GHG emissions have been growing in all sectors, except Agriculture, Forestry and Other Land Use (AFOLU) ${ }^{4}$ where positive and negative emission changes are reported across different databases and uncertainties in the data are high. More than $75 \%$ of the $10 \mathrm{Gt}$ increase in annual GHG emissions between 2000 and 2010 was emitted in the energy supply $(47 \%)$ and industry $(30 \%)$ sectors (see Annex II.9.I for sector definitions). $5.9 \mathrm{GtCO}_{2}$ eq of this sectoral increase occurred in upper-middle income countries, ${ }^{6}$ where the most rapid economic development and infrastructure expansion has taken place. GHG emissions growth in the other sectors has been more modest in absolute $\left(0.3-1.1 \mathrm{Gt} \mathrm{CO}_{2} \mathrm{eq}\right)$ as well as in relative terms $(3 \%-11 \%)$. [1.3, 5.3, Figure 5.18]

\footnotetext{
When countries are assigned to income groups in this summary, the World Bank income classification for 2013 is used. For details see Annex II.2.3.
} 


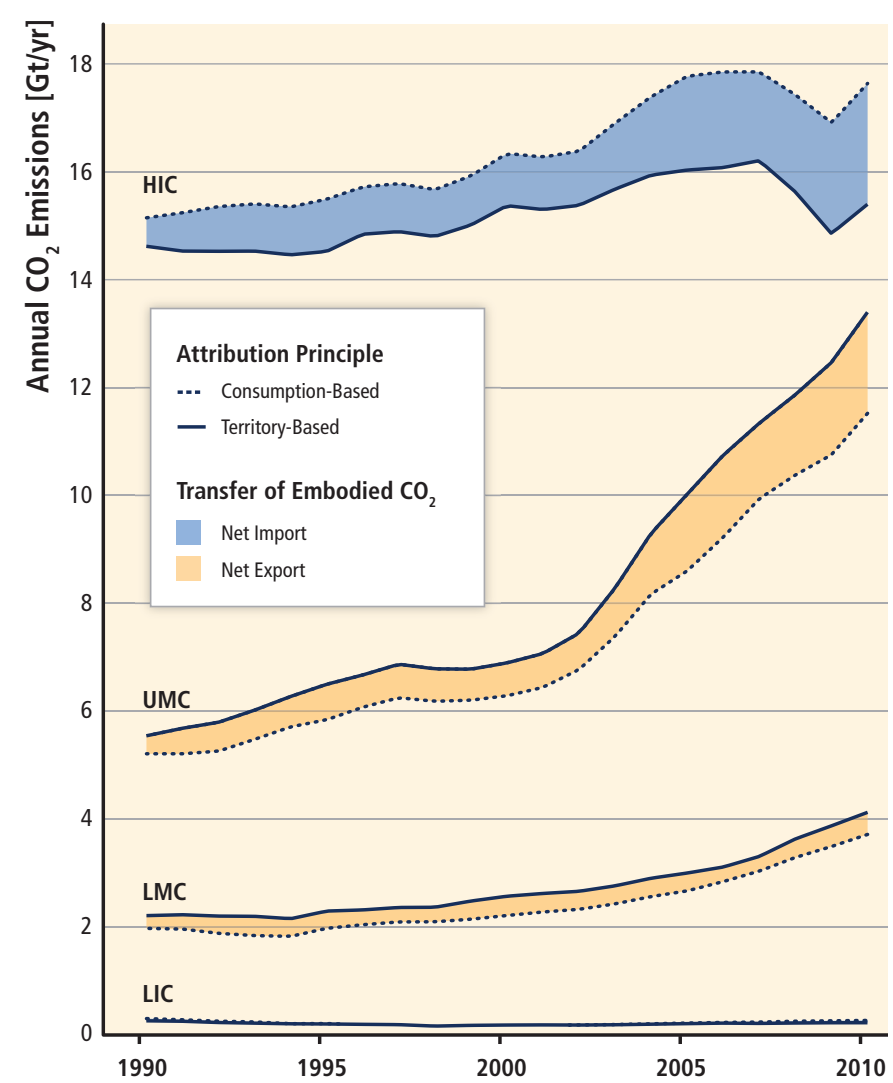

Figure TS.5 | Total annual $\mathrm{CO}_{2}$ emissions $\left(\mathrm{GtCO}_{2} / \mathrm{yr}\right)$ from fossil fuel combustion for country income groups attributed on the basis of territory (solid line) and final consumption (dotted line). The shaded areas are the net $\mathrm{CO}_{2}$ trade balances (differences) between each of the four country income groups and the rest of the world. Blue shading indicates that the country income group is a net importer of embodied $\mathrm{CO}_{2}$ emissions, leading to consumption-based emission estimates that are higher than traditional territorial emission estimates. Orange indicates the reverse situation-the country income group is a net exporter of embodied $\mathrm{CO}_{2}$ emissions. Assignment of countries to country income groups is based on the World Bank income classification in 2013. For details see Annex II.2.3. [Figure 1.5]

Current GHG emission levels are dominated by contributions from the energy supply, AFOLU, and industry sectors; industry and buildings gain considerably in importance if indirect emissions are accounted for (robust evidence, high agreement). Of the $49( \pm 4.5) \mathrm{GtCO}_{2}$ eq emissions in $2010,35 \%\left(17 \mathrm{GtCO}_{2}\right.$ eq $)$ of GHG emissions were released in the energy supply sector, $24 \%$ (12 $\mathrm{GtCO}_{2}$ eq, net emissions) in AFOLU, $21 \%\left(10 \mathrm{GtCO}_{2}\right.$ eq) in industry, $14 \%\left(7.0 \mathrm{GtCO}_{2} \mathrm{eq}\right)$ in transport, and $6.4 \%\left(3.2 \mathrm{GtCO}_{2} \mathrm{eq}\right)$ in buildings. When indirect emissions from electricity and heat production are assigned to sectors of final energy use, the shares of the industry and buildings sectors in global GHG emissions grow to $31 \%$ and $19 \%,{ }^{3}$ respectively (Figure TS.3 upper panel). $[1.3,7.3,8.2,9.2$, $10.3,11.2]$

Per capita GHG emissions in $\mathbf{2 0 1 0}$ are highly unequal (high confidence). In 2010, median per capita GHG emissions (1.4 $\mathrm{tCO}_{2} \mathrm{eq} / \mathrm{cap} / \mathrm{yr}$ ) for the group of low-income countries are around nine times lower than median per capita GHG emissions ( $13 \mathrm{tCO}_{2}$ eq/cap/yr) of highincome countries (Figure TS.4). ${ }^{6}$ For low-income countries, the largest part of GHG emissions comes from AFOLU; for high-income countries, GHG emissions are dominated by sources related to energy supply and industry (Figure TS.3 lower panel). There are substantial variations in per capita GHG emissions within country income groups with emissions at the 90th percentile level more than double those at the 10th percentile level. Median per capita emissions better represent the typical country within a country income group comprised of heterogeneous members than mean per capita emissions. Mean per capita GHG emissions are different from median mainly in low-income countries as individual low-income countries have high per capita emissions due to large $\mathrm{CO}_{2}$ emissions from land-use change (Figure TS.4, right panel). $[1.3,5.2,5.3]$

A growing share of total anthropogenic $\mathrm{CO}_{2}$ emissions is released in the manufacture of products that are traded across international borders (medium evidence, high agreement). Since AR4, several data sets have quantified the difference between traditional 'territorial' and 'consumption-based' emission estimates that assign all emission released in the global production of goods and services to the country of final consumption (Figure TS.5). A growing share of $\mathrm{CO}_{2}$ emissions from fossil fuel combustion in middle income countries is released in the production of goods and services exported, notably from upper middle income countries to high income countries. Total annual industrial $\mathrm{CO}_{2}$ emissions from the non-Annex I group now exceed those of the Annex I group using territorial and consumptionbased accounting methods, but per-capita emissions are still markedly higher in the Annex I group. [1.3, 5.3]

Regardless of the perspective taken, the largest share of anthropogenic $\mathrm{CO}_{2}$ emissions is emitted by a small number of countries (high confidence). In 2010, 10 countries accounted for about $70 \%$ of $\mathrm{CO}_{2}$ emissions from fossil fuel combustion and industrial processes. A similarly small number of countries emit the largest share of consumption-based $\mathrm{CO}_{2}$ emissions as well as cumulative $\mathrm{CO}_{2}$ emissions going back to 1750 . [1.3]

The upward trend in global fossil fuel related $\mathrm{CO}_{2}$ emissions is robust across databases and despite uncertainties (high confidence). Global $\mathrm{CO}_{2}$ emissions from fossil fuel combustion are known within $8 \%$ uncertainty. $\mathrm{CO}_{2}$ emissions related to FOLU have very large uncertainties attached in the order of $50 \%$. Uncertainty for global emissions of methane $\left(\mathrm{CH}_{4}\right)$, nitrous oxide $\left(\mathrm{N}_{2} \mathrm{O}\right)$, and the fluorinated gases has been estimated as $20 \%, 60 \%$, and $20 \%$. Combining these values yields an illustrative total global GHG uncertainty estimate of about $10 \%$ (Figure TS.1). Uncertainties can increase at finer spatial scales and for specific sectors. Attributing GHG emissions to the country of final consumption increases uncertainties, but literature on this topic is just emerging. GHG emissions estimates in the AR4 were 5-10\% higher than the estimates reported here, but lie within the estimated uncertainty range. ${ }^{3}[5.2]$ 


\section{Box TS.5 | Emissions metrics depend on value judgements and contain wide uncertainties}

Emission metrics provide 'exchange rates' for measuring the contributions of different GHGs to climate change. Such exchange rates serve a variety of purposes, including apportioning mitigation efforts among several gases and aggregating emissions of a variety of GHGs. However, there is no metric that is both conceptually correct and practical to implement. Because of this, the choice of the appropriate metric depends on the application or policy at issue. [3.9.6]

GHGs differ in their physical characteristics. For example, per unit mass in the atmosphere, methane $\left(\mathrm{CH}_{4}\right)$ causes a stronger instantaneous radiative forcing than $\mathrm{CO}_{2}$, but it remains in the atmosphere for a much shorter time. Thus, the time profiles of climate change brought about by different GHGs are different and consequential. Determining how emissions of different GHGs are compared for mitigation purposes involves comparing the resulting temporal profiles of climate change from each gas and making value judgments about the relative significance to humans of these profiles, which is a process fraught with uncertainty. [3.9.6; WGI 8.7]

A commonly used metric is the Global Warming Potential (GWP). It is defined as the accumulated radiative forcing within a specific time horizon (e. g., 100 years - $\mathrm{GWP}_{100}$ ), caused by emitting one kilogram of the gas, relative to that of the reference gas $\mathrm{CO}_{2}$. This metric is used to transform the effects of different $\mathrm{GHG}$ emissions to a common scale ( $\mathrm{CO}_{2}$-equivalents). ' One strength of the GWP is

In this summary, all quantities of $\mathrm{GHG}$ emissions are expressed in $\mathrm{CO}_{2}$-equivalent $\left(\mathrm{CO}_{2}\right.$ eq) emissions that are calculated based on $\mathrm{GWP}_{100}$. Unless otherwise stated, GWP values for different gases are taken from IPCC Second Assessment Report (SAR). Although GWP values have been updated several times since, the SAR values are widely used in policy settings, including the Kyoto Protocol, as well as in many national and international emission accounting systems. Modelling studies show that the changes in $\mathrm{GWP}_{100}$ values from SAR to AR4 have little impact on the optimal mitigation strategy at the global level. [6.3.2.5, Annex II.9.1] that it can be calculated in a relatively transparent and straightforward manner. However, there are also limitations, including the requirement to use a specific time horizon, the focus on cumulative forcing, and the insensitivity of the metric to the temporal profile of climate effects and its significance to humans. The choice of time horizon is particularly important for short-lived gases, notably methane: when computed with a shorter time horizon for GWP, their share in calculated total warming effect is larger and the mitigation strategy might change as a consequence. [1.2.5]

Many alternative metrics have been proposed in the scientific literature. All of them have advantages and disadvantages, and the choice of metric can make a large difference for the weights given to emissions from particular gases. For instance, methane's $\mathrm{GWP}_{100}$ is 28 while its Global Temperature Change Potential (GTP), one alternative metric, is 4 for the same time horizon (AR5 values, see WGI Section 8.7). In terms of aggregate mitigation costs alone, GWP ${ }_{100}$ may perform similarly to other metrics (such as the time-dependent Global Temperature Change Potential or the Global Cost Potential) of reaching a prescribed climate target; however, there may be significant differences in terms of the implied distribution of costs across sectors, regions, and over time. [3.9.6, 6.3.2.5]

An alternative to a single metric for all gases is to adopt a 'multibasket' approach in which gases are grouped according to their contributions to short and long term climate change. This may solve some problems associated with using a single metric, but the question remains of what relative importance to attach to reducing GHG emissions in the different groups. [3.9.6; WGI 8.7]

\section{TS.2.2 Greenhouse gas emission drivers}

This section examines the factors that have, historically, been associated with changes in GHG emissions levels. Typically, such analysis is based on a decomposition of total GHG emissions into various components such as growth in the economy (Gross Domestic Product (GDP)/capita), growth in the population (capita), the energy intensity needed per unit of economic output (energy/GDP) and the GHG emissions intensity of that energy (GHGs/energy). As a practical matter, due to data limitations and the fact that most $\mathrm{GHG}$ emissions take the form of $\mathrm{CO}_{2}$ from industry and energy, almost all this research focuses on $\mathrm{CO}_{2}$ from those sectors.

Globally, economic and population growth continue to be the most important drivers of increases in $\mathrm{CO}_{2}$ emissions from fossil fuel combustion. The contribution of population growth between 2000 and 2010 remained roughly identical to the previous three decades, while the contribution of economic growth has risen sharply (high confidence). Worldwide population increased by $86 \%$ between 1970 and 2010 , from 3.7 to 6.9 billion. Over the same period, income as measured through production and/ or consumption per capita has grown by a factor of about two. The exact measurement of global economic growth is difficult because countries use different currencies and converting 
individual national economic figures into global totals can be done in various ways. With rising population and economic output, emissions of $\mathrm{CO}_{2}$ from fossil fuel combustion have risen as well. Over the last decade, the importance of economic growth as a driver of global $\mathrm{CO}_{2}$ emissions has risen sharply while population growth has remained roughly steady. Due to changes in technology, changes in the economic structure and the mix of energy sources as well as changes in other inputs such as capital and labour, the energy intensity of economic output has steadily declined worldwide. This decline has had an offsetting effect on global $\mathrm{CO}_{2}$ emissions that is nearly of the same magnitude as growth in population (Figure TS.6). There are only a few countries that combine economic growth and decreasing territorial $\mathrm{CO}_{2}$ emissions over longer periods of time. Such decoupling remains largely atypical, especially when considering consumption-based $\mathrm{CO}_{2}$ emissions. [1.3, 5.3]

Between 2000 and 2010, increased use of coal relative to other energy sources has reversed a long-standing pattern of gradual decarbonization of the world's energy supply (high confidence). Increased use of coal, especially in developing Asia, is exacerbating the burden of energy-related GHG emissions (Figure TS.6). Estimates

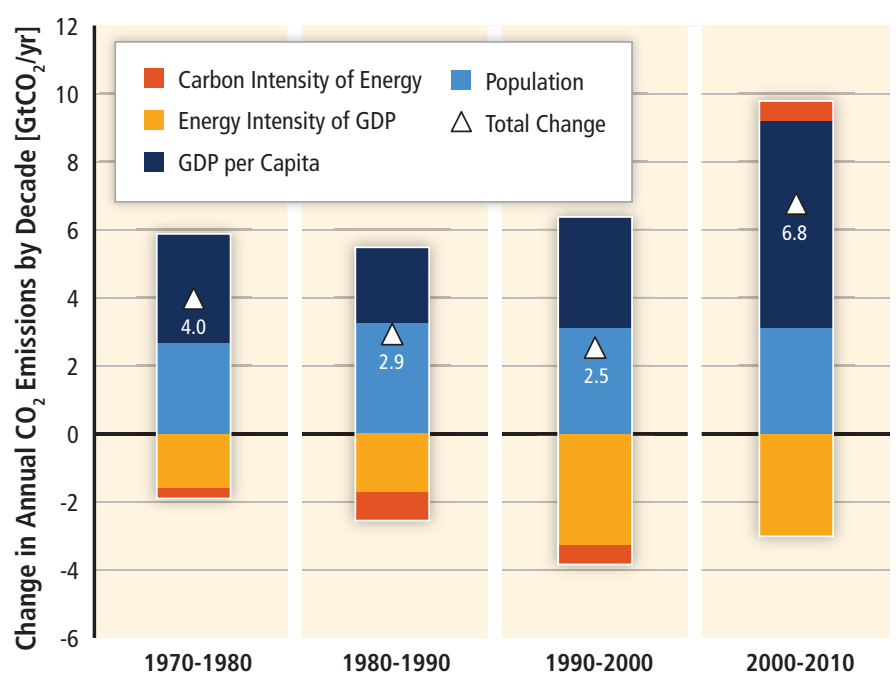

Figure TS.6 Decomposition of the change in total annual $\mathrm{CO}_{2}$ emissions from fossil fuel combustion by decade and four driving factors: population, income (GDP) per capita, energy intensity of GDP and carbon intensity of energy. Total emissions changes are indicated by a triangle. The change in emissions over each decade is measured in gigatonnes of $\mathrm{CO}_{2}$ per year $\left(\mathrm{GtCO}_{2} / \mathrm{yr}\right)$; income is converted into common units using purchasing power parities. [Figure 1.7]

\section{Box TS.6 | The use of scenarios in this report}

Scenarios of how the future might evolve capture key factors of human development that influence GHG emissions and our ability to respond to climate change. Scenarios cover a range of plausible futures, because human development is determined by a myriad of factors including human decision making. Scenarios can be used to integrate knowledge about the drivers of GHG emissions, mitigation options, climate change, and climate impacts.

One important element of scenarios is the projection of the level of human interference with the climate system. To this end, a set of four 'representative concentration pathways' (RCPs) has been developed. These RCPs reach radiative forcing levels of 2.6, 4.5, 6.0 , and 8.5 Watts per square meter $\left(\mathrm{W} / \mathrm{m}^{2}\right)$ (corresponding to concentrations of $450,650,850$, and $1370 \mathrm{ppm} \mathrm{CO} 2 \mathrm{eq}$ ), respectively, in 2100 , covering the range of anthropogenic climate forcing in the 21st century as reported in the literature. The four RCPs are the basis of a new set of climate change projections that have been assessed by WGI AR5. [WGI 6.4, WGI 12.4]

Scenarios of how the future develops without additional and explicit efforts to mitigate climate change ('baseline scenarios') and with the introduction of efforts to limit GHG emissions ('mitigation scenarios'), respectively, generally include socio-economic projections in addition to emission, concentration, and climate change information. WGIII AR5 has assessed the full breadth of baseline and mitigation scenarios in the literature. To this end, it has collected a database of more than 1200 published mitigation and baseline scenarios. In most cases, the underlying socio-economic projections reflect the modelling teams' individual choices about how to conceptualize the future in the absence of climate policy. The baseline scenarios show a wide range of assumptions about economic growth (ranging from threefold to more than eightfold growth in per capita income by 2100 ), demand for energy (ranging from a $40 \%$ to more than $80 \%$ decline in energy intensity by 2100) and other factors, in particular the carbon intensity of energy. Assumptions about population are an exception: the vast majority of scenarios focus on the low to medium population range of nine to 10 billion people by 2100 . Although the range of emissions pathways across baseline scenarios in the literature is broad, it may not represent the full potential range of possibilities (Figure TS.7). [6.3.1]

The concentration outcomes of the baseline and mitigation scenarios assessed by WGIII AR5 cover the full range of RCPs. However, they provide much more detail at the lower end, with many scenarios aiming at concentration levels in the range of 450 , 500 , and $550 \mathrm{ppm} \mathrm{CO} \mathrm{C}_{2}$ eq in 2100 . The climate change projections of WGI based on RCPs, and the mitigation scenarios assessed by WGIII AR5 can be related to each other through the climate outcomes they imply. [6.2.1] 

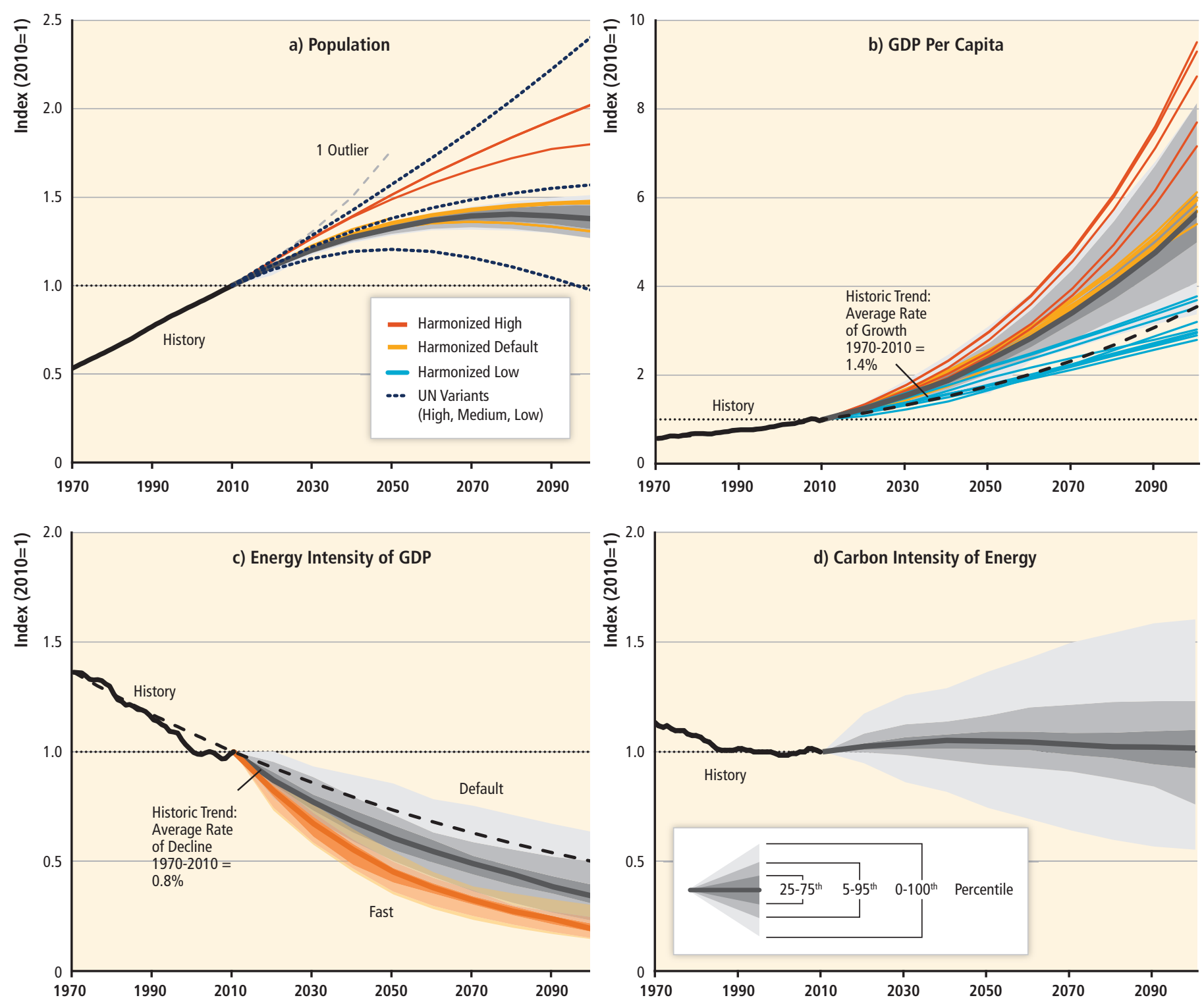

Figure TS.7| Global baseline projection ranges for four emissions driving factors. Scenarios harmonized with respect to a particular factor are depicted with individual lines. Other scenarios are depicted as a range with median emboldened; shading reflects interquartile range (darkest), 5th-95th percentile range (lighter), and full range (lightest), excluding one indicated outlier in panel a). Scenarios are filtered by model and study for each indicator to include only unique projections. Model projections and historic data are normalized to 1 in 2010. GDP is aggregated using base-year market exchange rates. Energy and carbon intensity are measured with respect to total primary energy. [Figure 6.1]

indicate that coal and unconventional gas and oil resources are large; therefore reducing the carbon intensity of energy may not be primarily driven by fossil resource scarcity, but rather by other driving forces such as changes in technology, values, and socio-political choices. [5.3, 7.2, 7.3, 7.4; SRREN Figure 1.7]

Technological innovations, infrastructural choices, and behaviour affect GHG emissions through productivity growth, energyand carbon-intensity and consumption patterns (medium confidence). Technological innovation improves labour and resource productivity; it can support economic growth both with increasing and with decreasing GHG emissions. The direction and speed of technological change depends on policies. Technology is also central to the choices of infrastructure and spatial organization, such as in cities, which can have long-lasting effects on GHG emissions. In addition, a wide array of attitudes, values, and norms can inform different lifestyles, consumption preferences, and technological choices all of which, in turn, affect patterns of GHG emissions. [5.3, 5.5, 5.6, 12.3]

Without additional efforts to reduce GHG emissions beyond those in place today, emissions growth is expected to persist, driven by growth in global population and economic activities despite improvements in energy supply and end-use technologies (high confidence). Atmospheric concentrations in baseline scenarios collected for this assessment (scenarios without explicit additional efforts to reduce GHG emissions) exceed 450 parts per million 
(ppm) $\mathrm{CO}_{2}$ eq by $2030 .^{7}$ They reach $\mathrm{CO}_{2}$ eq concentration levels from 750 to more than $1300 \mathrm{ppm} \mathrm{CO}_{2} \mathrm{eq}$ by 2100 and result in projected global mean surface temperature increases in 2100 from 3.7 to $4.8^{\circ} \mathrm{C}$ compared to pre-industrial levels ${ }^{8}$ (range based on median climate response; the range is $2.5^{\circ} \mathrm{C}$ to $7.8^{\circ} \mathrm{C}$ when including climate uncertainty, see Table TS.1). ${ }^{9}$ The range of 2100 concentrations corresponds roughly to the range of $\mathrm{CO}_{2}$ eq concentrations in the Representative Concentration Pathways (RCP) 6.0 and RCP8.5 pathways (see Box TS.6), with the majority of scenarios falling below the latter. For comparison, the $\mathrm{CO}_{2} \mathrm{eq}$ concentration in 2011 has been estimated to be $430 \mathrm{ppm}$ (uncertainty range $340-520 \mathrm{ppm}$ ). ${ }^{10}$ The literature does not systematically explore the full range of uncertainty surrounding development pathways and possible evolution of key drivers such as population, technology, and resources. Nonetheless, the scenarios strongly suggest that absent any explicit mitigation efforts, cumulative $\mathrm{CO}_{2}$ emissions since 2010 will exceed $700 \mathrm{GtCO}_{2}$ by $2030,1,500 \mathrm{GtCO}_{2}$ by 2050 , and potentially well over $4,000 \mathrm{GtCO}_{2}$ by 2100 . [6.3.1; WGI Figure SPM.5, WGI 8.5, WGI 12.3]

\section{TS.3 Mitigation pathways and measures in the context of sustainable development}

This section assesses the literature on mitigation pathways and measures in the context of sustainable development. Section TS 3.1 first examines the anthropogenic GHG emissions trajectories and potential temperature implications of mitigation pathways leading to a range of future atmospheric $\mathrm{CO}_{2}$ eq concentrations. It then explores the technological, economic, and institutional requirements of these pathways along with their potential co-benefits and adverse side-effects. Section TS 3.2 examines mitigation options by sector and how they may interact across sectors.

These $\mathrm{CO}_{2}$ eq concentrations represent full radiative forcing, including GHGs, halogenated gases, tropospheric ozone, aerosols, mineral dust and albedo change. 8 Based on the longest global surface temperature dataset available, the observed change between the average of the period 1850-1900 and of the AR5 reference period $(1986-2005)$ is $0.61^{\circ} \mathrm{C}\left(5-95 \%\right.$ confidence interval: 0.55 to $\left.0.67^{\circ} \mathrm{C}\right)$ [WGI SPM.E], which is used here as an approximation of the change in global mean surface temperature since pre-industrial times, referred to as the period before 1750 .

9 Provided estimates reflect the 10th to the 90th percentile of baseline scenarios collected for this assessment. The climate uncertainty reflects the 5th to 95th percentile of climate model calculations described in Table TS.1 for each scenario.

10 This is based on the assessment of total anthropogenic radiative forcing for 2011 relative to 1750 in WGI AR5, i.e., $2.3 \mathrm{~W} \mathrm{~m}^{-2}$, uncertainty range 1.1 to $3.3 \mathrm{~W} \mathrm{~m}^{-2}$. [WGI Figure SPM.5, WGI 8.5, WGI 12.3]

\section{TS.3.1 Mitigation pathways}

\section{TS.3.1.1 Understanding mitigation pathways in the context of multiple objectives}

The world's societies will need to both mitigate and adapt to climate change if it is to effectively avoid harmful climate impacts (robust evidence, high agreement). There are demonstrated examples of synergies between mitigation and adaptation [11.5.4, 12.8.1] in which the two strategies are complementary. More generally, the two strategies are related because increasing levels of mitigation imply less future need for adaptation. Although major efforts are now underway to incorporate impacts and adaptation into mitigation scenarios, inherent difficulties associated with quantifying their interdependencies have limited their representation in models used to generate mitigation scenarios assessed in WGIII AR5 (Box TS.7). [2.6.3, 3.7.2.1, 6.3.3]

There is no single pathway to stabilize $\mathrm{CO}_{2} \mathrm{eq}$ concentrations at any level; instead, the literature points to a wide range of mitigation pathways that might meet any concentration level (high confidence). Choices, whether deliberated or not, will determine which of these pathways is followed. These choices include, among other things, the emissions pathway to bring atmospheric $\mathrm{CO}_{2}$ eq concentrations to a particular level, the degree to which concentrations temporarily exceed (overshoot) the long-term level, the technologies that are deployed to reduce emissions, the degree to which mitigation is coordinated across countries, the policy approaches used to achieve mitigation within and across countries, the treatment of land use, and the manner in which mitigation is meshed with other policy objectives such as sustainable development. A society's development pathway-with its particular socioeconomic, institutional, political, cultural and technological features-enables and constrains the prospects for mitigation. At the national level, change is considered most effective when it reflects country and local visions and approaches to achieving sustainable development according to national circumstances and priorities. [4.2, 6.3-6.8, 11.8]

Mitigation pathways can be distinguished from one another by a range of outcomes or requirements (high confidence). Decisions about mitigation pathways can be made by weighing the requirements of different pathways against each other. Although measures of aggregate economic costs and benefits have often been put forward as key decision-making factors, they are far from the only outcomes that matter. Mitigation pathways inherently involve a range of synergies and tradeoffs connected with other policy objectives such as energy and food security, energy access, the distribution of economic impacts, local air quality, other environmental factors associated with different technological solutions, and economic competitiveness (Box TS.11). Many of these fall under the umbrella of sustainable development. In addition, requirements such as the rates of up-scaling of energy technologies or the rates of reductions in GHG emissions may provide important insights into the degree of challenge associated with meeting a particular long-term goal. [4.5, 4.8, 6.3, 6.4, 6.6] 


\section{Box TS.7 | Scenarios from integrated models can help to understand how actions affect outcomes in complex systems}

The long-term scenarios assessed in this report were generated primarily by large-scale computer models, referred to here as 'integrated models', because they attempt to represent many of the most important interactions among technologies, relevant human systems (e.g., energy, agriculture, the economic system), and associated GHG emissions in a single integrated framework. A subset of these models is referred to as 'integrated assessment models', or IAMs. IAMs include not only an integrated representation of human systems, but also of important physical processes associated with climate change, such as the carbon cycle, and sometimes representations of impacts from climate change. Some IAMs have the capability of endogenously balancing impacts with mitigation costs, though these models tend to be highly aggregated. Although aggregate models with representations of mitigation and damage costs can be very useful, the focus in this assessment is on integrated models with sufficient sectoral and geographic resolution to understand the evolution of key processes such as energy systems or land systems.

Scenarios from integrated models are invaluable to help understand how possible actions or choices might lead to different future outcomes in these complex systems. They provide quantitative, long-term projections (conditional on our current state of knowledge) of many of the most important characteristics of mitigation pathways while accounting for many of the most important interactions between the various relevant human and natural systems. For example, they provide both regional and global information about emissions pathways, energy and landuse transitions, and aggregate economic costs of mitigation.

At the same time, these integrated models have particular characteristics and limitations that should be considered when interpreting their results. Many integrated models are based on the rational choice paradigm for decision making, excluding the consideration of some behavioural factors. The models approximate cost-effective solutions that minimize the aggregate economic costs of achieving mitigation outcomes, unless they are specifically constrained to behave otherwise. Scenarios from these models capture only some of the dimensions of development pathways that are relevant to mitigation options, often only minimally treating issues such as distributional impacts of mitigation actions and consistency with broader development goals. In addition, the models in this assessment do not effectively account for the interactions between mitigation, adaptation, and climate impacts. For these reasons, mitigation has been assessed independently from climate impacts. Finally, and most fundamentally, integrated models are simplified, stylized, numerical approaches for representing enormously complex physical and social systems, and scenarios from these models are based on uncertain projections about key events and drivers over often century-long timescales. Simplifications and differences in assumptions are the reason why output generated from different models-or versions of the same model-can differ, and projections from all models can differ considerably from the reality that unfolds. $[3.7,6.2]$
TS.3.1.2 Short- and long-term requirements of mitigation pathways

Mitigation scenarios point to a range of technological and behavioral measures that could allow the world's societies to follow GHG emissions pathways consistent with a range of different levels of mitigation (high confidence). As part of this assessment, about 900 mitigation and 300 baseline scenarios have been collected from integrated modelling research groups around the world (Box TS.7). The mitigation scenarios span atmospheric concentration levels in 2100 from $430 \mathrm{ppm} \mathrm{CO}_{2}$ eq to above $720 \mathrm{ppm} \mathrm{CO}_{2} \mathrm{eq}$, which is roughly comparable to the 2100 forcing levels between the RCP2.6 and RCP6.0 scenarios (Figure TS.8, left panel). Scenarios have been constructed to reach mitigation goals under very different assumptions about energy demands, international cooperation, technologies, the contributions of $\mathrm{CO}_{2}$ and other forcing agents to atmospheric $\mathrm{CO}_{2}$ eq concentrations, and the degree to which concentrations temporarily exceed the long-term goal (concentration overshoot, see Box TS.8). Other scenarios were also assessed, including some scenarios with concentrations in 2100 below $430 \mathrm{ppm} \mathrm{CO}$ eq (for a discussion of these scenarios see below). [6.3]

Limiting atmospheric peak concentrations over the course of the century-not only reaching long-term concentration levels-is critical for limiting transient temperature change (high confidence). Scenarios reaching concentration levels of about $500 \mathrm{ppm}$ $\mathrm{CO}_{2}$ eq by 2100 are more likely than not to limit temperature change to less than $2{ }^{\circ} \mathrm{C}$ relative to pre-industrial levels, unless they temporarily 'overshoot' concentration levels of roughly $530 \mathrm{ppm} \mathrm{CO}_{2}$ eq before 2100. In this case, they are about as likely as not to achieve that goal. The majority of scenarios reaching long-term concentrations of about $450 \mathrm{ppm} \mathrm{CO}_{2}$ eq in 2100 are likely to keep temperature change below $2{ }^{\circ} \mathrm{C}$ over the course of the century relative to pre-industrial levels (Table TS.1, Box TS.8). Scenarios that reach 530 to $650 \mathrm{ppm} \mathrm{CO}$ eq concentrations by 2100 are more unlikely than likely to keep temperature change below $2{ }^{\circ} \mathrm{C}$ relative to pre-industrial levels. Scenarios that exceed about $650 \mathrm{ppm} \mathrm{CO} \mathrm{CO}_{2} \mathrm{eq}$ by 2100 are unlikely to limit temperature change to below $2{ }^{\circ} \mathrm{C}$ relative to pre-industrial levels. Mitigation 
Total GHG Emissions in all AR5 Scenarios

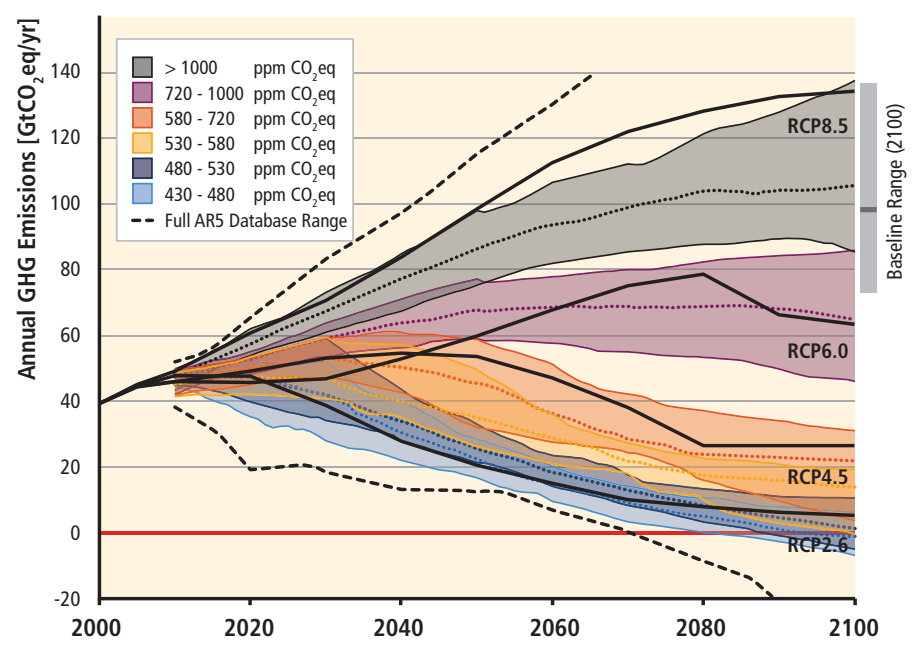

GHG Emissions with Different Assumptions for Negative Emissions

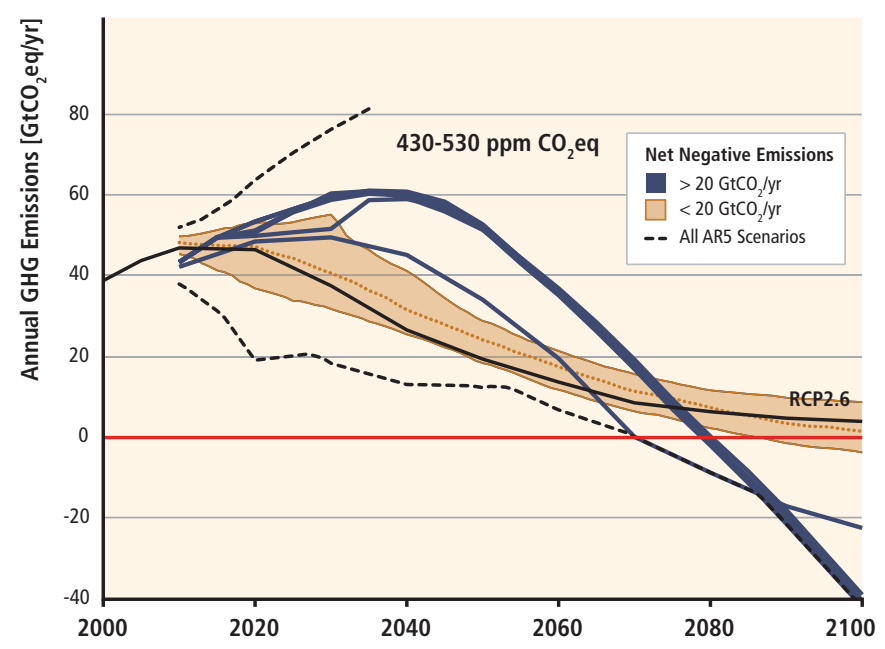

Figure TS.8| Development of total GHG emissions for different long-term concentration levels (left panel) and for scenarios reaching about 450 to about 500 (430-530) ppm $\mathrm{CO}_{2}$ eq in 2100 with and without net negative $\mathrm{CO}_{2}$ emissions larger than $20 \mathrm{GtCO}_{2} / \mathrm{yr}$ (right panel). Ranges are given for the 10th-90th percentile of scenarios. [Figure 6.7]

scenarios in which temperature increase is more likely than not to be less than $1.5^{\circ} \mathrm{C}$ relative to pre-industrial levels by 2100 are characterized by concentrations in 2100 of below 430 ppm $\mathrm{CO}_{2}$ eq. Temperature peaks during the century and then declines in these scenarios. [6.3]

Mitigation scenarios reaching about 450 ppm $\mathrm{CO}_{2} \mathrm{eq}$ in 2100 typically involve temporary overshoot of atmospheric concentrations, as do many scenarios reaching about $500 \mathrm{ppm}$ or about 550 ppm $\mathrm{CO}_{2}$ eq in 2100 (high confidence). Concentration overshoot means that concentrations peak during the century before descending toward their 2100 levels. Overshoot involves less mitigation in the near term, but it also involves more rapid and deeper emissions reductions in the long run. The vast majority of scenarios reaching about $450 \mathrm{ppm} \mathrm{CO}$ eq in 2100 involve concentration overshoot, since most models cannot reach the immediate, near-term emissions reductions that would be necessary to avoid overshoot of these concentration levels. Many scenarios have been constructed to reach about $550 \mathrm{ppm}$ $\mathrm{CO}_{2}$ eq by 2100 without overshoot.

Depending on the level of overshoot, many overshoot scenarios rely on the availability and widespread deployment of bioenergy with carbon dioxide capture and storage (BECCS) and/or afforestation in the second half of the century (high confidence). These and other carbon dioxide removal (CDR) technologies and methods remove $\mathrm{CO}_{2}$ from the atmosphere (negative emissions). Scenarios with overshoot of greater than $0.4 \mathrm{~W} / \mathrm{m}^{2}$ (> 35-50 ppm $\mathrm{CO}_{2}$ eq concentration) typically deploy $\mathrm{CDR}$ technologies to an extent that net global $\mathrm{CO}_{2}$ emissions become negative in the second-half of the century (Figure TS.8, right panel). CDR is also prevalent in many scenarios without concentration overshoot to compensate for residual emissions from sectors where mitigation is more expensive. The availability and potential of BECCS, afforestation, and other CDR technolo- gies and methods are uncertain and CDR technologies and methods are, to varying degrees, associated with challenges and risks. There is uncertainty about the potential for large-scale deployment of BECCS, large-scale afforestation, and other CDR technologies and methods. $[6.3,6.9]$

Reaching atmospheric concentration levels of about 450 to about 500 ppm $\mathrm{CO}_{2}$ eq by 2100 will require substantial cuts in anthropogenic GHG emissions by mid-century (high confidence). Scenarios reaching about $450 \mathrm{ppm} \mathrm{CO}_{2}$ eq by 2100 are associated with GHG emissions reductions of about $40 \%$ to $70 \%$ by 2050 compared to 2010 and emissions levels near zero $\mathrm{GtCO}_{2}$ eq or below in $2100 .{ }^{11}$ Scenarios with GHG emissions reductions in 2050 at the lower end of this range are characterized by a greater reliance on CDR technologies beyond midcentury. The majority of scenarios that reach about $500 \mathrm{ppm} \mathrm{CO}_{2} \mathrm{eq}$ in 2100 without overshooting roughly 530 ppm $\mathrm{CO}_{2} \mathrm{eq}$ at any point during the century are associated with GHG emissions reductions of $40 \%$ to $55 \%$ by 2050 compared to 2010 (Figure TS.8, left panel; Table TS.1). In contrast, in some scenarios in which concentrations rise to well above $530 \mathrm{ppm} \mathrm{CO}$ eq during the century before descending to concentrations below this level by 2100, emissions rise to as high as 20\% above 2010 levels in 2050. However, these high-overshoot scenarios are characterized by negative global emissions of well over $20 \mathrm{GtCO}_{2}$ per year in the second half of the century (Figure TS.8, right panel). Cumulative $\mathrm{CO}_{2}$

This range differs from the range provided for a similar concentration category in AR4 ( $50 \%$ to $85 \%$ lower than 2000 for $\mathrm{CO}_{2}$ only). Reasons for this difference include that this report has assessed a substantially larger number of scenarios than in AR4 and looks at all GHGs. In addition, a large proportion of the new scenarios include Carbon Dioxide Removal (CDR) technologies and associated increases in concentration overshoot. Other factors include the use of 2100 concentration levels instead of stabilization levels and the shift in reference year from 2000 to 2010. 


\section{Box TS.8 | Assessment of temperature change in the context of mitigation scenarios}

Long-term climate goals have been expressed both in terms of concentrations and temperature. Article 2 of the UNFCCC calls for the need to 'stabilize' concentrations of GHGs. Stabilization of concentrations is generally understood to mean that the $\mathrm{CO}_{2}$ eq concentration reaches a specific level and then remains at that level indefinitely until the global carbon and other cycles come into a new equilibrium. The notion of stabilization does not necessarily preclude the possibility that concentrations might exceed, or 'overshoot' the long-term goal before eventually stabilizing at that goal. The possibility of 'overshoot' has important implications for the required GHG emissions reductions to reach a long-term concentration level. Concentration overshoot involves less mitigation in the near term with more rapid and deeper emissions reductions in the long run.

The temperature response of the concentration pathways assessed in this report focuses on transient temperature change over the course of the century. This is an important difference with WGIII AR4, which focused on the long-term equilibrium temperature response, a state that is reached millennia after the stabilization of concentrations. The temperature outcomes in this report are thus not directly comparable to those presented in the WGIII AR4 assessment. One reason that this assessment focuses on transient temperature response is that it is less uncertain than the equilibrium response and correlates more strongly with GHG emissions in the near and medium term. An additional reason is that the mitigation pathways assessed in WGIII AR5 do not extend beyond 2100 and are primarily designed to reach specific concentration goals for the year 2100. The majority of these pathways do not stabilize concentrations in 2100 , which makes the assessment of the equilibrium temperature response ambiguous and dependent on assumptions about post-2100 emissions and concentrations.
Transient temperature goals might be defined in terms of the temperature in a specific year (e. g., 2100), or based on never exceeding a particular level. This report explores the implications of both types of goals. The assessment of temperature goals are complicated by the uncertainty that surrounds our understanding of key physical relationships in the earth system, most notably the relationship between concentrations and temperature. It is not possible to state definitively whether any long-term concentration pathway will limit either transient or equilibrium temperature change to below a specified level. It is only possible to express the temperature implications of particular concentration pathways in probabilistic terms, and such estimates will be dependent on the source of the probability distribution of different climate parameters and the climate model used for analysis. This report employs the MAGICC model and a distribution of climate parameters that results in temperature outcomes with dynamics similar to those from the Earth System Models assessed in WGI AR5. For each emissions scenario, a median transient temperature response is calculated to illustrate the variation of temperature due to different emissions pathways. In addition, a transient temperature range for each scenario is provided, reflecting the climate system uncertainties. Information regarding the full distribution of climate parameters was utilized for estimating the likelihood that the scenarios would limit transient temperature change to below specific levels (Table TS.1). Providing the combination of information about the plausible range of temperature outcomes as well as the likelihood of meeting different targets is of critical importance for policymaking, since it facilitates the assessment of different climate objectives from a risk management perspective. [2.5.7.2, 6.3.2] emissions between 2011 and 2100 are $630-1180 \mathrm{GtCO}_{2}$ in scenarios reaching about $450 \mathrm{ppm} \mathrm{CO}_{2}$ eq in 2100 ; they are $960-1550 \mathrm{GtCO}_{2}$ in scenarios reaching about $500 \mathrm{ppm} \mathrm{CO}$ eq in 2100. The variation in cumulative $\mathrm{CO}_{2}$ emissions across scenarios is due to differences in the contribution of non- $\mathrm{CO}_{2} \mathrm{GHGs}$ and other radiatively active substances as well as the timing of mitigation (Table TS.1). [6.3]

In order to reach atmospheric concentration levels of about 450 to about $500 \mathrm{ppm} \mathrm{CO}_{2}$ eq by 2100 , the majority of mitigation relative to baseline emissions over the course of century will occur in the non-Organisation for Economic Co-operation and Development (OECD) countries (high confidence). In scenarios that attempt to cost-effectively allocate emissions reductions across countries and over time, the total $\mathrm{CO}_{2}$ eq emissions reductions from baseline emissions in non-OECD countries are greater than in OECD countries. This is, in large part, because baseline emissions from the non-OECD countries are projected to be larger than those from the OECD countries, but it also derives from higher carbon intensities in non-OECD countries and different terms of trade structures. In these scenarios, GHG emissions peak earlier in the OECD countries than in the nonOECD countries. [6.3]

Reaching atmospheric concentration levels of about 450 to about $650 \mathrm{ppm} \mathrm{CO} \mathrm{CO}_{2}$ eq by 2100 will require large-scale changes to global and national energy systems over the coming decades (high confidence). Scenarios reaching atmospheric concentrations levels of about 450 to about $500 \mathrm{ppm} \mathrm{CO}$ eq by 2100 are characterized by a tripling to nearly a quadrupling of the global share of zero- and lowcarbon energy supply from renewables, nuclear energy, fossil energy with carbon dioxide capture and storage (CCS), and bioenergy with CCS (BECCS), by the year 2050 relative to 2010 (about 17\%) (Figure TS.10, left panel). The increase in total global low-carbon energy sup- 
Table TS.1 | Key characteristics of the scenarios collected and assessed for WGIII AR5. For all parameters, the 10th to 90th percentile of the scenarios is shown. ${ }^{1,2}$ [Table 6.3]

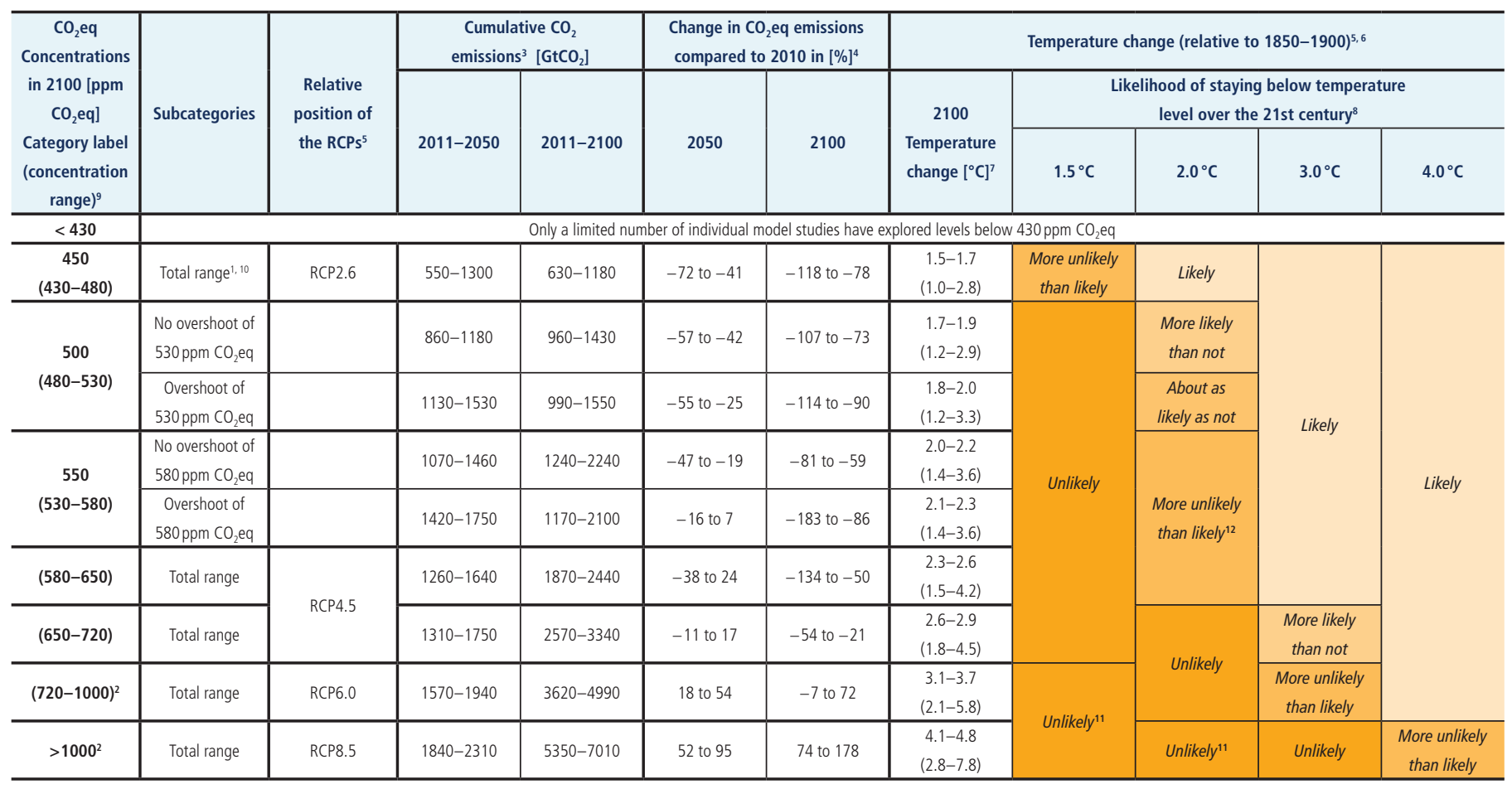

Notes:

1 The 'total range' for the $430-480 \mathrm{ppm} \mathrm{CO}$ eq scenarios corresponds to the range of the 10th-90th percentile of the subcategory of these scenarios shown in Table 6.3 .

2 Baseline scenarios (see TS.2.2) fall into the $>1000$ and $720-1000$ ppm CO $\mathrm{CO}_{2}$ eq categories. The latter category also includes mitigation scenarios. The baseline scenarios in the latter category reach a temperature change of $2.5-5.8^{\circ} \mathrm{C}$ above preindustrial in 2100 . Together with the baseline scenarios in the $>1000 \mathrm{ppm} \mathrm{CO}_{2} \mathrm{eq} \mathrm{category}$, this leads to an overall 2100 temperature range of $2.5-7.8^{\circ} \mathrm{C}$ (range based on median climate response: $3.7-4.8^{\circ} \mathrm{C}$ ) for baseline scenarios across both concentration categories.

3 For comparison of the cumulative $\mathrm{CO}_{2}$ emissions estimates assessed here with those presented in WGI AR5, an amount of 515 [445-585] GtC (1890 [1630-2150] GtCO $)$, was already emitted by 2011 since 1870 [WGI 12.5]. Note that cumulative $\mathrm{CO}_{2}$ emissions are presented here for different periods of time (2011-2050 and 2011-2100) while cumulative $\mathrm{CO}_{2}$ emissions in WGI AR5 are presented as total compatible emissions for the RCPs (2012-2100) or for total compatible emissions for remaining below a given temperature target with a given likelihood [WGI Table SPM.3, WGI SPM.E.8].

4 The global 2010 emissions are $31 \%$ above the 1990 emissions (consistent with the historic $\mathrm{GHG}$ emissions estimates presented in this report). $\mathrm{CO}_{2}$ eq emissions include the basket of Kyoto gases $\left(\mathrm{CO}_{2}, \mathrm{CH}_{4}, \mathrm{~N}_{2} \mathrm{O}\right.$ as well as $\mathrm{F}$-gases).

5 The assessment in WGIII AR5 involves a large number of scenarios published in the scientific literature and is thus not limited to the RCPs. To evaluate the $\mathrm{CO}_{2}$ eq concentration and climate implications of these scenarios, the MAGICC model was used in a probabilistic mode (see Annex II). For a comparison between MAGICC model results and the outcomes of the models used in WGI, see Sections WGI 12.4.1.2, WGI 12.4.8 and 6.3.2.6. Reasons for differences with WGI SPM Table.2 include the difference in reference year (1986-2005 vs. 1850-1900 here), difference in reporting year (2081-2100 vs 2100 here), set-up of simulation (CMIP5 concentration-driven versus MAGICC emission-driven here), and the wider set of scenarios (RCPs versus the full set of scenarios in the WGIII AR5 scenario database here).

6 Temperature change is reported for the year 2100, which is not directly comparable to the equilibrium warming reported in WGIII AR4 [Table 3.5, Chapter 3; see also WGIII AR5 6.3.2]. For the 2100 temperature estimates, the transient climate response (TCR) is the most relevant system property. The assumed $90 \%$ range of the TCR for MAGICC is $1.2-2.6^{\circ} \mathrm{C}$ (median $1.8^{\circ} \mathrm{C}$ ). This compares to the $90 \%$ range of TCR between $1.2-2.4^{\circ} \mathrm{C}$ for CMIP5 [WGI 9.7] and an assessed likely range of $1-2.5^{\circ} \mathrm{C}$ from multiple lines of evidence reported in the WGI AR5 [Box 12.2 in Section 12.5].

7 Temperature change in 2100 is provided for a median estimate of the MAGICC calculations, which illustrates differences between the emissions pathways of the scenarios in each category. The range of temperature change in the parentheses includes in addition the carbon cycle and climate system uncertainties as represented by the MAGICC model [see 6.3.2.6 for further details]. The temperature data compared to the 1850-1900 reference year was calculated by taking all projected warming relative to 1986-2005, and adding $0.61^{\circ} \mathrm{C}$ for 1986-2005 compared to 1850-1900, based on HadCRUT4 [see WGI Table SPM.2].

8 The assessment in this table is based on the probabilities calculated for the full ensemble of scenarios in WGIII AR5 using MAGICC and the assessment in WGI AR5 of the uncertainty of the temperature projections not covered by climate models. The statements are therefore consistent with the statements in WGI AR5, which are based on the CMIP5 runs of the RCPs and the assessed uncertainties. Hence, the likelihood statements reflect different lines of evidence from both WGs. This WGI method was also applied for scenarios with intermediate concentration levels where no CMIP5 runs are available. The likelihood statements are indicative only [6.3], and follow broadly the terms used by the WGI AR5 SPM for temperature projections: likely $66-100 \%$, more likely than not $>50-100 \%$, about as likely as not 33-66 \%, and unlikely 0-33\%. In addition the term more unlikely than likely $0-<50 \%$ is used.

9 The $\mathrm{CO}_{2}$-equivalent concentration includes the forcing of all GHGs including halogenated gases and tropospheric ozone, as well as aerosols and albedo change (calculated on the basis of the total forcing from a simple carbon cycle/climate model, MAGICC).

10 The vast majority of scenarios in this category overshoot the category boundary of $480 \mathrm{ppm} \mathrm{CO}_{2}$ eq concentrations.

11 For scenarios in this category no CMIP5 run [WGI Chapter 12, Table 12.3] as well as no MAGICC realization [6.3] stays below the respective temperature level. Still, an unlikely assignment is given to reflect uncertainties that might not be reflected by the current climate models.

12 Scenarios in the 580-650 ppm $\mathrm{CO}_{2}$ eq category include both overshoot scenarios and scenarios that do not exceed the concentration level at the high end of the category (like RCP4.5). The latter type of scenarios, in general, have an assessed probability of more unlikely than likely to stay below the $2^{\circ} \mathrm{C}$ temperature level, while the former are mostly assessed to have an unlikely probability of staying below this level. 
ply is from three-fold to seven-fold over this same period. Many models could not reach 2100 concentration levels of about $450 \mathrm{ppm} \mathrm{CO}_{2} \mathrm{eq}$ if the full suite of low-carbon technologies is not available. Studies indicate a large potential for energy demand reductions, but also indicate that demand reductions on their own would not be sufficient to bring about the reductions needed to reach levels of about $650 \mathrm{ppm}$ $\mathrm{CO}_{2}$ eq or below by 2100 . $[6.3,7.11]$

Mitigation scenarios indicate a potentially critical role for landrelated mitigation measures and that a wide range of alternative land transformations may be consistent with similar concentration levels (medium confidence). Land-use dynamics in mitigation scenarios are heavily influenced by the production of bioenergy and the degree to which afforestation is deployed as a negativeemissions, or CDR option. They are, in addition, influenced by forces independent of mitigation such as agricultural productivity improvements and increased demand for food. The range of land-use transformations depicted in mitigation scenarios reflects a wide range of differing assumptions about the evolution of all of these forces. Many scenarios reflect strong increases in the degree of competition for land between food, feed, and energy uses. [6.3, 6.8, 11.4.2]

Delaying mitigation efforts beyond those in place today through 2030 will increase the challenges of, and reduce the options for, limiting atmospheric concentration levels from about 450 to about $500 \mathrm{ppm} \mathrm{CO}_{2} \mathrm{eq}$ by the end of the century (high confidence). Cost-effective mitigation scenarios leading to atmospheric concentration levels of about 450 to about 500 ppm $\mathrm{CO}_{2} \mathrm{eq}$ at the end of the 21 st century are typically characterized by annual GHG emissions in 2030 of roughly between $30 \mathrm{GtCO}_{2}$ eq and $50 \mathrm{GtCO}_{2}$ eq. Scenarios with emissions above $55 \mathrm{GtCO}_{2}$ eq in 2030 are characterized by substantially higher rates of emissions reductions from 2030 to 2050 (median emissions reductions of about $6 \% / y r$ as compared to just over $3 \% / y r$ ) (Figure TS.9, right panel); much more rapid scale-up of low-carbon energy over this period (more than a tripling compared to a doubling of the low-carbon energy share) (Figure TS.10, right panel);

\section{GHG Emissions Pathways to 2030 of Mitigation Scenarios Reaching 430-530 ppm $\mathrm{CO}_{2}$ eq in 2100}

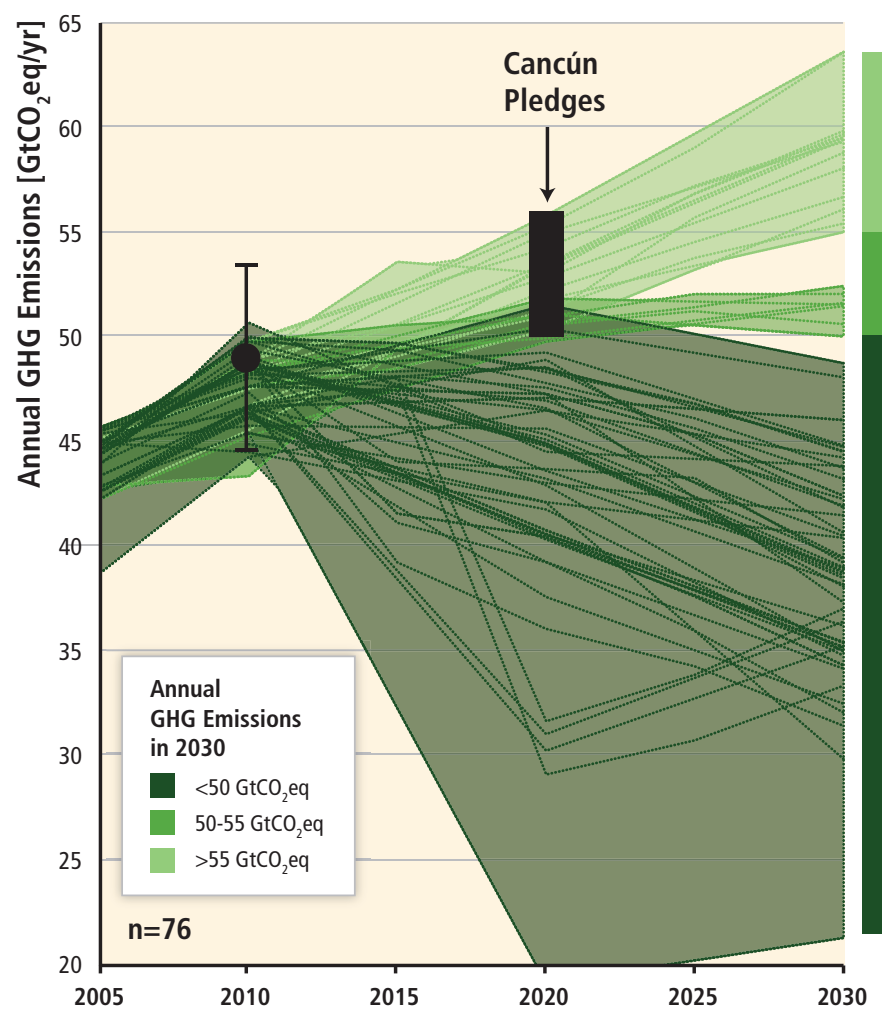

\section{Implications for the Pace of Annual Average $\mathrm{CO}_{2}$ Emissions Reductions from 2030 to 2050 Depending on Different 2030 GHG Emissions Levels}

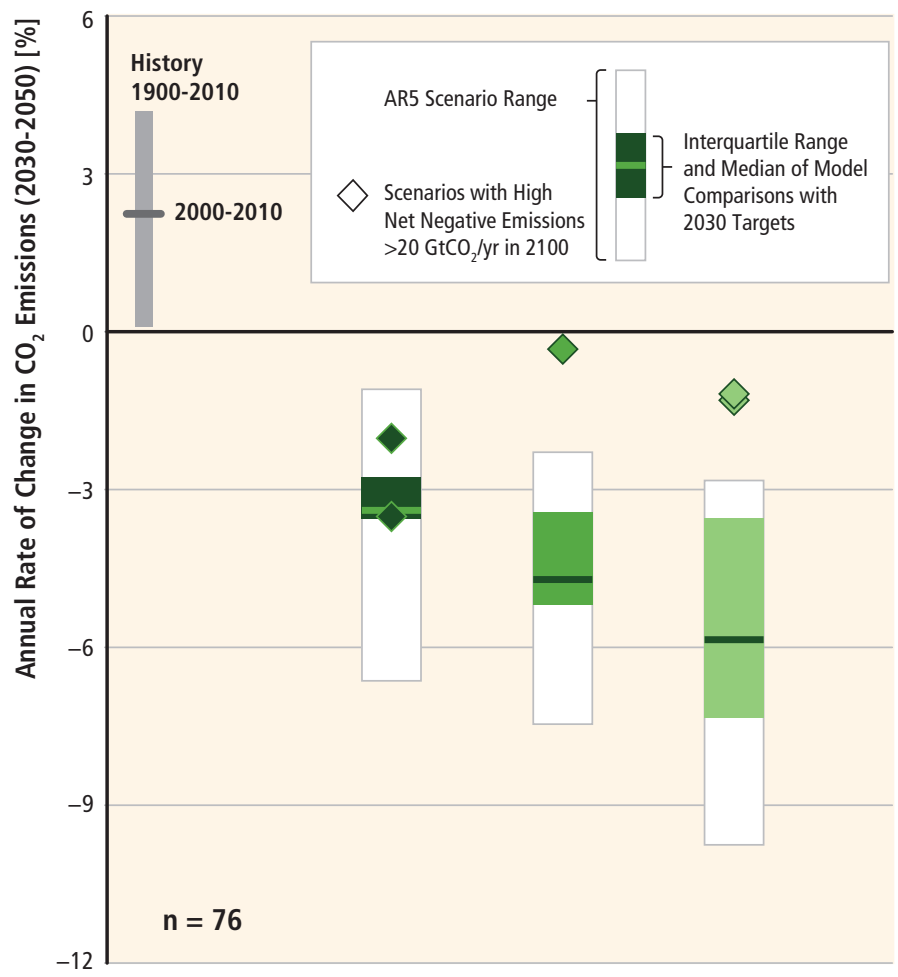

Figure TS.9| The implications of different $2030 \mathrm{GHG}$ emissions levels for the rate of $\mathrm{CO}_{2}$ emissions reductions from 2030 to 2050 in mitigation scenarios reaching about 450 to about 500 (430-530) ppm CO 2 eq concentrations by 2100. The scenarios are grouped according to different emissions levels by 2030 (coloured in different shades of green). The left panel shows the pathways of $\mathrm{GHG}$ emissions ( $\mathrm{GtCO}_{2} \mathrm{eq} / \mathrm{yr}$ ) leading to these 2030 levels. The black bar shows the estimated uncertainty range of $\mathrm{GHG}$ emissions implied by the Cancún Pledges. Black dot with whiskers gives historic GHG emission levels and associated uncertainties in 2010 as reported in Figure TS.1. The right panel denotes the average annual $\mathrm{CO}_{2}$ emissions reduction rates for the period 2030-2050. It compares the median and interquartile range across scenarios from recent intermodel comparisons with explicit 2030 interim goals to the range of scenarios in the Scenario Database for WGIII AR5. Annual rates of historical emissions change between 1900-2010 (sustained over a period of 20 years) and the average annual emissions change between 2000-2010 are shown in grey. Note: Scenarios with large net negative global emissions (> $20 \mathrm{GtCO}_{2} / \mathrm{yr}$ ) are not included in the WGIII AR5 scenario range, but rather shown as independent points. Only scenarios that apply the full, unconstrained mitigation technology portfolio of the underlying models (default technology assumption) are shown. Scenarios with exogenous carbon price assumptions or other policies affecting the timing of mitigation (other than 2030 interim targets) as well as scenarios with 2010 emissions significantly outside the historical range are excluded. [Figure 6.32, 13.13.1.3] 

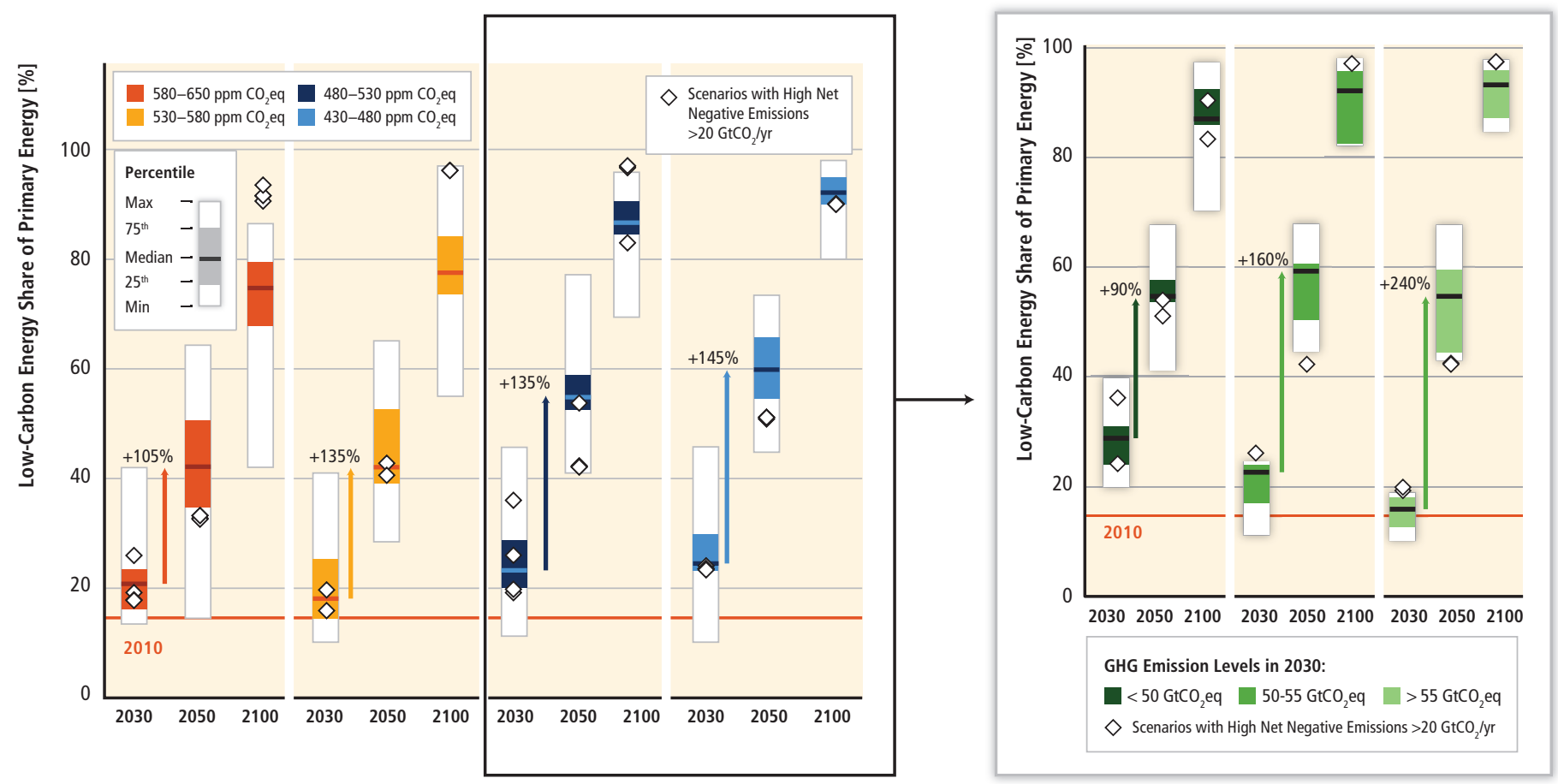

Figure TS.10| The up-scaling of low-carbon energy in scenarios meeting different $2100 \mathrm{CO}_{2}$ eq concentration levels (left panel). The right panel shows the rate of up-scaling subject to different 2030 GHG emissions levels in mitigation scenarios reaching about 450 to about 500 (430-530) ppm $\mathrm{CO}_{2}$ eq concentrations by 2100 . Colored bars show the interquartile range and white bars indicate the full range across the scenarios, excluding those with large, global net negative $\mathrm{CO}_{2}$ emissions (>20 GtCO $/ \mathrm{yr}$ ). Scenarios with large net negative global emissions are shown as individual points. The arrows indicate the magnitude of zero- and low-carbon energy supply up-scaling from 2030 to 2050. Zero- and lowcarbon energy supply includes renewables, nuclear energy, fossil energy with carbon dioxide capture and storage (CCS), and bioenergy with CCS (BECCS). Note: Only scenarios that apply the full, unconstrained mitigation technology portfolio of the underlying models (default technology assumption) are shown. Scenarios with exogenous carbon price assumptions are excluded in both panels. In the right panel, scenarios with policies affecting the timing of mitigation other than 2030 interim targets are also excluded. [Figure 7.16]

a larger reliance on CDR technologies in the long-term (Figure TS.8, right panel); and higher transitional and long term economic impacts (Table TS.2, orange segments, Figure TS.13, right panel). Due to these increased challenges, many models with 2030 GHG emissions in this range could not produce scenarios reaching atmospheric concentrations levels of about 450 to about 500 ppm $\mathrm{CO}_{2}$ eq in 2100. [6.4, 7.11]

Estimated global GHG emissions levels in 2020 based on the Cancún Pledges are not consistent with cost-effective longterm mitigation trajectories that reach atmospheric concentrations levels of about 450 to about $500 \mathrm{ppm} \mathrm{CO}_{2}$ eq by 2100 , but they do not preclude the option to meet that goal (robust evidence, high agreement). The Cancún Pledges are broadly consistent with cost-effective scenarios reaching about $550 \mathrm{ppm} \mathrm{CO}_{2}$ eq to $650 \mathrm{ppm} \mathrm{CO}_{2}$ eq by 2100 . Studies confirm that delaying mitigation through 2030 has a substantially larger influence on the subsequent challenges of mitigation than do delays through 2020 (Figures TS.9, TS.11). [6.4]

Only a limited number of studies have explored scenarios that are more likely than not to bring temperature change back to below $1.5^{\circ} \mathrm{C}$ by 2100 relative to pre-industrial levels; these scenarios bring atmospheric concentrations to below $430 \mathrm{ppm}$ $\mathrm{CO}_{2}$ eq by 2100 (high confidence). Assessing this goal is currently difficult because no multi-model study has explored these scenarios. The limited number of published studies exploring this goal have produced associated scenarios that are characterized by (1) immediate mitigation; (2) the rapid up-scaling of the full portfolio of mitigation technologies; and (3) development along a low-energy demand trajectory. ${ }^{12}$ $[6.3,7.11]$

\section{TS.3.1.3 Costs, investments and burden sharing}

Globally comprehensive and harmonized mitigation actions would result in significant economic benefits compared to fragmented approaches, but would require establishing effective institutions (high confidence). Economic analysis of mitigation scenarios demonstrates that globally comprehensive and harmonized mitigation actions achieve mitigation at least aggregate economic cost, since they allow mitigation to be undertaken where and when it is least expensive (see Box TS.7, Box TS.9). Most of these mitigation scenarios assume a global carbon price, which reaches all sectors of the economy. Instruments with limited coverage of GHG emissions reductions among sectors and climate policy regimes with fragmented regional

\footnotetext{
12 In these scenarios, the cumulative $\mathrm{CO}_{2}$ emissions range between $680-800 \mathrm{GtCO}_{2}$ for the period 2011-2050 and between $90-310 \mathrm{GtCO}_{2}$ for the period 2011-2100. Global $\mathrm{CO}_{2}$ eq emissions in 2050 are between $70-95 \%$ below 2010 emissions, and they are between 110-120\% below 2010 emissions in 2100 .
} 
$430-530 \mathrm{ppm} \mathrm{CO}$ eq in 2100

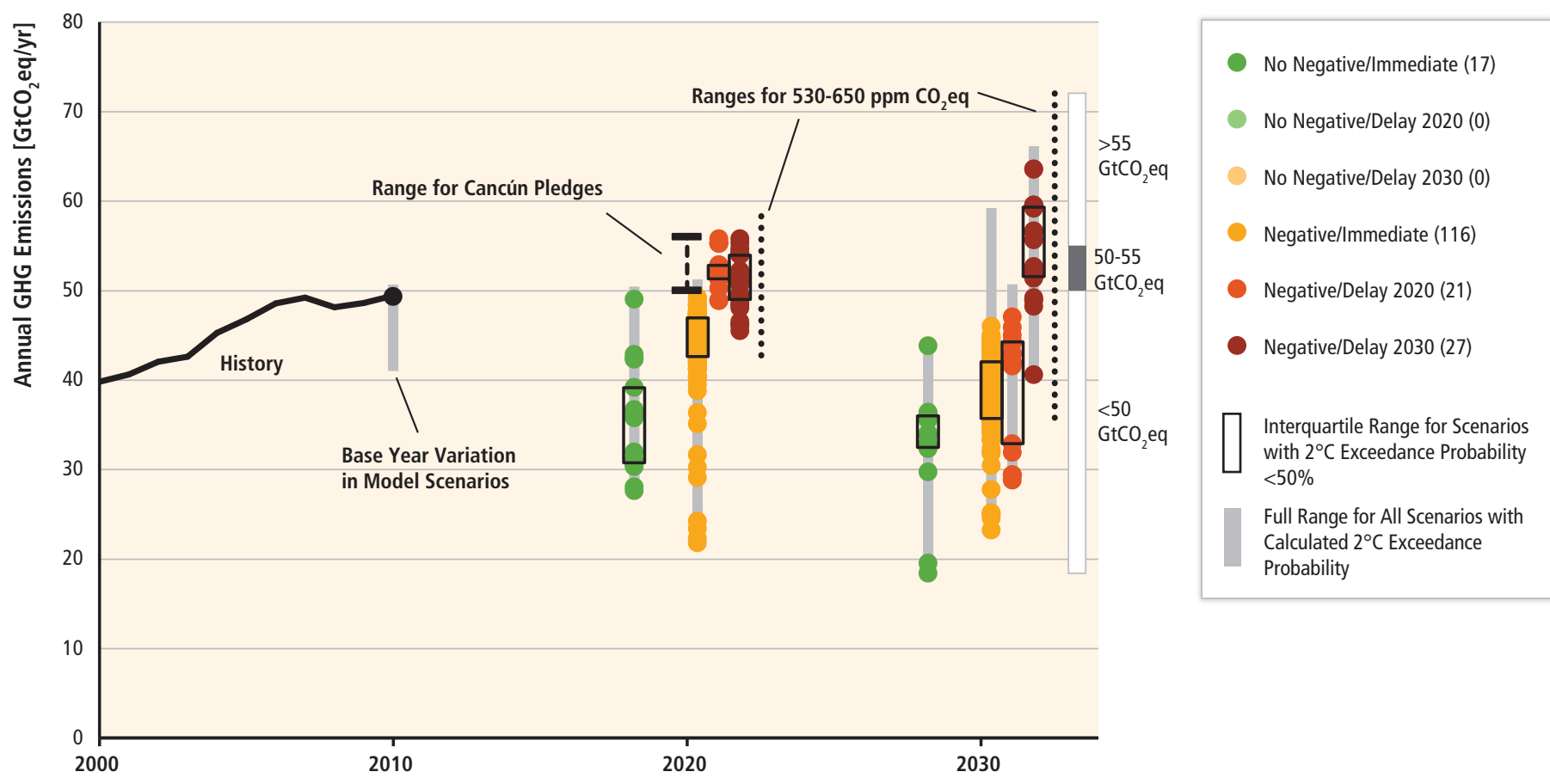

Figure TS.11| Near-term GHG emissions from mitigation scenarios reaching about 450 to about 500 (430-530) ppm $\mathrm{CO}_{2}$ eq concentrations by 2100 . The Figure includes only scenarios for which temperature exceedance probabilities were calculated. Individual model results are indicated with a data point when $2^{\circ} \mathrm{C}$ exceedance probability is below $50 \%$ as assessed by a simple carbon cycle/climate model (MAGICC). Colours refer to scenario classification in terms of whether net $\mathrm{CO}_{2}$ emissions become negative before 2100 (negative vs. no negative) and the timing of international participation in climate mitigation (immediate vs. delay until 2020 vs. delay until 2030). Number of reported individual results is shown in legend. The range of global GHG emissions in 2020 implied by the Cancún Pledges is based on analysis of alternative interpretations of national pledges. Note: In the WGIII AR5 scenario database, only four reported scenarios were produced based on delayed mitigation without net negative emissions while still lying below 530 ppm $\mathrm{CO}_{2}$ eq by 2100. They do not appear in the figure, because the model had insufficient coverage of non-gas species to enable a temperature calculation. Delay in these scenarios extended only to 2020, and their emissions fell in the same range as the 'No Negative/Immediate' category. Delay scenarios include both delayed global mitigation and fragmented action scenarios. [Figure 6.31, 13.13.1.3]

action increase aggregate economic costs. These cost increases are higher at more ambitious levels of mitigation. [6.3.6]

Estimates of the aggregate economic costs of mitigation vary widely, but increase with stringency of mitigation (high confidence). Most cost-effective scenarios collected for this assessment that are based on the assumptions that all countries of the world begin mitigation immediately, there is a single global carbon price applied to well-functioning markets, and key technologies are available, estimate that reaching about $450 \mathrm{ppm} \mathrm{CO}_{2}$ eq by 2100 would entail global consumption losses of $1 \%$ to $4 \%$ in 2030 (median: $1.7 \%$ ), $2 \%$ to $6 \%$ in 2050 (median: $3.4 \%$ ), and $3 \%$ to $11 \%$ in 2100 (median: $4.8 \%$ ) relative to consumption in baseline scenarios (those without additional mitigation efforts) that grows anywhere from $300 \%$ to more than $900 \%$ between 2010 and 2100 (baseline consumption growth represents the full range of corresponding baseline scenarios; Figure TS.12; Table TS.2 yellow segments). The consumption losses correspond to an annual average reduction of consumption growth by 0.06 to 0.2 percentage points from 2010 through 2030 (median: 0.09 ), 0.06 to 0.17 percentage points through 2050 (median: 0.09), and 0.04 to 0.14 percentage points over the century (median: 0.06 ). These numbers are relative to annual average consumption growth rates in baseline scenarios between $1.9 \%$ and $3.8 \%$ per year through 2050 and between $1.6 \%$ and $3 \%$ per year over the century (Table TS.2, yellow segments). These mitigation cost estimates do not consider the benefits of reduced climate change or co-benefits and adverse side-effects of mitigation (Box TS.9). Costs for maintaining concentrations in the range of $530-650 \mathrm{ppm} \mathrm{CO}_{2}$ eq are estimated to be roughly one-third to two-thirds lower than for associated $430-530 \mathrm{ppm} \mathrm{CO}_{2}$ eq scenarios. Cost estimates from scenarios can vary substantially across regions. Substantially higher cost estimates have been obtained based on assumptions about less idealized policy implementations and limits on technology availability as discussed below. Both higher and lower estimates have been obtained based on interactions with pre-existing distortions, non-climate market failures, or complementary policies. [6.3.6.2]

Delaying mitigation efforts beyond those in place today through 2030 or beyond could substantially increase mitigation costs in the decades that follow and the second half of the century (high confidence). Although delays in mitigation by any major emitter will reduce near-term mitigation costs, they will also result in more investment in carbon-intensive infrastructure and then rely on future 
Table TS.2 | Global mitigation costs in cost-effective scenarios ${ }^{1}$ and estimated cost increases due to assumed limited availability of specific technologies and delayed additional mitigation. Cost estimates shown in this table do not consider the benefits of reduced climate change as well as co-benefits and adverse side-effects of mitigation. The yellow columns show consumption losses (Figure TS.12, right panel) and annualized consumption growth reductions in cost-effective scenarios relative to a baseline development without climate policy. The grey columns show the percentage increase in discounted costs ${ }^{2}$ over the century, relative to cost-effective scenarios, in scenarios in which technology is constrained relative to default technology assumptions (Figure TS.13, left panel). ${ }^{3}$ The orange columns show the increase in mitigation costs over the periods 2030-2050 and 2050-2100, relative to scenarios with immediate mitigation, due to delayed additional mitigation through 2030 (see Figure TS.13, right panel). ${ }^{4}$ These scenarios with delayed additional mitigation are grouped by emission levels of less or more than $55 \mathrm{GtCO}_{2}$ eq in 2030, and two concentration ranges in 2100 (430-530 ppm $\mathrm{CO}_{2}$ eq and $530-650$ ppm $\mathrm{CO}_{2}$ eq). In all figures, the median of the scenario set is shown without parentheses, the range between the 16th and 84th percentile of the scenario set is shown in the parentheses, and the number of scenarios in the set is shown in square brackets. ${ }^{5}$ [Figures TS.12, TS.13, 6.21, 6.24, 6.25, Annex II.10]

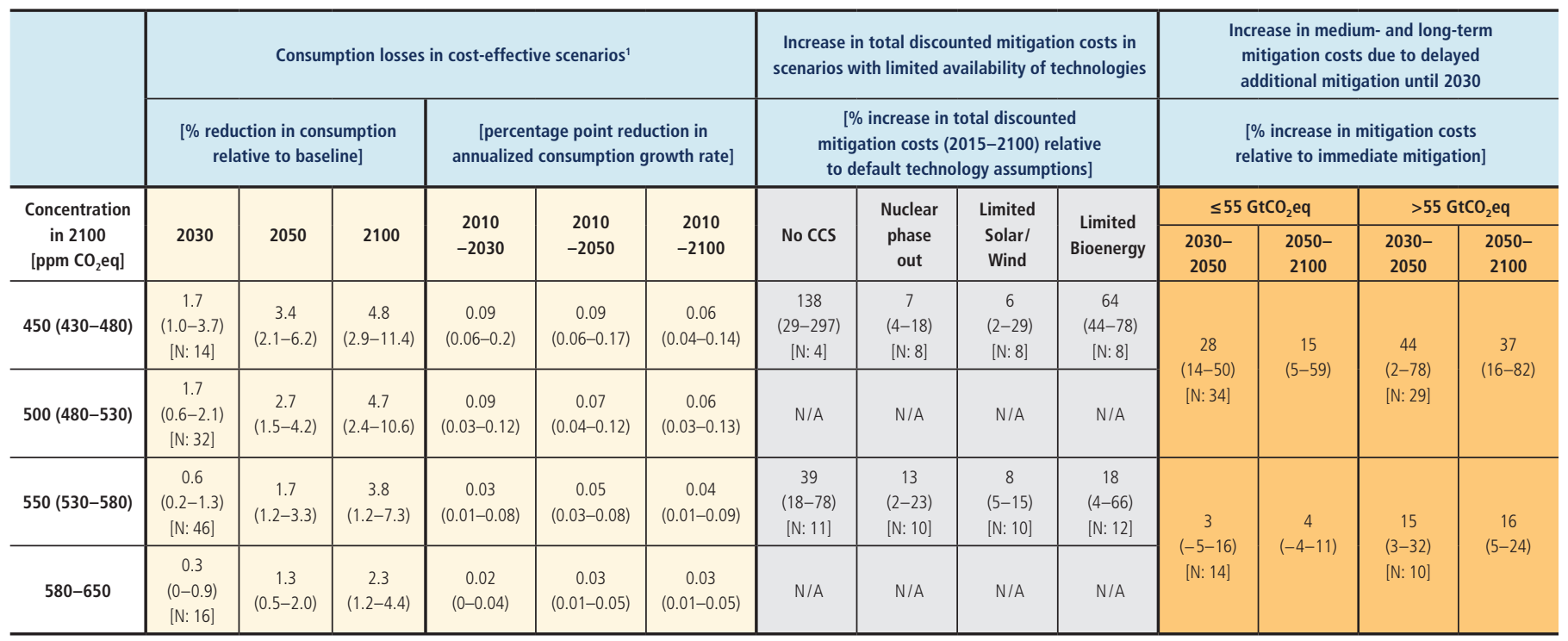

Notes:

Cost-effective scenarios assume immediate mitigation in all countries and a single global carbon price. In this analysis, they also impose no additional limitations on technology relative to the models' default technology assumptions.

Percentage increase of net present value of consumption losses in percent of baseline consumption (for scenarios from general equilibrium models) and abatement costs in percent of baseline GDP (for scenarios from partial equilibrium models) for the period 2015-2100, discounted (see Box TS.10) at $5 \%$ per year.

3 No CCS: CCS is not included in these scenarios. Nuclear phase out: No addition of nuclear power plants beyond those under construction, and operation of existing plants until the end of their lifetime. Limited Solar/Wind: a maximum of $20 \%$ global electricity generation from solar and wind power in any year of these scenarios. Limited Bioenergy: a maximum of $100 \mathrm{EJ} / \mathrm{yr}$ modern bioenergy supply globally (modern bioenergy used for heat, power, combinations, and industry was around $18 \mathrm{EJ} / \mathrm{yr}$ in 2008 [11.13.5]). 4 Percentage increase of total undiscounted mitigation costs for the periods 2030-2050 and 2050-2100.

The range is determined by the central scenarios encompassing the 16th and 84 th percentile of the scenario set. Only scenarios with a time horizon until 2100 are included. Some models that are included in the cost ranges for concentration levels above 530 ppm CO $\mathrm{CO}_{2}$ in 2100 could not produce associated scenarios for concentration levels below $530 \mathrm{ppm} \mathrm{CO}_{2}$ eq in 2100 with assumptions about limited availability of technologies and/or delayed additional mitigation (see caption of Figure TS.13 for more details).

decision makers to undertake a more rapid, deeper, and costlier future transformation of this infrastructure. Studies have found that aggregate costs, and associated carbon prices, rise more rapidly to higher levels in scenarios with delayed mitigation compared to scenarios where mitigation is undertaken immediately. Recent modelling studies have found that delayed mitigation through 2030 can substantially increase the aggregate costs of meeting 2100 concentrations of about 450 to about $500 \mathrm{ppm} \mathrm{CO}$ eq, particularly in scenarios with emissions greater than $55 \mathrm{GtCO}_{2}$ eq in 2030. (Figure TS.13, right panel; Table TS.2, orange segments) [6.3.6.4]

The technological options available for mitigation greatly influence mitigation costs and the challenges of reaching atmospheric concentration levels of about 450 to about $550 \mathrm{ppm}$ $\mathrm{CO}_{2}$ eq by 2100 (high confidence). Many models in recent model intercomparisons could not produce scenarios reaching atmospheric concentrations of about $450 \mathrm{ppm} \mathrm{CO}_{2}$ eq by 2100 with broadly pessimistic assumptions about key mitigation technologies. In these studies, the character and availability of CCS and bioenergy were found to have a particularly important influence on the mitigation costs and the challenges of reaching concentration levels in this range. For those models that could produce such scenarios, pessimistic assumptions about these increased discounted global mitigation costs of reaching concentration levels of about 450 and about $550 \mathrm{ppm} \mathrm{CO}_{2}$ eq by the end of the century significantly, with the effect being larger for more stringent mitigation scenarios (Figure TS.13, left panel; Table TS.2, grey segments). The studies also showed that reducing energy demand could potentially decrease mitigation costs significantly. [6.3.6.3]

The distribution of mitigation costs among different countries depends in part on the nature of effort-sharing frameworks and thus need not be the same as the distribution of mitigation efforts. Different effort-sharing frameworks draw upon different ethical principles (medium confidence). In cost-effective scenarios reaching concentrations of about 450 to about $550 \mathrm{ppm}$ $\mathrm{CO}_{2}$ eq in 2100 , the majority of mitigation investments over the course 

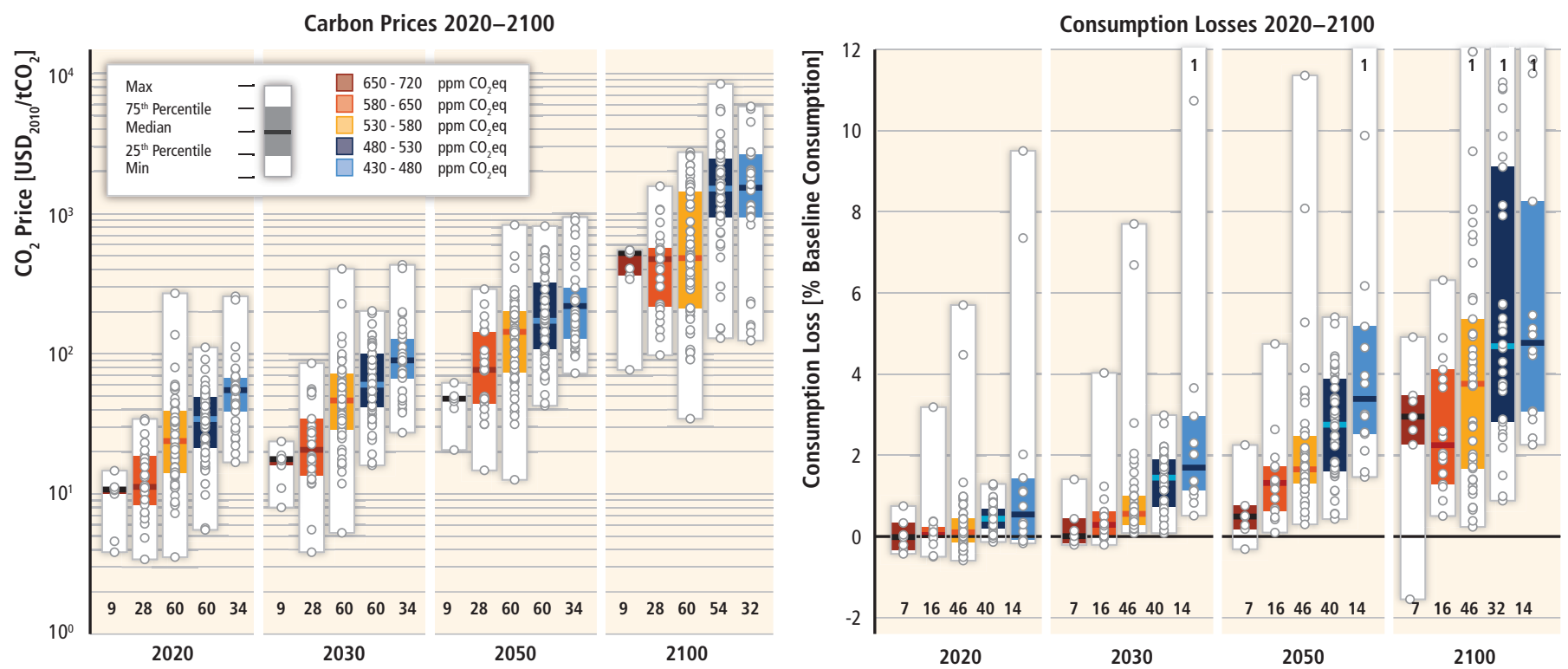

Figure TS.12 Global carbon prices (left panel) and consumption losses (right panel) over time in cost-effective, idealized implementation scenarios. Consumption losses are expressed as the percentage reduction from consumption in the baseline. The number of scenarios included in the boxplots is indicated at the bottom of the panels. The 2030 numbers also apply to 2020 and 2050. The number of scenarios outside the figure range is noted at the top. Note: The figure shows only scenarios that reported consumption losses (a subset of models with full coverage of the economy) or carbon prices, respectively, to 2050 or 2100 . Multiple scenarios from the same model with similar characteristics are only represented by a single scenario in the sample. [Figure 6.21]

\section{Box TS.9| The meaning of 'mitigation cost' in the context of mitigation scenarios}

Mitigation costs represent one component of the change in human welfare from climate change mitigation. Mitigation costs are expressed in monetary terms and generally are estimated against baseline scenarios, which typically involve continued, and sometimes substantial, economic growth and no additional and explicit mitigation efforts [3.9.3, 6.3.6]. Because mitigation cost estimates focus only on direct market effects, they do not take into account the welfare value (if any) of co-benefits or adverse side-effects of mitigation actions (Box TS.11) [3.6.3]. Further, these costs do not capture the benefits of reducing climate impacts through mitigation (Box TS.2).

There are a wide variety of metrics of aggregate mitigation costs used by economists, measured in different ways or at different places in the economy, including changes in GDP, consumption losses, equivalent variation and compensating variation, and loss in consumer and producer surplus. Consumption losses are often used as a metric because they emerge from many integrated models and they directly impact welfare. They can be expressed as a reduction in overall consumption relative to consumption in the corresponding baseline scenario in a given year or as a reduction of the average rate of consumption growth in the corresponding baseline scenario over a given time period.
Mitigation costs need to be distinguished from emissions prices. Emissions prices measure the cost of an additional unit of emissions reduction; that is, the marginal cost. In contrast, mitigation costs usually represent the total costs of all mitigation. In addition, emissions prices can interact with other policies and measures, such as regulatory policies directed at GHG reduction. If mitigation is achieved partly by these other measures, emissions prices may not reflect the actual costs of an additional unit of emissions reductions (depending on how additional emissions reductions are induced).

In general, estimates of global aggregate mitigation costs over the coming century from integrated models are based on largely stylized assumptions about both policy approaches and existing markets and policies, and these assumptions have an important influence on cost estimates. For example, cost-effective idealized implementation scenarios assume a uniform price on $\mathrm{CO}_{2}$ and other GHGs in every country and sector across the globe, and constitute the least cost approach in the idealized case of largely efficient markets without market failures other than the climate change externality. Most long-term, global scenarios do not account for the interactions between mitigation and pre-existing or new policies, market failures, and distortions. Climate policies can interact with existing policies to increase or reduce the actual cost of climate policies. [3.6.3.3, 6.3.6.5] 

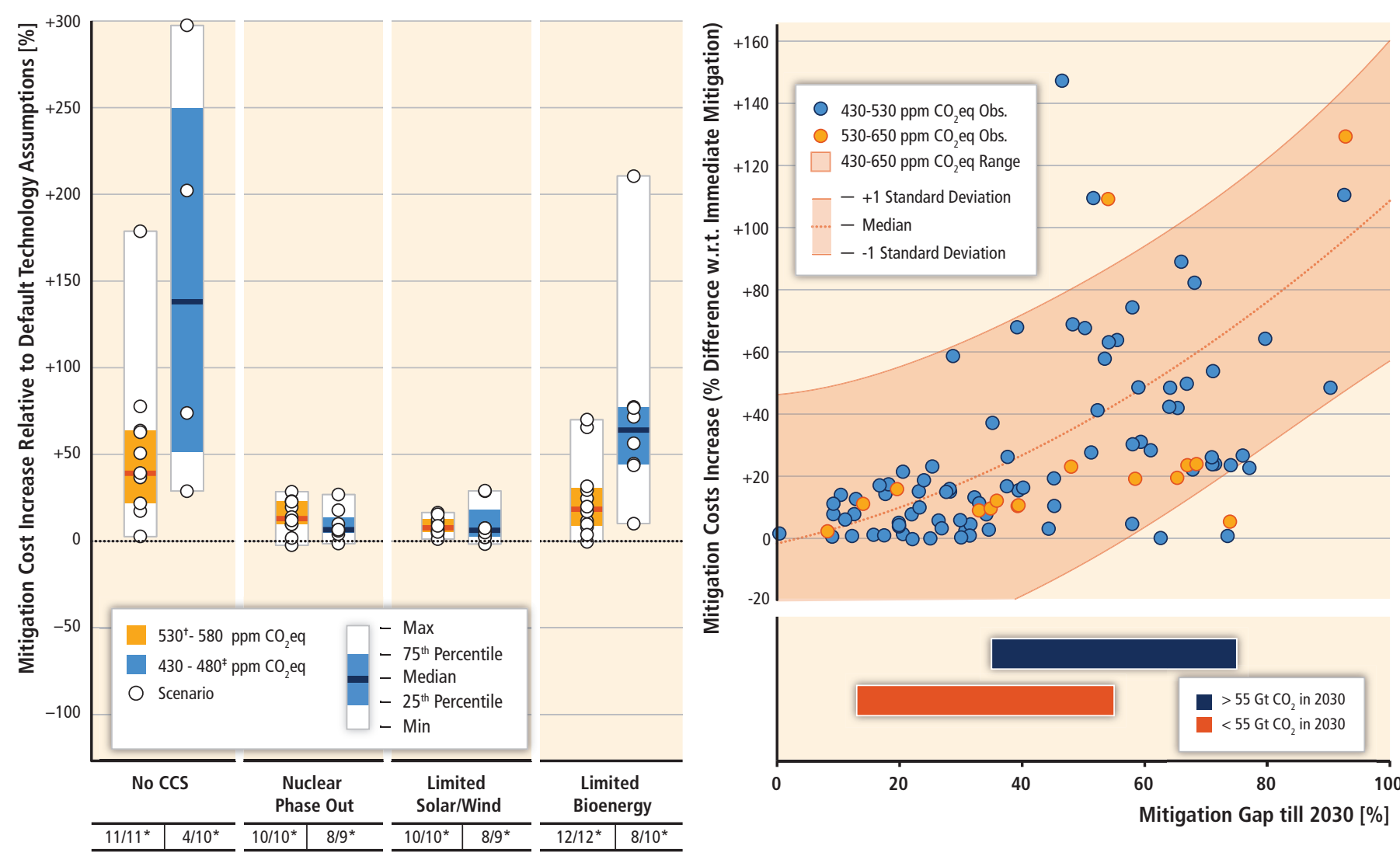

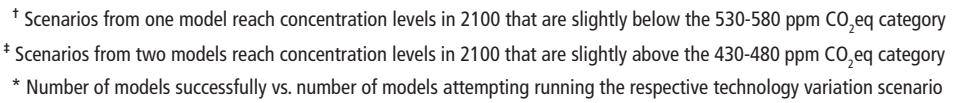

Figure TS.13 Left panel shows the relative increase in net present value mitigation costs (2015-2100, discounted at $5 \%$ per year) from technology portfolio variations relative to a scenario with default technology assumptions. Scenario names on the horizontal axis indicate the technology variation relative to the default assumptions: No CCS = unavailability of carbon dioxide capture and storage (CCS); Nuclear phase out = No addition of nuclear power plants beyond those under construction; existing plants operated until the end of their lifetime; Limited Solar/Wind = a maximum of $20 \%$ global electricity generation from solar and wind power in any year of these scenarios; Limited Bioenergy = a maximum of 100 exajoules per year (EJ/yr) modern bioenergy supply globally. [Figure 6.24] Right panel shows increase in long-term mitigation costs for the period 2050-2100 (sum over undiscounted costs) as a function of reduced near-term mitigation effort, expressed as the relative change between scenarios implementing mitigation immediately and those that correspond to delayed additional mitigation through 2020 or 2030 (referred to here as 'mitigation gap'). The mitigation gap is defined as the difference in cumulative $\mathrm{CO}_{2}$ emissions reductions until 2030 between the immediate and delayed additional mitigation scenarios. The bars in the lower right panel indicate the mitigation gap range where $75 \%$ of scenarios with 2030 emissions above (dark blue) and below (red) $55 \mathrm{GtCO}_{2}$, respectively, are found. Not all model simulations of delayed additional mitigation until 2030 could reach the lower concentration goals of about 450 or $500(430-530)$ ppm CO 2 eq (for 2030 emissions above $55 \mathrm{GtCO}_{2}$ eq, 29 of 48 attempted simulations could reach the goal; for 2030 emissions below $55 \mathrm{GtCO}_{2}$ eq, 34 of 51 attempted simulations could reach the goal). [Figure 6.25]

of century occur in the non-OECD countries. Some studies exploring particular effort-sharing frameworks, under the assumption of a global carbon market, estimate that the associated financial flows could be in the order of hundred billions of USD per year before mid-century to bring concentrations to between about 450 and about $500 \mathrm{ppm} \mathrm{CO}_{2} \mathrm{eq}$ in 2100. Most studies assume efficient mechanisms for international carbon markets, in which case economic theory and empirical research suggest that the choice of effort sharing allocations will not meaningfully affect the globally efficient levels of regional abatement or aggregate global costs. Actual approaches to effort-sharing can deviate from this assumption. $[3.3,6.3 .6 .6,13.4 .2 .4]$

Geoengineering denotes two clusters of technologies that are quite distinct: carbon dioxide removal (CDR) and solar radiation management (SRM). Mitigation scenarios assessed in AR5 do not assume any geoengineering options beyond large-scale CDR due to afforestation and BECCS. CDR techniques include afforestation, using bioenergy along with CCS (BECCS), and enhancing uptake of $\mathrm{CO}_{2}$ by the oceans through iron fertilization or increasing alkalinity. Most terrestrial CDR techniques would require large-scale land-use changes and could involve local and regional risks, while maritime CDR may involve significant transboundary risks for ocean ecosystems, so that its deployment could pose additional challenges for cooperation between countries. With currently known technologies, CDR could not be deployed quickly on a large scale. SRM includes various technologies to offset crudely some of the climatic effects of the build-up of GHGs in the atmosphere. It works by adjusting the planet's heat balance through a small increase in the reflection of incoming sunlight such as by injecting particles or aerosol precursors in the upper atmosphere. SRM has attracted considerable attention, mainly 


\section{Box TS.10 | Future goods should be discounted at an appropriate rate}

Investments aimed at mitigating climate change will bear fruit far in the future, much of it more than 100 years from now. To decide whether a particular investment is worthwhile, its future benefits need to be weighed against its present costs. In doing this, economists do not normally take a quantity of commodities at one time as equal in value to the same quantity of the same commodities at a different time. They normally give less value to later commodities than to earlier ones. They 'discount' later commodities, that is to say. The rate at which the weight given to future goods diminishes through time is known as the 'discount rate' on commodities.

There are two types of discount rates used for different purposes. The market discount rate reflects the preferences of presently living people between present and future commodities. The social discount rate is used by society to compare benefits of present members of society with those not yet born. Because living people may be impatient, and because future people do not trade in the market, the market may not accurately reflect the value of commodities that will come to future people relative to those that come to present people. So the social discount rate may differ from the market rate.

The chief reason for social discounting (favouring present people over future people) is that commodities have 'diminishing marginal benefit' and per capita income is expected to increase over time. Diminishing marginal benefit means that the value of extra commodities to society declines as people become better off. If economies continue to grow, people who live later in time will on average be better off-possess more commodities - than people who live earlier. The faster the growth and the greater the degree of diminishing marginal benefit, the greater should be the discount rate on commodities. If per capita growth is expected to be negative (as it is in some countries), the social discount rate may be negative.

Some authors have argued, in addition, that the present generation of people should give less weight to later people's well-being just because they are more remote in time. This factor would add to the social discount rate on commodities.

The social discount rate is appropriate for evaluating mitigation projects that are financed by reducing current consumption. If a project is financed partly by 'crowding out' other investments, the benefits of those other investments are lost, and their loss must be counted as an opportunity cost of the mitigation project. If a mitigation project crowds out an exactly equal amount of other investment, then the only issue is whether or not the mitigation investment produces a greater return than the crowded-out investment. This can be tested by evaluating the mitigation investment using a discount rate equal to the return that would have been expected from the crowded out investment. If the market functions well, this will be the market discount rate. [3.6.2] because of the potential for rapid deployment in case of climate emergency. The suggestion that deployment costs for individual technologies could potentially be low could result in new challenges for international cooperation because nations may be tempted to prematurely deploy unilaterally systems that are perceived to be inexpensive. Consequently, SRM technologies raise questions about costs, risks, governance, and ethical implications of developing and deploying SRM, with special challenges emerging for international institutions, norms and other mechanisms that could coordinate research and restrain testing and deployment. [1.4, 3.3.7, 6.9, 13.4.4]

Knowledge about the possible beneficial or harmful effects of SRM is highly preliminary. SRM would have varying impacts on regional climate variables such as temperature and precipitation, and might result in substantial changes in the global hydrological cycle with uncertain regional effects, for example on monsoon precipitation. Non-climate effects could include possible depletion of stratospheric ozone by stratospheric aerosol injections. A few studies have begun to examine climate and non-climate impacts of SRM, but there is very little agreement in the scientific community on the results or on whether the lack of knowledge requires additional research or eventually field testing of SRM-related technologies. [1.4, 3.3.7, 6.9, 13.4.4]

\section{TS.3.1.4 Implications of mitigation pathways for other objectives}

Mitigation scenarios reaching about 450 to about $500 \mathrm{ppm}$ $\mathrm{CO}_{2}$ eq by 2100 show reduced costs for achieving energy security and air quality objectives (medium confidence) (Figure TS.14, lower panel). The mitigation costs of most of the scenarios in this assessment do not consider the economic implications of the cost reductions for these other objectives (Box TS.9). There is a wide range of co-benefits and adverse side-effects other than air quality and energy security (Tables TS.4-8). The impact of mitigation on the overall costs for achieving many of these other objectives as well as the associated welfare implications are less well understood and have not been assessed thoroughly in the literature (Box TS.11). [3.6.3, $4.8,6.6]$ 


\section{Co-Benefits of Climate Change Mitigation for Energy Security and Air Quality}

LIMITS Model Inter-Comparison

Impact of Climate Policy on Energy Security

Energy Trade

(Global, 2050)

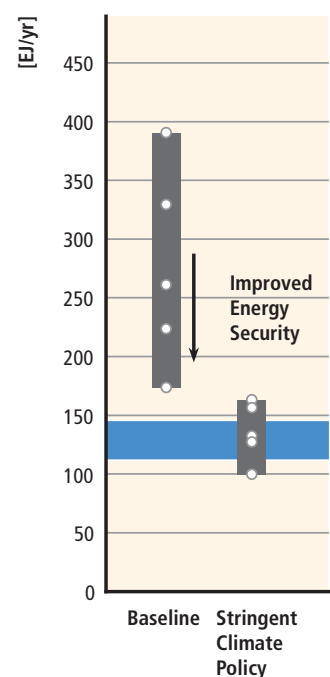

Energy Security Levels of GEA Scenarios in Bottom Panel
IPCC AR5 Scenario Ensemble

Impact of Climate Policy on Air Pollutant Emissions (Global, 2005-2050)
Cumulative Oil Extraction Electricity Diversity (Global, 2010-2050) (Global, 2050)
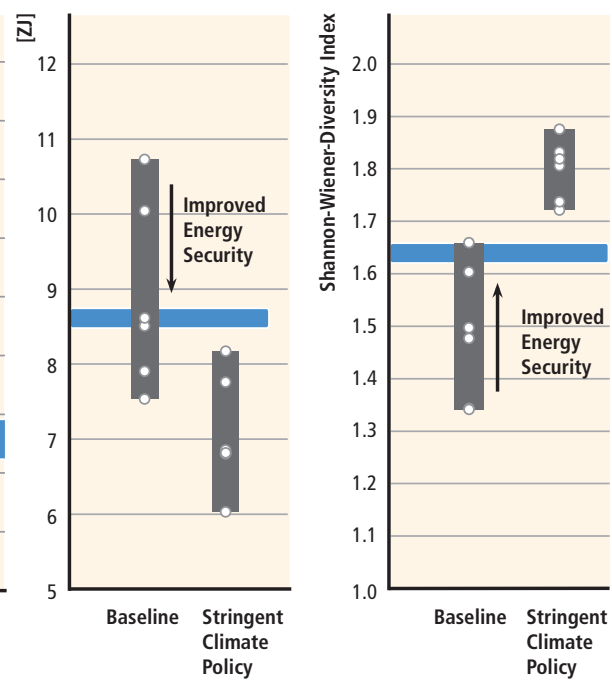

Policy

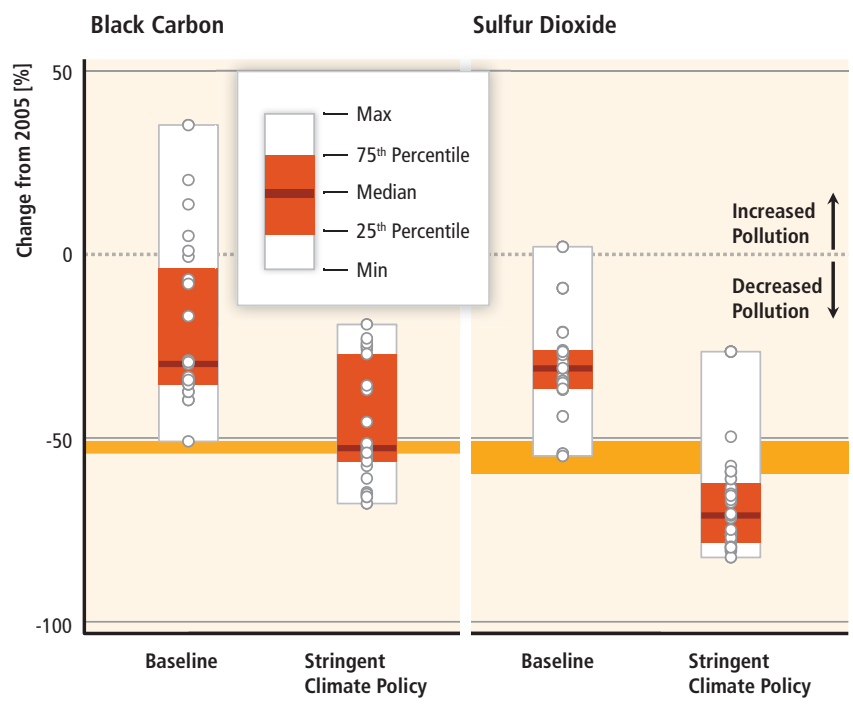

Air Quality Levels of GEA Scenarios in Bottom Panel

\section{Policy Costs of Achieving Different Objectives}

Global Energy Assessment Scenario Ensemble $(n=624)$

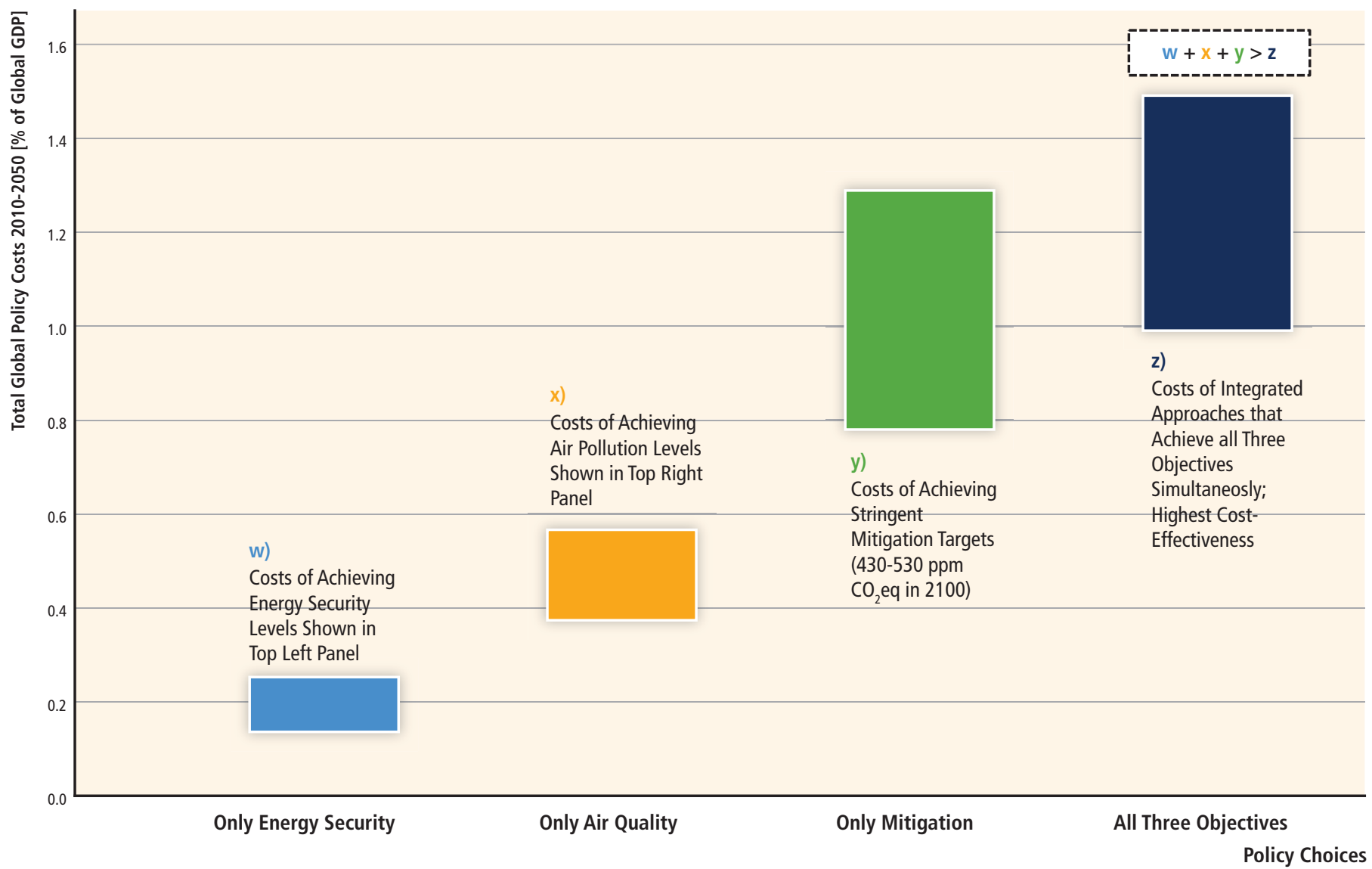


Figure TS.14| Co-benefits of mitigation for energy security and air quality in scenarios with stringent climate policies reaching about 450 to about 500 (430-530) ppm CO ${ }_{2}$ eq concentrations in 2100. Upper panels show co-benefits for different security indicators and air pollutant emissions. Lower panel shows related global policy costs of achieving the energy security, air quality, and mitigation objectives, either alone $(w, x, y)$ or simultaneously $(z)$. Integrated approaches that achieve these objectives simultaneously show the highest cost-effectiveness due to synergies $(w+x+y>z)$. Policy costs are given as the increase in total energy system costs relative to a baseline scenario without additional efforts to reduce GHG emissions beyond those in place today. Costs are indicative and do not represent full uncertainty ranges. [Figure 6.33]

Mitigation scenarios reaching about 450 to about $500 \mathrm{ppm}$ $\mathrm{CO}_{2}$ eq by 2100 show co-benefits for energy security objectives, enhancing the sufficiency of resources to meet national energy demand as well as the resilience of the energy system (medium confidence). These mitigation scenarios show improvements in terms of the diversity of energy sources and reduction of energy imports, resulting in energy systems that are less vulnerable to price volatility and supply disruptions (Figure TS.14, upper left panel). [6.3.6, 6.6, 7.9, 8.7, 9.7, 10.8, 11.13.6, 12.8]

Mitigation policy could devalue fossil fuel assets and reduce revenues for fossil fuel exporters, but differences between regions and fuels exist (high confidence). Most mitigation scenarios are associated with reduced revenues from coal and oil trade for major exporters (high confidence). However, a limited number of studies find that mitigation policies could increase the relative competitiveness of conventional oil vis-à-vis more carbon-intensive unconventional oil and 'coal-to-liquids'. The effect of mitigation on natural gas export revenues is more uncertain, with some studies showing possible benefits for export revenues in the medium term until about 2050 (medium confidence). The availability of CCS would reduce the adverse effect of mitigation on the value of fossil fuel assets (medium confidence). $[6.3 .6,6.6,14.4 .2]$

Fragmented mitigation policy can provide incentives for emission-intensive economic activity to migrate away from a region that undertakes mitigation (medium confidence). Scenario studies have shown that such 'carbon leakage' rates of energy-related emissions are relatively contained, often below $20 \%$ of the emissions reductions. Leakage in land-use emissions could be substantial, though fewer studies have quantified it. While border tax adjustments are seen as enhancing the competitiveness of GHG- and trade-intensive industries within a climate policy regime, they can also entail welfare losses for non-participating, and particularly developing, countries. $[5.4,6.3,13.8,14.4]$

Mitigation scenarios leading to atmospheric concentration levels of about 450 to about $500 \mathrm{ppm} \mathrm{CO}_{2} \mathrm{eq}$ in 2100 are associated with significant co-benefits for air quality and related human health and ecosystem impacts. The benefits from major cuts in air pollutant emissions are particularly high where currently legislated and planned air pollution controls are weak (high confidence). Stringent mitigation policies result in co-controls with major cuts in air pollutant emissions significantly below baseline scenarios (Figure TS.14, upper right panel). Co-benefits for health are particularly high in today's developing world. The extent to which air pollution policies, targeting for example black carbon (BC), can mitigate climate change is uncertain. $[5.7,6.3,6.6,7.9,8.7,9.7,10.8,11.7,11.13 .6$, 12.8; WGII 11.9]

There is a wide range of possible adverse side-effects as well as co-benefits and spillovers from climate policy that have not been well-quantified (high confidence). Whether or not side-effects materialize, and to what extent side-effects materialize, will be caseand site-specific, as they will depend on local circumstances and the scale, scope, and pace of implementation. Important examples include biodiversity conservation, water availability, food security, income distribution, efficiency of the taxation system, labour supply and employment, urban sprawl, and the sustainability of the growth of developing countries. (Box TS.11)

Some mitigation policies raise the prices for some energy services and could hamper the ability of societies to expand access to modern energy services to underserved populations (low confidence). These potential adverse side-effects can be avoided with the adoption of complementary policies (medium confidence). Most notably, about 1.3 billion people worldwide do not have access to electricity and about 3 billion are dependent on traditional solid fuels for cooking and heating with severe adverse effects on health, ecosystems and development. Providing access to modern energy services is an important sustainable development objective. The costs of achieving nearly universal access to electricity and clean fuels for cooking and heating are projected to be between 72 to 95 billion USD per year until 2030 with minimal effects on GHG emissions (limited evidence, medium agreement). A transition away from the use of traditional biomass ${ }^{13}$ and the more efficient combustion of solid fuels reduce air pollutant emissions, such as sulfur dioxide $\left(\mathrm{SO}_{2}\right)$, nitrogen oxides $\left(\mathrm{NO}_{\mathrm{x}}\right)$, carbon monoxide $(\mathrm{CO})$, and black carbon $(\mathrm{BC})$, and thus yield large health benefits (high confidence). [4.3, 6.6, 7.9, $9.3,9.7,11.13 .6,16.8]$

The effect of mitigation on water use depends on technological choices and the portfolio of mitigation measures (high confidence). While the switch from fossil energy to renewable energy like photovoltaic (PV) or wind can help reducing water use of the energy system, deployment of other renewables, such as some forms of hydropower, concentrated solar power (CSP), and bioenergy may have adverse effects on water use. [6.6, 7.9, 9.7, 10.8, 11.7, 11.13.6]

\footnotetext{
Traditional biomass refers to the biomass - fuelwood, charcoal, agricultural residues, and animal dung - used with the so-called traditional technologies such as open fires for cooking, rustic kilns and ovens for small industries (see Glossary).
} 


\section{Box TS.11 | Accounting for the co-benefits and adverse side-effects of mitigation}

A government policy or a measure intended to achieve one objective (such as mitigation) will also affect other objectives (such as local air quality). To the extent these side-effects are positive, they can be deemed 'co-benefits'; otherwise they are termed 'adverse side-effects'. In this report, co-benefits and adverse side-effects are measured in non-monetary units. Determining the value of these effects to society is a separate issue. The effects of co-benefits on social welfare are not evaluated in most studies, and one reason is that the value of a co-benefit depends on local circumstances and can be positive, zero, or even negative. For example, the value of the extra tonne of sulfur dioxide $\left(\mathrm{SO}_{2}\right)$ reduction that occurs with mitigation depends greatly on the stringency of existing $\mathrm{SO}_{2}$ control policies: in the case of weak existing $\mathrm{SO}_{2}$ policy, the value of $\mathrm{SO}_{2}$ reductions may be large, but in the case of stringent existing $\mathrm{SO}_{2}$ policy it may be near zero. If $\mathrm{SO}_{2}$ policy is too stringent, the value of the co-benefit may be negative (assuming $\mathrm{SO}_{2}$ policy is not adjusted). While climate policy affects non-climate objectives (Tables TS.4-8) other policies also affect climate change outcomes. [3.6.3, 4.8, 6.6, Glossary]

Mitigation can have many potential co-benefits and adverse side-effects, which makes comprehensive analysis difficult. The direct benefits of climate policy include, for example, intended effects on global mean surface temperature, sea level rise, agricultural productivity, biodiversity, and health effects of global warming [WGII TS]. The co-benefits and adverse side-effects of climate policy could include effects on a partly overlapping set of objectives such as local air pollutant emissions reductions and related health and ecosystem impacts, biodiversity conservation, water availability, energy and food security, energy access, income distribution, efficiency of the taxation system, labour supply and employment, urban sprawl, and the sustainability of the growth of developing countries $[3.6,4.8,6.6$, 15.2].

All these side-effects are important, because a comprehensive evaluation of climate policy needs to account for benefits and costs related to other objectives. If overall social welfare is to be determined and quantified, this would require valuation methods and a consideration of pre-existing efforts to attain the many objectives. Valuation is made difficult by factors such as interaction between climate policies and pre-existing nonclimate policies, externalities, and non-competitive behaviour. [3.6.3]
Mitigation scenarios and sectoral studies show that overall the potential for co-benefits of energy end-use measures outweigh the potential adverse side-effects, whereas the evidence suggests this may not be the case for all energy supply and AFOLU measures (high confidence). (Tables TS.4-8) [4.8, 5.7, 6.6, 7.9, 8.7, $9.7,10.8,11.7,11.13 .6,12.8]$

\section{TS.3.2 Sectoral and cross-sectoral mitigation measures}

Anthropogenic GHG emissions result from a broad set of human activities, most notably those associated with energy supply and consumption and with the use of land for food production and other purposes. A large proportion of emissions arise in urban areas. Mitigation options can be grouped into three broad sectors: (1) energy supply, (2) energy end-use sectors including transport, buildings, industry, and (3) AFOLU. Emissions from human settlements and infrastructures cut across these different sectors. Many mitigation options are linked. The precise set of mitigation actions taken in any sector will depend on a wide range of factors, including their relative economics, policy structures, normative values, and linkages to other policy objectives. The first section examines issues that cut across the sectors and the following subsections examine the sectors themselves.

\section{TS.3.2.1 Cross-sectoral mitigation pathways and measures}

Without new mitigation policies GHG emissions are projected to grow in all sectors, except for net $\mathrm{CO}_{2}$ emissions in the AFOLU $^{14}$ sector (robust evidence, medium agreement). Energy supply sector emissions are expected to continue to be the major source of GHG emissions in baseline scenarios, ultimately accounting for the significant increases in indirect emissions from electricity use in the buildings and the industry sectors. Deforestation decreases in most of the baseline scenarios, which leads to a decline in net $\mathrm{CO}_{2}$ emissions from the AFOLU sector. In some scenarios the AFOLU sector changes from an emission source to a net emission sink towards the end of the century. (Figure TS.15) [6.3.1.4, 6.8]

Infrastructure developments and long-lived products that lock societies into GHG-intensive emissions pathways may be difficult or very costly to change, reinforcing the importance of early action for ambitious mitigation (robust evidence, high agreement). This lock-in risk is compounded by the lifetime of the infrastructure, by the difference in emissions associated with alternatives, and

\footnotetext{
14 Net AFOLU $\mathrm{CO}_{2}$ emissions include emissions and removals of $\mathrm{CO}_{2}$ from the AFOLU sector, including land under forestry and, in some assessments, $\mathrm{CO}_{2}$ sinks in agricultural soils.
} 
the magnitude of the investment cost. As a result, lock-in related to infrastructure and spatial planning is the most difficult to eliminate, and thus avoiding options that lock high emission patterns in permanently is an important part of mitigation strategies in regions with rapidly developing infrastructure. In mature or established cities, options are constrained by existing urban forms and infrastructure, and limits on the potential for refurbishing or altering them. However, materials, products and infrastructure with long lifetimes and low lifecycle emissions can ensure positive lock-in as well as avoid emissions through dematerialization (i.e., through reducing the total material inputs required to deliver a final service). [5.6.3, 6.3.6.4, 9.4, 10.4, 12.3, 12.4]

Systemic and cross-sectoral approaches to mitigation are expected to be more cost-effective and more effective in cutting emissions than sector-by-sector policies (medium confidence). Cost-effective mitigation policies need to employ a system perspective in order to account for inter-dependencies among different economic sectors and to maximize synergistic effects. Stabilizing atmospheric $\mathrm{CO}_{2}$ eq concentrations at any level will ultimately require deep reductions in emissions and fundamental changes to both the end-use and supply-side of the energy system as well as changes in land-use practices and industrial processes. In addition, many low-carbon energy supply technologies (including (CS) and their infrastructural requirements face public acceptance issues limiting their deployment. This applies also to the adoption of new technologies, and structural and behavioural change, in the energy enduse sectors (robust evidence, high agreement) [7.9.4, 8.7, 9.3.10, $9.8,10.8,11.3,11.13]$. Lack of acceptance may have implications not only for mitigation in that particular sector, but also for wider mitigation efforts.

Integrated models identify three categories of energy system related mitigation measures: the decarbonization of the energy supply sector, final energy demand reductions, and the switch to low-carbon energy carriers, including electricity, in the energy end-use sectors (robust evidence, high agreement) [6.3.4, 6.8, 7.11]. The broad range of sectoral mitigation options available mainly relate to achieving reductions in GHG emissions intensity, energy intensity and changes in activity (Table TS.3) [7.5, 8.3, 8.4, 9.3, 10.4, 12.4]. Direct options in AFOLU involve storing carbon in terrestrial systems (for example, through afforestation) and providing bioenergy feedstocks $[11.3,11.13]$. Options to reduce non- $\mathrm{CO}_{2}$ GHG emissions exist across all sectors, but most notably in agriculture, energy supply, and industry.

Demand reductions in the energy end-use sectors, due to, e.g., efficiency enhancement and behavioural change, are a key miti-
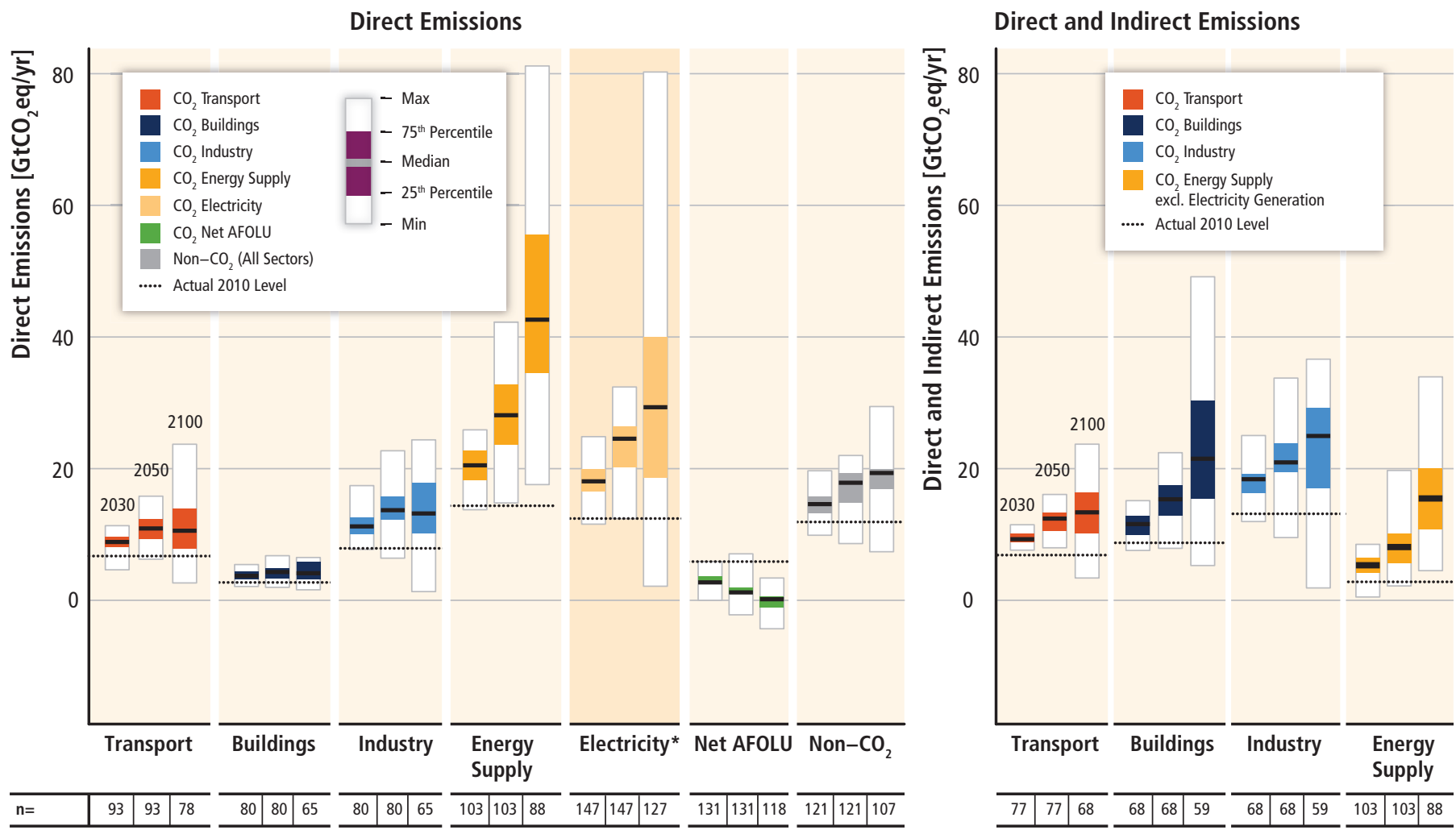

Figure TS.15| Direct (left panel) and direct and indirect emissions (right panel) of $\mathrm{CO}_{2}$ and non- $\mathrm{CO}_{2} \mathrm{GHG}$ across sectors in baseline scenarios. Non- $\mathrm{CO}_{2} \mathrm{GHGs}_{\text {are }}$ converted to $\mathrm{CO}_{2}$-equivalents based on Global Warming Potentials with a 100-year time horizon from the IPCC Second Assessment Report (SAR) (see Box TS.5). Note that in the case of indirect emissions, only electricity generation emissions are allocated from energy supply to end-use sectors. In the left panel electricity sector emissions are shown (Electricity ${ }^{*}$ ) in addition to energy supply sector emissions which they are part of, to illustrate their large role on the energy supply side. The numbers at the bottom refer to the number of scenarios included in the ranges that differ across sectors and time due to different sectoral resolutions and time horizons of models. [Figure 6.34] 
Liquids and Hydrogen

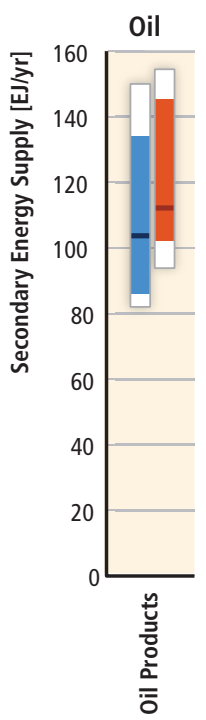

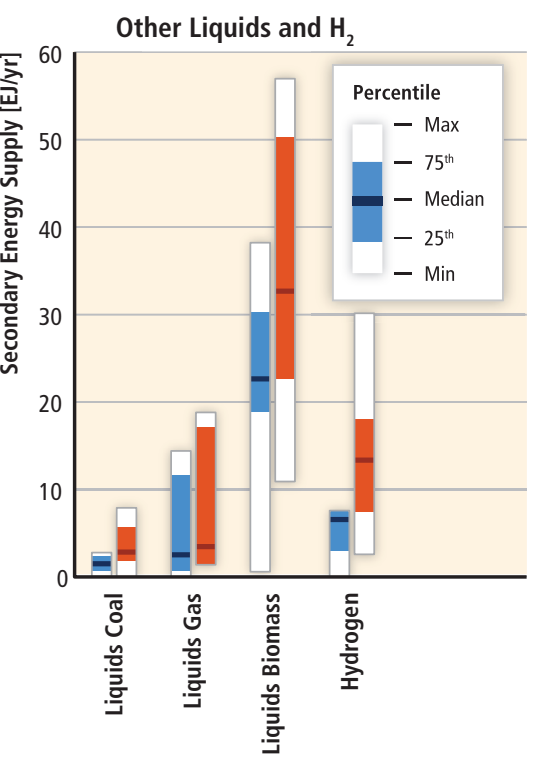

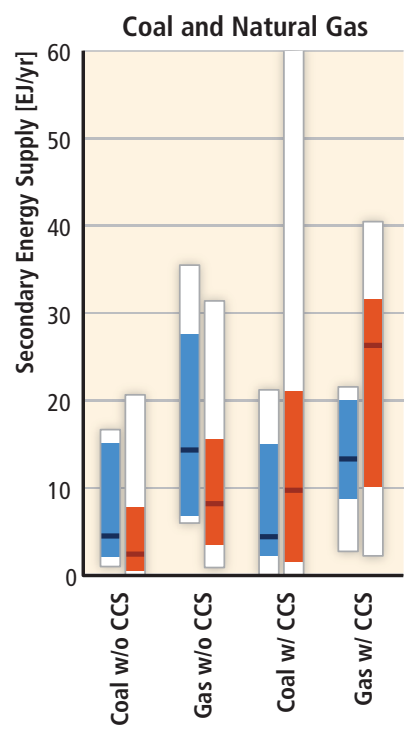

Electricity Generation

\begin{tabular}{l|l|l|l}
\hline 1 & 2 & 3 & 4 \\
\hline $\begin{array}{l}\text { High energy } \\
\text { demand scenarios } \\
\text { show higher levels } \\
\text { of oil supply. }\end{array}$ & $\begin{array}{l}\text { In high energy demand scenarios, alternative liquid } \\
\text { and hydrogen technologies are scaled up more } \\
\text { rapidly. }\end{array}$ & $\begin{array}{l}\text { High energy demand scenarios show } \\
\text { a more rapid up-scaling of CCS } \\
\text { technologies but a more rapid phase- } \\
\text { out of unabated fossil fuel conversion } \\
\text { technologies. }\end{array}$ & $\begin{array}{l}\text { In high energy demand scenarios non-fossil electricity } \\
\text { generation technologies are scaled up more rapidly. }\end{array}$ \\
\hline
\end{tabular}

Figure TS.16| Influence of energy demand on the deployment of energy supply technologies in 2050 in mitigation scenarios reaching about 450 to about 500 (430-530) ppm $\mathrm{CO}_{2}$ eq concentrations by 2100 . Blue bars for 'low energy demand' show the deployment range of scenarios with limited growth of final energy of < $20 \%$ in 2050 compared to 2010. Red bars show the deployment range of technologies in case of 'high energy demand' (> $20 \%$ growth in 2050 compared to 2010). For each technology, the median, interquartile, and full deployment range is displayed. Notes: Scenarios assuming technology restrictions and scenarios with final energy in the base-year outside $\pm 5 \%$ of 2010 inventories are excluded. Ranges include results from many different integrated models. Multiple scenario results from the same model were averaged to avoid sampling biases; see Chapter 6 for further details. [Figure 7.11]

gation strategy and affect the scale of the mitigation challenge for the energy supply side (high confidence). Limiting energy demand: (1) increases policy choices by maintaining flexibility in the technology portfolio; (2) reduces the required pace for up-scaling low-carbon energy supply technologies and hedges against related supply-side risks (Figure TS.16); (3) avoids lock-in to new, or potentially premature retirement of, carbon-intensive infrastructures; (4) maximizes co-benefits for other policy objectives, since the potential for co-benefits of energy end-use measures outweighs the potential for adverse side-effects which may not be the case for all supply-side measures (see Tables TS.4-8); and (5) increases the cost-effectiveness of the transformation (as compared to mitigation strategies with higher levels of energy demand) (medium confidence). However, energy service demand reductions are unlikely in developing countries or for poorer population segments whose energy service levels are low or partially unmet. $[6.3 .4,6.6,7.11,10.4]$

Behaviour, lifestyle, and culture have a considerable influence on energy use and associated emissions, with a high mitigation potential in some sectors, in particular when complementing technological and structural change (medium evidence, medium agreement). Emissions can be substantially lowered through: changes in consumption patterns (e. g., mobility demand and mode, energy use in households, choice of longer-lasting products); dietary change and reduction in food wastes; and change of lifestyle (e. g., stabilizing/lowering consumption in some of the most developed countries, sharing economy and other behavioural changes affecting activity) (Table TS.3). [8.1, 8.9, 9.2, 9.3, Box 10.2, 10.4, 11.4, 12.4, 12.6, 12.7]

Evidence from mitigation scenarios indicates that the decarbonization of energy supply is a key requirement for stabilizing atmospheric $\mathrm{CO}_{2}$ eq concentrations below $580 \mathrm{ppm}$ (robust evidence, high agreement). In most long-term mitigation scenarios not exceeding $580 \mathrm{ppm} \mathrm{CO}_{2}$ eq by 2100 , global energy supply is fully decarbonized at the end of the 21st century with many scenarios relying on a net removal of $\mathrm{CO}_{2}$ from the atmosphere. However, because existing supply systems are largely reliant on carbon-intensive fossil fuels, energy intensity reductions can equal or outweigh decarbonization of energy supply in the near term. In the buildings and industry sector, for example, efficiency improvements are an important strategy for reducing indirect emissions from electricity generation (Figure TS.15). In the long term, the reduction in electricity generation emissions is accompanied by an increase in the share of electricity in end uses (e.g., for 

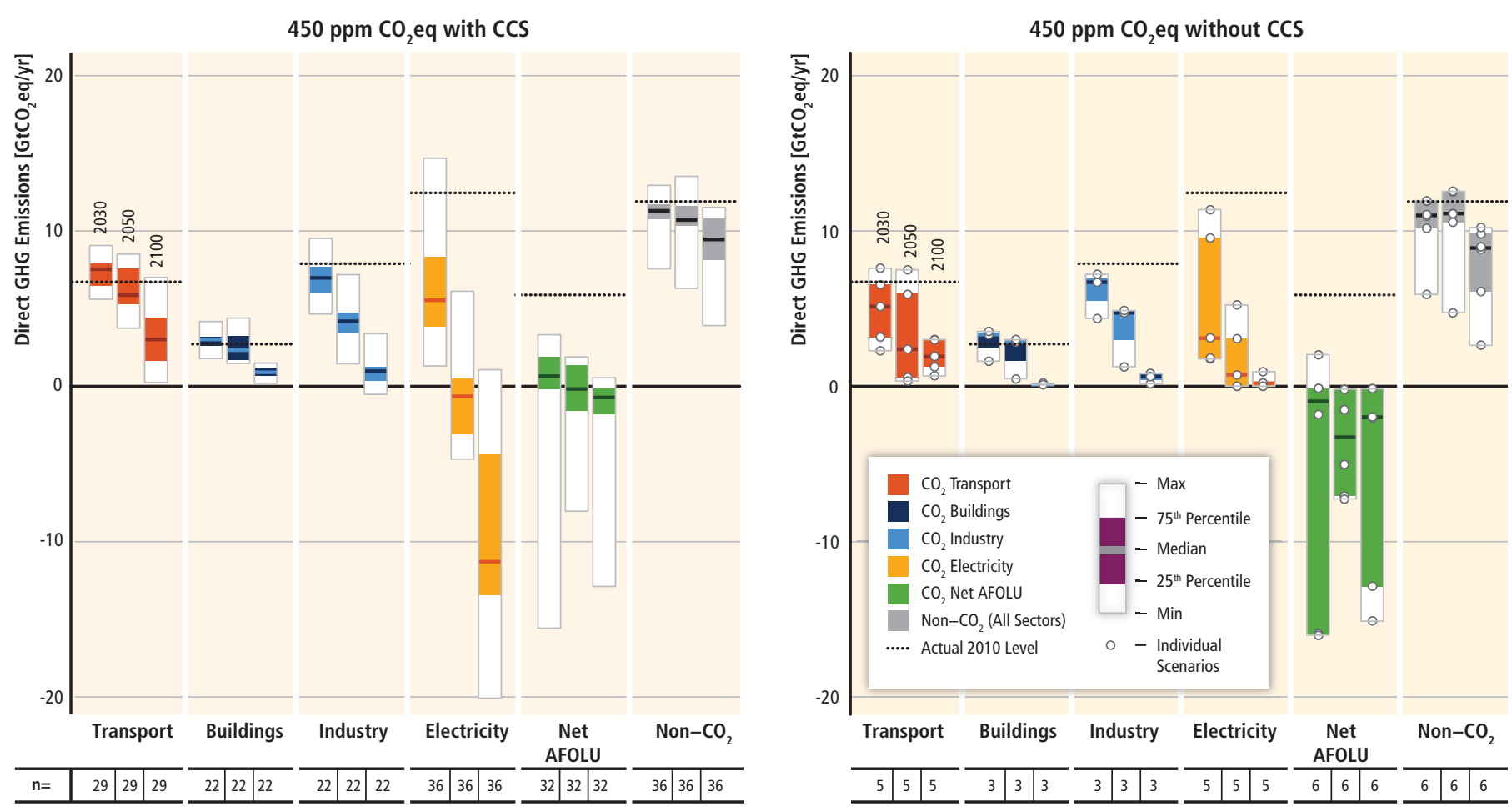

Figure TS.17| Direct emissions of $\mathrm{CO}_{2}$ and non- $\mathrm{CO}_{2}$ GHGs across sectors in mitigation scenarios that reach about 450 (430-480) ppm $\mathrm{CO}_{2}$ eq concentrations in 2100 with using carbon dioxide capture and storage (CCS) (left panel) and without using CCS (right panel). The numbers at the bottom of the graphs refer to the number of scenarios included in the ranges that differ across sectors and time due to different sectoral resolutions and time horizons of models. White dots in the right panel refer to emissions of individual scenarios to give a sense of the spread within the ranges shown due to the small number of scenarios. [Figures 6.35]

space and process heating, and potentially for some modes of transport). Deep emissions reductions in transport are generally the last to emerge in integrated modelling studies because of the limited options to switch to low-carbon energy carriers compared to buildings and industry (Figure TS.17). [6.3.4, 6.8, 8.9, 9.8, 10.10, 7.11, Figure 6.17]

The availability of CDR technologies affects the size of the mitigation challenge for the energy end-use sectors (robust evidence, high agreement) $[6.8,7.11]$. There are strong interdependencies in mitigation scenarios between the required pace of decarbonization of energy supply and end-use sectors. The more rapid decarbonization of supply generally provides more flexibility for the end-use sectors. However, barriers to decarbonizing the supply side, resulting for example from a limited availability of CCS to achieve negative emissions when combined with bioenergy, require a more rapid and pervasive decarbonisation of the energy end-use sectors in scenarios achieving low$\mathrm{CO}_{2}$ eq concentration levels (Figure TS.17). The availability of mature large-scale biomass supply for energy, or carbon sequestration technologies in the AFOLU sector also provides flexibility for the development of mitigation technologies in the energy supply and energy enduse sectors [11.3] (limited evidence, medium agreement), though there may be adverse impacts on sustainable development.

Spatial planning can contribute to managing the development of new infrastructure and increasing system-wide efficiencies across sectors (robust evidence, high agreement). Land use, transport choice, housing, and behaviour are strongly interlinked and shaped by infrastructure and urban form. Spatial and land-use planning, such as mixed-zoning, transport-oriented development, increasing density, and co-locating jobs and homes can contribute to mitigation across sectors by (1) reducing emissions from travel demand for both work and leisure, and enabling non-motorized transport, (2) reducing floor space for housing, and hence (3) reducing overall direct and indirect energy use through efficient infrastructure supply. Compact and in-fill development of urban spaces and intelligent densification can save land for agriculture and bioenergy and preserve land carbon stocks. $[8.4,9.10,10.5$, $11.10,12.2,12.3]$

Interdependencies exist between adaptation and mitigation at the sectoral level and there are benefits from considering adaptation and mitigation in concert (medium evidence, high agreement). Particular mitigation actions can affect sectoral climate vulnerability, both by influencing exposure to impacts and by altering the capacity to adapt to them $[8.5,11.5]$. Other interdependencies include climate impacts on mitigation options, such as forest conservation or hydropower production [11.5.5, 7.7], as well as the effects of particular adaptation options, such as heating or cooling of buildings or establishing more diversified cropping systems in agriculture, on GHG emissions and radiative forcing $[11.5 .4,9.5]$. There is a growing evidence base for such interdependencies in each sector, but there are substantial knowledge gaps that prevent the generation of integrated results at the cross-sectoral level. 
Table TS.3 Main sectoral mitigation measures categorized by key mitigation strategies (in bold) and associated sectoral indicators (highlighted in yellow) as discussed in Chapters 7-12.

\begin{tabular}{|c|c|c|c|c|c|c|c|c|}
\hline & $\begin{array}{l}\text { GHG emissions } \\
\text { intensity reduction }\end{array}$ & \multicolumn{2}{|c|}{$\begin{array}{l}\text { Energy intensity reduction by } \\
\text { improving technical efficiency }\end{array}$} & \multicolumn{2}{|c|}{$\begin{array}{l}\text { Production and resource } \\
\text { efficiency improvement }\end{array}$} & \multicolumn{2}{|c|}{$\begin{array}{l}\text { Structural and systems } \\
\text { efficiency improvement }\end{array}$} & Activity indicator change \\
\hline \multirow[b]{2}{*}{ 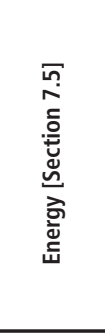 } & $\begin{array}{l}\text { Emissions/ secondary } \\
\text { energy output }\end{array}$ & \multicolumn{2}{|c|}{ Energy input/ energy output } & \multicolumn{2}{|c|}{ Embodied energy/ energy output } & \multicolumn{2}{|c|}{ - } & Final energy use \\
\hline & $\begin{array}{l}\text { Greater deployment of renewable } \\
\text { energy (RE), nuclear energy, } \\
\text { and (BE)CCS; fuel switching } \\
\text { within the group of fossil fuels; } \\
\text { reduction of fugitive (methane) } \\
\text { emissions in the fossil fuel chain }\end{array}$ & \multicolumn{2}{|c|}{$\begin{array}{l}\text { Extraction, transport and } \\
\text { conversion of fossil fuels; } \\
\text { electricity/ heat fuel transmission, } \\
\text { distribution, and storage; } \\
\text { Combined Heat and Power (CHP) } \\
\text { or cogeneration (see Buildings } \\
\text { and Human Settlements) } \\
\end{array}$} & \multicolumn{2}{|c|}{$\begin{array}{l}\text { Energy embodied in manufacturing } \\
\text { of energy extraction, } \\
\text { conversion, transmission and } \\
\text { distribution technologies }\end{array}$} & \multicolumn{2}{|c|}{ Addressing integration needs } & $\begin{array}{l}\text { Demand from end-use sectors } \\
\text { for different energy carriers (see } \\
\text { Transport, Buildings and Industry) }\end{array}$ \\
\hline \multirow[b]{2}{*}{ 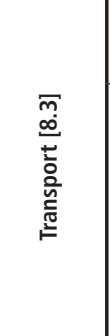 } & Emissions/ final energy & \multicolumn{2}{|c|}{ Final energy/ transport service } & \multicolumn{2}{|c|}{ - } & \multicolumn{2}{|c|}{ Shares for each mode } & Total distance per year \\
\hline & $\begin{array}{l}\text { Fuel carbon intensity } \\
\left(\mathrm{CO}_{2} \text { eq/megajoule (MJ)): }\right. \\
\text { Fuel switching to low-carbon } \\
\text { fuels e. g., electricity/hydrogen } \\
\text { from low-carbon sources (see } \\
\text { Energy); specific biofuels in } \\
\text { various modes (see AFOLU) }\end{array}$ & \multicolumn{2}{|c|}{$\begin{array}{l}\text { Energy intensity } \\
\text { (MJ/passenger-km, tonne- } \\
\text { km): Fuel-efficient engines and } \\
\text { vehicle designs; more advanced } \\
\text { propulsion systems and designs; } \\
\text { use of lighter materials in vehicles }\end{array}$} & \multicolumn{2}{|c|}{$\begin{array}{l}\text { Embodied emissions during } \\
\text { vehicle manufacture; material } \\
\text { efficiency; and recycling of } \\
\text { materials (see Industry); } \\
\text { infrastructure lifecycle emissions } \\
\text { (see Human Settlements) }\end{array}$} & \multicolumn{2}{|c|}{$\begin{array}{l}\text { Modal shifts from light-duty } \\
\text { vehicles (LDVs) to public transit, } \\
\text { cycling/walking, and from aviation } \\
\text { and heavy-duty vehicles (HDVs) } \\
\text { to rail; eco-driving; improved } \\
\text { freight logistics; transport } \\
\text { (infrastructure) planning }\end{array}$} & $\begin{array}{l}\text { Journey avoidance; higher } \\
\text { occupancy/loading rates; reduced } \\
\text { transport demand; urban planning } \\
\text { (see Human Settlements) }\end{array}$ \\
\hline \multirow[b]{2}{*}{ 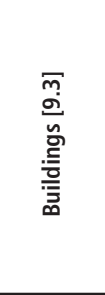 } & Emissions/ final energy & \multicolumn{2}{|c|}{ Final energy/ useful energy } & \multicolumn{2}{|c|}{$\begin{array}{l}\text { Embodied energy/ } \\
\text { operating energy }\end{array}$} & \multicolumn{2}{|c|}{ Useful energy/ energy service } & Energy service demand \\
\hline & $\begin{array}{l}\text { Fuel carbon intensity } \\
\left(\mathrm{CO}_{2} \text { eq/MJ): Building- }\right. \\
\text { integrated RE technologies; fuel } \\
\text { switching to low-carbon fuels, } \\
\text { e. g., electricity (see Energy) }\end{array}$ & \multicolumn{2}{|c|}{$\begin{array}{l}\text { Device efficiency: heating/ } \\
\text { cooling (high-performance boilers, } \\
\text { ventilation, air-conditioning, } \\
\text { heat pumps); water heating; } \\
\text { cooking (advanced biomass } \\
\text { stoves); lighting; appliances }\end{array}$} & \multicolumn{2}{|c|}{$\begin{array}{l}\text { Building lifetime; component, } \\
\text { equipment, and appliance } \\
\text { durability; low(er) energy and } \\
\text { emission material choice for } \\
\text { construction (see Industry) }\end{array}$} & \multicolumn{2}{|c|}{$\begin{array}{l}\text { Systemic efficiency: integrated } \\
\text { design process; low/zero energy } \\
\text { buildings; building automation } \\
\text { and controls; urban planning; } \\
\text { district heating/cooling and CHP; } \\
\text { smart meters/grids; commissioning }\end{array}$} & $\begin{array}{l}\text { Behavioural change (e.g., } \\
\text { thermostat setting, appliance use); } \\
\text { lifestyle change (e.g., per capita } \\
\text { dwelling size, adaptive comfort) }\end{array}$ \\
\hline & Emissions/ final energy & \multicolumn{2}{|c|}{ Final energy/ material production } & \multicolumn{2}{|c|}{ Material input/ product output } & \multicolumn{2}{|c|}{ Product demand/ service demand } & Service demand \\
\hline 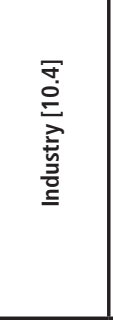 & $\begin{array}{l}\text { Emissions intensity: Process } \\
\text { emissions reductions; use of } \\
\text { waste (e. g., municipal solid waste } \\
\text { (MSW)/sewage sludge in cement } \\
\text { kilns) and CCS in industry; HFCs } \\
\text { replacement and leak repair; } \\
\text { fuel switching among fossil fuels } \\
\text { to low-carbon electricity (see } \\
\text { Energy) or biomass (see AFOLU) }\end{array}$ & \multicolumn{2}{|c|}{$\begin{array}{l}\text { Energy efficiency/ best } \\
\text { available technologies: } \\
\text { Efficient steam systems; } \\
\text { furnace and boiler systems; } \\
\text { electric motor (pumps, fans, } \\
\text { air compressor, refrigerators, } \\
\text { and material handling) and } \\
\text { electronic control systems; (waste) } \\
\text { heat exchanges; recycling }\end{array}$} & \multicolumn{2}{|c|}{$\begin{array}{l}\text { Material efficiency: } \\
\text { Reducing yield losses; } \\
\text { manufacturing/construction: } \\
\text { process innovations, new design } \\
\text { approaches, re-using old material } \\
\text { (e. g., structural steel); product } \\
\text { design (e. g., light weight car } \\
\text { design); fly ash substituting clinker }\end{array}$} & \multicolumn{2}{|c|}{$\begin{array}{l}\text { Product-service efficiency: } \\
\text { More intensive use of products } \\
\text { (e.g., car sharing, using products } \\
\text { such as clothing for longer, new } \\
\text { and more durable products) }\end{array}$} & $\begin{array}{l}\text { Reduced demand for, e.g., } \\
\text { products such as clothing; } \\
\text { alternative forms of travel } \\
\text { leading to reduced demand } \\
\text { for car manufacturing }\end{array}$ \\
\hline \multirow{2}{*}{ 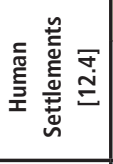 } & Emissions/ final energy & \multicolumn{2}{|c|}{ Final energy/ useful energy } & \multicolumn{2}{|c|}{ Material input in infrastructure } & \multicolumn{2}{|c|}{ Useful energy/ energy service } & Service demand per capita \\
\hline & $\begin{array}{l}\text { Integration of urban } \\
\text { renewables; urban-scale fuel } \\
\text { switching programmes }\end{array}$ & \multicolumn{2}{|c|}{$\begin{array}{l}\text { Cogeneration, heat cascading, } \\
\text { waste to energy }\end{array}$} & \multicolumn{2}{|c|}{$\begin{array}{l}\text { Managed infrastructure supply; } \\
\text { reduced primary material } \\
\text { input for infrastructure }\end{array}$} & \multicolumn{2}{|c|}{$\begin{array}{l}\text { Compact urban form; increased } \\
\text { accessibility; mixed land use }\end{array}$} & $\begin{array}{l}\text { Increasing accessibility: } \\
\text { shorter travel time, and more } \\
\text { transport mode options }\end{array}$ \\
\hline \multirow[b]{3}{*}{ 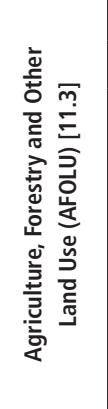 } & & & upply-side improve & nents & & & & mand-side measures \\
\hline & Emissions/area or unit product (co & served, 1 & & & & & Animal/crop proc & uct consumption per capita \\
\hline & $\begin{array}{l}\text { Emissions reduction: of methan } \\
\text { livestock management) and nitrous } \\
\text { (fertilizer and manure managemen } \\
\text { and prevention of emissions to the } \\
\text { atmosphere by conserving existing } \\
\text { pools in soils or vegetation (reduci } \\
\text { deforestation and forest degradati } \\
\text { prevention/control, agroforestry); } \\
\text { emissions intensity (GHG/unit proc }\end{array}$ & & $\begin{array}{l}\text { Sequestration: Inc } \\
\text { size of existing carbo } \\
\text { thereby extracting CC } \\
\text { atmosphere (e.g., af } \\
\text { reforestation, integra } \\
\text { carbon sequestration }\end{array}$ & $\begin{array}{l}\text { asing the } \\
\text { pools, } \\
\text { from the } \\
\text { restation, } \\
\text { ed systems, } \\
\text { in soils) }\end{array}$ & $\begin{array}{l}\text { Substitution: of } \\
\text { products for fossi } \\
\text { energy-intensive } \\
\text { thereby reducing } \\
\text { e.g., biomass co- } \\
\text { Energy), biofuels } \\
\text { biomass-based st } \\
\text { insulation produc }\end{array}$ & $\begin{array}{l}\text { f biological } \\
\text { I fuels or } \\
\text { products, } \\
\mathrm{CO}_{2} \text { emissions, } \\
\text { firing/CHP (see } \\
\text { (see Transport), } \\
\text { oves, and } \\
\text { ts (see Buildings) }\end{array}$ & $\begin{array}{l}\text { Demand-side } \mathbf{n} \\
\text { and wastes of foc } \\
\text { towards less emis } \\
\text { use of long-lived }\end{array}$ & $\begin{array}{l}\text { leasures: Reducing losses } \\
\text { d; changes in human diets } \\
\text { sion-intensive products; } \\
\text { wood products }\end{array}$ \\
\hline
\end{tabular}




\section{TS.3.2.2 Energy supply}

The energy supply sector is the largest contributor to global GHG emissions (robust evidence, high agreement). Annual GHG emissions from the global energy supply sector grew more rapidly between 2000 and 2010 than in the previous decade; their growth accelerated from 1.7\%/yr from 1990-2000 to 3.1\%/yr from 2000-2010. The main contributors to this trend are an increasing demand for energy services and a growing share of coal in the global fuel mix. The energy supply sector, as defined in this report, comprises all energy extraction, conversion, storage, transmission, and distribution processes that deliver final energy to the end-use sectors (industry, transport, buildings, agriculture and forestry). [7.2, 7.3]

In the baseline scenarios assessed in AR5, direct $\mathrm{CO}_{2}$ emissions from the energy supply sector increase from $14.4 \mathrm{GtCO}_{2} / \mathrm{yr}$ in 2010 to $24-33 \mathrm{GtCO}_{2} / \mathrm{yr}$ in 2050 (25-75th percentile; full range $15-42 \mathrm{GtCO}_{2} / \mathrm{yr}$ ), with most of the baseline scenarios assessed in WGIII AR5 showing a significant increase (medium evidence, medium agreement) (Figure TS.15). The lower end of the full range is dominated by scenarios with a focus on energy intensity improvements that go well beyond the observed improvements over the past 40 years. The availability of fossil fuels alone will not be sufficient to limit $\mathrm{CO}_{2}$ eq concentration to levels such as $450 \mathrm{ppm}$, $550 \mathrm{ppm}$, or $650 \mathrm{ppm}$. [6.3.4, 6.8, 7.11, Figure 6.15]

The energy supply sector offers a multitude of options to reduce GHG emissions (robust evidence, high agreement). These options include: energy efficiency improvements and fugitive emission reductions in fuel extraction as well as in energy conversion, transmission, and distribution systems; fossil fuel switching; and low-GHG energy supply technologies such as renewable energy (RE), nuclear power, and CCS (Table TS.3). [7.5, 7.8.1, 7.11]

The stabilization of GHG concentrations at low levels requires a fundamental transformation of the energy supply system, including the long-term phase-out of unabated fossil fuel conversion technologies and their substitution by low-GHG alternatives (robust evidence, high agreement). Concentrations of $\mathrm{CO}_{2}$ in the atmosphere can only be stabilized if global (net) $\mathrm{CO}_{2}$ emissions peak and decline toward zero in the long term. Improving the energy efficiencies of fossil fuel power plants and/or the shift from coal to gas will not by themselves be sufficient to achieve this. Low-GHG energy supply technologies would be necessary if this goal were to be achieved (Figure TS.19). [7.5.1, 7.8.1, 7.11]

Decarbonizing (i.e., reducing the carbon intensity of) electricity generation is a key component of cost-effective mitigation strategies in achieving low-stabilization levels $(430-530 \mathrm{ppm}$ $\mathrm{CO}_{2} \mathrm{eq}$ ); in most integrated modelling scenarios, decarbonization happens more rapidly in electricity generation than in the buildings, transport, and industry sectors (medium evidence, high agreement) (Figure TS.17). In the majority of mitigation scenar- ios reaching about $450 \mathrm{ppm} \mathrm{CO}_{2}$ eq concentrations by 2100 , the share of low-carbon electricity supply (comprising RE, nuclear, fossil fuels with (CS, and BECCS) increases from the current share of around $30 \%$ to more than $80 \%$ by 2050 , and fossil fuel power generation without CCS is phased out almost entirely by 2100 (Figures TS.17 and TS.18) [7.14].

Since AR4, many RE technologies have demonstrated substantial performance improvements and cost reductions, and a growing number of RE technologies have achieved a level of maturity to enable deployment at significant scale (robust evidence, high agreement). Some technologies are already economically competitive in various settings. Levelized costs of PV systems fell most substantially between 2009 and 2012, and a less extreme trend has been observed for many others RE technologies. Regarding electricity generation alone, RE accounted for just over half of the new electricity-generating capacity added globally in 2012, led by growth in wind, hydro, and solar power. Decentralized RE to meet rural energy needs has also increased, including various modern and advanced traditional biomass options as well as small hydropower, PV, and wind. Nevertheless, many RE technologies still need direct support (e. g., feed-in tariffs (FITs), RE quota obligations, and tendering/bidding) and/or indirect support (e.g., sufficiently high carbon prices and the internalization of other externalities), if their market shares are to be significantly increased. RE technology policies have been successful in driving the recent growth of RE. Additional enabling policies are needed to address their integration into future energy systems. (medium evidence, medium agreement) (Figure TS.19) [7.5.3, $7.6 .1,7.8 .2,7.12,11.13]$

The use of RE is often associated with co-benefits, including the reduction of air pollution, local employment opportunities, few severe accidents compared to some other energy supply technologies, as well as improved energy access and security (medium evidence, medium agreement) (Table TS.4). At the same time, however, some RE technologies can have technology and location-specific adverse side-effects, which can be reduced to a degree through appropriate technology selection, operational adjustments, and siting of facilities. [7.9]

Infrastructure and integration challenges vary by RE technology and the characteristics of the existing energy system (medium evidence, medium agreement). Operating experience and studies of medium to high penetrations of RE indicate that integration issues can be managed with various technical and institutional tools. As RE penetrations increase, such issues are more challenging, must be carefully considered in energy supply planning and operations to ensure reliable energy supply, and may result in higher costs. [7.6, 7.8.2]

Nuclear energy is a mature low-GHG emission source of baseload power, but its share of global electricity generation has been declining (since 1993). Nuclear energy could make an increasing contribution to low-carbon energy supply, but a variety of barriers and risks exist (robust evidence, high agree- 

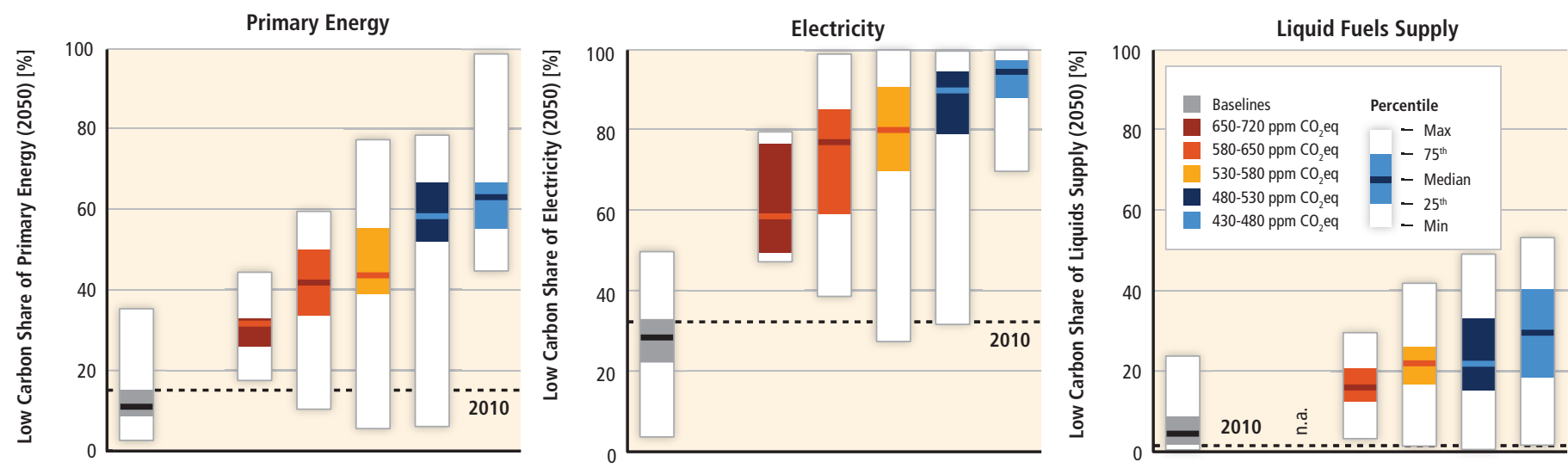

Figure TS.18 Share of low-carbon energy in total primary energy, electricity and liquid fuels supply sectors for the year 2050. Dashed horizontal lines show the low-carbon share for the year 2010. Low-carbon energy includes nuclear, renewables, fossil fuels with carbon dioxide capture and storage (CCS) and bioenergy with CCS. [Figure 7.14]

ment) (Figure TS.19). Nuclear electricity accounted for $11 \%$ of the world's electricity generation in 2012, down from a high of $17 \%$ in 1993. Pricing the externalities of GHG emissions (carbon pricing) could improve the competitiveness of nuclear power plants. [7.2, 7.5.4, 7.8.1, 7.12]

Barriers and risks associated with an increasing use of nuclear energy include operational risks and the associated safety concerns, uranium mining risks, financial and regulatory risks, unresolved waste management issues, nuclear weapon proliferation concerns, and adverse public opinion (robust evidence, high agreement) (Table TS.4). New fuel cycles and reactor technologies addressing some of these issues are under development and progress has been made concerning safety and waste disposal. Investigation of mitigation scenarios not exceeding $580 \mathrm{ppm} \mathrm{CO}_{2}$ eq has shown that excluding nuclear power from the available portfolio of technologies would result in only a slight increase in mitigation costs compared to the full technology portfolio (Figure TS.13). If other technologies, such as CCS, are constrained the role of nuclear power expands. [6.3.6, 7.5.4, 7.8.2, 7.9, 7.11]

GHG emissions from energy supply can be reduced significantly by replacing current world average coal-fired power plants with modern, highly efficient natural gas combined cycle power plants or combined heat and power (CHP) plants, provided that natural gas is available and the fugitive emissions associated with its extraction and supply are low or mitigated (robust evidence, high agreement). In mitigation scenarios reaching about $450 \mathrm{ppm} \mathrm{CO}_{2}$ eq concentrations by 2100 , natural gas power generation without CCS typically acts as a bridge technology, with deployment increasing before peaking and falling to below current levels by 2050 and declining further in the second half of the century (robust evidence, high agreement). [7.5.1, 7.8, 7.9, 7.11, 7.12]

Carbon dioxide capture and storage (CCS) technologies could reduce the lifecycle GHG emissions of fossil fuel power plants (medium evidence, medium agreement). While all components of integrated CCS systems exist and are in use today by the fossil fuel extraction and refining industry, CCS has not yet been applied at scale to a large, commercial fossil fuel power plant. CCS power plants could be seen in the market if they are required for fossil fuel facilities by regulation or if they become competitive with their unabated counterparts, for instance, if the additional investment and operational costs faced by CCS plants, caused in part by efficiency reductions, are compensated by sufficiently high carbon prices (or direct financial support). Beyond economic incentives, well-defined regulations concerning short- and long-term responsibilities for storage are essential for a large-scale future deployment of CCS. [7.5.5]

Barriers to large-scale deployment of CCS technologies include concerns about the operational safety and long-term integrity of $\mathrm{CO}_{2}$ storage, as well as risks related to transport and the required up-scaling of infrastructure (limited evidence, medium agreement) (Table TS.4). There is, however, a growing body of literature on how to ensure the integrity of $\mathrm{CO}_{2}$ wells, on the potential consequences of a $\mathrm{CO}_{2}$ pressure build-up within a geologic formation (such as induced seismicity), and on the potential human health and environmental impacts from $\mathrm{CO}_{2}$ that migrates out of the primary injection zone (limited evidence, medium agreement). [7.5.5, 7.9, 7.11]

Combining bioenergy with CCS (BECCS) offers the prospect of energy supply with large-scale net negative emissions, which plays an important role in many low-stabilization scenarios, while it entails challenges and risks (limited evidence, medium agreement). Until 2050, bottom-up studies estimate the economic potential to be between $2-10 \mathrm{GtCO}_{2}$ per year [11.13]. Some mitigation scenarios show higher deployment of BECCS towards the end of the century. Technological challenges and risks include those associated with the upstream provision of the biomass that is used in the CCS facility, as well as those associated with the CCS technology itself. Currently, no large-scale projects have been financed. [6.9, 7.5.5, 7.9, 11.13] 
Scenarios Reaching 430-530 ppm CO 2 eq in 2100 in Integrated Models Emission Intensity of Electricity $\left[\mathrm{gCO}_{2} / \mathrm{kWh}\right]$

\begin{tabular}{c|c|c|c|c|c|}
$1000 \quad 800$ & 400 & 200 & & \\
\hline $\begin{array}{l}\text { Direct Emission Intensity } \\
\text { Global Average, 2030 }\end{array}$ & & & & & \\
Global Average, 2050 & & & & & \\
\hline
\end{tabular}

$-200$

\section{(1)}

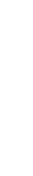

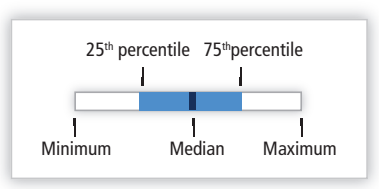

\section{Currently Commercially Available Technologies}

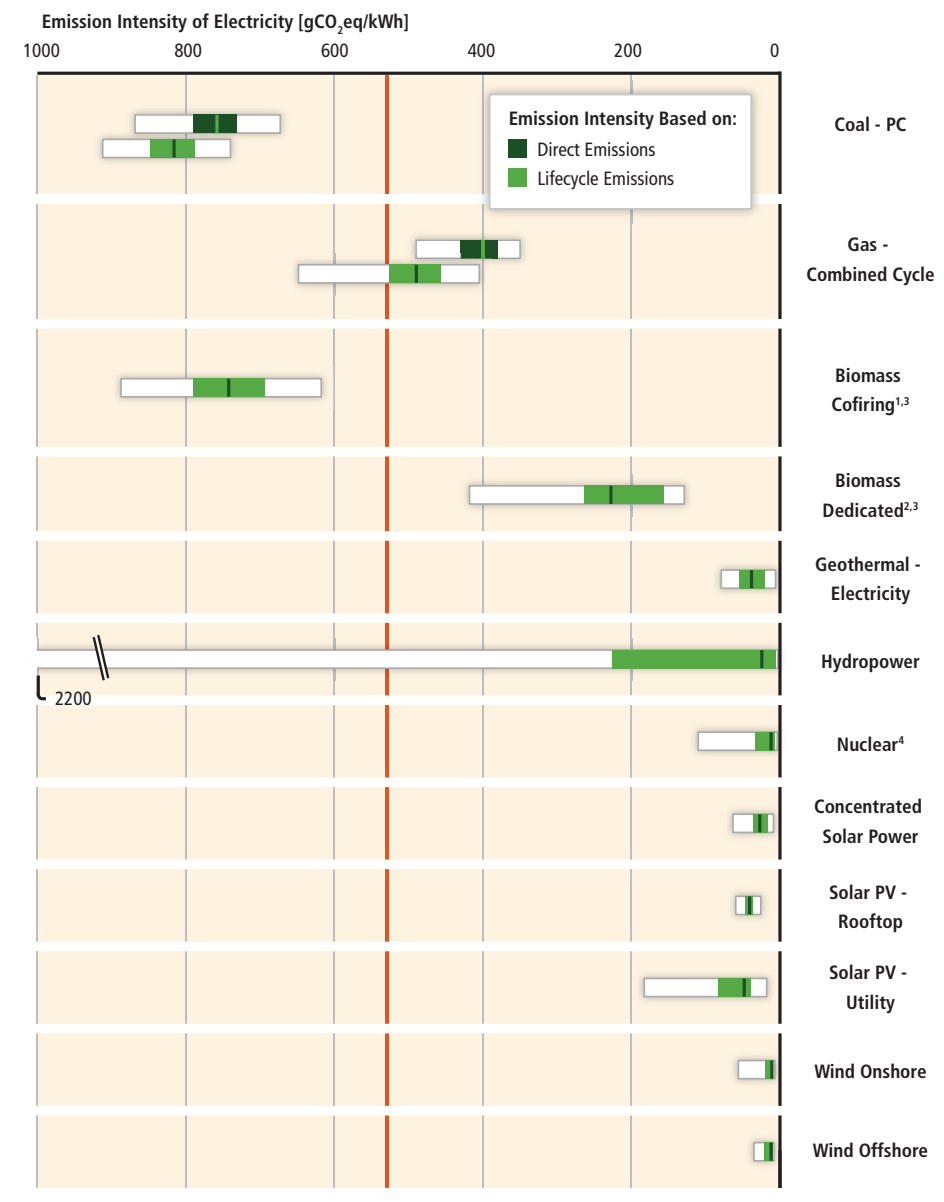

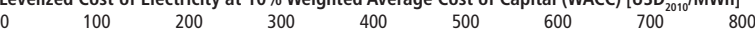

\section{Pre-commercial Technologies}
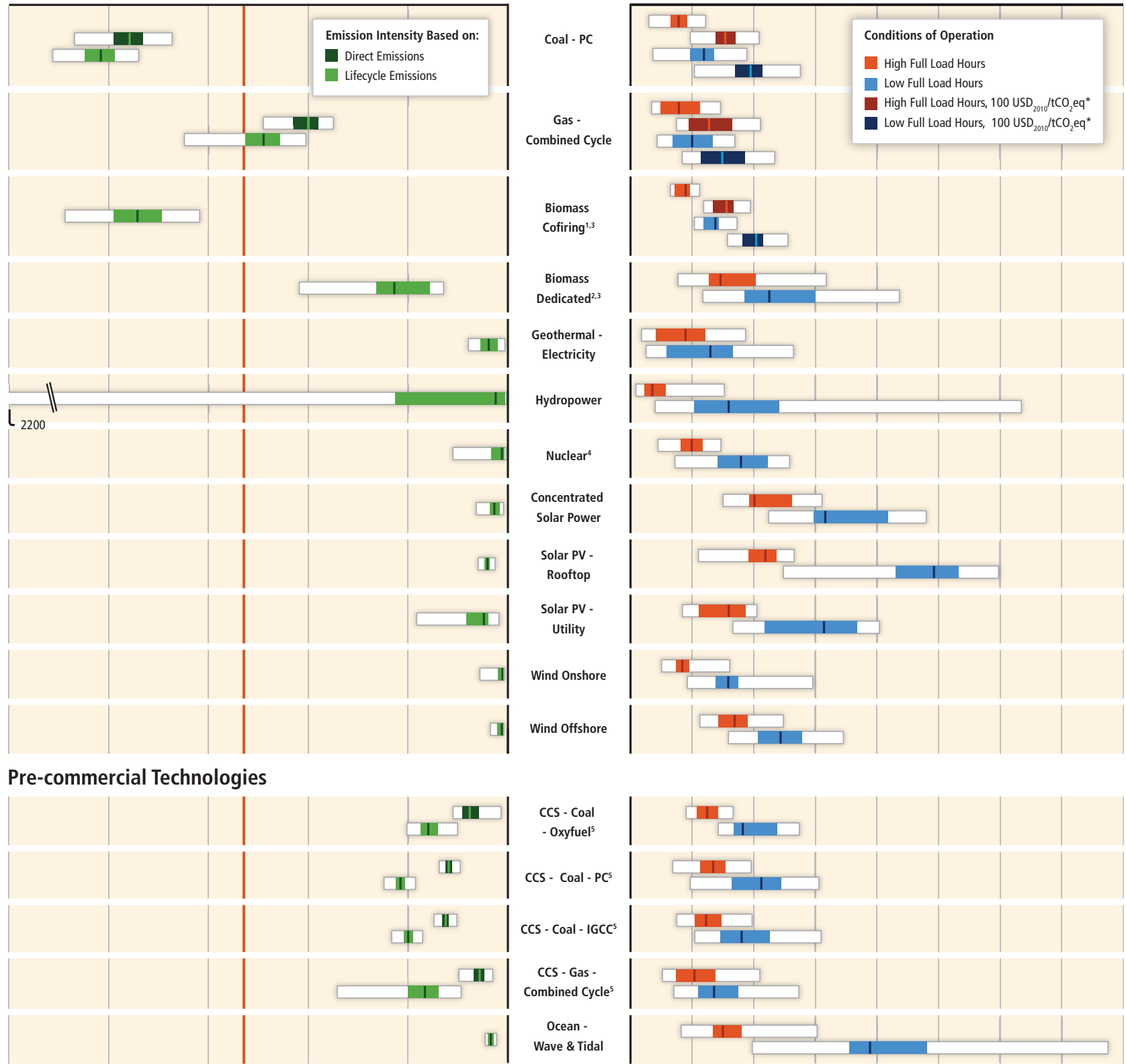

'Assuming biomass feedstocks are dedicated energy plants and crop residues and $80-95 \%$ coal input. ${ }^{2}$ Assuming feedstocks are dedicated energy plants and crop residues. Direct emissions of biomass power plants are not shown explicitly, but included in the lifecycle emissions. Lifecycle emissions include albedo effect.

${ }^{4} \mathrm{LCOE}$ of nuclear include front and back-end fuel costs as well as decommissioning costs.

${ }^{5}$ Transport and storage costs of $\mathrm{CCS}$ are set to $10 \mathrm{USD}_{2010} / \mathrm{tCO}$

* Carbon price levied on direct emissions. Effects shown where significant.

Figure TS.19| Specific direct and lifecycle emissions ( $\mathrm{gCO}_{2}$ eq/ kilowatt hour $(\mathrm{kWh})$ ) and levelized cost of electricity (LCOE in USD $2010 / \mathrm{MWh}$ ) for various power-generating technologies (see Annex III.2 for data and assumptions and Annex II.3.1 and II.9.3 for methodological issues). The upper left graph shows global averages of specific direct $\mathrm{CO}_{2}$ emissions $\left(\mathrm{gCO}_{2} / \mathrm{kWh}\right)$ of power generation in 2030 and 2050 for the set of about 450 to about 500 (430-530) ppm $\mathrm{CO}_{2}$ eq scenarios that are contained in the WG III AR5 Scenario Database (see Annex II. 10). The global average of specific direct $\mathrm{CO}_{2}$ emissions $\left(\mathrm{gCO}_{2} / \mathrm{kWh}\right)$ of power generation in 2010 is shown as a vertical line. Note: The inter-comparability of $\mathrm{LCOE}$ is limited. For details on general methodological issues and interpretation see Annexes as mentioned above. CCS: $\mathrm{CO}_{2}$ capture and storage; IGCC: Integrated coal gasification combined cycle; PC: Pulverized hard coal; PV: Photovoltaic; WACC: Weighted average cost of capital. [Figure 7.7] 
Table TS.4| Overview of potential co-benefits (green arrows) and adverse side-effects (orange arrows) of the main mitigation measures in the energy supply sector; arrows pointing up/down denote a positive/negative effect on the respective objective or concern; a question mark (?) denotes an uncertain net effect. Co-benefits and adverse side-effects depend on local circumstances as well as on the implementation practice, pace, and scale. For possible upstream effects of biomass supply for bioenergy, see Table TS.8. For an assessment of macroeconomic, cross-sectoral effects associated with mitigation policies (e. g., on energy prices, consumption, growth, and trade), see e. g., Sections 3.9, 6.3.6, 13.2.2.3 and 14.4.2. The uncertainty qualifiers in brackets denote the level of evidence and agreement on the respective effects (see TS.1). Abbreviations for evidence: $I=$ limited, $m=$ medium, r=robust; for agreement: I=low, m=medium, h=high. [Table 7.3]

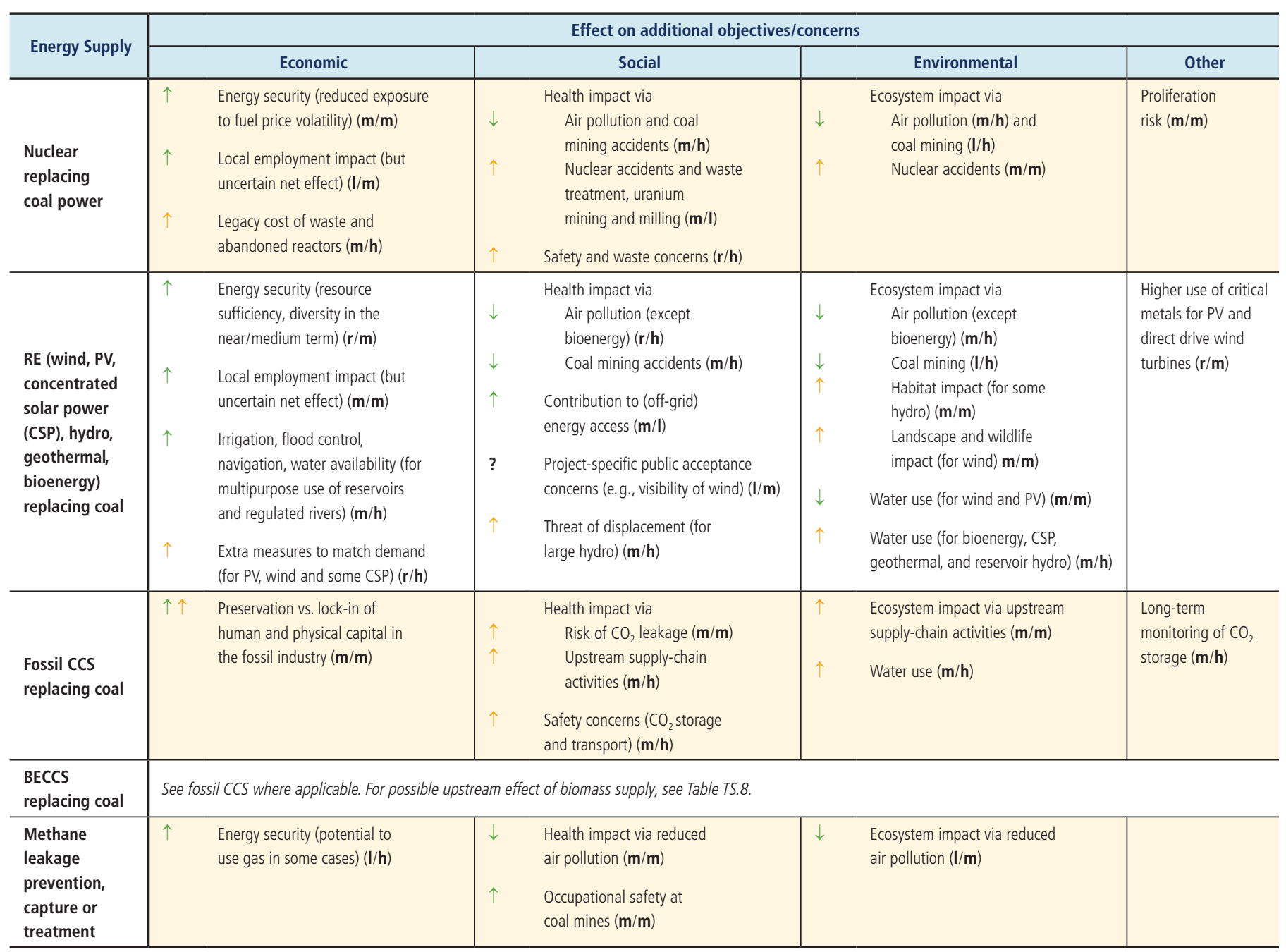

\section{TS.3.2.3 Transport}

Since AR4, emissions in the global transport sector have grown in spite of more efficient vehicles (road, rail, watercraft, and aircraft) and policies being adopted (robust evidence, high agreement). Road transport dominates overall emissions but aviation could play an increasingly important role in total $\mathrm{CO}_{2}$ emissions in the future. $[8.1,8.3,8.4]$

The global transport sector accounted for $27 \%$ of final energy use and $6.7 \mathrm{GtCO}_{2}$ direct emissions in 2010, with baseline $\mathrm{CO}_{2}$ emissions projected to increase to $9.3-12 \mathrm{GtCO}_{2} / \mathrm{yr}$ in 2050 (25-75th percentile; full range $6.2-16 \mathrm{GtCO}_{2} / \mathrm{yr}$ ); most of the baseline scenarios assessed in WGIII AR5 foresee a significant increase (medium evidence/medium agreement) (Figure TS.15). With- out aggressive and sustained mitigation policies being implemented, transport sector emissions could increase faster than in the other energy end-use sectors and could lead to more than a doubling of $\mathrm{CO}_{2}$ emissions by 2050. $[6.8,8.9,8.10]$

While the continuing growth in passenger and freight activity constitutes a challenge for future emission reductions, analyses of both sectoral and integrated studies suggest a higher mitigation potential in the transport sector than reported in the AR4 (medium evidence, medium agreement). Transport energy demand per capita in developing and emerging economies is far lower than in OECD countries but is expected to increase at a much faster rate in the next decades due to rising incomes and the development of infrastructure. Baseline scenarios thus show increases in transport energy demand from 2010 out to 2050 and beyond. However, sectoral and 
integrated mitigation scenarios indicate that energy demand reductions of $10-45 \%$ are possible by 2050 relative to baseline (Figure TS.20, left panel) (medium evidence, medium agreement). [6.8.4, 8.9.1, 8.9.4, 8.12, Figure 8.9.4]

A combination of low-carbon fuels, the uptake of improved vehicle and engine performance technologies, behavioural change leading to avoided journeys and modal shifts, investments in related infrastructure and changes in the built environment, together offer a high mitigation potential (high confidence) $[8.3,8.8]$. Direct (tank-to-wheel) GHG emissions from passenger and freight transport can be reduced by:

- using fuels with lower carbon intensities $\left(\mathrm{CO}_{2}\right.$ eq/ megajoule (MJ));

- lowering vehicle energy intensities

(MJ/passenger-km or MJ/tonne-km);

- encouraging modal shift to lower-carbon passenger and freight transport systems coupled with investment in infrastructure and compact urban form; and

- avoiding journeys where possible (Table TS.3).

Other short-term mitigation strategies include reducing black carbon (BC), aviation contrails, and nitrogen oxides $\left(\mathrm{NO}_{\mathrm{x}}\right)$ emissions. [8.4]

Strategies to reduce the carbon intensities of fuel and the rate of reducing carbon intensity are constrained by challenges associated with energy storage and the relatively low energy density of low-carbon transport fuels; integrated and sectoral studies broadly agree that opportunities for fuel switching exist in the short term and will grow over time (medium evidence, medium agreement) (Figure TS.20, right panel). Electric, hydrogen, and some biofuel technologies could help reduce the carbon intensity of fuels, but their total mitigation potentials are very uncertain (medium evidence, medium agreement). Methane-based fuels are already increasing their share for road vehicles and waterborne craft. Electricity produced from low-carbon sources has near-term potential for electric rail and short- to medium-term potential as electric buses, light-duty and 2-wheel road vehicles are deployed. Hydrogen fuels from low-carbon sources constitute longer-term options. Commercially available liquid and gaseous biofuels already provide co-benefits together with mitigation options that can be increased by technology advances, particularly drop-in biofuels for aircraft. Reducing transport emissions of particulate matter (including BC), tropospheric ozone and aerosol precursors (including $\mathrm{NO}_{x}$ ) can have human health and mitigation co-benefits in the short term (medium evidence, medium agreement). Up to 2030, the majority of integrated studies expect a continued reliance on liquid and gaseous fuels, supported by an increase in the use of biofuels. During the second half of the century, many integrated studies also show substantial shares of electricity and/or hydrogen to fuel electric and fuel-cell light-duty vehicles (LDVs). [8.2, 8.3, 11.13]

Energy efficiency measures through improved vehicle and engine designs have the largest potential for emissions reduc-
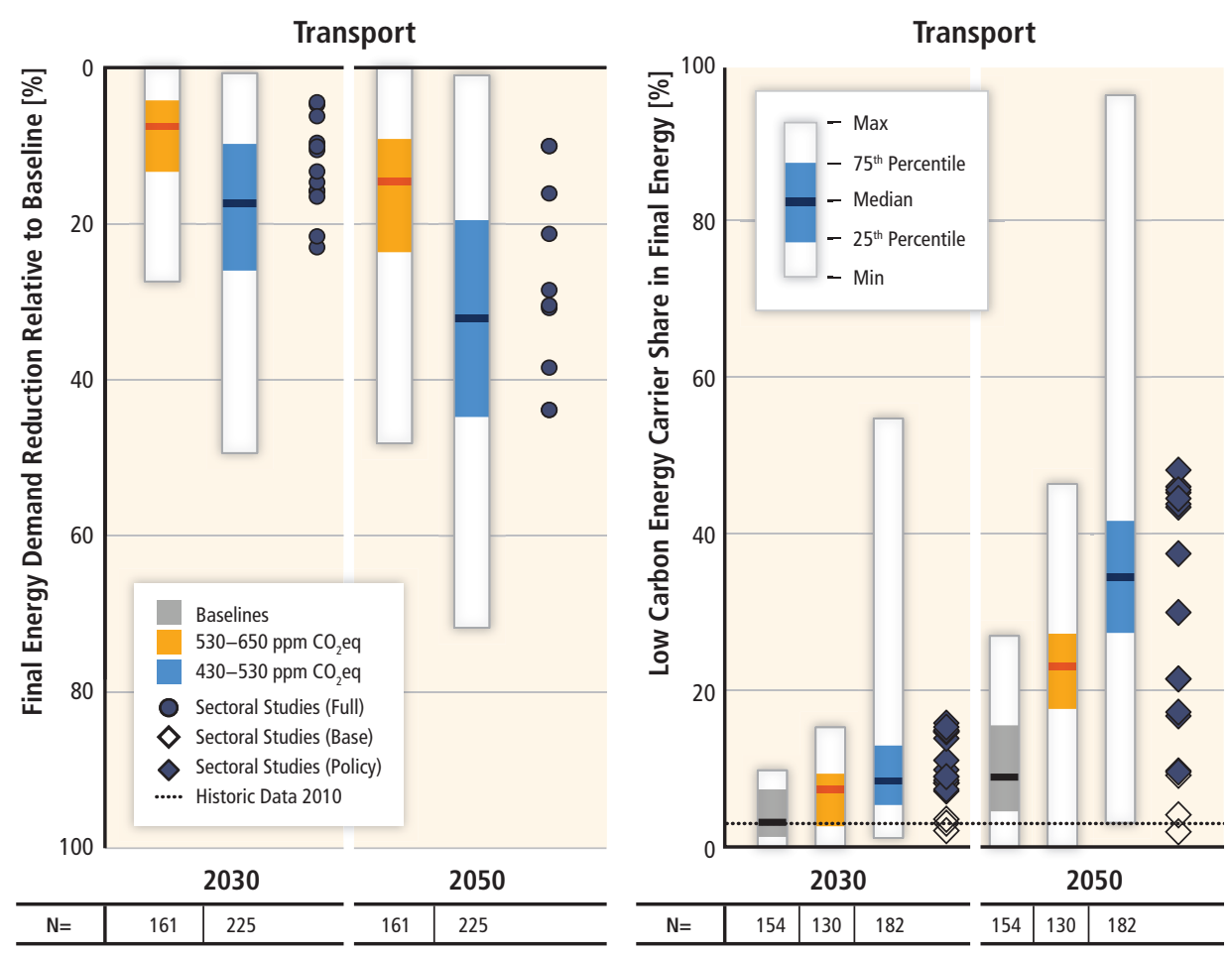

Figure TS.20 Final energy demand reduction relative to baseline (left panel) and development of final low-carbon energy carrier share in final energy (including electricity, hydrogen, and liquid biofuels; right panel) in transport by 2030 and 2050 in mitigation scenarios from three different $\mathrm{CO}_{2}$ eq concentrations ranges shown in boxplots (see Section 6.3.2) compared to sectoral studies shown in shapes assessed in Chapter 8. Filled circles correspond to sectoral studies with full sectoral coverage. [Figures 6.37 and 6.38] 
tions in the short term (high confidence). Potential energy efficiency and vehicle performance improvements range from $30-50 \%$ relative to 2010 depending on transport mode and vehicle type (Figures TS.21, TS.22). Realizing this efficiency potential will depend on large investments by vehicle manufacturers, which may require strong incentives and regulatory policies in order to achieve GHG emissions reduction goals (medium evidence, medium agreement). [8.3, 8.6, 8.9, 8.10]

Shifts in transport mode and behaviour, impacted by new infrastructure and urban (re)development, can contribute to the reduction of transport emissions (medium evidence, low agreement). Over the medium term (up to 2030) to long term (to 2050 and beyond), urban redevelopment and investments in new infrastructure, linked with integrated urban planning, transit-oriented development, and more compact urban form that supports cycling and walking can all lead to modal shifts. Such mitigation measures are challenging, have uncertain outcomes, and could reduce transport GHG emissions by 20-50\% compared to baseline (limited evidence, low agreement). Pricing strategies, when supported by public acceptance initiatives and public and non-motorized transport infrastructures, can reduce travel demand, increase the demand for more efficient vehicles (e.g., where fuel economy standards exist) and induce a shift to low-carbon modes (medium evidence, medium agreement). While infrastructure investments may appear expensive at the margin, the case for sustainable urban planning and related policies is reinforced when co-benefits, such as improved health, accessibility, and resilience, are accounted for (Table TS.5). Business initiatives to decarbonize freight transport have begun but will need further support from fiscal, regulatory, and advisory policies to encourage shifting from road to low-carbon modes such as rail or waterborne options where feasible, as well as improving logistics (Figure TS.22). [8.4, 8.5, 8.7, 8.8, 8.9, 8.10]

Sectoral and integrated studies agree that substantial, sustained, and directed policy interventions could limit transport emissions to be consistent with low concentration goals, but the societal mitigation costs (USD/ $\mathrm{tCO}_{2} \mathrm{eq}$ avoided) remain uncertain (Figures TS.21, TS.22, TS.23). There is good potential to reduce emissions from LDVs and long-haul heavy-duty vehicles (HDVs) from both lower energy intensity vehicles and fuel switching, and the levelized costs of conserved carbon (LCCC) for efficiency improvements can be very low and negative (limited evidence, low agreement). Rail, buses, two-wheel motorbikes, and waterborne craft for freight already have relatively low emissions so their emissions reduction potential is limited. The mitigation cost of electric vehicles is currently high, especially if using grid electricity with a high emissions factor, but their LCCC are expected to decline by 2030 . The emissions intensity of aviation could decline by around $50 \%$ in 2030 but the LCCC, although uncertain, are probably over USD $100 / \mathrm{tCO}_{2}$ eq. While it is expected that mitigation costs will decrease in the future, the magnitude of such reductions is uncertain. (limited evidence, low agreement) [8.6, 8.9]
Barriers to decarbonizing transport for all modes differ across regions but can be overcome, in part, through economic incentives (medium evidence, medium agreement). Financial, institutional, cultural, and legal barriers constrain low-carbon technology uptake and behavioural change. They include the high investment costs needed to build low-emissions transport systems, the slow turnover of stock and infrastructure, and the limited impact of a carbon price on petroleum fuels that are already heavily taxed. Regional differences are likely due to cost and policy constraints. Oil price trends, price instruments on GHG emissions, and other measures such as road pricing and airport charges can provide strong economic incentives for consumers to adopt mitigation measures. [8.8]

There are regional differences in transport mitigation pathways with major opportunities to shape transport systems and infrastructure around low-carbon options, particularly in developing and emerging countries where most future urban growth will occur (robust evidence, high agreement). Possible transformation pathways vary with region and country due to differences in the dynamics of motorization, age and type of vehicle fleets, existing infrastructure, and urban development processes. Prioritizing infrastructure for pedestrians, integrating non-motorized and transit services, and managing excessive road speed for both urban and rural travellers can create economic and social co-benefits in all regions. For all economies, especially those with high rates of urban growth, investments in public transport systems and low-carbon infrastructure can avoid lock-in to carbon-intensive modes. Established infrastructure may limit the options for modal shift and lead to a greater reliance on advanced vehicle technologies; a slowing of growth in LDV demand is already evident in some OECD countries. (medium evidence, medium agreement) $[8.4,8.9]$

A range of strong and mutually supportive policies will be needed for the transport sector to decarbonize and for the co-benefits to be exploited (robust evidence, high agreement). Transport mitigation strategies associated with broader non-climate policies at all government levels can usually target several objectives simultaneously to give lower travel costs, improved access and mobility, better health, greater energy security, improved safety, and increased time savings. Activity reduction measures have the largest potential to realize co-benefits. Realizing the co-benefits depends on the regional context in terms of economic, social, and political feasibility as well as having access to appropriate and cost-effective advanced technologies (Table TS.5). (medium evidence, high agreement) Since rebound effects can reduce the $\mathrm{CO}_{2}$ benefits of efficiency improvements and undermine a particular policy, a balanced package of policies, including pricing initiatives, could help to achieve stable price signals, avoid unintended outcomes, and improve access, mobility, productivity, safety, and health (medium evidence, medium agreement). $[8.4,8.7,8.10]$ 


\section{Passenger Transport}

\section{Currently Commercially Available and Future (2030) Expected Technologies}

Emissions Intensity ( $\left.\mathrm{gCO}_{2} \mathrm{eq} / \mathrm{p}-\mathrm{km}\right)$

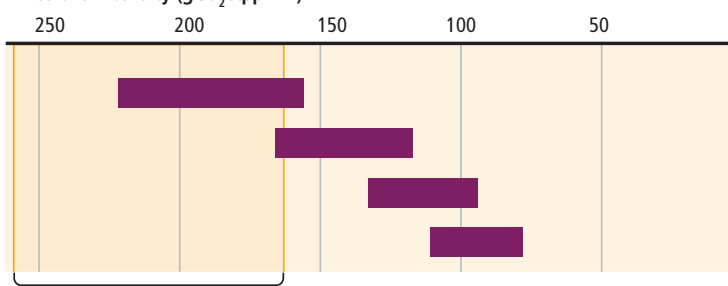

2010 Stock Average SUV

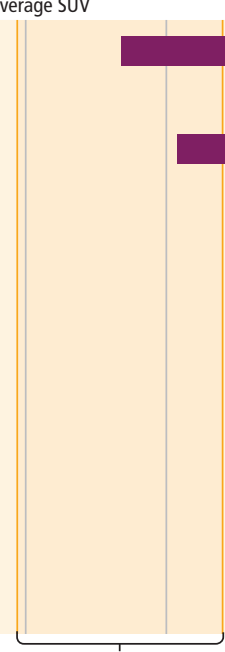

2010 Stock Average LDV
Road

New Sport Utility Vehicles (SUV), Mid-Size

2010 Gasoline

2010 Hybrid Gasoline

2030 Gasoline

2030 Hybrid Gasoline

New Light Duty Vehicles (LDV), Mid-Size

2010 Gasoline

2010 Hybrid Gasoline

2010 Diesel

2010 Compressed Natural Gas

2010 Electric, $600 \mathrm{~g} \mathrm{CO}_{2}$ eq/ $/ \mathrm{kWh}_{\mathrm{el}}$

2010 Electric, $200 \mathrm{~g} \mathrm{CO}_{2}$ eq/kWh

2030 Gasoline

2030 Hybrid Gasoline

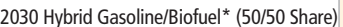

2030 Diesel

2030 Compressed Natural Gas

2030 Electric, $200 \mathrm{~g} \mathrm{CO}_{2}$ eq $/ \mathrm{kWh}_{\mathrm{el}}$

New 2 Wheeler (Scooter Up to $200 \mathrm{~cm}^{3}$ Cylinder Capacity)

2010 Gasoline

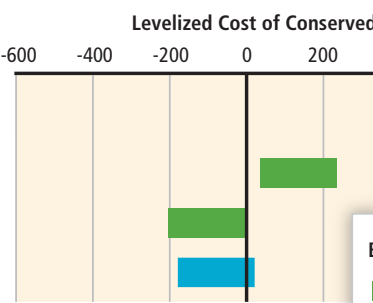

Baselines for LCCC Calculation

New Gasoline SUV (2010)

- New Gasoline LDV (2010)

Optimized Gasoline SUV (2030)

Optimized Gasoline LDV (2030)

Average New Aircraft (2010)

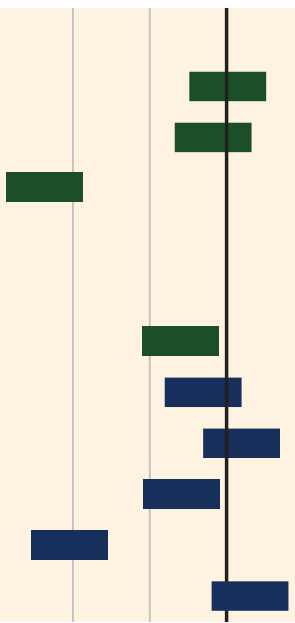

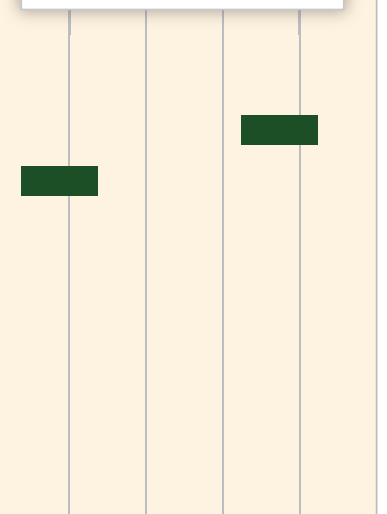
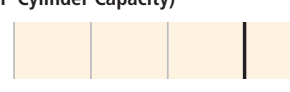

2010 Stock Average 2 Wheeler New Buses, Large Size
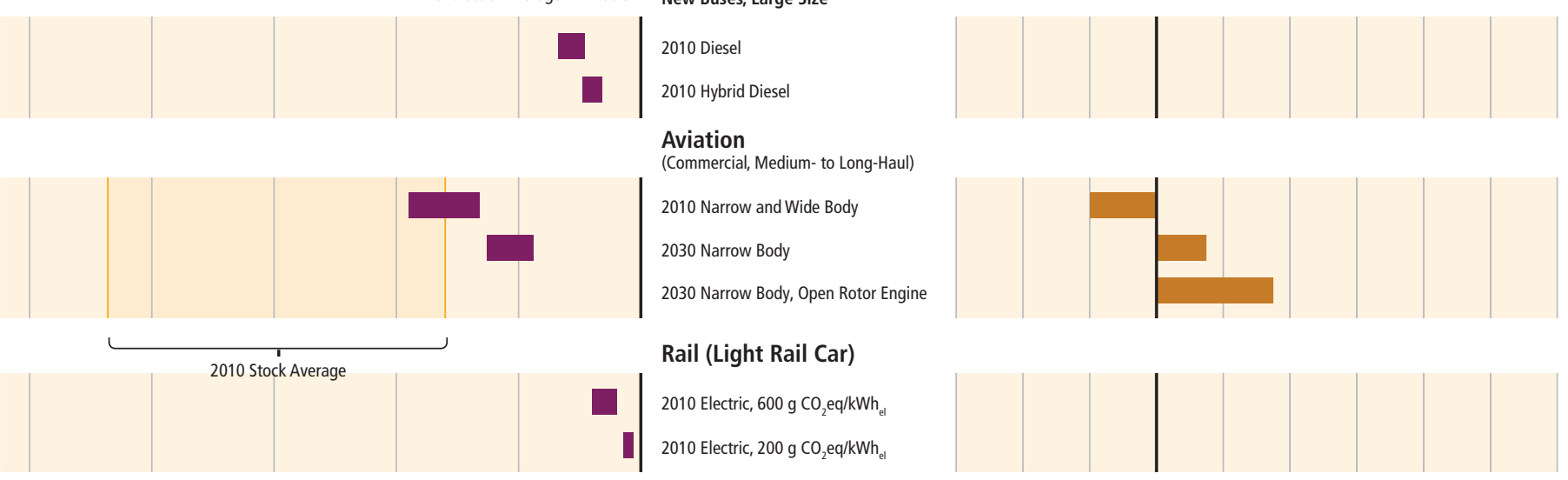

Rail (Light Rail Car)

2010 Electric, $600 \mathrm{~g} \mathrm{CO}_{2}$ eq $/ \mathrm{kWh}$ e
2010 Electric, $200 \mathrm{~g} \mathrm{CO}_{2}$ eq $/ \mathrm{kWh}$

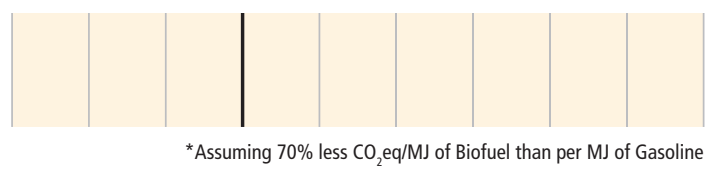

Figure TS.21 | Indicative emissions intensity ( $\left(\mathrm{CCO}_{2} \mathrm{eq} / \mathrm{p}-\mathrm{km}\right)$ and levelized costs of conserved carbon ( $\mathrm{LCCC}$ in $\mathrm{USD}_{2010} / \mathrm{tCO}_{2}$ eq saved) of selected passenger transport technologies. Variations in emissions intensities stem from variation in vehicle efficiencies and occupancy rates. Estimated LCCC for passenger road transport options are point estimates \pm 100 $\mathrm{USD}_{2010} / \mathrm{tCO}_{2}$ eq based on central estimates of input parameters that are very sensitive to assumptions (e. g., specific improvement in vehicle fuel economy to 2030, specific biofuel $\mathrm{CO}_{2}$ eq intensity, vehicle costs, fuel prices). They are derived relative to different baselines (see legend for colour coding) and need to be interpreted accordingly. Estimates for 2030 are based on projections from recent studies, but remain inherently uncertain. LCCC for aviation are taken directly from the literature. Table 8.3 provides additional context (see Annex III.3 for data and assumptions on emissions intensities and cost calculations and Annex II.3.1 for methodological issues on levelized cost metrics). WACC: Weighted average cost of capital. [Table 8.3] 


\section{Freight Transport}

\section{Currently Commercially Available and Future (2030) Expected Technologies}

Emissions Intensity ( $\left.\mathrm{gCO}_{2} \mathrm{eq} / \mathrm{t}-\mathrm{km}\right)$
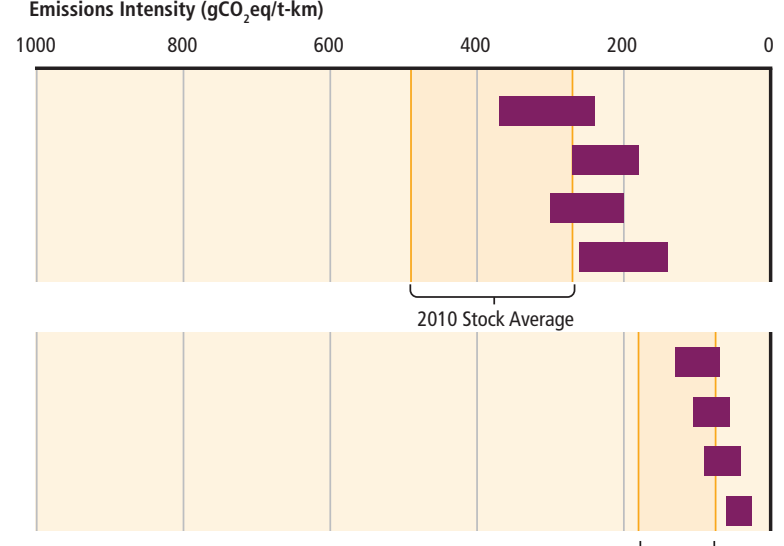

2010 Stock Average
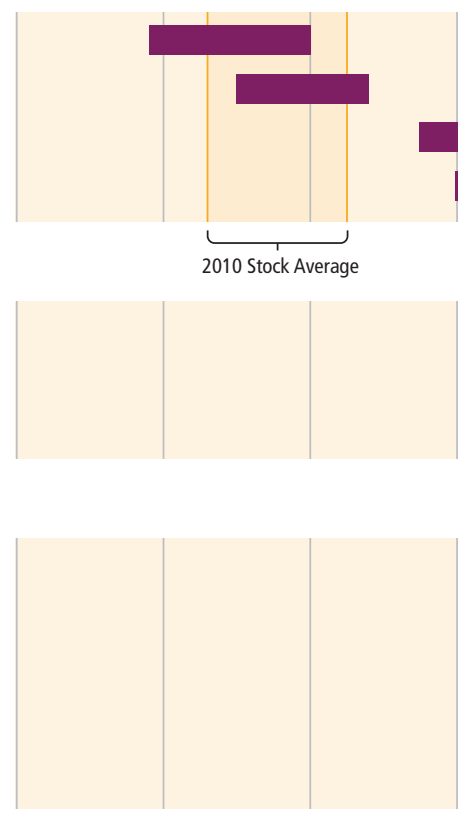
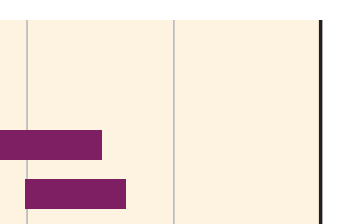

$$
\begin{array}{|l}
\text { Av } \\
20 \\
20 \\
2030 \\
2030
\end{array}
$$

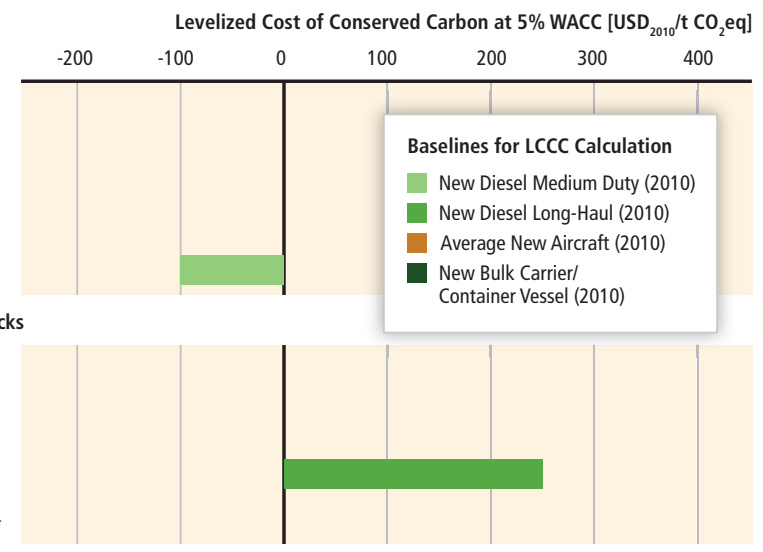

2030 Diesel/Biofuel (50/50 Share) *

\section{Aviation}

(Commercial, Medium- to Long-Haul)

2010 Dedicated Airfreighter

2010 Belly-Hold

2030 Improved Aircraft

2030 Improved, Open Rotor Engine

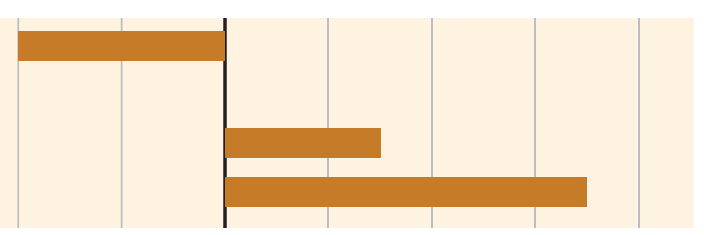

\section{Rail (Freight Train)}

2010 Diesel, Light Goods

2010 Diesel, Heavy Goods

2010 Electric, $200 \mathrm{~g} \mathrm{CO}_{2}$ eq $/ \mathrm{kWh}_{\mathrm{el}}$
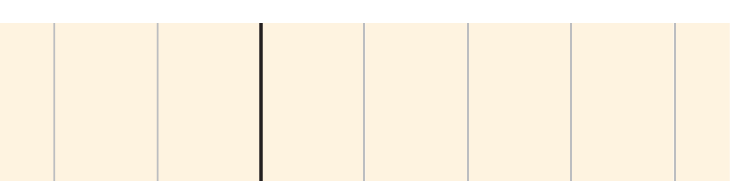

\section{Waterborne}
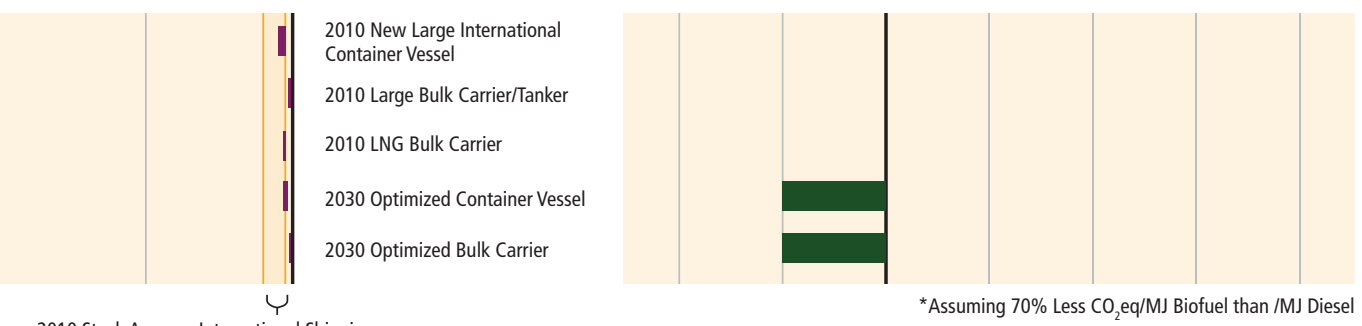

*Assuming $70 \%$ Less $\mathrm{CO}_{2}$ eq/MJ Biofuel than $/ \mathrm{MJ}$ Diesel

Figure TS.22 Indicative emissions intensity ( $\mathrm{tCO}_{2}$ eq/t-km) and levelized costs of conserved carbon (LCCC in USD ${ }_{2010} / \mathrm{tCO}_{2}$ eq saved) of selected freight transport technologies. Variations in emissions intensities largely stem from variation in vehicle efficiencies and load rates. Levelized costs of conserved carbon are taken directly from the literature and are very sensitive to assumptions (e. g., specific improvement in vehicle fuel economy to 2030, specific biofuel $\mathrm{CO}_{2}$ eq intensity, vehicle costs, and fuel prices). They are expressed relative to current baseline technologies (see legend for colour coding) and need to be interpreted accordingly. Estimates for 2030 are based on projections from recent studies but remain inherently uncertain. Table 8.3 provides additional context (see Annex III.3 for data and assumptions on emissions intensities and cost calculations and Annex II.3.1 for methodological issues on levelized cost metrics). LNG: Liquefied natural gas; WACC: Weighted average cost of capital. [Table 8.3] 

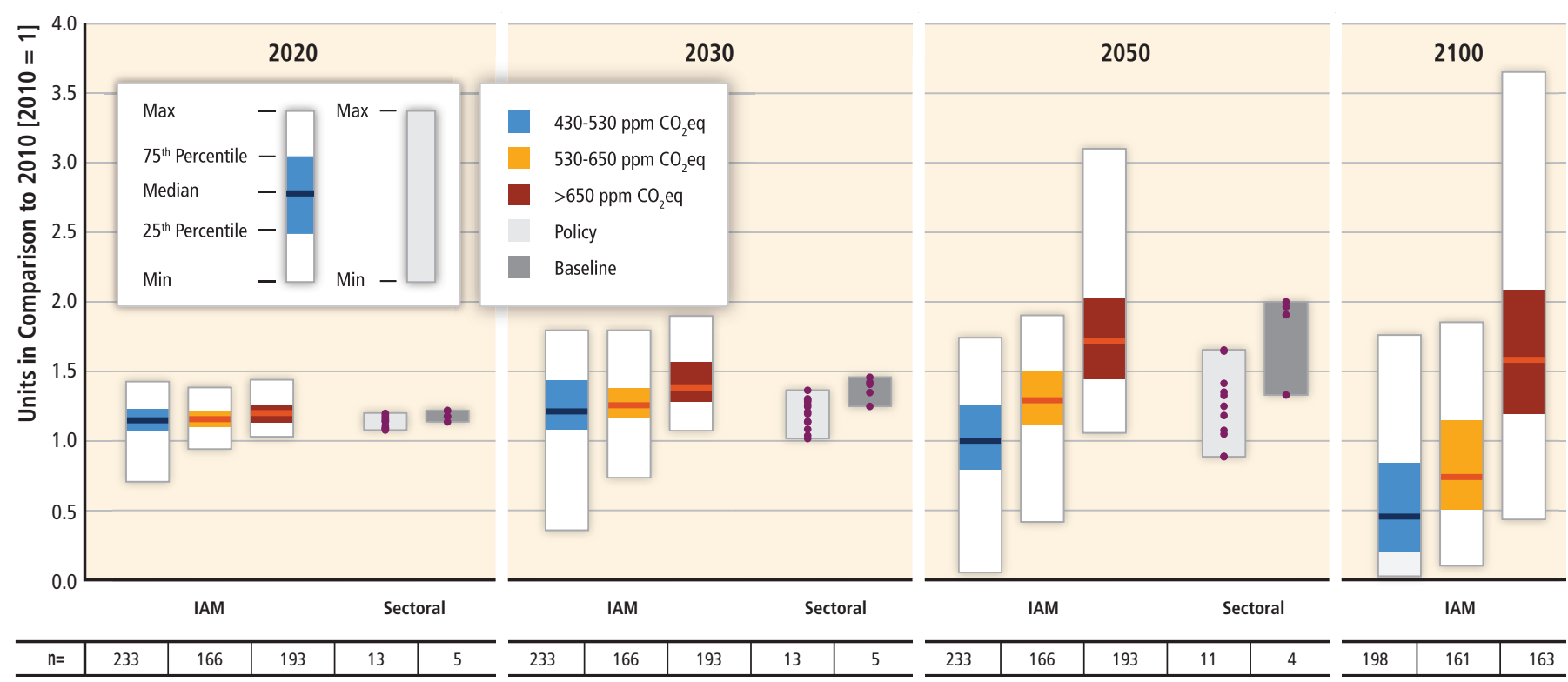

Figure TS.23 Direct global $\mathrm{CO}_{2}$ emissions from all passenger and freight transport are indexed relative to 2010 values for each scenario with integrated model studies grouped by $\mathrm{CO}_{2}$ eq concentration levels by 2100, and sectoral studies grouped by baseline and policy categories. [Figure 8.9]

Table TS.5 | Overview of potential co-benefits (green arrows) and adverse side-effects (orange arrows) of the main mitigation measures in the transport sector; arrows pointing up/down denote a positive/negative effect on the respective objective or concern; a question mark (?) denotes an uncertain net effect. Co-benefits and adverse side-effects depend on local circumstances as well as on implementation practice, pace and scale. For possible upstream effects of low-carbon electricity, see Table TS.4. For possible upstream effects of biomass supply, see Table TS.8. For an assessment of macroeconomic, cross-sectoral effects associated with mitigation policies (e.g., on energy prices, consumption, growth, and trade), see e. g., Sections 3.9, 6.3.6, 13.2.2.3 and 14.4.2. The uncertainty qualifiers in brackets denote the level of evidence and agreement on the respective effects (see TS.1). Abbreviations for evidence: $I=$ limited, $m=$ medium, $r=$ robust; for agreement: $I=$ low, $m=$ medium, $h=$ high. [Table 8.4]

\begin{tabular}{|c|c|c|c|c|c|c|}
\hline \multirow{2}{*}{ Transport } & \multicolumn{6}{|c|}{ Effect on additional objectives/concerns } \\
\hline & & Economic & & Social & & Environmental \\
\hline $\begin{array}{l}\text { Reduction of fuel } \\
\text { carbon intensity: } \\
\text { electricity, } \\
\text { hydrogen }\left(\mathrm{H}_{2}\right) \text {, } \\
\text { compressed natural } \\
\text { gas (CNG), biofuels, } \\
\text { and other fuels }\end{array}$ & $\uparrow$ & $\begin{array}{l}\text { Energy security (diversification, } \\
\text { reduced oil dependence and exposure } \\
\text { to oil price volatility) (m/m) } \\
\text { Technological spillovers (e. g., battery } \\
\text { technologies for consumer electronics) (I/l) }\end{array}$ & $\begin{array}{l}? \\
\downarrow \\
\uparrow\end{array}$ & $\begin{array}{l}\text { Health impact via urban air pollution by } \\
\text { CNG, biofuels: net effect unclear ( } \mathbf{m} / \mathbf{l}) \\
\text { Electricity, } \mathrm{H}_{2} \text { : reducing most pollutants }(\mathbf{r} / \mathbf{h}) \\
\text { Shift to diesel: potentially } \\
\text { increasing pollution }(\mathbf{I} / \mathbf{m}) \\
\text { Health impact via reduced noise } \\
\text { (electricity and fuel cell LDVs) (I/m) } \\
\text { Road safety (silent electric LDVs at low speed) (I/I) }\end{array}$ & ? & $\begin{array}{l}\text { Ecosystem impact of electricity } \\
\text { and hydrogen via } \\
\text { Urban air pollution }(\mathbf{m} / \mathbf{m}) \\
\text { Material use (unsustainable } \\
\text { resource mining) (I/l) } \\
\text { Ecosystem impact of biofuels: see AFOLU }\end{array}$ \\
\hline $\begin{array}{l}\text { Reduction of } \\
\text { energy intensity }\end{array}$ & $\uparrow$ & $\begin{array}{l}\text { Energy security (reduced oil dependence } \\
\text { and exposure to oil price volatility) }(\mathbf{m} / \mathbf{m})\end{array}$ & $\uparrow$ & $\begin{array}{l}\text { Health impact via reduced urban air pollution }(\mathbf{r} / \mathbf{h}) \\
\text { Road safety (via increased crash-worthiness) }(\mathbf{m} / \mathbf{m})\end{array}$ & $\downarrow$ & $\begin{array}{l}\text { Ecosystem and biodiversity impact via } \\
\text { reduced urban air pollution }(\mathbf{m} / \mathbf{h})\end{array}$ \\
\hline $\begin{array}{l}\text { Compact urban } \\
\text { form and improved } \\
\text { transport } \\
\text { infrastructure } \\
\text { Modal shift }\end{array}$ & $\uparrow$ & $\begin{array}{l}\text { Energy security (reduced oil dependence } \\
\text { and exposure to oil price volatility) }(\mathbf{m} / \mathbf{m}) \\
\text { Productivity (reduced urban congestion } \\
\text { and travel times, affordable and } \\
\text { accessible transport) }(\mathbf{m} / \mathbf{h}) \\
\text { Employment opportunities in the public } \\
\text { transport sector vs. car manufacturing }(\mathbf{l} / \mathbf{m})\end{array}$ & $\begin{array}{l}\downarrow \\
\uparrow \\
\downarrow\end{array}$ & $\begin{array}{l}\text { Health impact for non-motorized modes via } \\
\text { Increased physical activity }(\mathbf{r} / \mathbf{h}) \\
\text { Potentially higher exposure to air pollution }(\mathbf{r} / \mathbf{h}) \\
\text { Noise (modal shift and travel reduction) }(\mathbf{r} / \mathbf{h}) \\
\text { Equitable mobility access to } \\
\text { employment opportunities, particularly } \\
\text { in developing countries ( } \mathbf{r} / \mathbf{h}) \\
\text { Road safety (via modal shift and/or infrastructure } \\
\text { for pedestrians and cyclists) ( } \mathbf{r} / \mathbf{h})\end{array}$ & $\downarrow$ & $\begin{array}{l}\text { Ecosystem impact via } \\
\text { Urban air pollution }(\mathbf{r} / \mathbf{h}) \\
\text { Land-use competition }(\mathbf{m} / \mathbf{m})\end{array}$ \\
\hline $\begin{array}{l}\text { Journey distance } \\
\text { reduction and } \\
\text { avoidance }\end{array}$ & $\uparrow$ & $\begin{array}{l}\text { Energy security (reduced oil dependence } \\
\text { and exposure to oil price volatility) }(\mathbf{r} / \mathbf{h}) \\
\text { Productivity (reduced urban congestion, } \\
\text { travel times, walking) }(\mathbf{r} / \mathbf{h})\end{array}$ & $\downarrow$ & $\begin{array}{l}\text { Health impact (for non-motorized } \\
\text { transport modes) (r/h) }\end{array}$ & & $\begin{array}{l}\text { Ecosystem impact via } \\
\quad \text { Urban air pollution }(\mathbf{r} / \mathbf{h}) \\
\quad \text { New/shorter shipping routes }(\mathbf{r} / \mathbf{h}) \\
\text { Land-use competition from } \\
\text { transport infrastructure }(\mathbf{r} / \mathbf{h})\end{array}$ \\
\hline
\end{tabular}




\section{TS.3.2.4 Buildings}

GHG emissions from the buildings sector ${ }^{15}$ have more than doubled since 1970, accounting for $19 \%$ of global GHG emissions in 2010, including indirect emissions from electricity generation. The share rises to $25 \%$ if AFOLU emissions are excluded from the total. The buildings sector also accounted for $32 \%$ of total global final energy use, approximately one-third of black carbon emissions, and an eighth to a third of F-gases, with significant uncertainty (medium evidence, medium agreement). (Figure TS.3) [9.2]

Direct and indirect $\mathrm{CO}_{2}$ emissions from buildings are projected to increase from $8.8 \mathrm{GtCO}_{2} / \mathrm{yr}$ in 2010 to $13-17 \mathrm{GtCO}_{2} / \mathrm{yr}$ in 2050 (25-75th percentile; full range $7.9-22 \mathrm{GtCO}_{2} / \mathrm{yr}$ ) in baseline scenarios; most of the baseline scenarios assessed in WGIII AR5 show a significant increase (medium evidence, medium agreement) (Figure TS.15) [6.8]. The lower end of the full range is dominated by scenarios with a focus on energy intensity improvements that go well beyond the observed improvements over the past 40 years. Without further policies, final energy use of the buildings sector may grow from approximately 120 exajoules per year (EJ/yr) in 2010 to $270 \mathrm{EJ} / \mathrm{yr}$ in 2050 [9.9].

Significant lock-in risks arise from the long lifespans of buildings and related infrastructure (robust evidence, high agreement). If only currently planned policies are implemented, the final energy use in buildings that could be locked-in by 2050, compared to a scenario where today's best practice buildings become the standard in newly built structures and retrofits, is equivalent to approximately $80 \%$ of the final energy use of the buildings sector in 2005. [9.4]

Improvements in wealth, lifestyle change, the provision of access to modern energy services and adequate housing, and urbanization will drive the increases in building energy demand (robust evidence, high agreement). The manner in which those without access to adequate housing (about 0.8 billion people), modern energy carriers, and sufficient levels of energy services including clean cooking and heating (about 3 billion people) meet these needs will influence the development of building-related emissions. In addition, migration to cities, decreasing household size, increasing levels of wealth, and lifestyle changes, including increasing dwelling size and number and use of appliances, all contribute to considerable increases in building energy services demand. The substantial amount of new construction taking place in developing countries represents both a risk and opportunity from a mitigation perspective. $[9.2,9.4,9.9]$

Recent advances in technologies, know-how, and policies in the buildings sector, however, make it feasible that the global total sector final energy use stabilizes or even declines by mid-century (robust evidence, medium agreement). Recent advances in technology,

\footnotetext{
The buildings sector covers the residential, commercial, public and services sectors emissions from construction are accounted for in the industry sector.
}

design practices and know-how, coupled with behavioural changes, can achieve a two to ten-fold reduction in energy requirements of individual new buildings and a two to four-fold reduction for individual existing buildings largely cost-effectively or sometimes even at net negative costs (see Box TS.12) (robust evidence, high agreement). [9.6]

Advances since AR4 include the widespread demonstration worldwide of very low, or net zero energy buildings both in new construction and retrofits (robust evidence, high agreement). In some jurisdictions, these have already gained important market shares with, for instance, over 25 million $\mathrm{m}^{2}$ of building floorspace in Europe complying with the 'Passivehouse' standard in 2012. However, zero energy/carbon buildings may not always be the most cost-optimal solution, nor even be feasible in certain building types and locations. [9.3]

High-performance retrofits are key mitigation strategies in countries with existing building stocks, as buildings are very long-lived and a large fraction of 2050 developed country buildings already exists (robust evidence, high agreement). Reductions of heating/cooling energy use by $50-90 \%$ have been achieved using best practices. Strong evidence shows that very low-energy construction and retrofits can be economically attractive. [9.3]

With ambitious policies it is possible to keep global building energy use constant or significantly reduce it by mid-century compared to baseline scenarios which anticipate an increase of more than two-fold (medium evidence, medium agreement) (Figure TS.24). Detailed building sector studies indicate a larger energy savings potential by 2050 than do integrated studies. The former indicate a potential of up to $70 \%$ of the baseline for heating and cooling only, and around $35-45 \%$ for the whole sector. In general, deeper reductions are possible in thermal energy uses than in other energy services mainly relying on electricity. With respect to additional fuel switching as compared to baseline, both sectoral and integrated studies find modest opportunities. In general, both sectoral and integrated studies indicate that electricity will supply a growing share of building energy demand over the long term, especially if heating demand decreases due to a combination of efficiency gains, better architecture, and climate change. [6.8.4, 9.8.2, Figure 9.19]

The history of energy efficiency programmes in buildings shows that $25-30 \%$ efficiency improvements have been available at costs substantially lower than those of marginal energy supply (robust evidence, high agreement). Technological progress enables the potential for cost-effective energy efficiency improvements to be maintained, despite continuously improving standards. There has been substantial progress in the adoption of voluntary and mandatory standards since AR4, including ambitious building codes and targets, voluntary construction standards, and appliance standards. At the same time, in both new and retrofitted buildings, as well as in appliances and information, communication and media technology equipment, there have been notable performance and cost improvements. Large 


\section{Box TS.12 | Negative private mitigation costs}

A persistent issue in the analysis of mitigation options and costs is whether there are mitigation opportunities that are privately beneficial-generating private benefits that more than offset the costs of implementation-but which consumers and firms do not voluntarily undertake. There is some evidence of unrealized mitigation opportunities that would have negative private cost. Possible examples include investments in vehicles [8.1], lighting and heating technology in homes and commercial buildings [9.3], as well as industrial processes [10.1].

Examples of negative private costs imply that firms and individuals do not take opportunities to save money. This might be explained in a number of ways. One is that status-quo bias can inhibit the switch to new technologies or products [2.4, 3.10.1]. Another is that firms and individuals may focus on short-term goals and discount future costs and benefits sharply; consumers have been shown to do this when choosing energy conservation measures or investing in energy-efficient technologies [2.4.3, 2.6.5.3, 3.10.1]. Risk aversion and ambiguity aversion may also account for this behaviour when outcomes are uncertain [2.4.3, 3.10.1]. Other possible explanations include: insufficient information on opportunities to conserve energy; asymmetric information-for example, landlords may be unable to convey the value of energy efficiency improvements to renters; split incentives, where one party pays for an investment but another party reaps the benefits; and imperfect credit markets, which make it difficult or expensive to obtain finance for energy savings [3.10.1, 16.4].

Some engineering studies show a large potential for negative-cost mitigation. The extent to which such negative-cost opportunities can actually be realized remains a matter of contention in the literature. Empirical evidence is mixed. [Box 3.10]
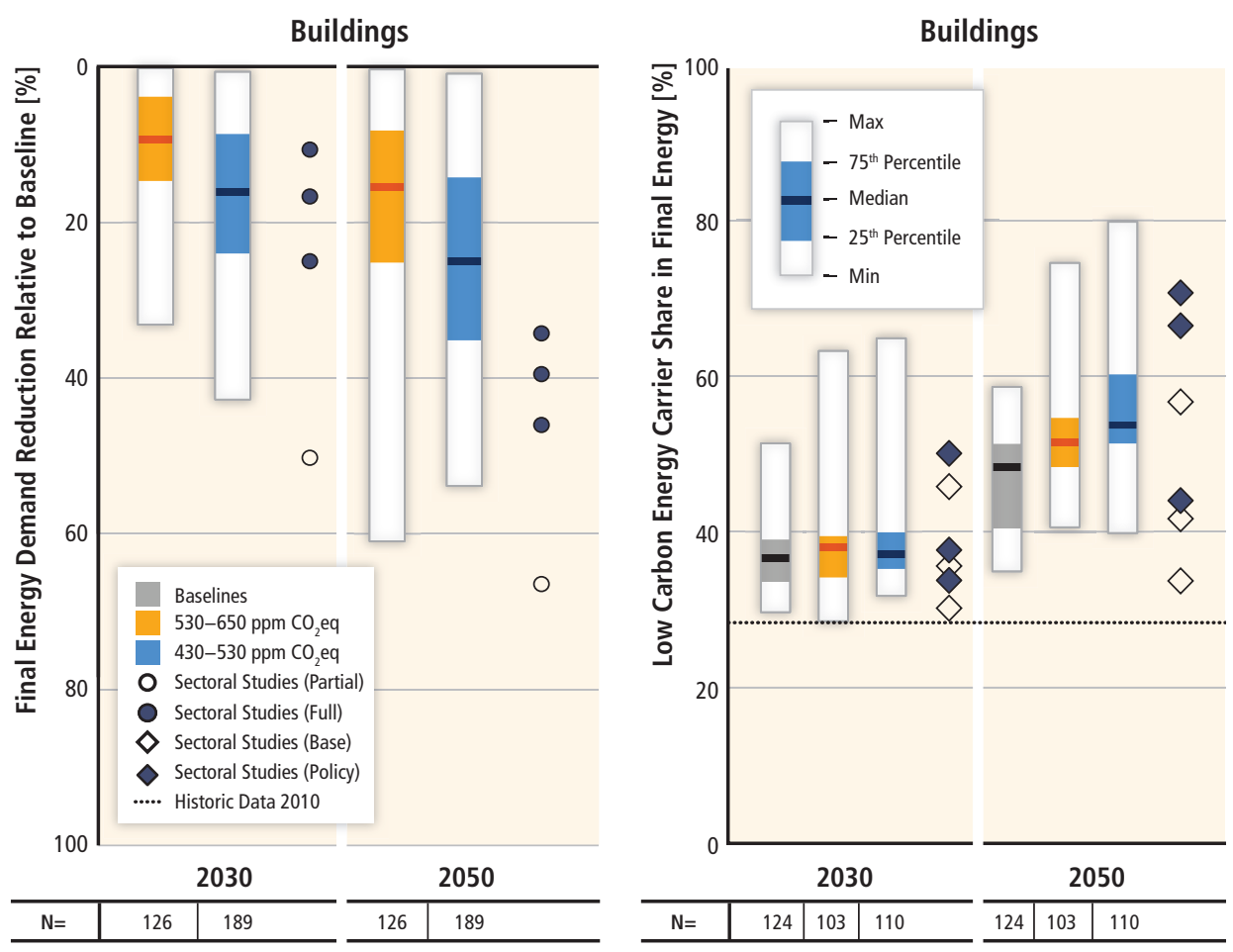

Figure TS.24| Final energy demand reduction relative to baseline (left panel) and development of final low-carbon energy carrier share in final energy (from electricity; right panel) in buildings by 2030 and 2050 in mitigation scenarios from three different $\mathrm{CO}_{2}$ eq concentrations ranges shown in boxplots (see Section 6.3.2) compared to sectoral studies shown in shapes assessed in Chapter 9. Filled circles correspond to sectoral studies with full sectoral coverage while empty circles correspond to studies with only partial sectoral coverage (e. g., heating and cooling). [Figures 6.37 and 6.38]

reductions in thermal energy use in buildings are possible at costs lower than those of marginal energy supply, with the most cost-effective options including very high-performance new commercial buildings; the same holds for efficiency improvements in some appliances and cooking equipment. $[9.5,9.6,9.9]$
Lifestyle, culture, and other behavioural changes may lead to further large reductions in building and appliance energy requirements beyond those achievable through technologies and architecture. A three- to five-fold difference in energy use has been shown for provision of similar building-related energy 
service levels in buildings. (limited evidence, high agreement) For developed countries, scenarios indicate that lifestyle and behavioural changes could reduce energy demand by up to $20 \%$ in the short term and by up to $50 \%$ of present levels by mid-century (medium evidence, medium agreement). There is a high risk that emerging countries follow the same path as developed economies in terms of buildingrelated architecture, lifestyle, and behaviour. But the literature suggests that alternative development pathways exist that provide high levels of building services at much lower energy inputs, incorporating strategies such as learning from traditional lifestyles, architecture, and construction techniques. [9.3]

Most mitigation options in the building sector have considerable and diverse co-benefits (robust evidence, high agreement). These include, but are not limited to: energy security; less need for energy subsidies; health and environmental benefits (due to reduced indoor and outdoor air pollution); productivity and net employment gains; the alleviation of fuel poverty; reduced energy expenditures; increased value for building infrastructure; and improved comfort and services. (Table TS.6) $[9.6,9.7]$
Especially strong barriers in this sector hinder the marketbased uptake of cost-effective technologies and practices; as a consequence, programmes and regulation are more effective than pricing instruments alone (robust evidence, high agreement). Barriers include imperfect information and lack of awareness, principal/agent problems and other split incentives, transaction costs, lack of access to financing, insufficient training in all construction-related trades, and cognitive/behavioural barriers. In developing countries, the large informal sector, energy subsidies, corruption, high implicit discount rates, and insufficient service levels are further barriers. Therefore, market forces alone are not expected to achieve the necessary transformation without external stimuli. Policy intervention addressing all stages of the building and appliance lifecycle and use, plus new business and financial models, are essential. $[9.8,9.10]$

A large portfolio of building-specific energy efficiency policies was already highlighted in AR4, but further considerable advances in available instruments and their implementation have occurred since (robust evidence, high agreement). Evidence shows that many building energy efficiency policies worldwide have

Table TS.6 | Overview of potential co-benefits (green arrows) and adverse side-effects (orange arrows) of the main mitigation measures in the buildings sector; arrows pointing up/down denote a positive/negative effect on the respective objective or concern. Co-benefits and adverse side-effects depend on local circumstances as well as on implementation practice, pace and scale. For possible upstream effects of fuel switching and RE, see Tables TS.4 and TS.8. For an assessment of macroeconomic, cross-sectoral effects associated with mitigation policies (e. g., on energy prices, consumption, growth, and trade), see e.g., Sections 3.9, 6.3.6, 13.2.2.3 and 14.4.2. The uncertainty qualifiers in brackets denote the level of evidence and agreement on the respective effects (see TS.1). Abbreviations for evidence: $I=$ limited, $m=$ medium, $r=$ robust; for agreement: $I=l o w, m=$ medium, $h=$ high. [Table 9.7]

\begin{tabular}{|c|c|c|c|c|c|c|c|}
\hline \multirow{2}{*}{ Buildings } & \multicolumn{7}{|c|}{ Effect on additional objectives/concerns } \\
\hline & & Economic & & Social & & Environmental & Other \\
\hline $\begin{array}{l}\text { Fuel } \\
\text { switching, RES } \\
\text { incorporation, } \\
\text { green roofs, } \\
\text { and other } \\
\text { measures } \\
\text { reducing GHG } \\
\text { emissions } \\
\text { intensity }\end{array}$ & $\uparrow$ & $\begin{array}{l}\text { Energy security }(\mathbf{m} / \mathbf{h}) \\
\text { Employment impact }(\mathbf{m} / \mathbf{m}) \\
\text { Lower need for energy subsidies }(\mathrm{I} / \mathrm{I}) \\
\text { Asset values of buildings }(\mathrm{I} / \mathbf{m})\end{array}$ & $\begin{array}{l}\downarrow \\
\uparrow \\
\downarrow \\
\uparrow\end{array}$ & $\begin{array}{l}\text { Fuel poverty (residential) via } \\
\quad \text { Energy demand }(\mathbf{m} / \mathbf{h}) \\
\quad \text { Energy cost }(\mathbf{I} / \mathbf{m}) \\
\text { Energy access (for higher } \\
\text { energy cost) }(\mathbf{I} / \mathbf{m}) \\
\text { Productive time for women/children (for } \\
\text { replaced traditional cookstoves) }(\mathbf{m} / \mathbf{h})\end{array}$ & & $\begin{array}{l}\text { Health impact in residential buildings via } \\
\text { Outdoor air pollution }(\mathbf{r} / \mathbf{h}) \\
\text { Indoor air pollution (in } \\
\text { developing countries) }(\mathbf{r} / \mathbf{h}) \\
\text { Fuel poverty }(\mathbf{r} / \mathbf{h}) \\
\text { Ecosystem impact (less outdoor } \\
\text { air pollution) }(\mathbf{r} / \mathbf{h}) \\
\text { Urban biodiversity (for } \\
\text { green roofs) }(\mathbf{m} / \mathbf{m})\end{array}$ & $\begin{array}{l}\text { Reduced Urban Heat } \\
\text { Island (UHI) effect (I/m) }\end{array}$ \\
\hline $\begin{array}{l}\text { Retrofits } \\
\text { of existing } \\
\text { buildings } \\
\text { (e.g., cool } \\
\text { roof, passive } \\
\text { solar, etc.) } \\
\text { Exemplary new } \\
\text { buildings } \\
\text { Efficient } \\
\text { equipment }\end{array}$ & $\mid$ & $\begin{array}{l}\text { Energy security }(\mathbf{m} / \mathbf{h}) \\
\text { Employment impact }(\mathbf{m} / \mathbf{m}) \\
\text { Productivity (for commercial } \\
\text { buildings) }(\mathbf{m} / \mathbf{h}) \\
\text { Lower need for energy subsidies (I/I) } \\
\text { Asset values of buildings }(\mathbf{I} / \mathbf{m}) \\
\text { Disaster resilience }(\mathbf{I} / \mathbf{m})\end{array}$ & $\begin{array}{l}\downarrow \\
\downarrow \\
\uparrow \\
\uparrow\end{array}$ & $\begin{array}{l}\text { Fuel poverty (for retrofits and } \\
\text { efficient equipment) (m/h) } \\
\text { Energy access (higher cost for housing } \\
\text { due to the investments needed) }(\mathbf{I} / \mathbf{m}) \\
\text { Thermal comfort (for retrofits and } \\
\text { exemplary new buildings) }(\mathbf{m} / \mathbf{h}) \\
\text { Productive time for women } \\
\text { and children (for replaced } \\
\text { traditional cookstoves) }(\mathbf{m} / \mathbf{h})\end{array}$ & $\begin{array}{l}\downarrow \\
\downarrow \\
\downarrow \\
\downarrow \\
\downarrow \\
\downarrow \\
\downarrow\end{array}$ & $\begin{array}{l}\text { Health impact via } \\
\text { Outdoor air pollution }(\mathbf{r} / \mathbf{h}) \\
\text { Indoor air pollution (for } \\
\text { efficient cookstoves) }(\mathbf{r} / \mathbf{h}) \\
\text { Improved indoor environmental } \\
\text { conditions }(\mathbf{m} / \mathbf{h}) \\
\text { Fuel poverty }(\mathbf{r} / \mathbf{h}) \\
\text { Insufficient ventilation }(\mathbf{m} / \mathbf{m}) \\
\text { Ecosystem impact (less outdoor } \\
\text { air pollution) }(\mathbf{r} / \mathbf{h}) \\
\text { Water consumption and } \\
\text { sewage production (I/I) }\end{array}$ & $\begin{array}{l}\text { Reduced UHI effect } \\
\text { (for retrofits and } \\
\text { new exemplary } \\
\text { buildings) (I/m) }\end{array}$ \\
\hline $\begin{array}{l}\text { Behavioural } \\
\text { changes } \\
\text { reducing } \\
\text { energy demand }\end{array}$ & & $\begin{array}{l}\text { Energy security }(\mathbf{m} / \mathbf{h}) \\
\text { Lower need for energy subsidies (I/l) }\end{array}$ & & & & $\begin{array}{l}\text { Health impact via less outdoor air } \\
\text { pollution }(\mathbf{r} / \mathbf{h}) \text { and improved indoor } \\
\text { environmental conditions }(\mathbf{m} / \mathbf{h}) \\
\text { Ecosystem impact (less outdoor } \\
\text { air pollution) }(\mathbf{r} / \mathbf{h})\end{array}$ & \\
\hline
\end{tabular}


already been saving GHG emissions at large negative costs. Among the most environmentally and cost-effective policies are regulatory instruments such as building and appliance energy performance standards and labels, as well as public leadership programmes and procurement policies. Progress in building codes and appliance standards in some developed countries over the last decade have contributed to stabilizing or even reducing total building energy use, despite growth in population, wealth, and corresponding energy service level demands. Developing countries have also been adopting different effective policies, most notably appliance standards. However, in order to reach ambitious climate goals, these standards need to be substantially strengthened and adopted in further jurisdictions, and to other building and appliance types. Due to larger capital requirements, financing instruments are essential both in developed and developing countries to achieve deep reductions in energy use. [9.10]

\section{TS.3.2.5 Industry}

In 2010, the industry sector accounted for around $28 \%$ of final energy use, and direct and indirect GHG emissions (the latter being associated with electricity consumption) are larger than the emissions from either the buildings or transport end-use sectors and represent just over $30 \%$ of global GHG emissions in 2010 (the share rises to $40 \%$ if AFOLU emissions are excluded from the total) (high confidence). Despite the declining share of industry in global GDP, global industry and waste/wastewater GHG emissions grew from $10 \mathrm{GtCO}_{2}$ eq in 1990 to $13 \mathrm{GtCO}_{2}$ eq in 2005 and to $15 \mathrm{GtCO}_{2}$ eq in 2010 (of which waste/wastewater accounted for $1.4 \mathrm{GtCO}_{2}$ eq). [10.3]

Carbon dioxide emissions from industry, including direct and indirect emissions as well as process emissions, are projected to increase from $13 \mathrm{GtCO}_{2} / \mathrm{yr}$ in 2010 to $20-24 \mathrm{GtCO}_{2} / \mathrm{yr}$ in 2050 (25-75th percentile; full range $9.5-34 \mathrm{GtCO}_{2} / \mathrm{yr}$ ) in baseline scenarios; most of the baseline scenarios assessed in WGIII AR5 show a significant increase (medium evidence, medium agreement) (Figure TS.15) [6.8]. The lower end of the full range is dominated by scenarios with a focus on energy intensity improvements that go well beyond the observed improvements over the past 40 years.

The wide-scale upgrading, replacement and deployment of best available technologies, particularly in countries where these are not in practice, and in non-energy intensive industries, could directly reduce the energy intensity of the industry sector by about $25 \%$ compared to the current level (robust evidence, high agreement). Despite long-standing attention to energy efficiency in industry, many options for improved energy efficiency still remain. Through innovation, additional reductions of about $20 \%$ in energy intensity may potentially be realized (limited evidence, medium agree-

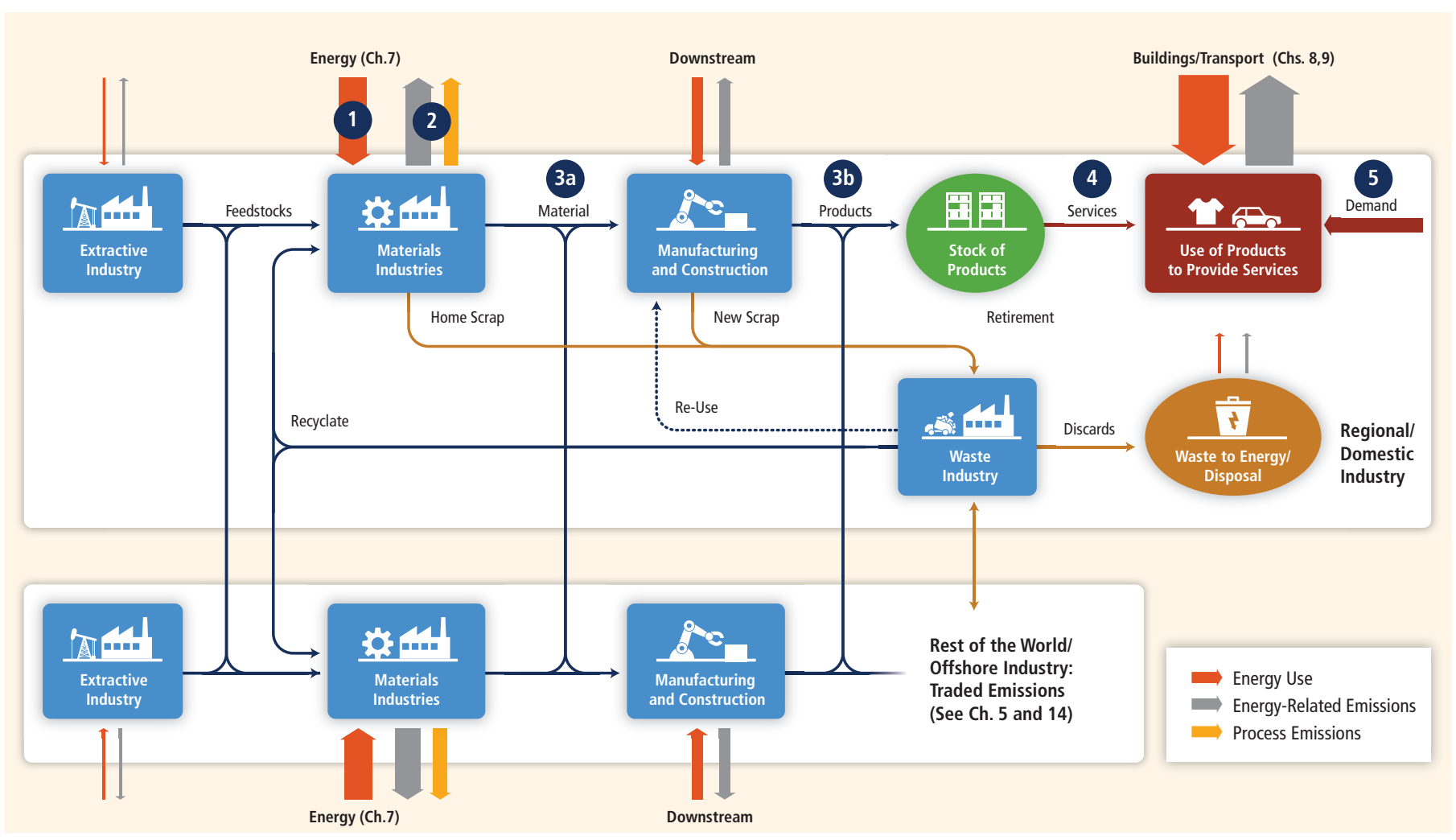

Figure TS.25| A schematic illustration of industrial activity over the supply chain. Options for mitigation in the industry sector are indicated by the circled numbers: (1) energy efficiency; (2) emissions efficiency; (3a) material efficiency in manufacturing; (3b) material efficiency in product design; (4) product-service efficiency; (5) service demand reduction. [Figure 10.2] 
ment). Barriers to implementing energy efficiency relate largely to the initial investment costs and lack of information. Information programmes are a prevalent approach for promoting energy efficiency, followed by economic instruments, regulatory approaches, and voluntary actions. [10.4, 10.7, 10.9, 10.11]

An absolute reduction in emissions from the industry sector will require deployment of a broad set of mitigation options that go beyond energy efficiency measures (medium evidence, high agreement) [10.4, 10.7]. In the context of continued overall growth in industrial demand, substantial reductions from the sector will require parallel efforts to increase emissions efficiency (e.g., through fuel and feedstock switching or CCS); material use efficiency (e.g., less scrap, new product design); recycling and re-use of materials and products; product-service efficiency (e.g., more intensive use of products through car sharing, longer life for products); radical product innovations (e.g., alternatives to cement); as well as service demand reductions. Lack of policy and experiences in material and product-service efficiency are major barriers. (Table TS.3, Figure TS.25) [10.4, 10.7, 10.11]

While detailed industry sector studies tend to be more conservative than integrated studies, both identify possible industrial final energy demand savings of around $30 \%$ by 2050 in mitigation scenarios not exceeding 650 ppm $\mathrm{CO}_{2} \mathrm{eq}$ by 2100 relative to baseline scenarios (medium evidence, medium agreement) (Figure TS.26). Integrated models in general treat the industry sector in a more aggregated fashion and mostly do not explicitly provide detailed sub-sectoral material flows, options for reducing material demand, and price-induced inter-input substitution possibilities. Due to the heterogeneous character of the industry sector, a coherent comparison between sectoral and integrated studies remains difficult. [6.8.4, 10.4, 10.7, 10.10.1, Figure 10.14]

Mitigation in the industry sector can also be achieved by reducing material and fossil fuel demand by enhanced waste use, which concomitantly reduces direct GHG emissions from waste disposal (robust evidence, high agreement). The hierarchy of waste management places waste reduction at the top, followed by re-use, recycling, and energy recovery. As the share of recycled or reused material is still low, applying waste treatment technologies and recovering energy to reduce demand for fossil fuels can result in direct emission reductions from waste disposal. Globally, only about $20 \%$ of municipal solid waste (MSW) is recycled and about $14 \%$ is treated with energy recovery while the rest is deposited in open dumpsites or landfills. About $47 \%$ of wastewater produced in the domestic and manufacturing sectors is still untreated. The largest cost range is for reducing GHG emissions from landfilling through the treatment of waste by anaerobic digestion. The costs range from negative (see Box TS.12) to very high. Advanced wastewater treatment technologies may enhance GHG emissions reduction in wastewater treatment but they are clustered among the higher cost options (medium evidence, medium agreement). (Figure TS.29) [10.4, 10.14]
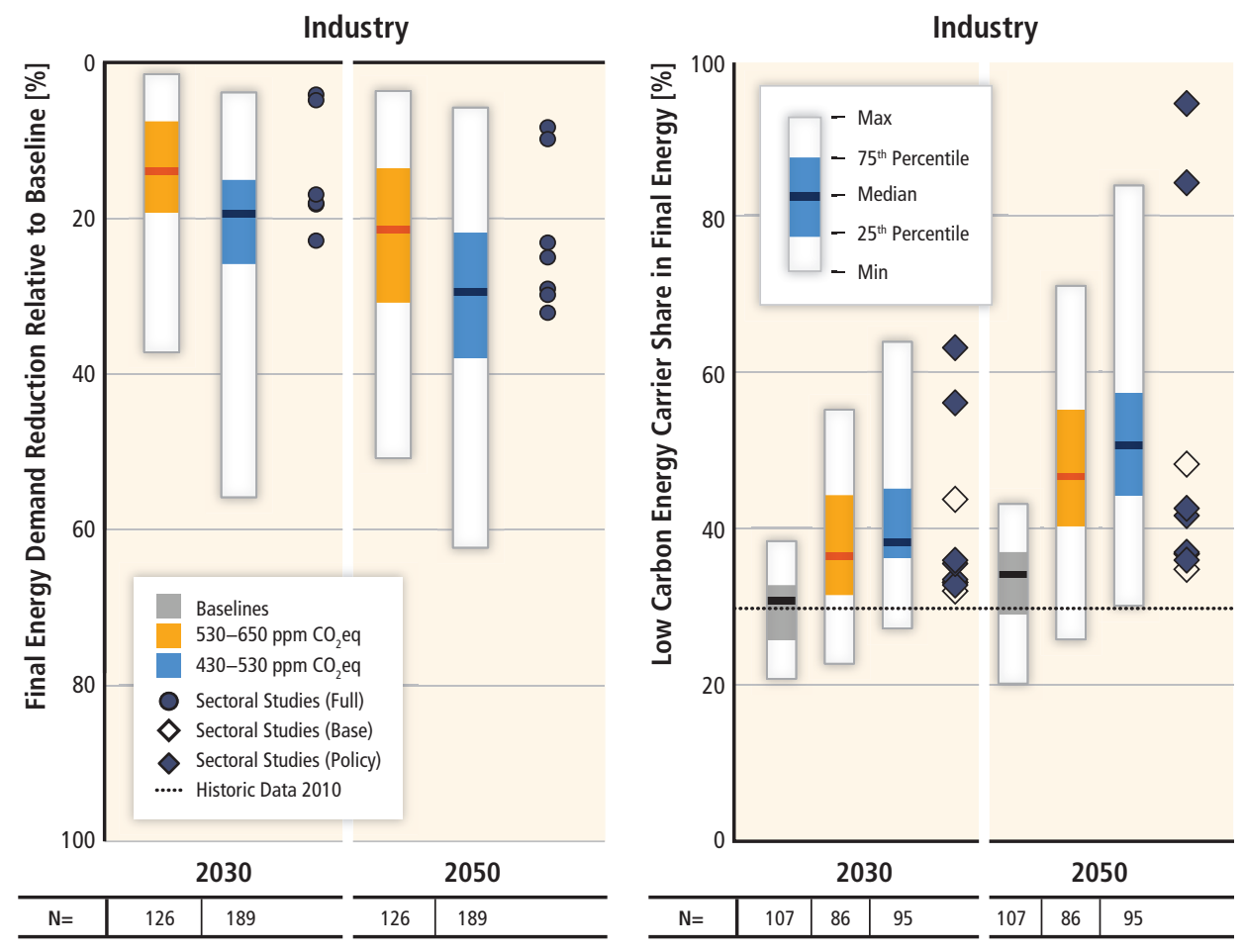

Figure TS.26| Final energy demand reduction relative to baseline (left panel) and development of final low-carbon energy carrier share in final energy (including electricity, heat, hydrogen, and bioenergy; right panel) in industry by 2030 and 2050 in mitigation scenarios from three different $\mathrm{CO}_{2}$ eq concentration ranges shown in boxplots (see Section 6.3.2) compared to sectoral studies shown in shapes assessed in Chapter 10. Filled circles correspond to sectoral studies with full sectoral coverage. [Figures 6.37 and 6.38] 
Scenarios Reaching 450 ppm CO 2 eq in 2100 in Integrated Models

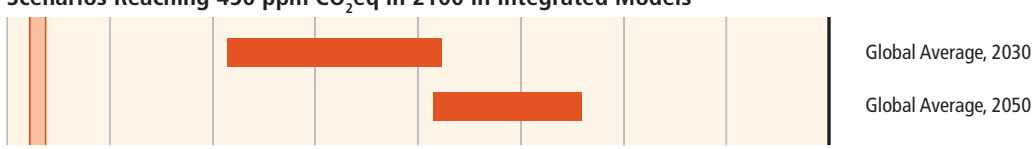

Currently Commercially Available Technologies
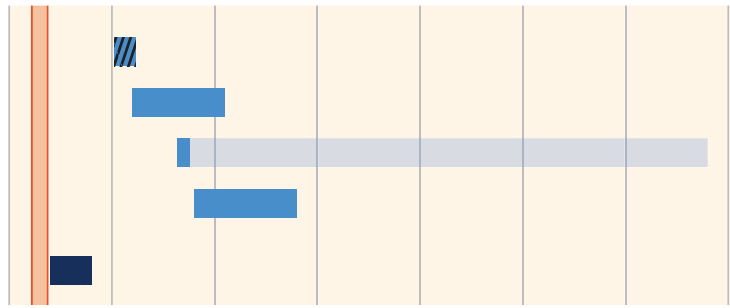

Best Practice Energy Intensity

Best Practice Clinker Substitution

Improvements in Non-Electric Fuel Mix

Best Practice Energy Intensity and Clinker

Substitution Combined

Decarbonization of Electricity Supply

Technologies in Pre-Commercial Stage

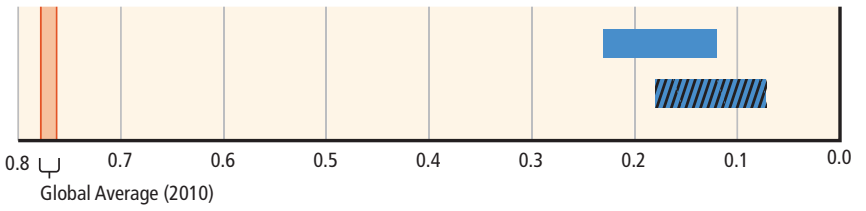

Emission Intensity $\left[\mathrm{tCO}_{2} / \mathrm{t}\right.$ Cement]
CCS

CCS and Fully Decarbonized Electricity Supply Combined
Data from Integrated Models
Effect from Increased Use of Biomass as Non-Electric Fuel*

Scenarios Reaching 450 ppm $\mathrm{CO}_{2}$ eq in 2100 in Integrated Models

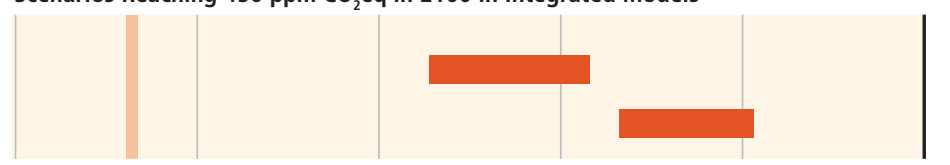

Currently Commercially Available Technologies

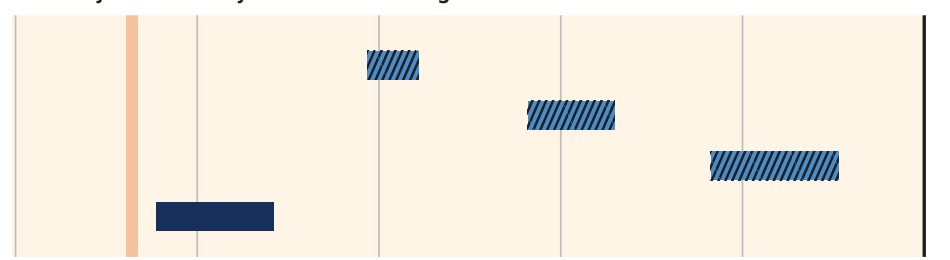

Technologies in Pre-Commercial Stage

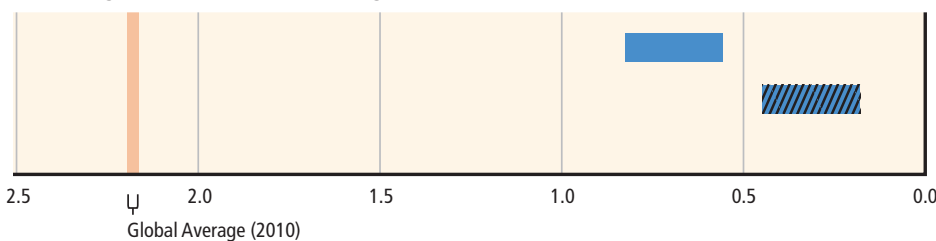

Emission Intensity $\left[\mathrm{tCO}_{2} / \mathrm{t}\right.$ Crude Steel]
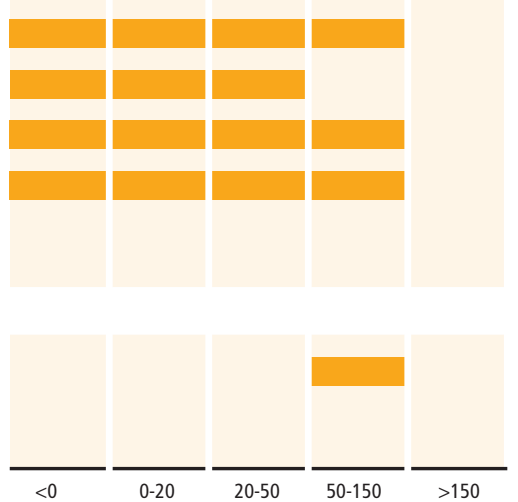

Indicative Cost of Conserved Carbon[USD ${ }_{2010} / \mathrm{tCO}_{2}$ ]

Global Average (2030)

Global Average (2050)

Advanced Blast Furnace Route

Natural Gas DRI Route

Scrap Based EAF

Decarbonization of

Electricity Supply

CCS

CCS and Fully Decarbonized

Electricity Supply Combined

* Assuming for Simplicity that Biomass Burning is Carbon Neutral

Data from Integrated Models

Measure Affects Direct Emissions

Measure Affects Indirect Emissions

III. Measure Affects Direct and Indirect Emissions

Figure TS.27| Indicative $\mathrm{CO}_{2}$ emission intensities for cement (upper panel) and steel (lower panel) production, as well as indicative levelized cost of conserved carbon (LCCC) shown for various production practices/technologies and for $450 \mathrm{ppm} \mathrm{CO}_{2}$ eq scenarios of a limited selection of integrated models (for data and methodology, see Annex III). DRI: Direct reduced iron; EAF: Electric arc furnace. [Figures 10.7, 10.8]

Waste policy and regulation have largely influenced material consumption, but few policies have specifically pursued material efficiency or product-service efficiency (robust evidence, high agreement) [10.11]. Barriers to improving material efficiency include lack of human and institutional capacities to encourage management decisions and public participation. Also, there is a lack of experience and often there are no clear incentives either for suppliers or consumers to address improvements in material or product-service efficiency, or to reduce product demand. [10.9]

$\mathrm{CO}_{2}$ emissions dominate GHG emissions from industry, but there are also substantial mitigation opportunities for non- $\mathrm{CO}_{2}$ gases 
IEA ETP 2DS Scenario
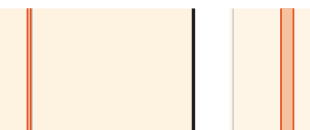

I

Currently Commercially Available Technologies

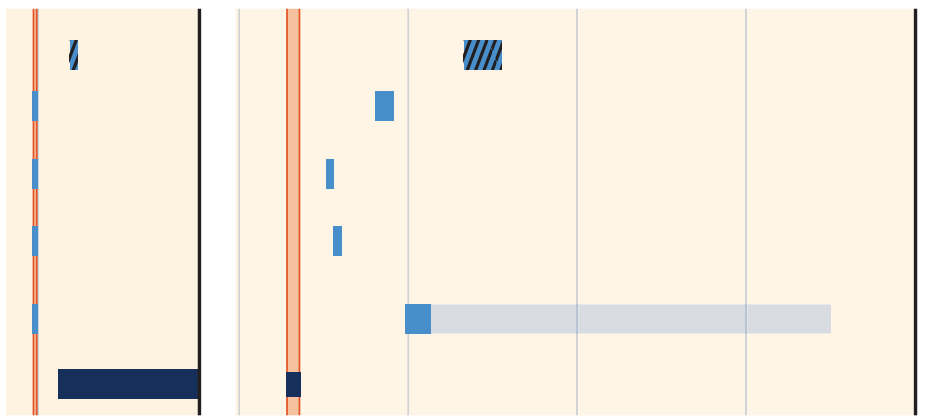

Technologies in Pre-Commercial Stage
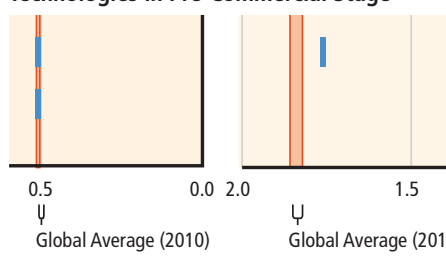

५

Global Average (2010)

Indirect Emissions Direct Emissions [ $\left.\mathrm{GtCO}_{2} \mathrm{eq}\right]$

[ $\left.\mathrm{GtCO}_{2} \mathrm{eq}\right]$
Global Total (2030)

Global Total (2050)

Best Practice Energy Intensity

Enhanced Recycling, Cogeneration and Process Intensification

Abatement of $\mathrm{N}_{2} \mathrm{O}$ from Nitric and Adipic Acid

Abatement of HFC-23 Emissions from HFC-22 Production

Improvements in Non-Electric Fuel Mix

Decarbonization of Electricity Supply

CCS for Ammonia Production

CCS Applied to Non-Electric Fuel-Related Emissions

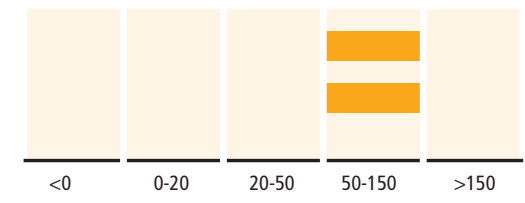

Indicative Cost of Conserved Carbon[USD $\left.{ }_{2010} / \mathrm{tCO}_{2} \mathrm{eq}\right]$

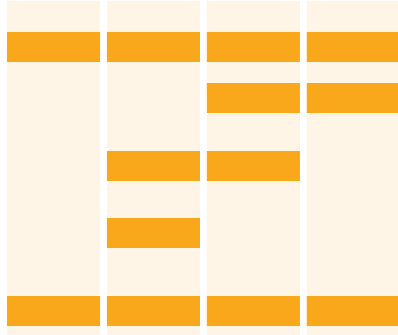

Data from Integrated Models

Effect from Increased Use of Biomass as Non-Electric Fuel*
Measure Affects Direct Emissions

Measure Affects Indirect Emissions

\section{IEA ETP 2DS Scenario}

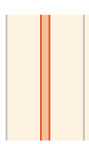

Currently Commercially Available Technologies

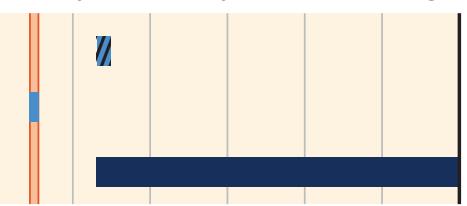

Technologies in Pre-Commercial Stage

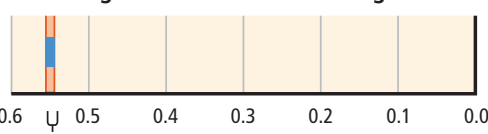

Global Average (2010)

Indirect Emission Intensity $\left[\mathrm{tCO}_{2} / \mathrm{t}\right.$ Paper]

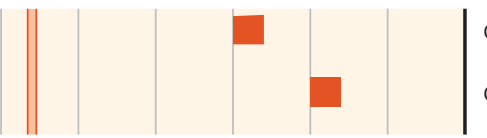

Global Average (2030)

Global Average (2050)
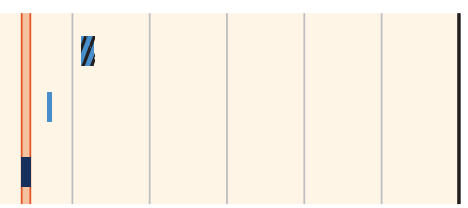

Best Practice Energy Intensity

Cogeneration

Decarbonization of Electricity Supply

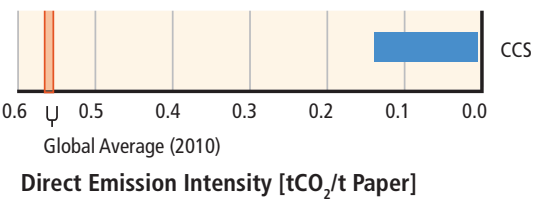

CCS

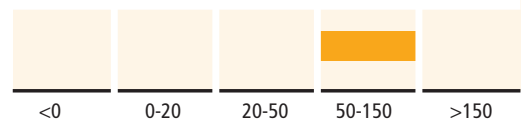

Indicative Cost of Conserved Carbon[ $\left.\mathrm{USD}_{2010} / \mathrm{tCO}_{2}\right]$

Data from Integrated Models

Measure Affects Direct Emissions

Measure Affects Indirect Emissions

III. Measure Affects Direct and Indirect Emissions

Figure TS.28 | Indicative global $\mathrm{CO}_{2}$ eq emissions for chemicals production (upper panel) and indicative global $\mathrm{CO}_{2}$ emission intensities for paper production (lower panel) as well as indicative levelized cost of conserved carbon (LCCC) shown for various production practices/technologies and for $450 \mathrm{ppm} \mathrm{CO}_{2}$ eq scenarios of a limited selection of integrated models (for data and methodology, see Annex III). [Figures 10.9, 10.10] 

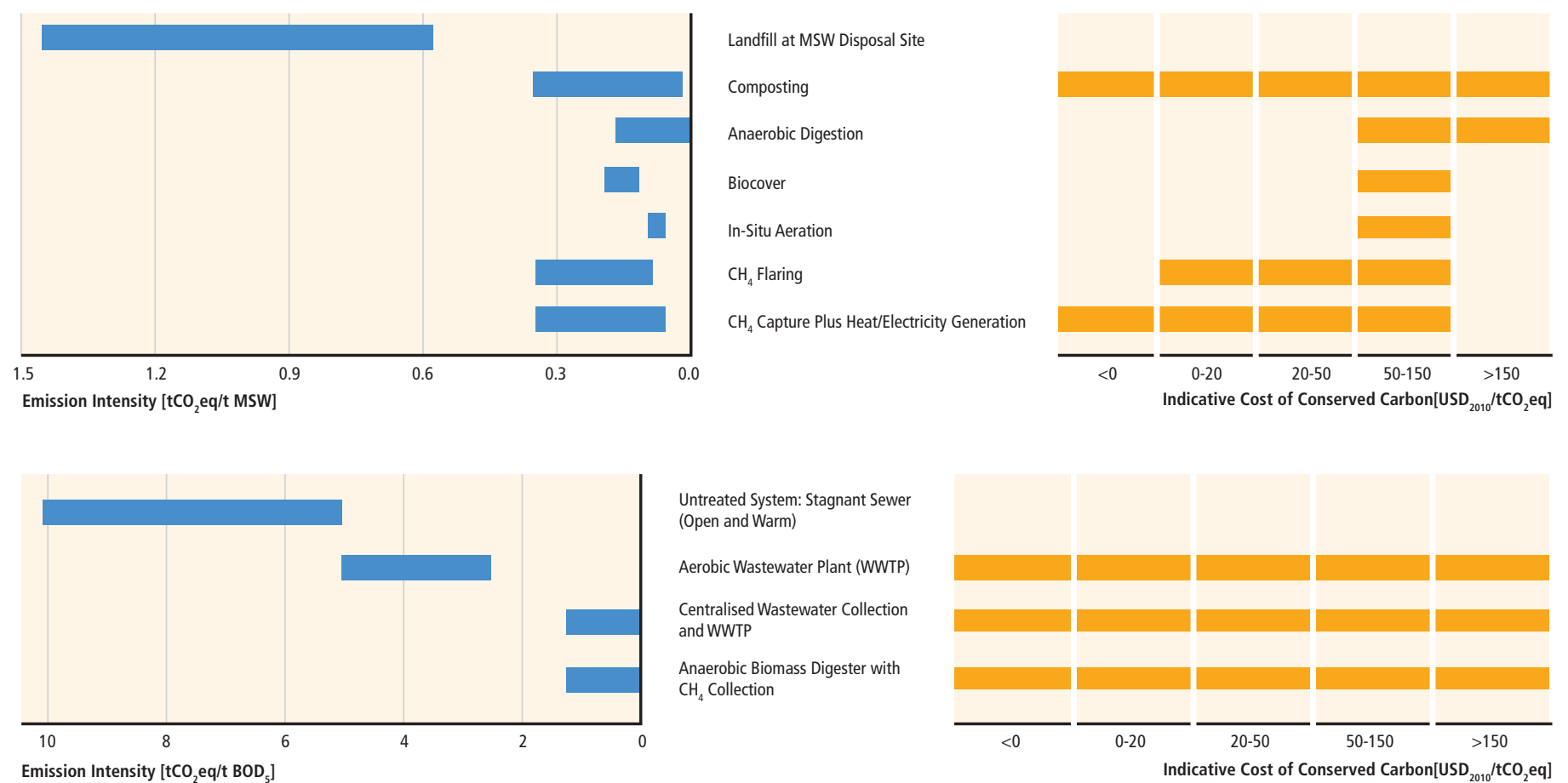

Figure TS.29| Indicative $\mathrm{CO}_{2}$ eq emission intensities for waste (upper panel) and wastewater (lower panel) of various practices as well as indicative levelized cost of conserved carbon (for data and methodology, see Annex III). MSW: Municipal solid waste. [Figures 10.19 and 10.20]

(robust evidence, high agreement). Methane $\left(\mathrm{CH}_{4}\right)$, nitrous oxide $\left(\mathrm{N}_{2} \mathrm{O}\right)$ and fluorinated gases (F-gases) from industry accounted for emissions of $0.9 \mathrm{GtCO}_{2}$ eq in 2010. Key mitigation opportunities comprise, e. g., reduction of hydrofluorocarbon (HFC) emissions by leak repair, refrigerant recovery and recycling, and proper disposal and replacement by alternative refrigerants (ammonia, $\mathrm{HC}_{1} \mathrm{CO}_{2}$ ). $\mathrm{N}_{2} \mathrm{O}$ emissions from adipic and nitric acid production can be reduced through the implementation of thermal destruction and secondary catalysts. The reduction of non- $\mathrm{CO}_{2}$ GHGs also faces numerous barriers. Lack of awareness, lack of economic incentives and lack of commercially available technologies (e. g., for HFC recycling and incineration) are typical examples. [Table 10.2, 10.7]

Systemic approaches and collaborative activities across companies (large energy-intensive industries and Small and Medium Enterprises (SMEs)) and sectors can help to reduce GHG emissions (robust evidence, high agreement). Cross-cutting technologies such as efficient motors, and cross-cutting measures such as reducing air or steam leaks, help to optimize performance of industrial processes and improve plant efficiency very often cost-effectively with both energy savings and emissions benefits. Industrial clusters also help to realize mitigation, particularly from SMEs. [10.4] Cooperation and cross-sectoral collaboration at different levels_-for example, sharing of infrastructure, information, waste heat, cooling, etc.—may provide further mitigation potential in certain regions/industry types [10.5].

Several emission-reducing options in the industrial sector are cost-effective and profitable (medium evidence, medium agreement). While options in cost ranges of $0-20$ and $20-50 \mathrm{USD} / \mathrm{tCO}$ eq and even below 0 USD/ $\mathrm{tCO}_{2}$ eq exist, achieving near-zero emissions intensity levels in the industry sector would require the additional realization of long-term step-change options (e. g., CCS), which are associated with higher levelized costs of conserved carbon (LCCC) in the range of 50-150 USD/tCO ${ }_{2}$ eq. Similar cost estimates for implementing material efficiency, product-service efficiency, and service demand reduction strategies are not available. With regard to long-term options, some sector-specific measures allow for significant reductions in specific GHG emissions but may not be applicable at scale, e.g., scrapbased iron and steel production. Decarbonized electricity can play an important role in some subsectors (e.g., chemicals, pulp and paper, and aluminium), but will have limited impact in others (e. g., cement, iron and steel, waste). In general, mitigation costs vary regionally and depend on site-specific conditions. (Figures TS.27, TS.28, TS.29) [10.7]

\section{Mitigation measures are often associated with co-benefits (robust} evidence, high agreement). Co-benefits include enhanced competitiveness through cost-reductions, new business opportunities, better environmental compliance, health benefits through better local air and water quality and better work conditions, and reduced waste, all of which provide multiple indirect private and social benefits (Table TS.7). [10.8]

There is no single policy that can address the full range of mitigation measures available for industry and overcome associated barriers. Unless barriers to mitigation in industry are resolved, the pace and extent of mitigation in industry will be limited and even profitable measures will remain untapped (robust evidence, high agreement). [10.9, 10.11] 
Table TS.7 | Overview of potential co-benefits (green arrows) and adverse side-effects (orange arrows) of the main mitigation measures in the industry sector; arrows pointing up/down denote a positive/negative effect on the respective objective or concern. Co-benefits and adverse side-effects depend on local circumstances as well as on the implementation practice, pace and scale. For possible upstream effects of low-carbon energy supply (includes CCS), see Table TS.4. For possible upstream effects of biomass supply, see Table TS.8. For an assessment of macroeconomic, cross-sectoral, effects associated with mitigation policies (e. g., on energy prices, consumption, growth, and trade), see e. g., Sections 3.9, 6.3.6, 13.2.2.3 and 14.4.2. The uncertainty qualifiers in brackets denote the level of evidence and agreement on the respective effects (see TS.1). Abbreviations for evidence: I = limited, $\mathrm{m}=$ medium, $\mathrm{r}=$ robust; for agreement: $\mathrm{I}=$ low, $\mathrm{m}=$ medium, $\mathrm{h}=$ high. [Table 10.5]

\begin{tabular}{|c|c|c|c|c|c|c|}
\hline \multirow{2}{*}{ Industry } & \multicolumn{6}{|c|}{ Effect on additional objectives/concerns } \\
\hline & & Economic & & Social & & Environmental \\
\hline $\begin{array}{l}\mathrm{CO}_{2} \text { and non- } \mathrm{CO}_{2} \\
\mathrm{GHG} \text { emissions } \\
\text { intensity reduction }\end{array}$ & $\uparrow$ & Competitiveness and productivity $(\mathbf{m} / \mathbf{h})$ & & $\begin{array}{l}\text { Health impact via reduced local air } \\
\text { pollution and better work conditions (for } \\
\text { perfluorocarbons from aluminium) }(\mathbf{m} / \mathbf{m})\end{array}$ & $\uparrow$ & $\begin{array}{l}\text { Ecosystem impact via reduced local air } \\
\text { pollution and reduced water pollution }(\mathbf{m} / \mathbf{m}) \\
\text { Water conservation }(\mathbf{I} / \mathbf{m})\end{array}$ \\
\hline $\begin{array}{l}\text { Technical energy } \\
\text { efficiency improvements } \\
\text { via new processes } \\
\text { and technologies }\end{array}$ & $\begin{array}{l}\uparrow \\
\uparrow\end{array}$ & $\begin{array}{l}\text { Energy security (via lower } \\
\text { energy intensity) }(\mathbf{m} / \mathbf{m}) \\
\text { Employment impact }(\mathbf{I} / \mathbf{l}) \\
\text { Competitiveness and productivity }(\mathbf{m} / \mathbf{h}) \\
\text { Technological spillovers in developing } \\
\text { countries (due to supply chain linkages) (I/I) }\end{array}$ & $\begin{array}{l}\downarrow \\
\uparrow \\
\uparrow \\
\uparrow\end{array}$ & $\begin{array}{l}\text { Health impact via reduced } \\
\text { local pollution }(\mathbf{I} / \mathbf{m}) \\
\text { New business opportunities }(\mathbf{m} / \mathbf{m}) \\
\text { Water availability and quality }(\mathbf{I} / \mathbf{I}) \\
\text { Safety, working conditions and } \\
\text { job satisfaction }(\mathbf{m} / \mathbf{m})\end{array}$ & $\begin{array}{l}\downarrow \\
\downarrow\end{array}$ & $\begin{array}{l}\text { Ecosystem impact via: } \\
\text { Fossil fuel extraction }(\mathbf{I} / \mathbf{l}) \\
\text { Local pollution and waste }(\mathbf{m} / \mathbf{m})\end{array}$ \\
\hline $\begin{array}{l}\text { Material efficiency } \\
\text { of goods, recycling }\end{array}$ & $\uparrow$ & $\begin{array}{l}\text { National sales tax revenue } \\
\text { in medium term (I/I) } \\
\text { Employment impact in waste } \\
\text { recycling market }(\mathbf{I} / \mathbf{I}) \\
\text { Competitiveness in manufacturing (I/I) } \\
\text { New infrastructure for industrial clusters (I/I) }\end{array}$ & & $\begin{array}{l}\text { Health impacts and safety concerns }(\mathbf{I} / \mathbf{m}) \\
\text { New business opportunities }(\mathbf{m} / \mathbf{m}) \\
\text { Local conflicts (reduced resource } \\
\text { extraction) }(\mathbf{I} / \mathbf{m})\end{array}$ & $\downarrow$ & $\begin{array}{l}\text { Ecosystem impact via reduced local } \\
\text { air and water pollution and waste } \\
\text { material disposal }(\mathbf{m} / \mathbf{m}) \\
\text { Use of raw/virgin materials and } \\
\text { natural resources implying reduced } \\
\text { unsustainable resource mining }(\mathbf{I} / \mathbf{l})\end{array}$ \\
\hline $\begin{array}{l}\text { Product demand } \\
\text { reductions }\end{array}$ & $\downarrow$ & $\begin{array}{l}\text { National sales tax revenue } \\
\text { in medium term }(\mathbf{I} / \mathbf{I})\end{array}$ & & Wellbeing via diverse lifestyle choices (I/I) & $\downarrow$ & Post-consumption waste (I/I) \\
\hline
\end{tabular}

\section{TS.3.2.6 Agriculture, Forestry and Other Land Use (AFOLU)}

Since AR4, GHG emissions from the AFOLU sector have stabilized but the share of total anthropogenic GHG emissions has decreased (robust evidence, high agreement). The average annual total GHG flux from the AFOLU sector was $10-12 \mathrm{GtCO}_{2}$ eq in 2000-2010, with global emissions of 5.0-5.8 $\mathrm{GtCO}_{2} \mathrm{eq} / \mathrm{yr}$ from agriculture on average and around $4.3-5.5 \mathrm{GtCO}_{2}$ eq/yr from forestry and other land uses. Non- $\mathrm{CO}_{2}$ emissions derive largely from agriculture, dominated by $\mathrm{N}_{2} \mathrm{O}$ emissions from agricultural soils and $\mathrm{CH}_{4}$ emissions from livestock enteric fermentation, manure management, and emissions from rice paddies, totalling 5.0-5.8 $\mathrm{GtCO}_{2} \mathrm{eq} / \mathrm{yr}$ in 2010 (robust evidence, high agreement). Over recent years, most estimates of FOLU $\mathrm{CO}_{2}$ fluxes indicate a decline in emissions, largely due to decreasing deforestation rates and increased afforestation (limited evidence, medium agreement). The absolute levels of emissions from deforestation and degradation have fallen from 1990 to 2010 (robust evidence, high agreement). Over the same time period, total emissions for highincome countries decreased while those of low-income countries increased. In general, AFOLU emissions from high-income countries are dominated by agriculture activities while those from low-income countries are dominated by deforestation and degradation. [Figure $1.3,11.2]$
Net annual baseline $\mathrm{CO}_{2}$ emissions from AFOLU are projected to decline over time with net emissions potentially less than half of the 2010 level by 2050, and the possibility of the AFOLU sector becoming a net sink before the end of century. However, the uncertainty in historical net AFOLU emissions is larger than for other sectors, and additional uncertainties in projected baseline net AFOLU emissions exist. (medium evidence, high agreement) (Figure TS.15) [6.3.1.4, 6.8, Figure 6.5] As in AR4, most projections suggest declining annual net $\mathrm{CO}_{2}$ emissions in the long run. In part, this is driven by technological change, as well as projected declining rates of agriculture area expansion related to the expected slowing in population growth. However, unlike AR4, none of the more recent scenarios projects growth in the near-term. There is also a somewhat larger range of variation later in the century, with some models projecting a stronger net sink starting in 2050 (limited evidence, medium agreement). There are few reported projections of baseline global land-related $\mathrm{N}_{2} \mathrm{O}$ and $\mathrm{CH}_{4}$ emissions and they indicate an increase over time. Cumulatively, land $\mathrm{CH}_{4}$ emissions are projected to be $44-53 \%$ of total $\mathrm{CH}_{4}$ emissions through 2030, and $41-59 \%$ through 2100 , and land $\mathrm{N}_{2} \mathrm{O}$ emissions $85-89 \%$ and $85-90 \%$, respectively (limited evidence, medium agreement). [11.9]

Opportunities for mitigation in the AFOLU sector include supply- and demand-side mitigation options (robust evidence, high agreement). Supply-side measures involve reducing emissions arising 
from land-use change, in particular reducing deforestation, and land and livestock management, increasing carbon stocks by sequestration in soils and biomass, or the substitution of fossil fuels by biomass for energy production (Table TS.3). Further new supply-side technologies not assessed in AR4, such as biochar or wood products for energyintensive building materials, could contribute to the mitigation potential of the AFOLU sector, but there are still few studies upon which to make robust estimates. Demand-side measures include dietary change and waste reduction in the food supply chain. Increasing forestry and agricultural production without a commensurate increase in emissions (i.e., one component of sustainable intensification; Figure TS.30) also reduces emissions intensity (i.e., the GHG emissions per unit of product), a mitigation mechanism largely unreported for AFOLU in AR4, which could reduce absolute emissions as long as production volumes do not increase. $[11.3,11.4]$

Among supply-side measures, the most cost-effective forestry options are afforestation, sustainable forest management and reducing deforestation, with large differences in their relative importance across regions; in agriculture, low carbon prices $^{16}$ (20 USD/ $/ \mathrm{CO}_{2}$ eq) favour cropland and grazing land management and high carbon prices (100 USD/tCO ${ }_{2}$ eq) favour restoration of organic soils (medium evidence, medium agreement). When considering only studies that cover both forestry and agriculture and include agricultural soil carbon sequestration, the economic mitigation potential in the AFOLU sector is estimated to be 7.18 to 10.6 (full range of all studies: $0.49-10.6) \mathrm{GtCO}_{2} \mathrm{eq} / \mathrm{yr}$ in 2030 for mitigation efforts consistent with carbon prices up to $100 \mathrm{USD} / \mathrm{tCO}_{2} \mathrm{eq}$, about a third of which can be achieved at $<20 \mathrm{USD} / \mathrm{tCO}_{2}$ eq (medium evidence, medium agreement). The range of global estimates at a given carbon price partly reflects uncertainty surrounding AFOLU mitigation

16 In many models that are used to assess the economic costs of mitigation, carbon price is used as a proxy to represent the level of effort in mitigation policies (see Glossary). potentials in the literature and the land-use assumptions of the scenarios considered. The ranges of estimates also reflect differences in the GHGs and options considered in the studies. A comparison of estimates of economic mitigation potential in the AFOLU sector published since AR4 is shown in Figure TS.31. [11.6]

While demand-side measures are under-researched, changes in diet, reductions of losses in the food supply chain, and other measures have a significant, but uncertain, potential to reduce GHG emissions from food production $\left(0.76-8.55 \mathrm{GtCO}_{2} \mathrm{eq} / \mathrm{yr}\right.$ by 2050) (Figure TS.31) (limited evidence, medium agreement). Barriers to implementation are substantial, and include concerns about jeopardizing health and well-being, and cultural and societal resistance to behavioural change. However, in countries with a high consumption of animal protein, co-benefits are reflected in positive health impacts resulting from changes in diet (robust evidence, high agreement). [11.4.3, 11.6, 11.7, 11.9]

The mitigation potential of AFOLU is highly dependent on broader factors related to land-use policy and patterns (medium evidence, high agreement). The many possible uses of land can compete or work in synergy. The main barriers to mitigation are institutional (lack of tenure and poor governance), accessibility to financing mechanisms, availability of land and water, and poverty. On the other hand, AFOLU mitigation options can promote innovation, and many technological supply-side mitigation options also increase agricultural and silvicultural efficiency, and can reduce climate vulnerability by improving resilience. Multifunctional systems that allow the delivery of multiple services from land have the capacity to deliver to many policy goals in addition to mitigation, such as improving land tenure, the governance of natural resources, and equity [11.8] (limited evidence, high agreement). Recent frameworks, such as those for assessing environmental or ecosystem services, could provide tools for valuing the multiple synergies and tradeoffs that may arise from mitigation actions (Table TS.8) (medium evidence, medium agreement). $[11.7,11.8]$

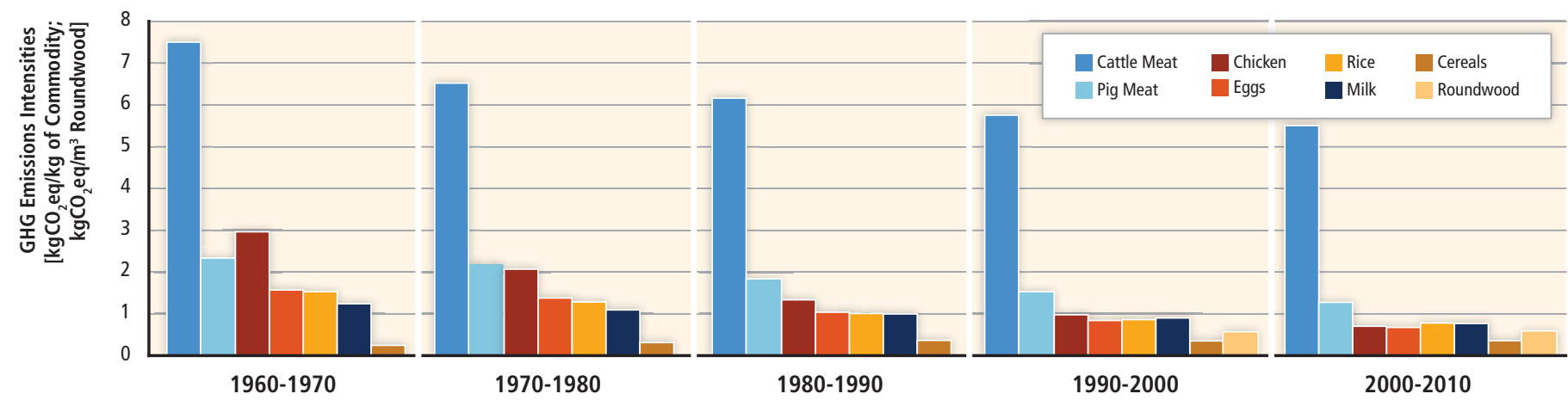

Figure TS.30 | GHG emissions intensities of selected major AFOLU commodities for decades 1960s-2000s. (1) Cattle meat, defined as GHG (enteric fermentation + manure management of cattle, dairy and non-dairy)/meat produced; (2) pig meat, defined as GHG (enteric fermentation + manure management of swine, market and breeding)/meat produced; (3) chicken meat, defined as GHG (manure management of chickens)/meat produced; (4) milk, defined as GHG (enteric fermentation + manure management of cattle, dairy)/milk produced; (5) eggs, defined as GHG (manure management of chickens, layers)/egg produced; (6) rice, defined as GHG (rice cultivation)/rice produced; (7) cereals, defined as GHG (synthetic fertilizers)/cereals produced; (8) wood, defined as GHG (carbon loss from harvest)/roundwood produced. [Figure 11.15] 


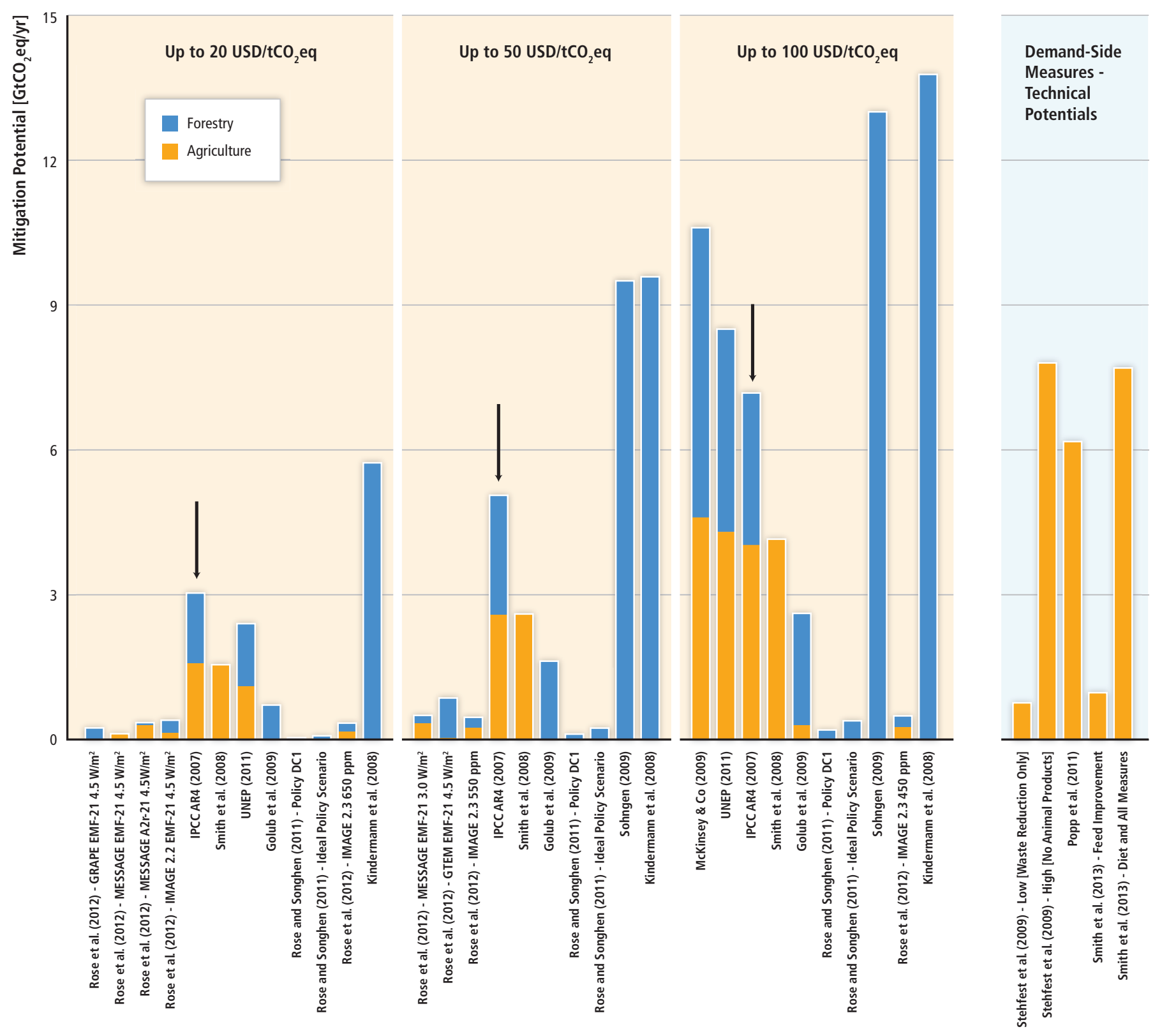

Figure TS.31 | Estimates of economic mitigation potentials in the AFOLU sector published since AR4 (AR4 estimates shown for comparison, denoted by black arrows), including bottom-up, sectoral studies, and top-down, multi-sector studies. Supply-side mitigation potentials are estimated for around 2030, ranging from 2025 to 2035, and are for agriculture, forestry or both sectors combined. Studies are aggregated for potentials up to $\sim 20 \mathrm{USD} / \mathrm{tCO}_{2} \mathrm{eq}$ (actual range 1.64-21.45), up to $\sim 50 \mathrm{USD} / \mathrm{tCO} \mathrm{eq}_{2}$ (actual range 31.39-50.00), and up to $~ 100 \mathrm{USD} / \mathrm{tCO}_{2} \mathrm{eq}$ (actual range 70.0-120.91). Demand-side measures (shown on the right hand side of the figure) are for $\sim 2050$ and are not assessed at a specific carbon price, and should be regarded as technical potentials. Smith et al. (2013) values are the mean of the range. Not all studies consider the same measures or the same GHGs. [11.6.2, Figure 11.14]

Policies governing practices in agriculture as well as forest conservation and management need to account for the needs of both mitigation and adaptation (medium evidence, high agreement). Some mitigation options in the AFOLU sector (such as soil and forest carbon stocks) may be vulnerable to climate change. Economic incentives (e. g., special credit lines for low-carbon agriculture, sustainable agriculture and forestry practices, tradable credits, payment for ecosystem services) and regulatory approaches (e. g., enforcement of environmental law to protect forest carbon stocks by reducing defor- estation, set-aside policies, air and water pollution control reducing nitrate load and $\mathrm{N}_{2} \mathrm{O}$ emissions) have been effective in different cases. Investments in research, development, and diffusion (e. g., increase of resource use-efficiency (fertilizers), livestock improvement, better forestry management practices) could result in synergies between adaptation and mitigation. Successful cases of deforestation reduction in different regions are found to combine different policies such as land planning, regulatory approaches and economic incentives (limited evidence, high agreement). [11.3.2, 11.10, 15.11] 
Table TS.8 | Overview of potential co-benefits (green arrows) and adverse side-effects (orange arrows) of the main mitigation measures in the AFOLU sector; arrows pointing up/down denote a positive/negative effect on the respective objective or concern. These effects depend on the specific context (including bio-physic, institutional and socioeconomic aspects) as well as on the scale of implementation. For an assessment of macroeconomic, cross-sectoral effects associated with mitigation policies (e.g., on energy prices, consumption, growth, and trade), see e.g., Sections 3.9, 6.3.6, 13.2.2.3 and 14.4.2. The uncertainty qualifiers in brackets denote the level of evidence and agreement on the respective effects (see TS.1). Abbreviations for evidence: $\mathrm{I}=$ limited, $\mathrm{m}=$ medium, $\mathrm{r}=$ robust; for agreement: $\mathrm{I}=$ low, $\mathrm{m}=$ medium, $\mathrm{h}=$ high. [Tables 11.9 and 11.12]

\begin{tabular}{|c|c|c|c|c|c|c|c|c|}
\hline \multirow{2}{*}{ AFOLU } & \multicolumn{8}{|c|}{ Effect on additional objectives/concerns } \\
\hline & & Economic & & Social & & Environmental & & Institutional \\
\hline $\begin{array}{l}\text { Supply side: } \\
\text { Forestry, land- } \\
\text { based agriculture, } \\
\text { livestock, } \\
\text { integrated } \\
\text { systems, and } \\
\text { bioenergy } \\
\text { (marked by *) } \\
\text { Demand side: } \\
\text { Reduced losses } \\
\text { in the food } \\
\text { supply chain, } \\
\text { changes in human } \\
\text { diets, changes } \\
\text { in demand } \\
\text { for wood and } \\
\text { forestry products }\end{array}$ & $\begin{array}{l}\star \\
\uparrow \\
\downarrow \\
\downarrow \\
\uparrow * \\
\uparrow *\end{array}$ & $\begin{array}{l}\text { Employment impact via } \\
\text { Entrepreneurship } \\
\text { development ( } \mathbf{m} / \mathbf{h}) \\
\text { Use of less labour- } \\
\text { intensive technologies } \\
\text { in agriculture }(\mathbf{m} / \mathbf{m}) \\
\text { Diversification of income } \\
\text { sources and access } \\
\text { to markets ( } \mathbf{r} / \mathbf{h}) \\
\text { Additional income to } \\
\text { (sustainable) landscape } \\
\text { management ( } \mathbf{m} / \mathbf{h}) \\
\text { Income concentration ( } \mathbf{m} / \mathbf{m}) \\
\text { Energy security (resource } \\
\text { sufficiency) ( } \mathbf{m} / \mathbf{h}) \\
\text { Innovative financing } \\
\text { mechanisms for sustainable } \\
\text { resource management }(\mathbf{m} / \mathbf{h}) \\
\text { Technology innovation } \\
\text { and transfer ( } \mathbf{m} / \mathbf{m})\end{array}$ & $\begin{array}{l}\downarrow * \\
\uparrow \\
\uparrow \\
\uparrow * \\
\uparrow\end{array}$ & $\begin{array}{l}\text { Food-crops production through } \\
\text { integrated systems and sustainable } \\
\text { agriculture intensification ( } \mathbf{r} / \mathbf{m}) \\
\text { Food production (locally) due } \\
\text { to large-scale monocultures } \\
\text { of non-food crops ( } \mathbf{r} / \mathbf{l}) \\
\text { Cultural habitats and recreational } \\
\text { areas via (sustainable) forest } \\
\text { management and conservation ( } \mathbf{m} / \mathbf{m} \text { ) } \\
\text { Human health and animal welfare e.g., } \\
\text { through less pesticides, reduced burning } \\
\text { practices, and practices like agroforestry } \\
\text { and silvo-pastoral systems ( } \mathbf{m} / \mathbf{h} \text { ) } \\
\text { Human health when using } \\
\text { burning practices (in agriculture } \\
\text { or bioenergy) ( } \mathbf{m} / \mathbf{m} \text { ) } \\
\text { Gender, intra- and inter- } \\
\text { generational equity via } \\
\text { Participation and fair } \\
\text { benefit sharing ( } \mathbf{r} / \mathbf{h} \text { ) } \\
\text { Concentration of benefits ( } \mathbf{m} / \mathbf{m} \text { ) }\end{array}$ & $\begin{array}{l}\downarrow * \\
\uparrow * \\
\uparrow \\
\uparrow \\
\downarrow \\
\uparrow \\
\uparrow\end{array}$ & $\begin{array}{l}\text { Provision of ecosystem } \\
\text { services via } \\
\text { Ecosystem } \\
\text { conservation and } \\
\text { sustainable } \\
\text { management as well } \\
\text { as sustainable } \\
\text { agriculture ( } \mathbf{r} / \mathbf{h}) \\
\text { Large scale } \\
\text { monocultures }(\mathbf{r} / \mathbf{h}) \\
\text { Land-use competition }(\mathbf{r} / \mathbf{m}) \\
\text { Soil quality }(\mathbf{r} / \mathbf{h}) \\
\text { Erosion }(\mathbf{r} / \mathbf{h}) \\
\text { Ecosystem resilience }(\mathbf{m} / \mathbf{h}) \\
\text { Albedo and } \\
\text { evaporation }(\mathbf{r} / \mathbf{h})\end{array}$ & $\uparrow \downarrow *$ & $\begin{array}{l}\text { Tenure and use rights } \\
\text { at the local level (for } \\
\text { indigenous people and } \\
\text { local communities) } \\
\text { especially when } \\
\text { implementing activities } \\
\text { in natural forests ( } \mathbf{r} / \mathbf{h}) \\
\text { Access to participative } \\
\text { mechanisms for land } \\
\text { management decisions ( } \mathbf{r} / \mathbf{h} \text { ) } \\
\text { Enforcement of existing } \\
\text { policies for sustainable } \\
\text { resource management }(\mathbf{r} / \mathbf{h})\end{array}$ \\
\hline
\end{tabular}

Reducing Emissions from Deforestation and Forest Degradation $(\text { REDD }+)^{17}$ can be a very cost-effective policy option for mitigating climate change, if implemented in a sustainable manner (limited evidence, medium agreement). REDD+ includes: reducing emissions from deforestation and forest degradation; conservation of forest carbon stocks; sustainable management of forests; and enhancement of forest carbon stocks. It could supply a large share of global abatement of emissions from the AFOLU sector, especially through reducing deforestation in tropical regions, with potential economic, social and other environmental co-benefits. To assure these co-benefits, the implementation of national REDD+ strategies would need to consider financing mechanisms to local stakeholders, safeguards (such as land rights, conservation of biodiversity and other natural resources), and the appropriate scale and institutional capacity for monitoring and verification. [11.10]

Bioenergy can play a critical role for mitigation, but there are issues to consider, such as the sustainability of practices and the efficiency of bioenergy systems (robust evidence, medium agreement) [11.4.4, Box 11.5, 11.13.6, 11.13.7]. Barriers to largescale deployment of bioenergy include concerns about GHG emissions from land, food security, water resources, biodiversity conservation and livelihoods. The scientific debate about the overall climate impact related to land-use competition effects of specific bioenergy pathways remains unresolved (robust evidence, high agreement). [11.4.4, 11.13] Bioenergy technologies are diverse and span a wide range of options and technology pathways. Evidence suggests that options with low lifecycle emissions (e.g., sugar cane, Miscanthus, fast growing tree species, and sustainable use of biomass residues), some already available, can reduce GHG emissions; outcomes are site-specific and rely on efficient integrated 'biomass-to-bioenergy systems', and sustainable land-use management and governance. In some regions, specific bioenergy options, such as improved cookstoves, and small-scale biogas and biopower production, could reduce GHG emissions and improve livelihoods and health in the context of sustainable development (medium evidence, medium agreement). [11.13]

17 UN Programme on Reducing Emissions from Deforestation and Forest Degradation in developing countries, including conservation, sustainable management of forests and enhancement of forest carbon stocks. 


\section{TS.3.2.7 Human settlements, infrastructure, and spatial planning}

Urbanization is a global trend transforming human settlements, societies, and energy use (robust evidence, high agreement). In 1900 , when the global population was 1.6 billion, only $13 \%$ of the population, or some 200 million, lived in urban areas. As of 2011, more than $52 \%$ of the world's population-roughly 3.6 billion—lives in urban areas. By 2050, the urban population is expected to increase to $5.6-7.1$ billion, or $64-69 \%$ of the world population. [12.2]

Urban areas account for more than half of global primary energy use and energy-related $\mathrm{CO}_{2}$ emissions (medium evidence, high agreement). The exact share of urban energy and GHG emissions varies with emission accounting frameworks and definitions. Taking account of direct and indirect emissions, urban areas account for $67-76 \%$ of global energy use (central estimate) and $71-76 \%$ of global energyrelated $\mathrm{CO}_{2}$ emissions. Taking account of direct emissions only, the urban share of emissions is $44 \%$ (Figure TS.32). [12.2, 12.3]

No single factor explains variations in per-capita emissions across cities, and there are significant differences in per capita GHG emissions between cities within a single country (robust evidence, high agreement). Urban GHG emissions are influenced by a variety of physical, economic and social factors, development levels, and urbanization histories specific to each city. Key influences on urban GHG emissions include income, population dynamics, urban form, locational factors, economic structure, and market failures. Per capita final energy use and $\mathrm{CO}_{2}$ emissions in cities of Annex I countries tend to be lower than national averages, in cities of non-Annex I countries they tend to be higher. [12.3]

The majority of infrastructure and urban areas have yet to be built (limited evidence, high agreement). Accounting for trends in declining population densities, and continued economic and population growth, urban land cover is projected to expand by $56-310 \%$ between 2000 and 2030. If the global population increases to 9.3 billion by 2050 and developing countries expand their built environment and infrastructure to current global average levels using available technology of today, the production of infrastructure materials alone would generate about $470 \mathrm{GtCO}_{2}$ emissions. Currently, average per capita $\mathrm{CO}_{2}$ emissions embodied in the infrastructure of industrialized countries is five times larger than those in developing countries. [12.2, 12.3]

Infrastructure and urban form are strongly interlinked, and lock in patterns of land use, transport choice, housing, and behaviour (medium evidence, high agreement). Urban form and infrastructure shape long-term land-use management, influence individual transport choice, housing, and behaviour, and affect the system-wide efficiency of a city. Once in place, urban form and infrastructure are difficult to change (Figure TS.33). [12.2, 12.3, 12.4]
Mitigation options in urban areas vary by urbanization trajectories and are expected to be most effective when policy instruments are bundled (robust evidence, high agreement). For rapidly developing cities, options include shaping their urbanization and infrastructure development towards more sustainable and low-carbon pathways. In mature or established cities, options are constrained by existing urban forms and infrastructure and the potential for refurbishing existing systems and infrastructures. Key mitigation strategies include co-locating high residential with high employment densities,

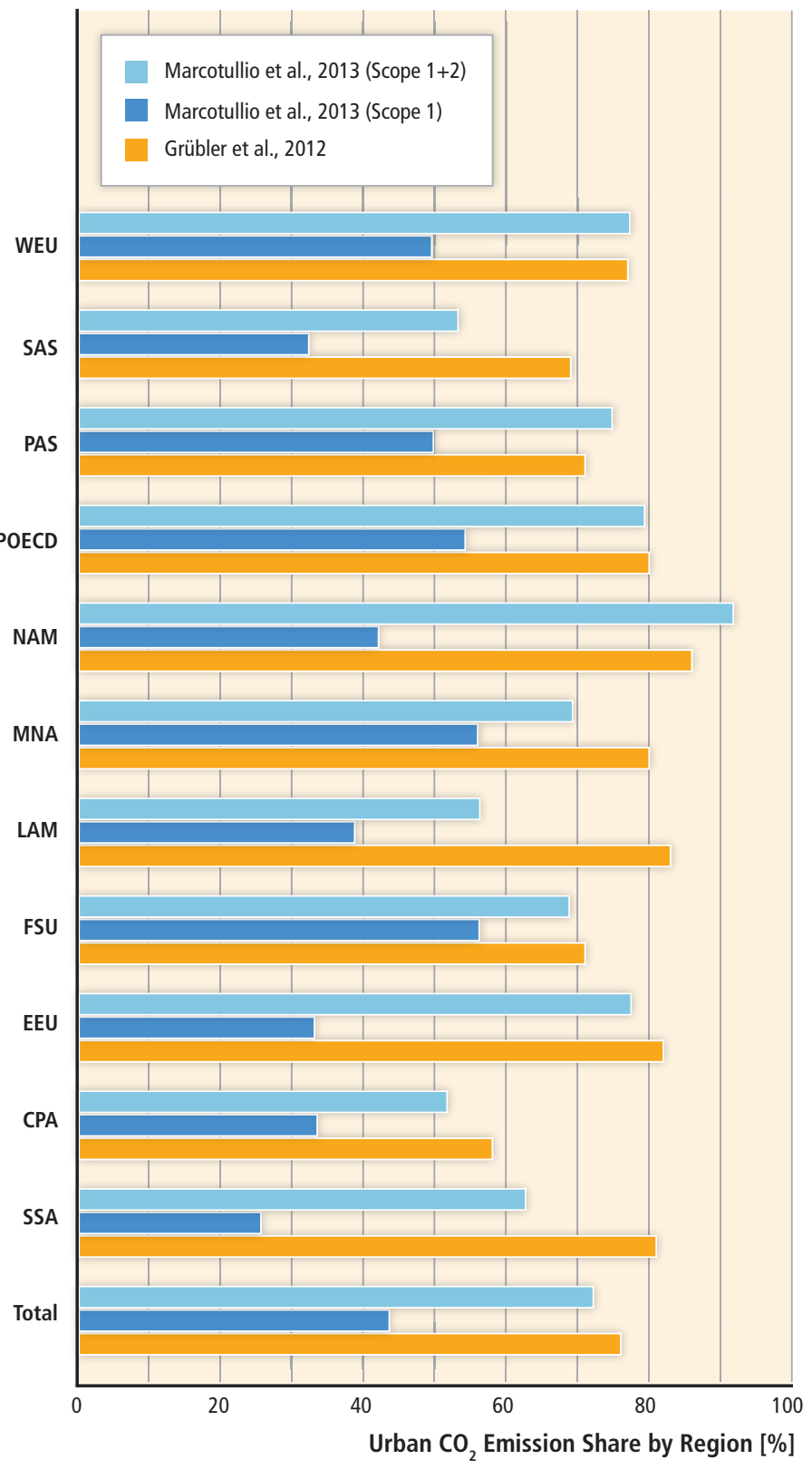

Figure TS.32 Estimated shares of direct (Scope 1) and indirect urban $\mathrm{CO}_{2}$ emissions in total emissions across world regions $\left(\mathrm{GtCO}_{2}\right)$. Indirect emissions (Scope 2) allocate emissions from thermal power plants to urban areas. CPA: Centrally Planned Asia and China; EEU: Central and Eastern Europe; FSU: Former Soviet Union; LAM: Latin America and Caribbean; MNA: Middle East and North Africa; NAM: North America; PAS: South-East Asia and Pacific; POECD: Pacific OECD; SAS: South Asia; SSA: Sub Saharan Africa; WEU: Western Europe. [12.2.2, Figure 12.4] 


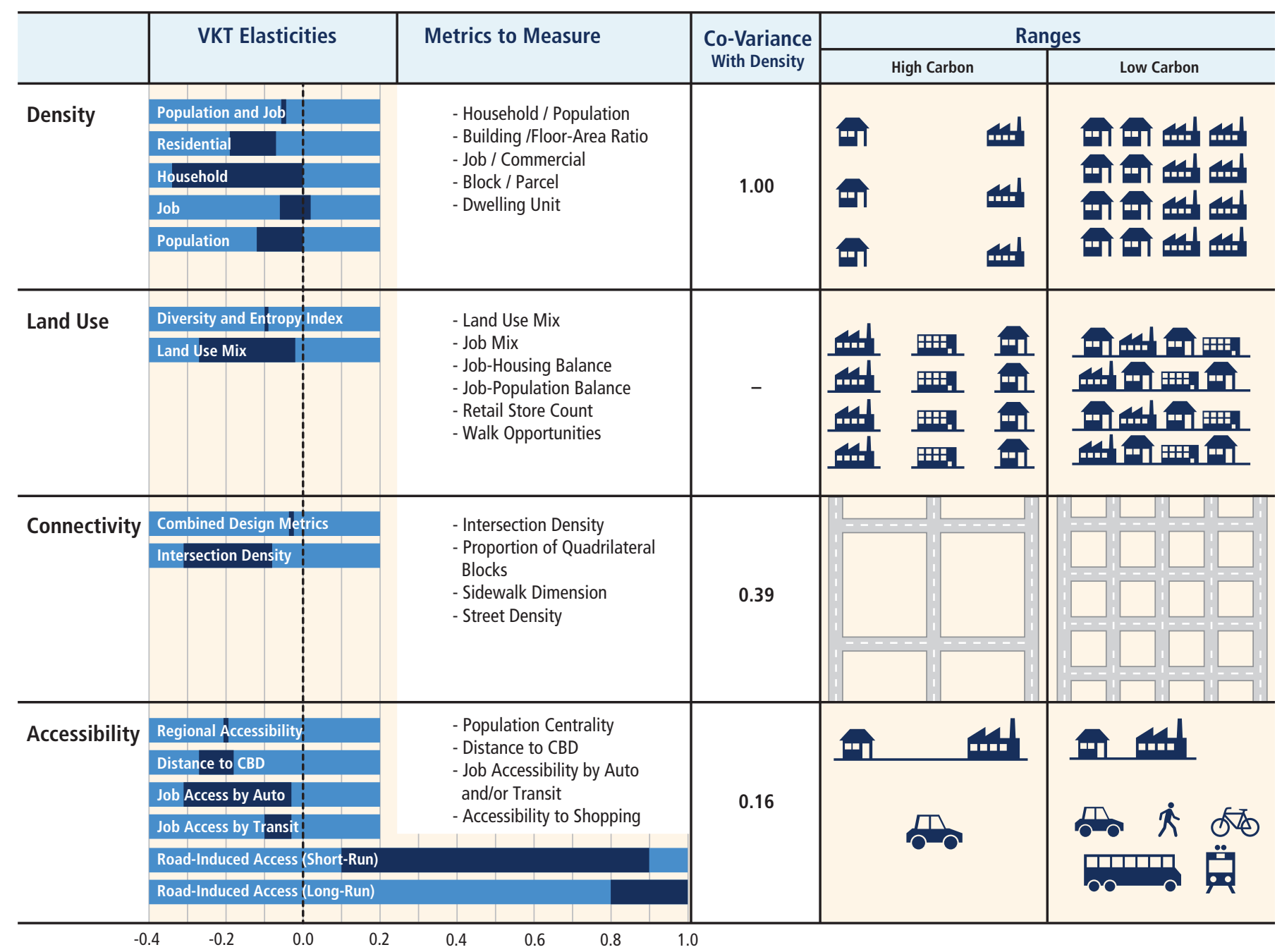

Figure TS.33| Four key aspects of urban form and structure (density, land-use mix, connectivity, and accessibility), their vehicle kilometers travelled (VKT) elasticities, commonly used metrics, and stylized graphics. The dark blue row segments under the VKT elasticities column provide the range of elasticities for the studies included. CBD: Central business district. [Figure 12.14]

achieving high diversity and integration of land uses, increasing accessibility and investing in public transit and other supportive demandmanagement measures (Figure TS.33). Bundling these strategies can reduce emissions in the short term and generate even higher emissions savings in the long term. $[12.4,12.5]$

The largest opportunities for future urban GHG emissions reduction might be in rapidly urbanizing countries where urban form and infrastructure are not locked-in but where there are often limited governance, technical, financial, and institutional capacities (robust evidence, high agreement). The bulk of future infrastructure and urban growth is expected in small- to medium-size cities in developing countries, where these capacities can be limited or weak. $[12.4,12.5,12.6,12.7]$

Thousands of cities are undertaking climate action plans, but their aggregate impact on urban emissions is uncertain (robust evidence, high agreement). Local governments and institutions possess unique opportunities to engage in urban mitigation activities and local mitigation efforts have expanded rapidly. However, little systematic assessment exists regarding the overall extent to which cities are implementing mitigation policies and emissions reduction targets are being achieved, or emissions reduced. Climate action plans include a range of measures across sectors, largely focused on energy efficiency rather than broader land-use planning strategies and cross-sectoral measures to reduce sprawl and promote transit-oriented development (Figure TS.34). [12.6, 12.7, 12.9]

The feasibility of spatial planning instruments for climate change mitigation is highly dependent on a city's financial and governance capability (robust evidence, high agreement). Drivers of urban GHG emissions are interrelated and can be addressed by a number of regulatory, management, and market-based instruments. Many of these instruments are applicable to cities in both developed and developing countries, but the degree to which they can be implemented varies. In addition, each instrument varies in its potential to generate public revenues or require government expenditures, and the administrative scale at which it can be applied (Figure TS.35). A bun- 


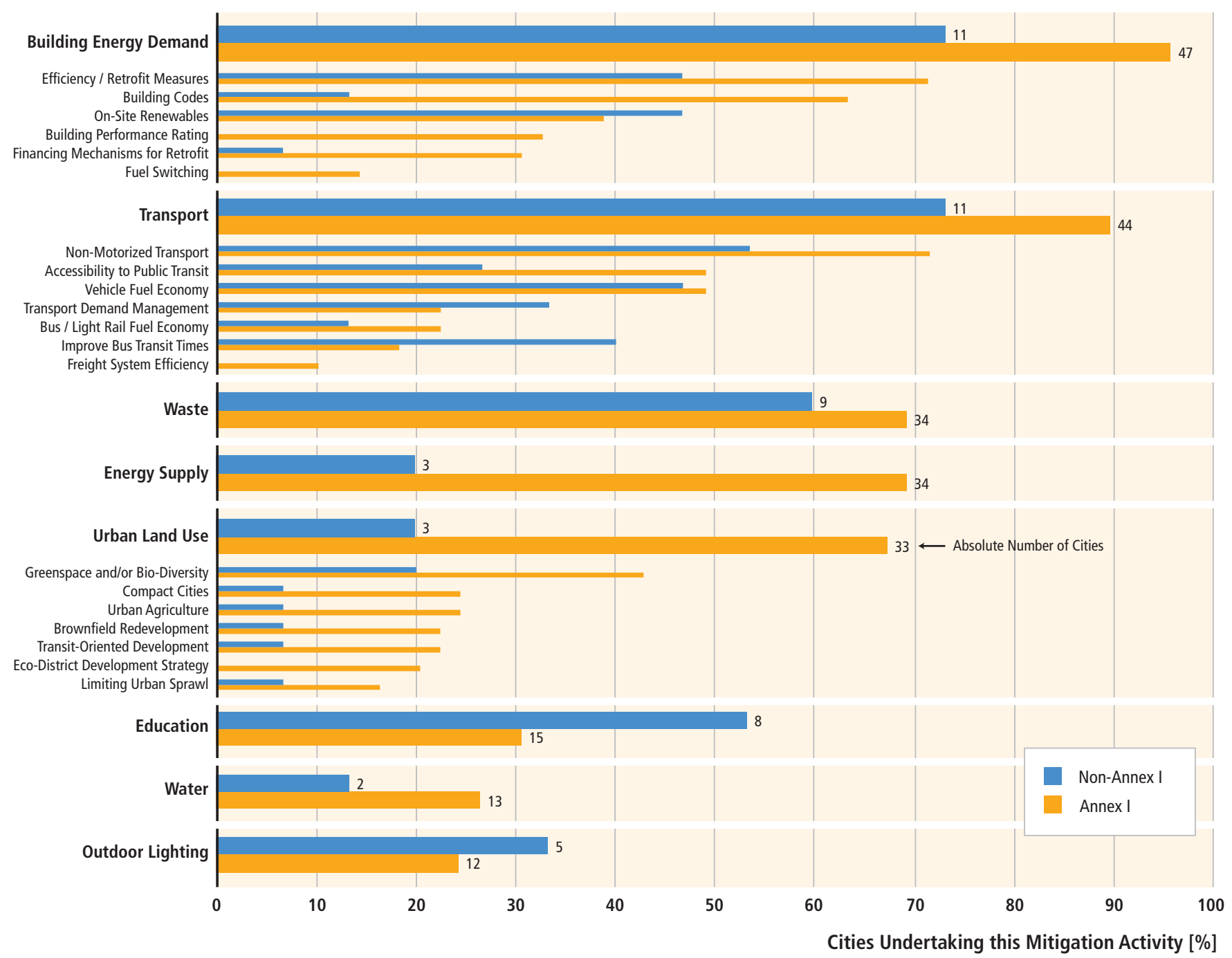

Figure TS.34| Common mitigation measures in Climate Action Plans. [Figure 12.22]

dling of instruments and a high level of coordination across institutions can increase the likelihood of achieving emissions reductions and avoiding unintended outcomes. [12.6, 12.7]

For designing and implementing climate policies effectively, institutional arrangements, governance mechanisms, and financial resources should be aligned with the goals of reducing urban GHG emissions (high confidence). These goals will reflect the specific challenges facing individual cities and local governments. The following have been identified as key factors: (1) institutional arrangements that facilitate the integration of mitigation with other high-priority urban agendas; (2) a multilevel governance context that empowers cities to promote urban transformations; (3) spatial planning competencies and political will to support integrated land-use and transportation planning; and (4) sufficient financial flows and incentives to adequately support mitigation strategies. [12.6, 12.7]

Successful implementation of urban climate change mitigation strategies can provide co-benefits (robust evidence, high agreement). Urban areas throughout the world continue to struggle with challenges, including ensuring access to energy, limiting air and water pollution, and maintaining employment opportunities and competitiveness. Action on urban-scale mitigation often depends on the ability to relate climate change mitigation efforts to local co-benefits. The cobenefits of local climate change mitigation can include public savings, air quality and associated health benefits, and productivity increases in urban centres, providing additional motivation for undertaking mitigation activities. [12.5, 12.6, 12.7, 12.8] 


\section{Government Scale}

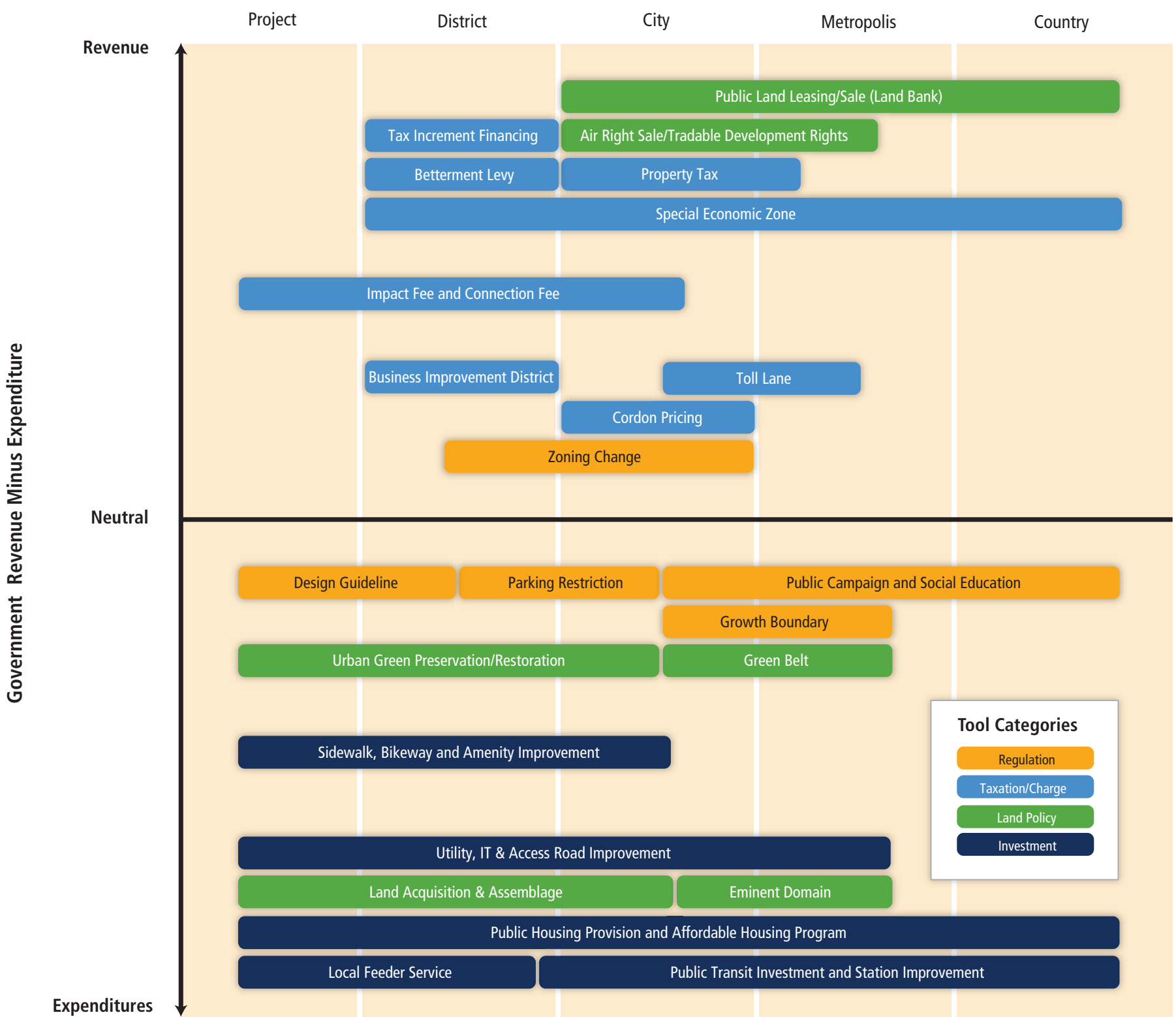

Figure TS.35 | Key spatial planning tools and effects on government revenues and expenditures across administrative scales. Figure shows four key spatial planning tools (coded in colours) and the scale of governance at which they are administered ( $x$-axis) as well as how much public revenue or expenditure the government generates by implementing each instrument (y-axis). [Figure 12.20]

\section{TS.4 Mitigation policies and institutions}

The previous section shows that since AR4 the scholarship on mitigation pathways has begun to consider in much more detail how a variety of real-world considerations-such as institutional and political constraints, uncertainty associated with climate change risks, the availability of technologies and other factors-affect the kinds of policies and measures that are adopted. Those factors have important implications for the design, cost, and effectiveness of mitigation action. This sec- tion focuses on how governments and other actors in the private and public sectors design, implement, and evaluate mitigation policies. It considers the 'normative' scientific research on how policies should be designed to meet particular criteria. It also considers research on how policies are actually designed and implemented a field known as 'positive' analysis. The discussion first characterizes fundamental conceptual issues, and then presents a summary of the main findings from WGIII AR5 on local, national, and sectoral policies. Much of the practical policy effort since AR4 has occurred in these contexts. From there the summary looks at ever-higher levels of aggregation, ultimately ending at the global level and cross-cutting investment and finance issues. 


\section{TS.4.1 Policy design, behaviour and political economy}

There are multiple criteria for evaluating policies. Policies are frequently assessed according to four criteria [3.7.1, 13.2.2, 15.4.1]:

- Environmental effectiveness-whether policies achieve intended goals in reducing emissions or other pressures on the environment or in improving measured environmental quality.

- Economic effectiveness-the impact of policies on the overall economy. This criterion includes the concept of economic efficiency, the principle of maximizing net economic benefits. Economic welfare also includes the concept of cost-effectiveness, the principle of attaining a given level of environmental performance at lowest aggregate cost.

- Distributional and social impacts—also known as 'distributional equity, this criterion concerns the allocation of costs and benefits of policies to different groups and sectors within and across economies over time. It includes, often, a special focus on impacts on the least well-off members of societies within countries and around the world.

- Institutional and political feasibility-whether policies can be implemented in light of available institutional capacity, the political constraints that governments face, and other factors that are essential to making a policy viable.

All criteria can be applied with regard to the immediate 'static' impacts of policies and from a long-run 'dynamic' perspective that accounts for the many adjustments in the economic, social and political systems. Criteria may be mutually reinforcing, but there may also be conflicts or tradeoffs among them. Policies designed for maximum environmental effectiveness or economic performance may fare less well on other criteria, for example. Such tradeoffs arise at multiple levels of governing systems. For example, it may be necessary to design international agreements with flexibility so that it is feasible for a large number of diverse countries to accept them, but excessive flexibility may undermine incentives to invest in cost-effective long-term solutions.

Policymakers make use of many different policy instruments at the same time. Theory can provide some guidance on the normative advantages and disadvantages of alternative policy instruments in light of the criteria discussed above. The range of different policy instruments includes $[3.8,15.3]$ :

- Economic incentives, such as taxes, tradable allowances, fines, and subsidies

- Direct regulatory approaches, such as technology or performance standards

- Information programmes, such as labelling and energy audits

- Government provision, for example of new technologies or in state enterprises

- Voluntary actions, initiated by governments, firms, and non-governmental organizations (NGOs)
Since AR4, the inventory of research on these different instruments has grown, mostly with reference to experiences with policies adopted within particular sectors and countries as well as the many interactions between policies. One implication of that research has been that international agreements that aim to coordinate across countries reflect the practicalities on the particular policy choices of national governments and other jurisdictions.

The diversity in policy goals and instruments highlights differences in how sectors and countries are organized economically and politically as well as the multi-level nature of mitigation. Since AR4, one theme of research in this area has been that the success of mitigation measures depends in part on the presence of institutions capable of designing and implementing regulatory policies and the willingness of respective publics to accept these policies. Many policies have effects, sometimes unanticipated, across multiple jurisdictions-across cities, regions and countries-because the economic effects of policies and the technological options are not contained within a single jurisdiction. [13.2.2.3, $14.1 .3,15.2,15.9]$

Interactions between policy instruments can be welfare-enhancing or welfare-degrading. The chances of welfare-enhancing interactions are particularly high when policy instruments address multiple different market failures-for example, a subsidy or other policy instrument aimed at boosting investment in R\&D on less emission-intensive technologies can complement policies aimed at controlling emissions, as can regulatory intervention to support efficient improvement of enduse energy efficiency. By contrast, welfare-degrading interactions are particularly likely when policies are designed to achieve identical goals. Narrowly targeted policies such as support for deployment (rather than $R \& D$ ) of particular energy technologies that exist in tandem with broader economy-wide policies aimed at reducing emissions (for example, a cap-and-trade emissions scheme) can have the effect of shifting the mitigation effort to particular sectors of the economy in ways that typically result in higher overall costs. [3.8.6, 15.7, 15.8]

There are a growing number of countries devising policies for adaptation, as well as mitigation, and there may be benefits to considering the two within a common policy framework (medium evidence, low agreement). However, there are divergent views on whether adding adaptation to mitigation measures in the policy portfolio encourages or discourages participation in international cooperation [1.4.5, 13.3.3]. It is recognized that an integrated approach can be valuable, as there exist both synergies and tradeoffs [16.6].

Traditionally, policy design, implementation, and evaluation has focused on governments as central designers and implementers of policies, but new studies have emerged on government acting in a coordinating role (medium confidence). In these cases, governments themselves seek to advance voluntary approaches, especially when traditional forms of regulation are thought to be inadequate or 
the best choices of policy instruments and goals is not yet apparent. Examples include voluntary schemes that allow individuals and firms to purchase emission credits that offset the emissions associated with their own activities such as flying and driving. Since AR4, a substantial new literature has emerged to examine these schemes from positive and normative perspectives. [13.12, 15.5.7]

The successful implementation of policy depends on many factors associated with human and institutional behaviour (very high confidence). One of the challenges in designing effective instruments is that the activities that a policy is intended to affect-such as the choice of energy technologies and carriers and a wide array of agricultural and forestry practices-are also influenced by social norms, decision-making rules, behavioural biases, and institutional processes $[2.4,3.10]$. There are examples of policy instruments made more effective by taking these factors into account, such as in the case of financing mechanisms for household investments in energy efficiency and renewable energy that eliminate the need for up-front investment [2.4, 2.6.5.3]. Additionally, the norms that guide acceptable practices could have profound impacts on the baselines against which policy interventions are evaluated, either magnifying or reducing the required level of policy intervention $[1.2 .4,4.3,6.5 .2]$.

Climate policy can encourage investment that may otherwise be suboptimal because of market imperfections (very high con- fidence). Many of the options for energy efficiency as well as lowcarbon energy provision require high up-front investment that is often magnified by high-risk premiums associated with investments in new technologies. The relevant risks include those associated with future market conditions, regulatory actions, public acceptance, and technology cost and performance. Dedicated financial instruments exist to lower these risks for private actors - for example, credit insurance, feed-in tariffs (FITs), concessional finance, or rebates [16.4]. The design of other mitigation policies can also incorporate elements to help reduce risks, such as a cap-and-trade regime that includes price floors and ceilings $[2.6 .5,15.5,15.6]$.

\section{TS.4.2 Sectoral and national policies}

There has been a considerable increase in national and subnational mitigation plans and strategies since AR4 (Figure TS.36). These plans and strategies are in their early stages of development and implementation in many countries, making it difficult to assess whether and how they will result in appropriate institutional and policy change, and therefore, their impact on future GHG emissions. However, to date these policies, taken together, have not yet achieved a substantial deviation in GHG emissions from the past trend. Theories of institutional change suggest they might play a role in shaping incentives, political contexts, and policy paradigms in a way that encourages
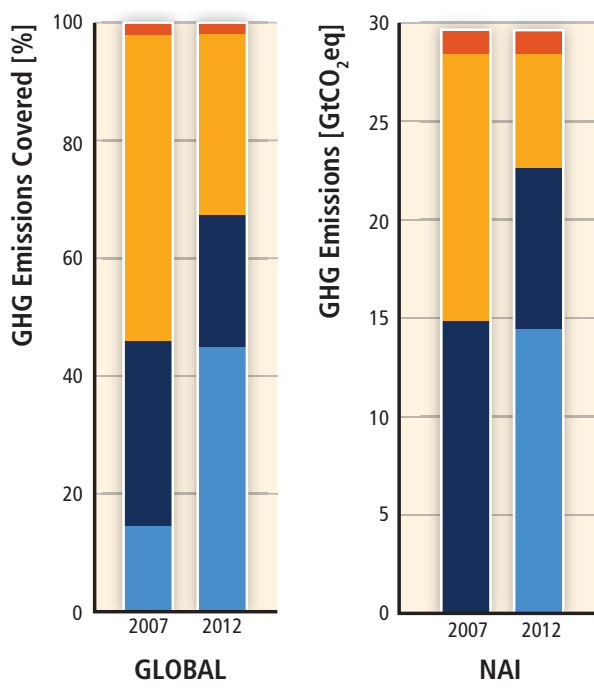

NAI

\begin{tabular}{l|l|l}
\hline 1 & $7(15 \%)$ & $22(44 \%)$ \\
\hline 2 & $15(30 \%)$ & $11(23 \%)$ \\
\hline 3 & $25(52 \%)$ & $15(30 \%)$ \\
\hline 4 & $1(3 \%)$ & $1(3 \%)$ \\
\hline
\end{tabular}

\begin{tabular}{l|l|l}
\hline 1 & $0(0 \%)$ & $14(49 \%)$ \\
\hline 2 & $15(50 \%)$ & $8(28 \%)$ \\
\hline 3 & $14(46 \%)$ & $6(19 \%)$ \\
\hline 4 & $1(4 \%)$ & $1(4 \%)$ \\
\hline
\end{tabular} \begin{tabular}{lll|l}
\hline $0(38 \%)$ & $7(38 \%)$ \\
\hline $12(61 \%)$ & $9(16 \%)$ \\
\hline $0(1 \%)$ & $0(1 \%)$ \\
\hline
\end{tabular}

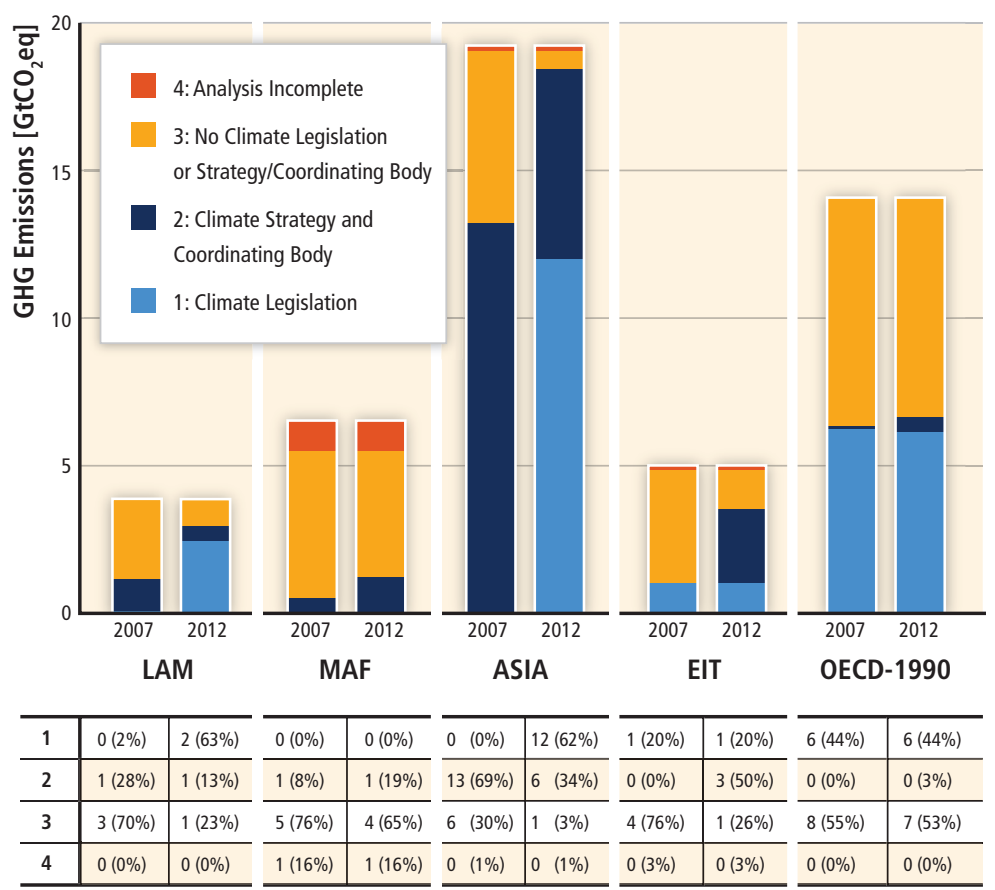

Figure TS.36 National climate legislation and strategies in 2007 and 2012. Regions include NAI (Non Annex I countries—developing countries), Al (Annex I countries—developed countries), LAM (Latin America), MAF (Middle East and Africa), ASIA (Asia), EIT (Economies in Transition), OECD-1990; see Annex II.2 for more details. In this figure, climate legislation is defined as mitigation-focused legislation that goes beyond sectoral action alone. Climate strategy is defined as a non-legislative plan or framework aimed at mitigation that encompasses more than a small number of sectors, and that includes a coordinating body charged with implementation. International pledges are not included, nor are subnational plans and strategies. The panel shows proportion of GHG emissions covered. [Figure 15.1] 
GHG emissions reductions in the future $[15.1,15.2]$. However, many baseline scenarios (i.e., those without additional mitigation policies) show concentrations that exceed 1000 ppm $\mathrm{CO}_{2}$ eq by 2100 , which is far from a concentration with a likely probability of maintaining temperature increases below $2^{\circ} \mathrm{C}$ this century. Mitigation scenarios suggest that a wide range of environmentally effective policies could be enacted that would be consistent with such goals [6.3]. In practice, climate strategies and the policies that result are influenced by political economy factors, sectoral considerations, and the potential for realizing co-benefits. In many countries, mitigation policies have also been actively pursued at state and local levels. $[15.2,15.5,15.8]$

Since AR4, there is growing political and analytical attention to co-benefits and adverse side-effects of climate policy on other objectives and vice versa that has resulted in an increased focus on policies designed to integrate multiple objectives (high confidence). Co-benefits are often explicitly referenced in climate and sectoral plans and strategies and often enable enhanced political support [15.2]. However, the analytical and empirical underpinnings for many of these interactive effects, and particularly for the associated welfare impacts, are under-developed $[1.2,3.6 .3,4.2,4.8,6.6]$. The scope for co-benefits is greater in low-income countries, where complementary policies for other objectives, such as air quality, are often weak [5.7, 6.6, 15.2].

The design of institutions affects the choice and feasibility of policy options as well as the sustainable financing of mitigation measures. Institutions designed to encourage participation by representatives of new industries and technologies can facilitate transitions to low-GHG emissions pathways $[15.2,15.6]$. Policies vary in the extent to which they require new institutional capabilities to be implemented. Carbon taxation, in most settings, can rely mainly on existing tax infrastructure and is administratively easier to implement than many other alternatives such as cap-and-trade systems [15.5]. The extent of institutional innovation required for policies can be a factor in instrument choice, especially in developing countries.

Sector-specific policies have been more widely used than economy-wide, market-based policies (medium evidence, high agreement). Although economic theory suggests that market-based, economywide policies for the singular objective of mitigation would generally be more cost-effective than sector-specific policies, political economy considerations often make economy-wide policies harder to design and implement than sector-specific policies [15.2.3, 15.2.6, 15.5.1]. In some countries, emission trading and taxes have been enacted to address the market externalities associated with GHG emissions, and have contributed to the fulfilment of sector-specific GHG reduction goals (medium evidence, medium agreement) [7.12]. In the longer term, GHG pricing can support the adoption of low-GHG energy technologies. Even if economy-wide policies were implemented, sector-specific policies may be needed to overcome sectoral market failures. For example, building codes can require energy-efficient investments where private investments would otherwise not exist [9.10]. In transport, pricing policies that raise the cost of carbon-intensive forms of private transport are more effective when backed by public investment in viable alternatives [8.10]. Table TS.9 presents a range of sector-specific policies that have been implemented in practice. $[15.1,15.2,15.5,15.8,15.9]$

Carbon taxes have been implemented in some countries and-alongside technology and other policies-have contributed to decoupling of emissions from GDP (high confidence). Differentiation by sector, which is quite common, reduces cost-effectiveness that arises from the changes in production methods, consumption patterns, lifestyle shifts, and technology development, but it may increase political feasibility, or be preferred for reasons of competitiveness or distributional equity. In some countries, high carbon and fuel taxes have been made politically feasible by refunding revenues or by lowering other taxes in an environmental fiscal reform. Mitigation policies that raise government revenue (e.g., auctioned emission allowances under a cap-and-trade system or emission taxes) generally have lower social costs than approaches that do not, but this depends on how the revenue is used [3.6.3]. [15.2, 15.5.2, 15.5.3]

Fuel taxes are an example of a sector-specific policy and are often originally put in place for objectives such as revenue-they are not necessarily designed for the purpose of mitigation (high confidence). In Europe, where fuel taxes are highest, they have contributed to reductions in carbon emissions from the transport sector of roughly $50 \%$ for this group of countries. The short-run response to higher fuel prices is often small, but long-run price elasticities are quite high, or roughly -0.6 to -0.8 . This means that in the long run, $10 \%$ higher fuel prices correlate with $7 \%$ reduction in fuel use and emissions. In the transport sector, taxes have the advantage of being progressive or neutral in most countries and strongly progressive in low-income countries. [15.5.2]

Cap-and-trade systems for GHG emissions are being established in a growing number of countries and regions. Their environmental effect has so far been limited because caps have either been loose or have not yet been binding (limited evidence, medium agreement). There appears to have been a tradeoff between the political feasibility and environmental effectiveness of these programmes, as well as between political feasibility and distributional equity in the allocation of permits. Greater environmental effectiveness through a tighter cap may be combined with a price ceiling that improves political feasibility. $[14.4 .2,15.5 .3]$

Different factors reduced the price of European Union Emissions Trading System (EU ETS) allowances below anticipated levels, thereby slowing investment in mitigation (high confidence). While the European Union demonstrated that a cross-border cap-and-trade system can work, the low price of EU ETS allowances in recent years provided insufficient incentives for significant additional investment in mitigation. The low price is related to unexpected depth and duration of the economic recession, uncertainty about the long-term reduction targets for GHG emissions, import of credits from the Clean Development Mechanism (CDM), and the interaction with other policy instruments, 
Table TS.9 | Sector policy instruments. The table brings together evidence on mitigation policy instruments discussed in Chapters 7 to 12. [Table 15.2]

\begin{tabular}{|c|c|c|c|c|c|c|}
\hline Policy Instruments & Energy [7.12] & Transport [8.10] & Buildings [9.10] & Industry [10.11] & AFOLU [11.10] & $\begin{array}{l}\text { Human Settlements } \\
\text { and Infrastructure }\end{array}$ \\
\hline $\begin{array}{l}\text { Economic Instru- } \\
\text { ments-Taxes } \\
\text { (Carbon taxes may } \\
\text { be economy-wide) }\end{array}$ & - Carbon taxes & $\begin{array}{l}\text { - Fuel taxes } \\
\text { - Congestion charges, } \\
\text { vehicle registration } \\
\text { fees, road tolls } \\
\text { - Vehicle taxes }\end{array}$ & $\begin{array}{l}\text { - Carbon and/or energy } \\
\text { taxes (either sectoral } \\
\text { or economy wide) }\end{array}$ & $\begin{array}{l}\text { - Carbon tax or } \\
\text { energy tax } \\
\text { - Waste disposal } \\
\text { taxes or charges }\end{array}$ & $\begin{array}{l}\text { - Fertilizer or Nitrogen } \\
\text { taxes to reduce } \\
\text { nitrous oxide }\end{array}$ & $\begin{array}{l}\text { - Sprawl taxes, Impact } \\
\text { fees, exactions, split- } \\
\text { rate property taxes, } \\
\text { tax increment finance, } \\
\text { betterment taxes, } \\
\text { congestion charges }\end{array}$ \\
\hline $\begin{array}{l}\text { Economic Instru- } \\
\text { ments-Tradable } \\
\text { Allowances } \\
\text { (May be econ- } \\
\text { omy-wide) }\end{array}$ & $\begin{array}{l}\text { - Emissions trading } \\
\text { (e.g., EU ETS) } \\
\text { - Emission credits } \\
\text { under CDM } \\
\text { - Tradable Green } \\
\text { Certificates }\end{array}$ & $\begin{array}{l}\text { - Fuel and vehicle } \\
\text { standards }\end{array}$ & $\begin{array}{l}\text { - Tradable certificates } \\
\text { for energy efficiency } \\
\text { improvements } \\
\text { (white certificates) }\end{array}$ & $\begin{array}{l}\text { - Emissions trading } \\
\text { - Emission credit } \\
\text { under CDM } \\
\text { - Tradable Green } \\
\text { Certificates }\end{array}$ & $\begin{array}{l}\text { - Emission credits under } \\
\text { the Kyoto Protocol's } \\
\text { Clean Development } \\
\text { Mechanism (CDM) } \\
\text { - Compliance schemes } \\
\text { outside Kyoto protocol } \\
\text { (national schemes) } \\
\text { - Voluntary carbon } \\
\text { markets }\end{array}$ & $\begin{array}{l}\text { - Urban-scale Cap } \\
\text { and Trade }\end{array}$ \\
\hline $\begin{array}{l}\text { Economic Instru- } \\
\text { ments-Subsidies }\end{array}$ & $\begin{array}{l}\text { - Fossil fuel subsidy } \\
\text { removal } \\
\text { - Feed-in-tariffs for } \\
\text { renewable energy } \\
\text { - Capital subsidies } \\
\text { and insurance for 1st } \\
\text { generation Carbon } \\
\text { Dioxide Capture } \\
\text { and Storage (CCS) }\end{array}$ & $\begin{array}{l}\text { - Biofuel subsidies } \\
\text { - Vehicle purchase } \\
\text { subsidies } \\
\text { - Feebates }\end{array}$ & $\begin{array}{l}\text { - Subsidies or Tax } \\
\text { exemptions for } \\
\text { investment in efficient } \\
\text { buildings, retrofits } \\
\text { and products } \\
\text { - Subsidized loans }\end{array}$ & $\begin{array}{l}\text { - Subsidies (e.g., for } \\
\text { energy audits) } \\
\text { - Fiscal incentives (e.g., } \\
\text { for fuel switching) }\end{array}$ & $\begin{array}{l}\text { - Credit lines for low } \\
\text { carbon agriculture, } \\
\text { sustainable forestry. }\end{array}$ & $\begin{array}{l}\text { - Special Improvement } \\
\text { or Redevelopment } \\
\text { Districts }\end{array}$ \\
\hline $\begin{array}{l}\text { Regulatory } \\
\text { Approaches }\end{array}$ & $\begin{array}{l}\text { - Efficiency or } \\
\text { environmental } \\
\text { performance standards } \\
\text { - Renewable Portfolio } \\
\text { standards for } \\
\text { renewable energy } \\
\text { - Equitable access } \\
\text { to electricity grid } \\
\text { - Legal status of long } \\
\text { term } \mathrm{CO}_{2} \text { storage }\end{array}$ & $\begin{array}{l}\text { - Fuel economy } \\
\text { performance standards } \\
\text { - Fuel quality standards } \\
\text { - GHG emission } \\
\text { performance standards } \\
\text { - Regulatory restrictions } \\
\text { to encourage modal } \\
\text { shifts (road to rail) } \\
\text { - Restriction on } \\
\text { use of vehicles in } \\
\text { certain areas } \\
\text { - Environmental capacity } \\
\text { constraints on airports } \\
\text { - Urban planning and } \\
\text { zoning restrictions }\end{array}$ & $\begin{array}{l}\text { - Building codes } \\
\text { and standards } \\
\text { - Equipment and } \\
\text { appliance standards } \\
\text { - Mandates for energy } \\
\text { retailers to assist } \\
\text { customers invest in } \\
\text { energy efficiency }\end{array}$ & $\begin{array}{l}\text { - Energy efficiency } \\
\text { standards for } \\
\text { equipment } \\
\text { - Energy management } \\
\text { systems (also } \\
\text { voluntary) } \\
\text { - Voluntary agreements } \\
\text { (where bound } \\
\text { by regulation) } \\
\text { - Labelling and } \\
\text { public procurement } \\
\text { regulations }\end{array}$ & $\begin{array}{l}\text { - National policies } \\
\text { to support REDD+ } \\
\text { including monitoring, } \\
\text { reporting and } \\
\text { verification } \\
\text { - Forest law to reduce } \\
\text { deforestation } \\
\text { - Air and water pollution } \\
\text { control GHG precursors } \\
\text { - Land-use planning } \\
\text { and governance }\end{array}$ & $\begin{array}{l}\text { - Mixed use zoning } \\
\text { - Development } \\
\text { restrictions } \\
\text { - Affordable housing } \\
\text { mandates } \\
\text { - Site access controls } \\
\text { - Transfer development } \\
\text { rights } \\
\text { - Design codes } \\
\text { - Building codes } \\
\text { - Street codes } \\
\text { - Design standards }\end{array}$ \\
\hline $\begin{array}{l}\text { Information } \\
\text { Programmes }\end{array}$ & & $\begin{array}{l}\text { - Fuel labelling } \\
\text { - Vehicle efficiency } \\
\text { labelling }\end{array}$ & $\begin{array}{l}\text { - Energy audits } \\
\text { - Labelling programmes } \\
\text { - Energy advice } \\
\text { programmes }\end{array}$ & $\begin{array}{l}\text { - Energy audits } \\
\text { - Benchmarking } \\
\text { - Brokerage for } \\
\text { industrial cooperation }\end{array}$ & $\begin{array}{l}\text { - Certification schemes } \\
\text { for sustainable } \\
\text { forest practices } \\
\text { - Information policies } \\
\text { to support REDD+ } \\
\text { including monitoring, } \\
\text { reporting and } \\
\text { verification }\end{array}$ & \\
\hline $\begin{array}{l}\text { Government } \\
\text { Provision of Public } \\
\text { Goods or Services }\end{array}$ & $\begin{array}{l}\text { - Research and } \\
\text { development } \\
\text { - Infrastructure } \\
\text { expansion (district } \\
\text { heating/cooling or } \\
\text { common carrier) }\end{array}$ & $\begin{array}{l}\text { - Investment in } \\
\text { transit and human } \\
\text { powered transport } \\
\text { - Investment in } \\
\text { alternative fuel } \\
\text { infrastructure } \\
\text { - Low emission vehicle } \\
\text { procurement }\end{array}$ & $\begin{array}{l}\text { - Public procurement } \\
\text { of efficient buildings } \\
\text { and appliances }\end{array}$ & $\begin{array}{l}\text { - Training and education } \\
\text { - Brokerage for } \\
\text { industrial cooperation }\end{array}$ & $\begin{array}{l}\text { - Protection of national, } \\
\text { state, and local forests. } \\
\text { - Investment in } \\
\text { improvement and } \\
\text { diffusion of innovative } \\
\text { technologies in } \\
\text { agriculture and forestry }\end{array}$ & $\begin{array}{l}\text { - Provision of utility } \\
\text { infrastructure such as } \\
\text { electricity distribution, } \\
\text { district heating/cooling } \\
\text { and wastewater } \\
\text { connections, etc. } \\
\text { - Park improvements } \\
\text { - Trail improvements } \\
\text { - Urban rail }\end{array}$ \\
\hline Voluntary Actions & & & $\begin{array}{l}\text { - Labelling programmes } \\
\text { for efficient buildings } \\
\text { - Product eco-labelling }\end{array}$ & $\begin{array}{l}\text { - Voluntary agreements } \\
\text { on energy targets or } \\
\text { adoption of energy } \\
\text { management systems, } \\
\text { or resource efficiency }\end{array}$ & $\begin{array}{l}\text { - Promotion of } \\
\text { sustainability by } \\
\text { developing standards } \\
\text { and educational } \\
\text { campaigns }\end{array}$ & \\
\hline
\end{tabular}


particularly related to the expansion of renewable energy as well as regulation on energy efficiency. It has proven to be politically difficult to address this problem by removing GHG emission permits temporarily, tightening the cap, or providing a long-term mitigation goal. [14.4.2]

Adding a mitigation policy to another may not necessarily enhance mitigation. For instance, if a cap-and-trade system has a sufficiently stringent cap then other policies such as renewable subsidies have no further impact on total GHG emissions (although they may affect costs and possibly the viability of more stringent future targets). If the cap is loose relative to other policies, it becomes ineffective. This is an example of a negative interaction between policy instruments. Since other policies cannot be 'added on' to a cap-and-trade system, if it is to meet any particular target, a sufficiently low cap is necessary. A carbon tax, on the other hand, can have an additive environmental effect to policies such as subsidies to renewables. [15.7]

Reduction of subsidies to fossil energy can achieve significant emission reductions at negative social cost (very high confidence). Although political economy barriers are substantial, many countries have reformed their tax and budget systems to reduce fuel subsidies that actually accrue to the relatively wealthy, and utilized lump-sum cash transfers or other mechanisms that are more targeted to the poor. [15.5.3]

Direct regulatory approaches and information measures are widely used, and are often environmentally effective, though debate remains on the extent of their environmental impacts and cost-effectiveness (medium confidence). Examples of regulatory approaches include energy efficiency standards; examples of information programmes include labelling programmes that can help consumers make better-informed decisions. While such approaches often work at a net social benefit, the scientific literature is divided on whether such policies are implemented with negative private costs (see Box TS.12) to firms and individuals $[3.9 .3,15.5 .5,15.5 .6]$. Since AR4 there has been continued investigation into the 'rebound' effects (see Box TS.13) that arise when higher efficiency leads to lower energy costs and greater consumption. There is general agreement that such rebound effects exist, but there is low agreement in the literature on the magnitude $[3.9 .5,5.7 .2,15.5 .4]$.

There is a distinct role for technology policy as a complement to other mitigation policies (high confidence). Properly implemented technology policies reduce the cost of achieving a given environmental target. Technology policy will be most effective when technology-push policies (e.g., publicly funded R\&D) and demand-pull policies (e.g., governmental procurement programmes or performance regulations) are used in a complementary fashion. While technology-push and demand-pull policies are necessary, they are unlikely to be sufficient without complementary framework conditions. Managing social challenges of technology policy change may require innovations in policy and institutional design, including building integrated policies that make complementary use of market incentives, authority, and norms (medium confidence). Since AR4, a large number of countries and subnational jurisdictions have introduced support policies for renewable

\section{Box TS.13 | The rebound effect can reduce energy savings from technological improvement}

Technological improvements in energy efficiency (EE) have direct effects on energy consumption and thus GHG emissions, but can cause other changes in consumption, production, and prices that will, in turn, affect GHG emissions. These changes are generally called 'rebound' or 'takeback' because in most cases they reduce the net energy or emissions reduction associated with the efficiency improvement. The size of EE rebound is controversial, with some research papers suggesting little or no rebound and others concluding that it offsets most or all reductions from EE policies [3.9.5, 5.7.2].

Total EE rebound can be broken down into three distinct parts: substitution-effect, income-effect, and economy-wide effect [3.9.5]. In end-use consumption, substitution-effect rebound, or 'direct rebound' assumes that a consumer will make more use of a device if it becomes more energy efficient because it will be cheaper to use. Income-effect rebound or 'indirect rebound', arises if the improvement in EE makes the consumer wealthier and leads her to consume additional products that require energy. Economywide rebound refers to impacts beyond the behaviour of the entity benefiting directly from the EE improvement, such as the impact of $\mathrm{EE}$ on the price of energy.

Analogous rebound effects for EE improvements in production are substitution towards an input with improved energy efficiency, and substitution among products by consumers when an $\mathrm{EE}$ improvement changes the relative prices of goods, as well as an income effect when an EE improvement lowers production costs and creates greater wealth.

Rebound is sometimes confused with the concept of carbon leakage, which often describes the incentive for emissions-intensive economic activity to migrate away from a region that restricts GHGs (or other pollutants) towards areas with fewer or no restrictions on such emissions $[5.4 .1,14.4]$. Energy efficiency rebound can occur regardless of the geographic scope of the adopted policy. As with leakage, however, the potential for significant rebound illustrates the importance of considering the full equilibrium effects of a mitigation policy $[3.9 .5,15.5 .4]$. 
energy such as feed-in tariffs and renewable portfolio standards. These have promoted substantial diffusion and innovation of new energy technologies such as wind turbines and photovoltaic panels, but have raised questions about their economic efficiency, and introduced challenges for grid and market integration. $[2.6 .5,7.12,15.6 .5]$

Worldwide investment in research in support of mitigation is small relative to overall public research spending (medium confidence). The effectiveness of research support will be greatest if it is increased slowly and steadily rather than dramatically or erratically. It is important that data collection for program evaluation is built into technology policy programmes, because there is limited empirical evidence on the relative effectiveness of different mechanisms for supporting the invention, innovation and diffusion of new technologies. [15.6.2, 15.6.5]

Government planning and provision can facilitate shifts to less energy- and GHG-intensive infrastructure and lifestyles (high confidence). This applies particularly when there are indivisibilities in the provision of infrastructure as in the energy sector [7.6] (e.g., for electricity transmission and distribution or district heating networks); in the transport sector [8.4] (e.g., for non-motorized or public transport); and in urban planning [12.5]. The provision of adequate infrastructure is important for behavioural change [15.5.6].

Successful voluntary agreements on mitigation between governments and industries are characterized by a strong institutional framework with capable industrial associations (medium confidence). The strengths of voluntary agreements are speed and flexibility in phasing measures, and facilitation of barrier removal activities for energy efficiency and low-emission technologies. Regulatory threats, even though the threats are not always explicit, are also an important factor for firms to be motivated. There are few environmental impacts without a proper institutional framework. [15.5.7]

\section{TS.4.3 Development and regional cooperation}

Regional cooperation offers substantial opportunities for mitigation due to geographic proximity, shared infrastructure and policy frameworks, trade, and cross-border investment that would be difficult for countries to implement in isolation (high confidence). Examples of possible regional cooperation policies include regionallylinked development of renewable energy power pools, networks of natural gas supply infrastructure, and coordinated policies on forestry. [14.1]

At the same time, there is a mismatch between opportunities and capacities to undertake mitigation (medium confidence). The regions with the greatest potential to leapfrog to low-carbon development trajectories are the poorest developing regions where there are few lock-in effects in terms of modern energy systems and urbanization patterns. However, these regions also have the lowest financial, technological, and institutional capacities to embark on such low-carbon development paths (Figure TS.37) and their cost of waiting is high due to unmet energy and development needs. Emerging economies already have more lock-in effects but their rapid build-up of modern energy systems and urban settlements still offers substantial opportunities for low-carbon development. Their capacity to reorient themselves to low-carbon development strategies is higher, but also faces constraints in terms of finance, technology, and the high cost of delaying the installation of new energy capacity. Lastly, industrialized economies have the largest lock-in effects, but the highest capacities to reorient their energy, transport, and urbanizations systems towards low-carbon development. [14.1.3, 14.3.2]

Regional cooperation has, to date, only had a limited (positive) impact on mitigation (medium evidence, high agreement). Nonetheless, regional cooperation could play an enhanced role in promoting mitigation in the future, particularly if it explicitly incorporates mitigation objectives in trade, infrastructure and energy policies and promotes direct mitigation action at the regional level. [14.4.2, 14.5]

Most literature suggests that climate-specific regional cooperation agreements in areas of policy have not played an important role in addressing mitigation challenges to date (medium confidence). This is largely related to the low level of regional integration and associated willingness to transfer sovereignty to supra-national regional bodies to enforce binding agreements on mitigation. [14.4.2, 14.4.3]

Climate-specific regional cooperation using binding regulationbased approaches in areas of deep integration, such as EU directives on energy efficiency, renewable energy, and biofuels, have had some impact on mitigation objectives (medium confidence). Nonetheless, theoretical models and past experience suggest that there is substantial potential to increase the role of climate-specific regional cooperation agreements and associated instruments, including economic instruments and regulatory instruments. In this context it is important to consider carbon leakage of such regional initiatives and ways to address it. [14.4.2, 14.4.1]

In addition, non-climate-related modes of regional cooperation could have significant implications for mitigation, even if mitigation objectives are not a component (medium confidence). Regional cooperation with non-climate-related objectives but possible mitigation implications, such as trade agreements, cooperation on technology, and cooperation on infrastructure and energy, has to date also had negligible impacts on mitigation. Modest impacts have been found on the level of GHG emissions of members of regional preferential trade areas if these agreements are accompanied with environmental agreements. Creating synergies between adaptation and mitigation can increase the cost-effectiveness of climate change actions. Linking electricity and gas grids at the regional level has also had a modest impact on mitigation as it facilitated greater use of low-carbon and renewable technologies; there is substantial further mitigation potential in such arrangements. [14.4.2] 
a)

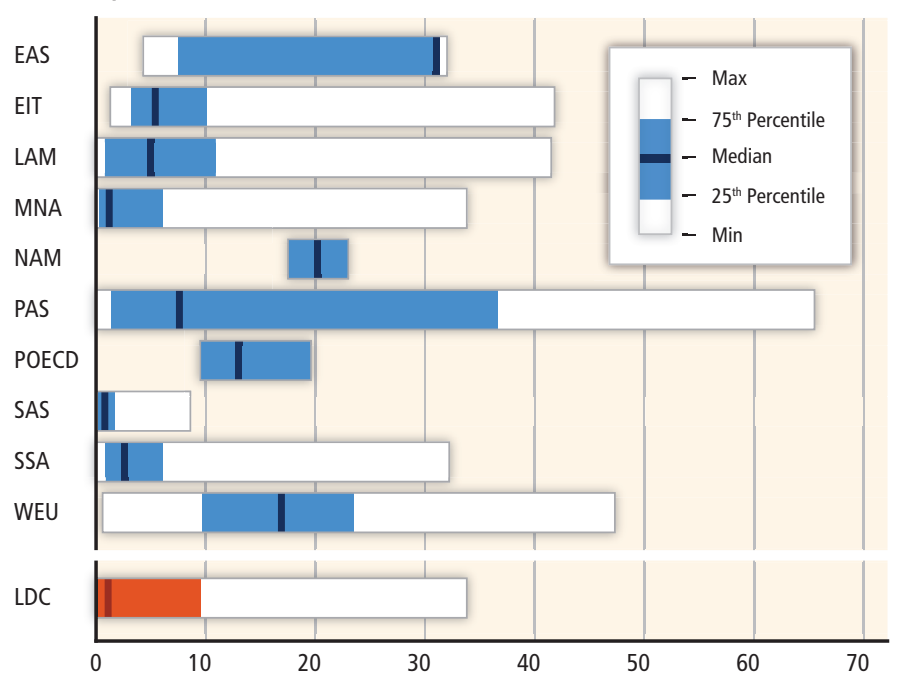

High-Technology Exports [\% of Manufactured Exports]

c)

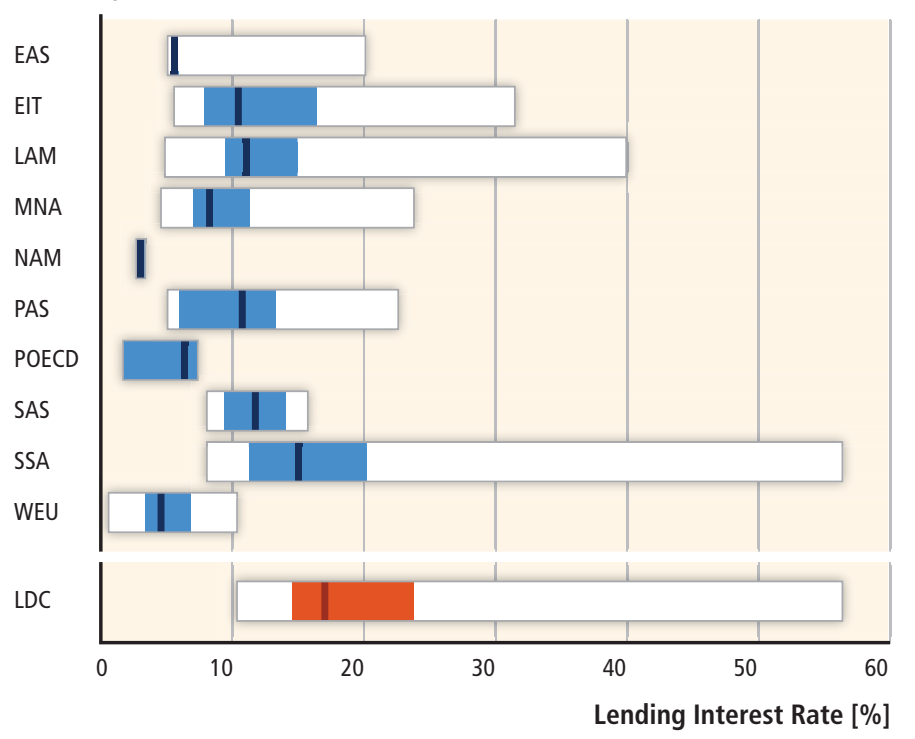

b)

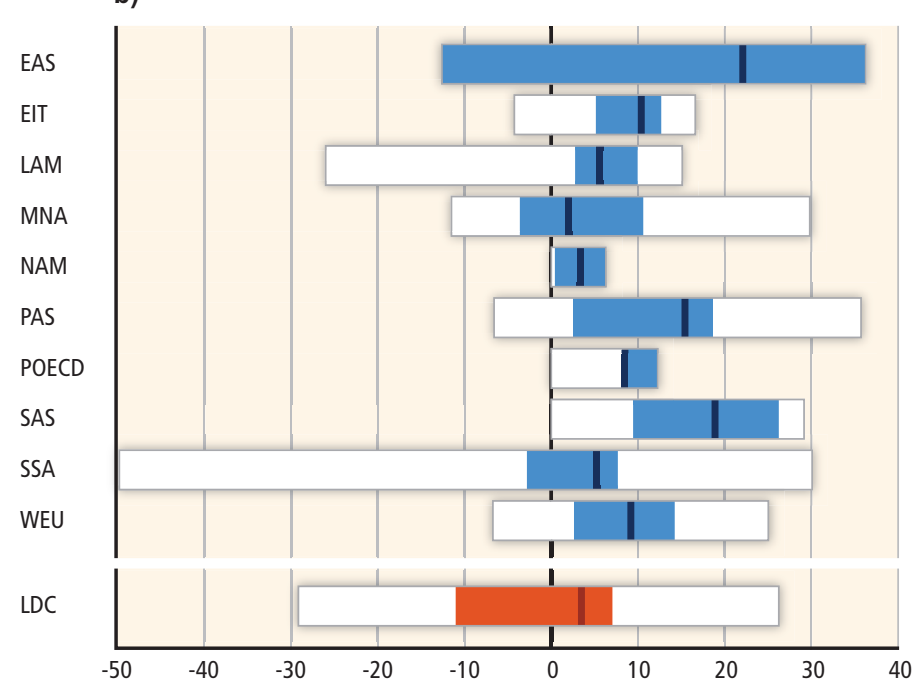

Adjusted Net Savings, Including Particulate Emission Damage [\% of GNI]

d)

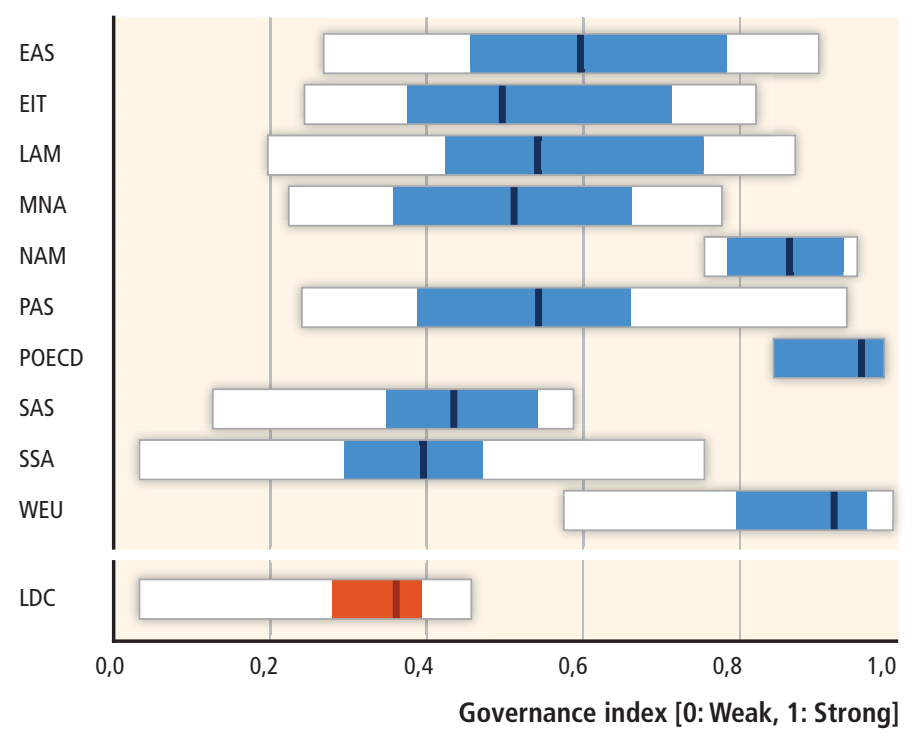

Figure TS.37| Economic and governance indicators affecting regional capacities to embrace mitigation policies. Regions include EAS (East Asia), EIT (Economies in Transition), LAM (Latin America and Caribbean), MNA (Middle East and North Africa), NAM (North America), POECD (Pacific Organisation for Economic Co-operation and Development (OECD)-1990 members), PAS (South East Asia and Pacific), SAS (South Asia), SSA (sub-Saharan Africa), WEU (Western Europe), LDC (least-developed countries). Statistics refer to the year 2010 or the most recent year available. Note: The lending interest rate refers to the average interest rate charged by banks to private sector clients for short- to medium-term financing needs. The governance index is a composite measure of governance indicators compiled from various sources, rescaled to a scale of 0 to 1 , with 0 representing weakest governance and 1 representing strongest governance. [Figure 14.2]

\section{TS.4.4 International cooperation}

Climate change mitigation is a global commons problem that requires international cooperation, but since AR4, scholarship has emerged that emphasizes a more complex and multi-faceted view of climate policy (very high confidence). Two characteristics of climate change necessitate international cooperation: climate change is a global commons problem, and it is characterized by a high degree of heterogeneity in the origins of GHG emissions, mitigation opportunities, climate impacts, and capacity for mitigation and adapta- tion [13.2.1.1]. Policymaking efforts to date have primarily focused on international cooperation as a task centrally focused on the coordination of national policies that would be adopted with the goal of mitigation. More recent policy developments suggest that there is a more complicated set of relationships between national, regional, and global policymaking, based on a multiplicity of goals, a recognition of policy co-benefits, and barriers to technological innovation and diffusion [1.2, $6.6,15.2]$. A major challenge is assessing whether decentralized policy action is consistent with and can lead to total mitigation efforts that are effective, equitable, and efficient [6.1.2.1, 13.13]. 


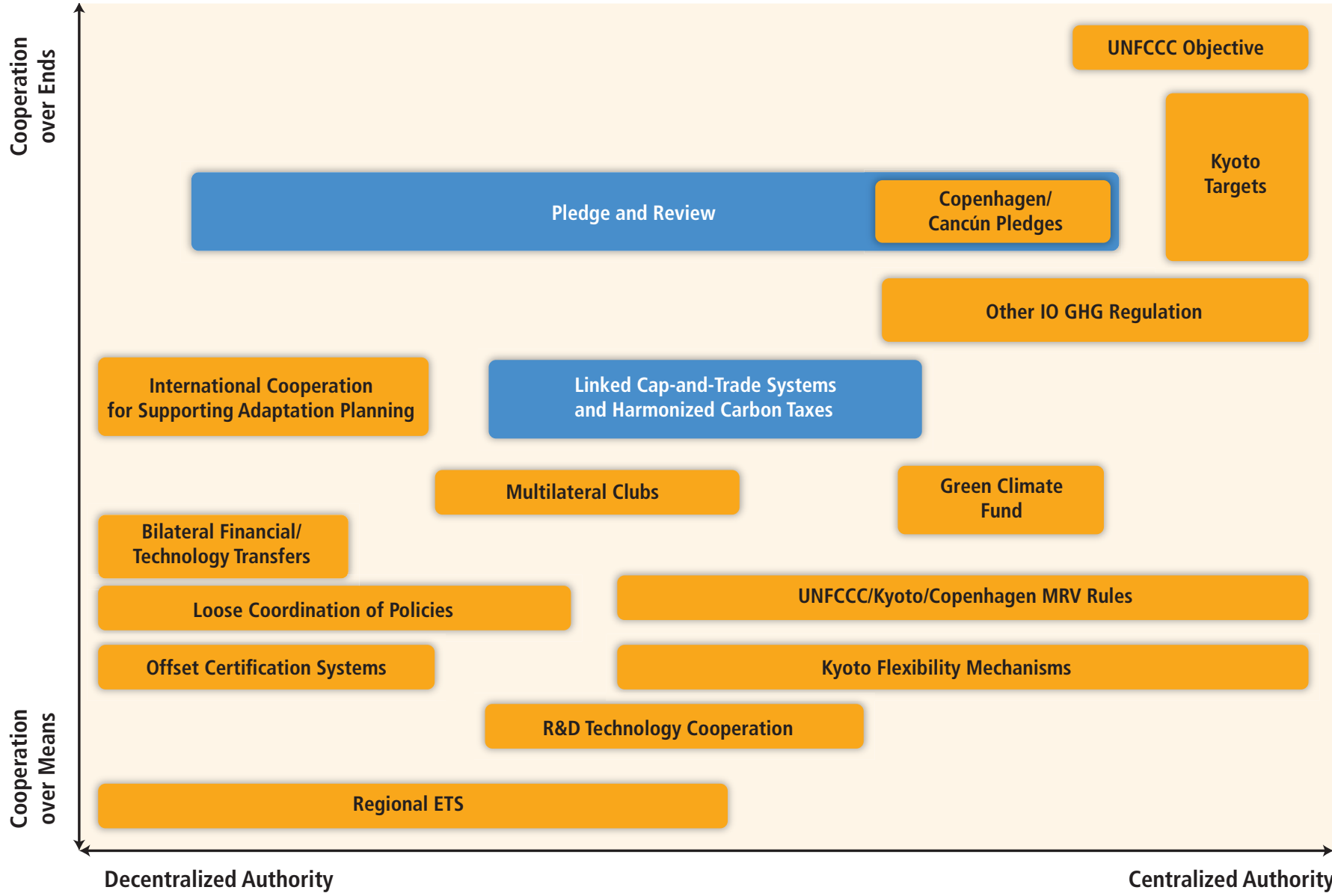

Loose coordination of policies: examples include transnational city networks and Nationally Appropriate Mitigation Actions (NAMAs); R\&D technology cooperation: examples include the Major Economies Forum on Energy and Climate (MEF), Global Methane Initiative (GMI), or Renewable Energy and Energy Efficiency Partnership (REEEP); Other international organization (IO) GHG regulation: examples include the Montreal Protocol, International Civil Aviation Organization (ICAO), International Maritime Organization (IMO).

Figure TS.38| Alternative forms of international cooperation. The figure represents a compilation of existing and possible forms of international cooperation, based upon a survey of published research, but is not intended to be exhaustive of existing or potential policy architectures, nor is it intended to be prescriptive. Examples in orange are existing agreements. Examples in blue are structures for agreements proposed in the literature. The width of individual boxes indicates the range of possible degrees of centralization for a particular agreement. The degree of centralization indicates the authority an agreement confers on an international institution, not the process of negotiating the agreement. [Figure 13.2]

International cooperation on climate change has become more institutionally diverse over the past decade (very high confidence). Perceptions of fairness can facilitate cooperation by increasing the legitimacy of an agreement [3.10, 13.2.2.4]. UNFCCC remains a primary international forum for climate negotiations, but other institutions have emerged at multiple scales, namely: global, regional, national, and local [13.3.1, 13.4.1.4, 13.5]. This institutional diversity arises in part from the growing inclusion of climate change issues in other policy arenas (e.g., sustainable development, international trade, and human rights). These and other linkages create opportunities, potential co-benefits, or harms that have not yet been thoroughly examined. Issue linkage also creates the possibility for countries to experiment with different forums of cooperation ('forum shopping'), which may increase negotiation costs and potentially distract from or dilute the performance of international cooperation toward climate goals. $[13.3,13.4,13.5]$ Finally, there has been an emergence of new transnational climate-related institutions not centred on sovereign states (e.g., public-private partnerships, private sector governance initiatives, transnational NGO programmes, and city level initiatives) [13.3.1, 13.12].

Existing and proposed international climate agreements vary in the degree to which their authority is centralized. As illustrated in Figure TS.38, the range of centralized formalization spans strong multilateral agreements (such as the Kyoto Protocol targets), harmonized national policies (such as the Copenhagen/Cancún pledges), and decentralized but coordinated national policies (such as planned linkages of national and sub-national emissions trading schemes) [13.4.1, 13.4.3]. Four other design elements of international agreements have particular relevance: legal bindingness, goals and targets, flexible mechanisms, and equitable methods for effort-shar- 
Table TS.10 | Summary of performance assessments of existing and proposed forms of cooperation. Forms of cooperation are evaluated along the four evaluation criteria described in Sections 3.7.1 and 13.2.2. [Table 13.3]

\begin{tabular}{|c|c|c|c|c|c|c|}
\hline & \multirow{2}{*}{\multicolumn{2}{|c|}{$\begin{array}{l}\text { Mode of International } \\
\text { Cooperation }\end{array}$}} & \multicolumn{4}{|c|}{ Assessment Criteria } \\
\hline & & & $\begin{array}{l}\text { Environmental } \\
\text { Effectiveness }\end{array}$ & $\begin{array}{l}\text { Aggregate Economic } \\
\text { Performance }\end{array}$ & Distributional Impacts & Institutional Feasibility \\
\hline \multirow[t]{7}{*}{$\begin{array}{l}\text { Existing } \\
\text { Cooperation } \\
{[13.13 .1]}\end{array}$} & \multicolumn{2}{|l|}{ UNFCCC } & $\begin{array}{l}\text { Aggregate GHG emis- } \\
\text { sions in Annex I countries } \\
\text { declined by } 6.0 \text { to } 9.2 \% \\
\text { below } 1990 \text { levels by } 2000 \text {, } \\
\text { a larger reduction than the } \\
\text { apparent 'aim' of returning } \\
\text { to } 1990 \text { levels by } 2000 \text {. }\end{array}$ & $\begin{array}{l}\text { Authorized joint fulfilment } \\
\text { of commitments, multi-gas } \\
\text { approach, sources and sinks, } \\
\text { and domestic policy choice. } \\
\text { Cost and benefit estimates } \\
\text { depend on baseline, discount } \\
\text { rate, participation, leak- } \\
\text { age, co-benefits, adverse } \\
\text { effects, and other factors. }\end{array}$ & $\begin{array}{l}\text { Commitments distinguish } \\
\text { between Annex I (indus- } \\
\text { trialized) and non-Annex I } \\
\text { countries. Principle of } \\
\text { 'common but differentiated } \\
\text { responsibility.' Commitment } \\
\text { to 'equitable and appropriate } \\
\text { contributions by each [party].' }\end{array}$ & $\begin{array}{l}\text { Ratified (or equivalent) by } 195 \\
\text { countries and regional organi- } \\
\text { zations. Compliance depends } \\
\text { on national communications. }\end{array}$ \\
\hline & \multicolumn{2}{|c|}{ The Kyoto Protocol (KP) } & $\begin{array}{l}\text { Aggregate emissions in Annex I } \\
\text { countries were reduced by } 8.5 \\
\text { to } 13.6 \% \text { below } 1990 \text { levels by } \\
2011 \text {, more than the first com- } \\
\text { mitment period (CP1) collective } \\
\text { reduction target of } 5.2 \% \text {. Reduc- } \\
\text { tions occurred mainly in ElTs; } \\
\text { emissions; increased in some } \\
\text { others. Incomplete participation } \\
\text { in CP1 (even lower in CP2). }\end{array}$ & $\begin{array}{l}\text { Cost-effectiveness improved } \\
\text { by flexible mechanisms (Joint } \\
\text { Implementation (JI), CDM, } \\
\text { International Emissions } \\
\text { Trading (IET)) and domestic } \\
\text { policy choice. Cost and benefit } \\
\text { estimates depend on baseline, } \\
\text { discount rate, participation, } \\
\text { leakage, co-benefits, adverse } \\
\text { effects, and other factors. }\end{array}$ & $\begin{array}{l}\text { Commitments distinguish } \\
\text { between developed and } \\
\text { developing countries, but } \\
\text { dichotomous distinction } \\
\text { correlates only partly (and } \\
\text { decreasingly) with historical } \\
\text { emissions trends and with } \\
\text { changing economic circum- } \\
\text { stances. Intertemporal equity } \\
\text { affected by short-term actions. }\end{array}$ & $\begin{array}{l}\text { Ratified (or equivalent) by } \\
192 \text { countries and regional } \\
\text { organizations, but took } 7 \text { years } \\
\text { to enter into force. Compli- } \\
\text { ance depends on national } \\
\text { communications, plus KP } \\
\text { compliance system. Later } \\
\text { added approaches to enhance } \\
\text { measurement, reporting, } \\
\text { and verification (MRV). }\end{array}$ \\
\hline & \multicolumn{2}{|c|}{ The Kyoto Mechanisms } & $\begin{array}{l}\text { About } 1.4 \text { billion } \mathrm{tCO}_{2} \mathrm{eq} \\
\text { credits under the } \mathrm{CDM} 0.8 \\
\text { billion under JI, and } 0.2 \text { bil- } \\
\text { lion under IET (through July } \\
\text { 2013). Additionality of CDM } \\
\text { projects remains an issue but } \\
\text { regulatory reform underway. }\end{array}$ & $\begin{array}{l}\text { CDM mobilized low cost } \\
\text { options, particularly indus- } \\
\text { trial gases, reducing costs. } \\
\text { Underperformance of some } \\
\text { project types. Some evidence } \\
\text { that technology is transferred } \\
\text { to non-Annex I countries. }\end{array}$ & $\begin{array}{l}\text { Limited direct investment from } \\
\text { Annex I countries. Domestic } \\
\text { investment dominates, leading } \\
\text { to concentration of CDM } \\
\text { projects in few countries. } \\
\text { Limited contributions to local } \\
\text { sustainable development. }\end{array}$ & $\begin{array}{l}\text { Helped enable political } \\
\text { feasibility of Kyoto Protocol. } \\
\text { Has multi-layered governance. } \\
\text { Largest carbon markets to date. } \\
\text { Has built institutional capacity } \\
\text { in developing countries. }\end{array}$ \\
\hline & \multicolumn{2}{|c|}{$\begin{array}{l}\text { Further Agreements } \\
\text { under the UNFCCC }\end{array}$} & $\begin{array}{l}\text { Pledges to limit emissions made } \\
\text { by all major emitters under } \\
\text { Cancun Agreements. Unlikely } \\
\text { sufficient to limit temperature } \\
\text { change to } 2^{\circ} \mathrm{C} \text {. Depends on } \\
\text { treatment of measures beyond } \\
\text { current pledges for mitigation } \\
\text { and finance. Durban Platform } \\
\text { calls for new agreement } \\
\text { by } 2015 \text {, to take effect in } \\
2020 \text {, engaging all parties. }\end{array}$ & $\begin{array}{l}\text { Efficiency not assessed. } \\
\text { Cost-effectiveness might be } \\
\text { improved by market-based } \\
\text { policy instruments, inclusion of } \\
\text { forestry sector, commitments } \\
\text { by more nations than Annex I } \\
\text { countries (as envisioned } \\
\text { in Durban Platform). }\end{array}$ & $\begin{array}{l}\text { Depends on sources of financ- } \\
\text { ing, particularly for actions } \\
\text { of developing countries. }\end{array}$ & $\begin{array}{l}\text { Cancún Conference of the } \\
\text { Parties (COP) decision; } 97 \\
\text { countries made pledges of } \\
\text { emission reduction targets } \\
\text { or actions for } 2020 \text {. }\end{array}$ \\
\hline & \multirow[t]{3}{*}{$\begin{array}{l}\text { Agreements } \\
\text { outside the } \\
\text { UNFCCC }\end{array}$} & $\begin{array}{l}\text { G8, G20, } \\
\text { Major } \\
\text { Economies } \\
\text { Forum on } \\
\text { Energy and } \\
\text { Climate (MEF) }\end{array}$ & $\begin{array}{l}\text { G8 and MEF have recom- } \\
\text { mended emission reduction by } \\
\text { all major emitters. G20 may } \\
\text { spur GHG reductions by phas- } \\
\text { ing out of fossil fuel subsidies. }\end{array}$ & $\begin{array}{l}\text { Action by all major emitters } \\
\text { may reduce leakage and } \\
\text { improve cost-effectiveness, if } \\
\text { implemented using flexible } \\
\text { mechanisms. Potential efficiency } \\
\text { gains through subsidy removal. } \\
\text { Too early to assess economic } \\
\text { performance empirically. }\end{array}$ & $\begin{array}{l}\text { Has not mobilized climate } \\
\text { finance. Removing fuel } \\
\text { subsidies would be progressive } \\
\text { but have negative effects } \\
\text { on oil-exporting countries } \\
\text { and on those with very low } \\
\text { incomes unless other help } \\
\text { for the poorest is provided. }\end{array}$ & $\begin{array}{l}\text { Lower participation of countries } \\
\text { than UNFCCC, yet covers } 70 \% \\
\text { of global emissions. Opens } \\
\text { possibility for forum-shopping, } \\
\text { based on issue preferences. }\end{array}$ \\
\hline & & $\begin{array}{l}\text { Montreal } \\
\text { Protocol on } \\
\text { Ozone- } \\
\text { Depleting } \\
\text { Substances } \\
\text { (ODS) }\end{array}$ & $\begin{array}{l}\text { Spurred emission reductions } \\
\text { through ODS phaseouts } \\
\text { approximately } 5 \text { times the } \\
\text { magnitude of Kyoto CP1 } \\
\text { targets. Contribution may } \\
\text { be negated by high-GWP } \\
\text { substitutes, though efforts to } \\
\text { phase out HFCs are growing. }\end{array}$ & $\begin{array}{l}\text { Cost-effectiveness supported } \\
\text { by multi-gas approach. Some } \\
\text { countries used market-based } \\
\text { mechanisms to imple- } \\
\text { ment domestically. }\end{array}$ & $\begin{array}{l}\text { Later compliance period for } \\
\text { phaseouts by developing } \\
\text { countries. Montreal Protocol } \\
\text { Fund provided finance to } \\
\text { developing countries. }\end{array}$ & $\begin{array}{l}\text { Universal participation. } \\
\text { but the timing of required } \\
\text { actions vary for developed } \\
\text { and developing countries }\end{array}$ \\
\hline & & $\begin{array}{l}\text { Voluntary } \\
\text { Carbon } \\
\text { Market }\end{array}$ & $\begin{array}{l}\text { Covers } 0.13 \text { billion } \mathrm{tCO}_{2} \text { eq, but } \\
\text { certification remains an issue }\end{array}$ & $\begin{array}{l}\text { Credit prices are het- } \\
\text { erogeneous, indicating } \\
\text { market inefficiencies }\end{array}$ & [No literature cited.] & $\begin{array}{l}\text { Fragmented and non- } \\
\text { transparent market. }\end{array}$ \\
\hline
\end{tabular}




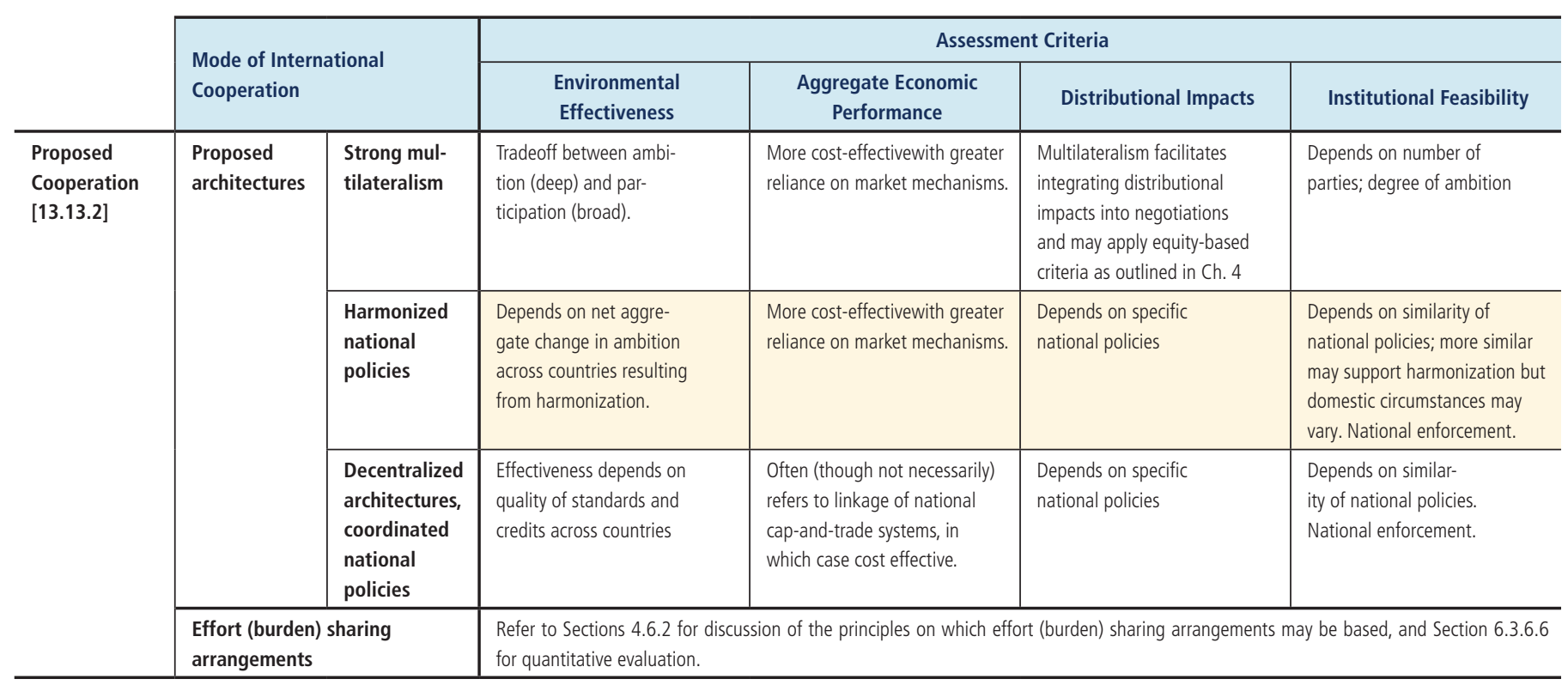

ing [13.4.2]. Existing and proposed modes of international cooperation are assessed in Table TS.10. [13.13]

The UNFCCC is currently the only international climate policy venue with broad legitimacy, due in part to its virtually universal membership (high confidence). The UNFCCC continues to evolve institutions and systems for governance of climate change. [13.2.2.4, $13.3 .1,13.4 .1 .4,13.5]$

Incentives for international cooperation can interact with other policies (medium confidence). Interactions between proposed and existing policies, which may be counterproductive, inconsequential, or beneficial, are difficult to predict, and have been understudied in the literature $[13.2,13.13,15.7 .4]$. The game-theoretic literature on climate change agreements finds that self-enforcing agreements engage and maintain participation and compliance. Self-enforcement can be derived from national benefits due to direct climate benefits, co-benefits of mitigation on other national objectives, technology transfer, and climate finance. [13.3.2]

Decreasing uncertainty concerning the costs and benefits of mitigation can reduce the willingness of states to make commitments in forums of international cooperation (medium confidence). In some cases, the reduction of uncertainty concerning the costs and benefits of mitigation can make international agreements less effective by creating a disincentive for states to participate [13.3.3, 2.6.4.1]. A second dimension of uncertainty, that concerning whether the policies states implement will in fact achieve desired outcomes, can lessen the willingness of states to agree to commitments regarding those outcomes [2.6.3].

International cooperation can stimulate public and private investment and the adoption of economic incentives and direct regulations that promote technological innovation (medium confidence). Technology policy can help lower mitigation costs, thereby increasing incentives for participation and compliance with international cooperative efforts, particularly in the long run. Equity issues can be affected by domestic intellectual property rights regimes, which can alter the rate of both technology transfer and the development of new technologies. $[13.3,13.9]$

In the absence of-or as a complement to-a binding, international agreement on climate change, policy linkages between and among existing and nascent international, regional, national, and sub-national climate policies offer potential climate change mitigation and adaptation benefits (medium confidence). Direct and indirect linkages between and among sub-national, national, and regional carbon markets are being pursued to improve market efficiency. Linkage between carbon markets can be stimulated by competition between and among public and private governance regimes, accountability measures, and the desire to learn from policy experiments. Yet integrating climate policies raises a number of concerns about the performance of a system of linked legal rules and economic activities. [13.3.1, 13.5.3, 13.13.2.3] Prominent examples of linkages are among national and regional climate initiatives (e.g., planned linkage between the EU ETS and the Australian Emission Trading Scheme, international offsets planned for recognition by a number of jurisdictions), and national and regional climate initiatives with the Kyoto Protocol (e.g., the EU ETS is linked to international carbon markets through the project-based Kyoto Mechanisms) [13.6, 13.7, Figure 13.4, 14.4.2].

International trade can promote or discourage international cooperation on climate change (high confidence). Developing constructive relationships between international trade and climate agreements involves considering how existing trade policies and rules 
can be modified to be more climate-friendly; whether border adjustment measures or other trade measures can be effective in meeting the goals of international climate policy, including participation in and compliance with climate agreements; or whether the UNFCCC, World Trade Organization (WTO), a hybrid of the two, or a new institution is the best forum for a trade-and-climate architecture. [13.8]

The Montreal Protocol, aimed at protecting the stratospheric ozone layer, achieved reductions in global GHG emissions (very high confidence). The Montreal Protocol set limits on emissions of ozone-depleting gases that are also potent GHGs, such as chlorofluorocarbons (CFCs) and hydrochlorofluorocarbons (HCFCs). Substitutes for those ozone-depleting gases (such as hydrofluorocarbons (HFCs), which are not ozone-depleting) may also be potent GHGs. Lessons learned from the Montreal Protocol, for example about the effect of financial and technological transfers on broadening participation in an international environmental agreement, could be of value to the design of future international climate change agreements (see Table TS.10). [13.3.3, 13.3.4, 13.13.1.4]

The Kyoto Protocol was the first binding step toward implementing the principles and goals provided by the UNFCCC, but it has had limited effects on global GHG emissions because some countries did not ratify the Protocol, some Parties did not meet their commitments, and its commitments applied to only a portion of the global economy (medium evidence, low agreement). The Parties collectively surpassed their collective emission reduction target in the first commitment period, but the Protocol credited emissions reductions that would have occurred even in its absence. The Kyoto Protocol does not directly influence the emissions of non-Annex I countries, which have grown rapidly over the past decade. [5.2, 13.13.1.1]

The flexible mechanisms under the Protocol have cost-saving potential, but their environmental effectiveness is less clear (medium confidence). The CDM, one of the Protocol's flexible mechanisms, created a market for GHG emissions offsets from developing countries, generating credits equivalent to nearly $1.4 \mathrm{GtCO}_{2} \mathrm{eq}$ as of October 2013. The CDM's environmental effectiveness has been mixed due to concerns about the limited additionality of projects, the validity of baselines, the possibility of emissions leakage, and recent credit price decreases. Its distributional impact has been unequal due to the concentration of projects in a limited number of countries. The Protocol's other flexible mechanisms, Joint Implementation (JI) and International Emissions Trading (IET), have been undertaken both by governments and private market participants, but have raised concerns related to government sales of emission units. (Table TS.10) [13.7.2, 13.13.1.2, 14.3.7.1]

Recent UNFCCC negotiations have sought to include more ambitious contributions from the countries with commitments under the Kyoto Protocol, mitigation contributions from a broader set of countries, and new finance and technology mechanisms.
Under the 2010 Cancún Agreement, developed countries formalized voluntary pledges of quantified, economy-wide GHG emission reduction targets and some developing countries formalized voluntary pledges to mitigation actions. The distributional impact of the agreement will depend in part on the magnitude and sources of financing, although the scientific literature on this point is limited, because financing mechanisms are evolving more rapidly than respective scientific assessments (limited evidence, low agreement). Under the 2011 Durban Platform for Enhanced Action, delegates agreed to craft a future legal regime that would be 'applicable to all Parties [...] under the Convention' and would include substantial new financial support and technology arrangements to benefit developing countries, but the delegates did not specify means for achieving those ends. [13.5.1.1, 13.13.1.3, 16.2.1]

\section{TS.4.5 Investment and finance}

A transformation to a low-carbon economy implies new patterns of investment. A limited number of studies have examined the investment needs for different mitigation scenarios. Information is largely limited to energy use with global total annual investment in the energy sector at about 1200 billion USD. Mitigation scenarios that reach atmospheric $\mathrm{CO}_{2}$ eq concentrations in the range from 430 to 530 ppm $\mathrm{CO}_{2}$ eq by 2100 (without overshoot) show substantial shifts in annual investment flows during the period 2010-2029 if compared to baseline scenarios (Figure TS.39): annual investment in the existing technologies associated with the energy supply sector (e.g., conventional fossil fuelled power plants and fossil fuel extraction) would decline by 30 (2 to 166) billion USD per year (median:-20\% compared to 2010) (limited evidence, medium agreement). Investment in lowemissions generation technologies (renewables, nuclear, and power plants with CCS) would increase by 147 (31 to 360) billion USD per year (median: $+100 \%$ compared to 2010 ) during the same period (limited evidence, medium agreement) in combination with an increase by 336 (1 to 641) billion USD in energy efficiency investments in the building, transport and industry sectors (limited evidence, medium agreement). Higher energy efficiency and the shift to low-emission generation technologies contribute to a reduction in the demand for fossil fuels, thus causing a decline in investment in fossil fuel extraction, transformation and transportation. Scenarios suggest that average annual reduction of investment in fossil fuel extraction in 2010-2029 would be 116 (-8 to 369) billion USD (limited evidence, medium agreement). Such spillover effects could yield adverse effects on the revenues of countries that export fossil fuels. Mitigation scenarios also reduce deforestation against current deforestation trends by $50 \%$ reduction with an investment of 21 to 35 billion USD per year (low confidence). [16.2.2]

Estimates of total climate finance range from 343 to 385 billion USD per year between 2010 and 2012 (medium confidence). The range is based on 2010, 2011, and 2012 data. Climate finance was almost evenly invested in developed and developing countries. Around $95 \%$ of the total was invested in mitigation (medium confidence). The 
figures reflect the total financial flow for the underlying investments, not the incremental investment, i.e., the portion attributed to the mitigation/adaptation cost increment (see Box TS.14). In general, quantitative data on climate finance are limited, relate to different concepts, and are incomplete. [16.2.1.1]

Depending on definitions and approaches, climate finance flows to developing countries are estimated to range from 39 to 120 billion USD per year during the period 2009 to 2012 (medium confidence). The range covers public and private flows for mitigation and adaptation. Public climate finance was 35 to 49 billion USD (2011/2012 USD) (medium confidence). Most public climate finance provided to developing countries flows through bilateral and multilateral institutions usually as concessional loans and grants. Under the UNFCCC, climate finance is funding provided to developing countries by Annex II Parties and averaged nearly 10 billion USD per year from
2005 to 2010 (medium confidence). Between 2010 and 2012, the 'fast start finance' provided by some developed countries amounted to over 10 billion USD per year (medium confidence). Estimates of international private climate finance flowing to developing countries range from 10 to 72 billion USD (2009/2010 USD) per year, including foreign direct investment as equity and loans in the range of 10 to 37 billion USD (2010 USD and 2008 USD) per year over the period of 2008-2011 (medium confidence). Figure TS.40 provides an overview of climate finance, outlining sources and managers of capital, financial instruments, project owners, and projects. [16.2.1.1]

Within appropriate enabling environments, the private sector, along with the public sector, can play an important role in financing mitigation. The private sector contribution to total climate finance is estimated at an average of 267 billion USD (74\%) per year in the period 2010 to 2011 and at 224 billion USD (62\%) per year in the

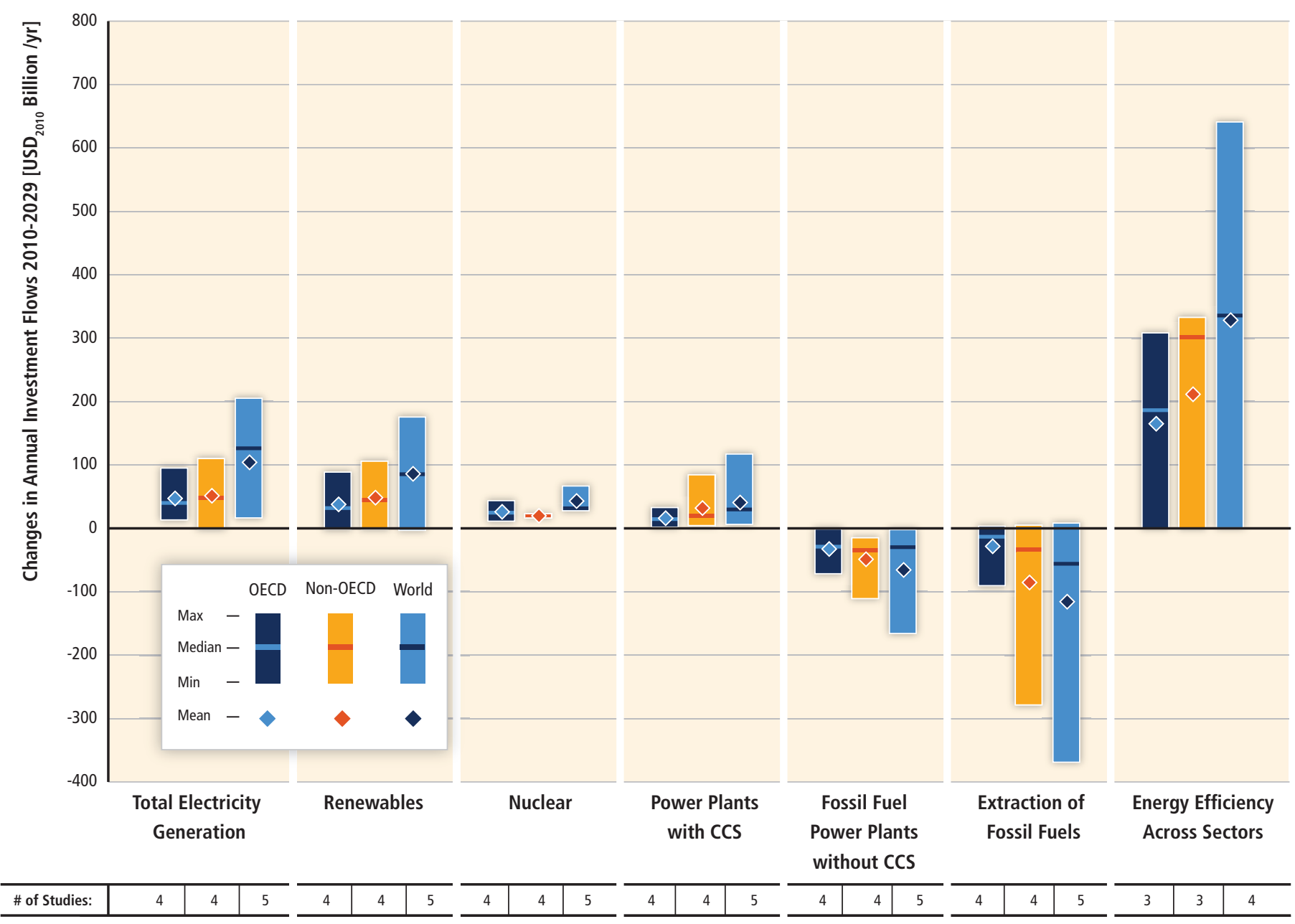

Figure TS.39| Change of average annual investment flows in mitigation scenarios (2010-2029). Investment changes are calculated by a limited number of model studies and model comparisons for mitigation scenarios that reach concentrations within the range of $430-530$ ppm $\mathrm{CO}_{2}$ eq by 2100 compared to respective average baseline investments. The vertical bars indicate the range between minimum and maximum estimate of investment changes; the horizontal bar indicates the median of model results. Proximity to this median value does not imply higher likelihood because of the different degree of aggregation of model results, low number of studies available and different assumptions in the different studies considered. The numbers in the bottom row show the total number of studies assessed. [Figure 16.3] 


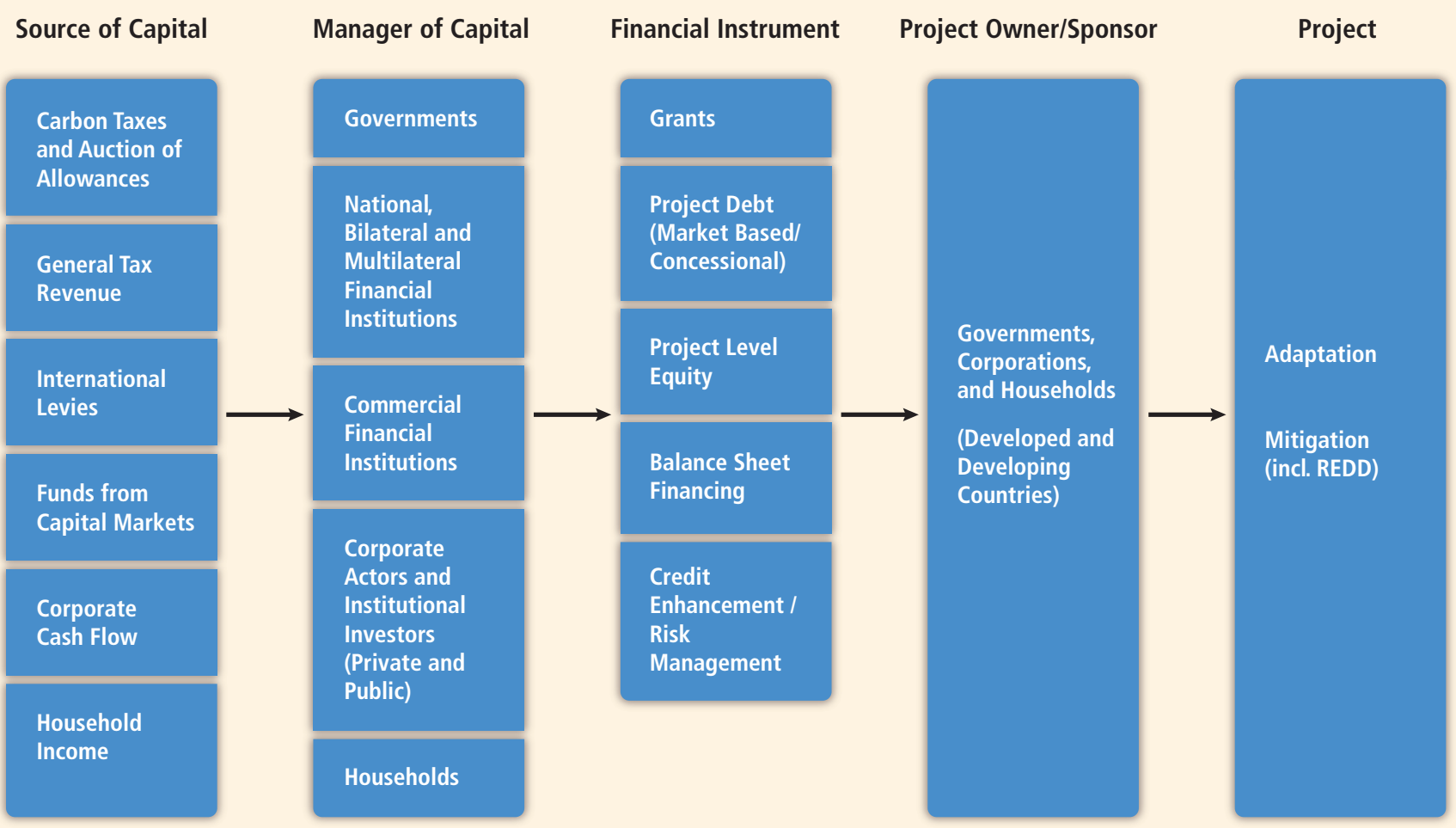

Figure TS.40| Types of climate finance flows. 'Capital' includes all relevant financial flows. The size of the boxes is not related to the magnitude of the financial flow. [Figure 16.1]

\section{Box TS.14 | There are no agreed definitions of 'climate investment' and 'climate finance'}

'Total climate finance' includes all financial flows whose expected effect is to reduce net GHG emissions and/or to enhance resilience to the impacts of climate variability and the projected climate change. This covers private and public funds, domestic and international flows, expenditures for mitigation and adaptation, and adaptation to current climate variability as well as future climate change. It covers the full value of the financial flow rather than the share associated with the climate change benefit. The share associated with the climate change benefit is the incremental cost. The 'total climate finance flowing to developing countries' is the amount of the total climate finance invested in developing countries that comes from developed countries. This covers private and public funds for mitigation and adaptation. 'Public climate finance provided to developing countries' is the finance provided by developed countries' governments and bilateral institutions as well as multilateral institutions for mitigation and adaptation activities in developing countries. 'Private climate finance flowing to developing countries' is finance and investment by private actors in/from developed countries for mitigation and adaptation activities in developing countries. Under the UNFCCC, climate finance is not well-defined. Annex II Parties provide and mobilize funding for climate-related activities in developing countries.
The 'incremental investment' is the extra capital required for the initial investment for a mitigation or adaptation project in comparison to a reference project. Incremental investment for mitigation and adaptation projects is not regularly estimated and reported, but estimates are available from models. The 'incremental cost' reflects the cost of capital of the incremental investment and the change of operating and maintenance costs for a mitigation or adaptation project in comparison to a reference project. It can be calculated as the difference of the net present values of the two projects. Many mitigation measures have higher investment costs and lower operating and maintenance costs than the measures displaced so incremental cost tends to be lower than the incremental investment. Values depend on the incremental investment as well as projected operating costs, including fossil fuel prices, and the discount rate. The 'macroeconomic cost of mitigation policy' is the reduction of aggregate consumption or GDP induced by the reallocation of investments and expenditures induced by climate policy (see Box TS.9). These costs do not account for the benefit of reducing anthropogenic climate change and should thus be assessed against the economic benefit of avoided climate change impacts. [16.1] 
period 2011 to 2012 (limited evidence, medium agreement) [16.2.1]. In a range of countries, a large share of private sector climate investment relies on low-interest and long-term loans as well as risk guarantees provided by public sector institutions to cover the incremental costs and risks of many mitigation investments. The quality of a country's enabling environment-including the effectiveness of its institutions, regulations and guidelines regarding the private sector, security of property rights, credibility of policies, and other factors-has a substantial impact on whether private firms invest in new technologies and infrastructure [16.3]. By the end of 2012, the 20 largest emitting developed and developing countries with lower risk country grades for private sector investments produced $70 \%$ of global energy related $\mathrm{CO}_{2}$ emissions (low confidence). This makes them attractive for international private sector investment in low-carbon technologies. In many other countries, including most least-developed countries, low-carbon investment will often have to rely mainly on domestic sources or international public finance. [16.4.2]
A main barrier to the deployment of low-carbon technologies is a low risk-adjusted rate of return on investment vis-à-vis high-carbon alternatives (high confidence). Public policies and support instruments can address this either by altering the average rates of return for different investment options, or by creating mechanisms to lessen the risks that private investors face [15.12, 16.3]. Carbon pricing mechanisms (carbon taxes, cap-and-trade systems), as well as renewable energy premiums, FITs, RPSs, investment grants, soft loans and credit insurance can move risk-return profiles into the required direction [16.4]. For some instruments, the presence of substantial uncertainty about their future levels (e.g., the future size of a carbon tax relative to differences in investment and operating costs) can lead to a lessening of the effectiveness and/or efficiency of the instrument. Instruments that create a fixed or immediate incentive to invest in low-emission technologies, such as investment grants, soft loans, or FITs, do not appear to suffer from this problem. [2.6.5] 



\section{Annex}





\section{Glossary, Acronyms and Chemical Symbols}

\section{Glossary Editors:}

Julian M. Allwood (UK), Valentina Bosetti (Italy), Navroz K. Dubash (India), Luis Gómez-Echeverri (Austria/Colombia), Christoph von Stechow (Germany)

\section{Glossary Contributors:}

Marcio D’Agosto (Brazil), Giovanno Baiocchi (UK/Italy), John Barrett (UK), John Broome (UK), Steffen Brunner (Germany), Micheline Cariño Olvera (Mexico), Harry Clark (New Zealand), Leon Clarke (USA), Heleen C. de Coninck (Netherlands), Esteve Corbera (Spain), Felix Creutzig (Germany), Gian Carlo Delgado (Mexico), Manfred Fischedick (Germany), Marc Fleurbaey (France/USA), Don Fullerton (USA), Richard Harper (Australia), Edgar Hertwich (Austria/Norway), Damon Honnery (Australia), Michael Jakob (Germany), Charles Kolstad (USA), Elmar Kriegler (Germany), Howard Kunreuther (USA), Andreas Löschel (Germany), Oswaldo Lucon (Brazil), Axel Michaelowa (Germany/Switzerland), Jan C. Minx (Germany), Luis Mundaca (Chile/Sweden), Jin Murakami (Japan/China), Jos G.J. Olivier (Netherlands), Michael Rauscher (Germany), Keywan Riahi (Austria), H.-Holger Rogner (Germany), Steffen Schlömer (Germany), Ralph Sims (New Zealand), Pete Smith (UK), David I. Stern (Australia), Neil Strachan (UK), Kevin Urama (Nigeria/UK/Kenya), Diana Ürge-Vorsatz (Hungary), David G. Victor (USA), Elke Weber (USA), Jonathan Wiener (USA), Mitsutsune Yamaguchi (Japan), Azni Zain Ahmed (Malaysia)

This annex should be cited as:

Allwood J. M., V. Bosetti, N. K. Dubash, L. Gómez-Echeverri, and C. von Stechow, 2014: Glossary. In: Climate Change 2014: Mitigation of Climate Change. Contribution of Working Group III to the Fifth Assessment Report of the Intergovernmental Panel on Climate Change [Edenhofer, O., R. Pichs-Madruga, Y. Sokona, E. Farahani, S. Kadner, K. Seyboth, A. Adler, I. Baum, S. Brunner, P. Eickemeier, B. Kriemann, J. Savolainen, S. Schlömer, C. von Stechow, T. Zwickel and J.C. Minx (eds.)]. Cambridge University Press, Cambridge, United Kingdom and New York, NY, USA. 


\section{Contents}

Glossary.

Acronyms and chemical symbols

References 


\section{Glossary}

This glossary defines some specific terms as the Lead Authors intend them to be interpreted in the context of this report. Glossary entries (highlighted in bold) are by preference subjects; a main entry can contain subentries, in bold and italic, for example, Primary Energy is defined under the entry Energy. Blue, italicized words indicate that the term is defined in the Glossary. The glossary is followed by a list of acronyms and chemical symbols. Please refer to Annex II for standard units, prefixes, and unit conversion (Section A.II.1) and for regions and country groupings (Section A.II.2).

Abrupt climate change: A large-scale change in the climate system that takes place over a few decades or less, persists (or is anticipated to persist) for at least a few decades, and causes substantial disruptions in human and natural systems. See also Climate threshold.

Adaptability: See Adaptive capacity.

Adaptation: The process of adjustment to actual or expected climate and its effects. In human systems, adaptation seeks to moderate or avoid harm or exploit beneficial opportunities. In some natural systems, human intervention may facilitate adjustment to expected climate and its effects. ${ }^{1}$

Adaptation Fund: A Fund established under the Kyoto Protocol in 2001 and officially launched in 2007. The Fund finances adaptation projects and programmes in developing countries that are Parties to the Kyoto Protocol. Financing comes mainly from sales of Certified Emissions Reductions (CERs) and a share of proceeds amounting to $2 \%$ of the value of CERs issued each year for Clean Development Mechanism (CDM) projects. The Adaptation Fund can also receive funds from government, private sector, and individuals.

Adaptive capacity: The ability of systems, institutions, humans, and other organisms to adjust to potential damage, to take advantage of opportunities, or to respond to consequences. ${ }^{2}$

Additionality: Mitigation projects (e.g., under the Kyoto Mechanisms), mitigation policies, or climate finance are additional if they go beyond a business-as-usual level, or baseline. Additionality is required to guarantee the environmental integrity of project-based offset mechanisms, but difficult to establish in practice due to the counterfactual nature of the baseline.

Reflecting progress in science, this glossary entry differs in breadth and focus from the entry used in the Fourth Assessment Report and other IPCC reports.

This glossary entry builds from definitions used in previous IPCC reports and the Millennium Ecosystem Assessment (MEA, 2005).
Adverse side-effects: The negative effects that a policy or measure aimed at one objective might have on other objectives, without yet evaluating the net effect on overall social welfare. Adverse side-effects are often subject to uncertainty and depend on, among others, local circumstances and implementation practices. See also Co-benefits, Risk, and Risk tradeoff.

Aerosol: A suspension of airborne solid or liquid particles, with a typical size between a few nanometres and $10 \mu \mathrm{m}$ that reside in the atmosphere for at least several hours. For convenience the term aerosol, which includes both the particles and the suspending gas, is often used in this report in its plural form to mean aerosol particles. Aerosols may be of either natural or anthropogenic origin. Aerosols may influence climate in several ways: directly through scattering and absorbing radiation, and indirectly by acting as cloud condensation nuclei or ice nuclei, modifying the optical properties and lifetime of clouds. Atmospheric aerosols, whether natural or anthropogenic, originate from two different pathways: emissions of primary particulate matter (PM), and formation of secondary PM from gaseous precursors. The bulk of aerosols are of natural origin. Some scientists use group labels that refer to the chemical composition, namely: sea salt, organic carbon, black carbon ( $B C)$, mineral species (mainly desert dust), sulphate, nitrate, and ammonium. These labels are, however, imperfect as aerosols combine particles to create complex mixtures. See also Short-lived climate po/lutants (SLCPS).

Afforestation: Planting of new forests on lands that historically have not contained forests. Afforestation projects are eligible under a number of schemes including, among others, Joint Implementation (JI) and the Clean Development Mechanism (CDM) under the Kyoto Protocol for which particular criteria apply (e.g., proof must be given that the land was not forested for at least 50 years or converted to alternative uses before 31 December 1989).

For a discussion of the term forest and related terms such as afforestation, reforestation and deforestation, see the IPCC Special Report on Land Use, Land-Use Change and Forestry (IPCC, 2000). See also the report on Definitions and Methodological Options to Inventory Emissions from Direct Human-induced Degradation of Forests and Devegetation of Other Vegetation Types (IPCC, 2003).

Agreement: In this report, the degree of agreement is the level of concurrence in the literature on a particular finding as assessed by the authors. See also Evidence, Confidence, Likelihood, and Uncertainty.

Agricultural emissions: See Emissions.

Agriculture, Forestry and Other Land Use (AFOLU): Agriculture, Forestry and Other Land Use plays a central role for food security and sustainable development (SD). The main mitigation options within AFOLU involve one or more of three strategies: prevention of emissions to the atmosphere by conserving existing carbon pools in soils or vegetation or by reducing emissions of methane $\left(\mathrm{CH}_{4}\right)$ and nitrous 
oxide $\left(\mathrm{N}_{2} \mathrm{O}\right)$; sequestration - increasing the size of existing carbon pools, and thereby extracting carbon dioxide $\left(\mathrm{CO}_{2}\right)$ from the atmosphere; and substitution - substituting biological products for fossil fuels or energy-intensive products, thereby reducing $\mathrm{CO}_{2}$ emissions. Demand-side measures (e.g., by reducing losses and wastes of food, changes in human diet, or changes in wood consumption) may also play a role. FOLU (Forestry and Other Land Use) - also referred to as LULUCF (Land use, land-use change, and forestry) - is the subset of AFOLU emissions and removals of greenhouse gases (GHGs) resulting from direct human-induced land use, land-use change and forestry activities excluding agricultural emissions.

Albedo: The fraction of solar radiation reflected by a surface or object, often expressed as a percentage. Snow-covered surfaces have a high albedo, the albedo of soils ranges from high to low, and vegetationcovered surfaces and oceans have a low albedo. The earth's planetary albedo varies mainly through varying cloudiness, snow, ice, leaf area and land cover changes.

Alliance of Small Island States (AOSIS): The Alliance of Small Island States (AOSIS) is a coalition of small islands and low-lying coastal countries with a membership of 44 states and observers that share and are active in global debates and negotiations on the environment, especially those related to their vulnerability to the adverse effects of climate change. Established in 1990, AOSIS acts as an ad-hoc lobby and negotiating voice for small island development states (SIDS) within the United Nations including the United Nations Framework Convention on Climate Change (UNFCCC) climate change negotiations.

Ancillary benefits: See Co-benefits.

Annex I Parties/countries: The group of countries listed in Annex I to the United Nations Framework Convention on Climate Change (UNFCCC). Under Articles 4.2 (a) and 4.2 (b) of the UNFCCC, Annex I Parties were committed to adopting national policies and measures with the non-legally binding aim to return their greenhouse gas (GHG) emissions to 1990 levels by 2000 . The group is largely similar to the Annex B Parties to the Kyoto Protocol that also adopted emissions reduction targets for 2008-2012. By default, the other countries are referred to as Non-Annex I Parties.

Annex II Parties/countries: The group of countries listed in Annex II to the United Nations Framework Convention on Climate Change (UNFCCC). Under Article 4 of the UNFCCC, these countries have a special obligation to provide financial resources to meet the agreed full incremental costs of implementing measures mentioned under Article 12, paragraph 1 . They are also obliged to provide financial resources, including for the transfer of technology, to meet the agreed incremental costs of implementing measures covered by Article 12, paragraph 1 and agreed between developing country Parties and international entities referred to in Article 11 of the UNFCCC. This group of countries shall also assist countries that are particularly vulnerable to the adverse effects of climate change.
Annex B Parties/countries: The subset of Annex / Parties that have accepted greenhouse gas (GHG) emission reduction targets for the period 2008-2012 under Article 3 of the Kyoto Protocol. By default, the other countries are referred to as Non-Annex I Parties.

\section{Anthropogenic emissions: See Emissions.}

Assigned Amount (AA): Under the Kyoto Protocol, the AA is the quantity of greenhouse gas (GHG) emissions that an Annex B country has agreed to as its cap on its emissions in the first five-year commitment period (2008-2012). The AA is the country's total GHG emissions in 1990 multiplied by five (for the five-year commitment period) and by the percentage it agreed to as listed in Annex B of the Kyoto Protocol (e.g., $92 \%$ for the EU). See also Assigned Amount Unit (AAU).

Assigned Amount Unit (AAU): An AAU equals 1 tonne (metric ton) of $\mathrm{CO}_{2}$-equivalent emissions calculated using the Global Warming Potential (GWP). See also Assigned Amount (AA).

Atmosphere: The gaseous envelope surrounding the earth, divided into five layers - the troposphere which contains half of the earth's atmosphere, the stratosphere, the mesosphere, the thermosphere, and the exosphere, which is the outer limit of the atmosphere. The dry atmosphere consists almost entirely of nitrogen (78.1\% volume mixing ratio) and oxygen ( $20.9 \%$ volume mixing ratio), together with a number of trace gases, such as argon $(0.93 \%$ volume mixing ratio), helium and radiatively active greenhouse gases (GHGs) such as carbon dioxide $\left(\mathrm{CO}_{2}\right)$ (0.035\% volume mixing ratio) and ozone $\left(\mathrm{O}_{3}\right)$. In addition, the atmosphere contains the GHG water vapour $\left(\mathrm{H}_{2} \mathrm{O}\right)$, whose amounts are highly variable but typically around $1 \%$ volume mixing ratio. The atmosphere also contains clouds and aerosols.

Backstop technology: Models estimating mitigation often use an arbitrary carbon-free technology (often for power generation) that might become available in the future in unlimited supply over the horizon of the model. This allows modellers to explore the consequences and importance of a generic solution technology without becoming enmeshed in picking the actual technology. This 'backstop' technology might be a nuclear technology, fossil technology with Carbon Dioxide Capture and Storage (CCS), solar energy, or something as yet unimagined. The backstop technology is typically assumed either not to currently exist, or to exist only at higher costs relative to conventional alternatives.

Banking (of Assigned Amount Units): Any transfer of Assigned Amount Units (AAUs) from an existing period into a future commitment period. According to the Kyoto Protocol [Article 3 (13)], Parties included in Annex I to the United Nations Framework Convention on Climate Change (UNFCCC) may save excess AAUs from the first commitment period for compliance with their respective cap in subsequent commitment periods (post-2012). 
Baseline/reference: The state against which change is measured. In the context of transformation pathways, the term 'baseline scenarios' refers to scenarios that are based on the assumption that no mitigation policies or measures will be implemented beyond those that are already in force and/or are legislated or planned to be adopted. Baseline scenarios are not intended to be predictions of the future, but rather counterfactual constructions that can serve to highlight the level of emissions that would occur without further policy effort. Typically, baseline scenarios are then compared to mitigation scenarios that are constructed to meet different goals for greenhouse gas $(G H G)$ emissions, atmospheric concentrations, or temperature change. The term 'baseline scenario' is used interchangeably with 'reference scenario' and 'no policy scenario'. In much of the literature the term is also synonymous with the term 'business-as-usual (BAU) scenario,' although the term 'BAU' has fallen out of favour because the idea of 'business-as-usual' in century-long socioeconomic projections is hard to fathom. See also Climate scenario, Emission scenario, Representative concentration pathways (RCPs), Shared socio-economic pathways, Socio-economic scenarios, SRES scenarios, and Stabilization.

Behaviour: In this report, behaviour refers to human decisions and actions (and the perceptions and judgments on which they are based) that directly or indirectly influence mitigation or the effects of potential climate change impacts (adaptation). Human decisions and actions are relevant at different levels, from international, national, and subnational actors, to NGO, tribe, or firm-level decision makers, to communities, households, and individual citizens and consumers. See also Behavioural change and Drivers of behaviour.

Behavioural change: In this report, behavioural change refers to alteration of human decisions and actions in ways that mitigate climate change and/or reduce negative consequences of climate change impacts. See also Drivers of behaviour.

Biochar: Biomass stabilization can be an alternative or enhancement to bioenergy in a land-based mitigation strategy. Heating biomass with exclusion of air produces a stable carbon-rich co-product (char). When added to soil a system, char creates a system that has greater abatement potential than typical bioenergy. The relative benefit of biochar systems is increased if changes in crop yield and soil emissions of methane $\left(\mathrm{CH}_{4}\right)$ and nitrous oxide $\left(\mathrm{N}_{2} \mathrm{O}\right)$ are taken into account.

Biochemical oxygen demand (BOD): The amount of dissolved oxygen consumed by micro-organisms (bacteria) in the bio-chemical oxidation of organic and inorganic matter in wastewater. See also Chemical oxygen demand (COD).

Biodiversity: The variability among living organisms from terrestrial, marine, and other ecosystems. Biodiversity includes variability at the genetic, species, and ecosystem levels. ${ }^{3}$

This glossary entry builds from definitions used in the Global Biodiversity Assessment (Heywood, 1995) and the Millennium Ecosystem Assessment (MEA, 2005).
Bioenergy: Energy derived from any form of biomass such as recently living organisms or their metabolic by-products.

Bioenergy and Carbon Dioxide Capture and Storage (BECCS): The application of Carbon Dioxide Capture and Storage (CCS) technology to bioenergy conversion processes. Depending on the total lifecycle emissions, including total marginal consequential effects (from indirect land use change (iLUC) and other processes), BECCS has the potential for net carbon dioxide $\left(\mathrm{CO}_{2}\right)$ removal from the atmosphere. See also Sequestration.

Bioethanol: Ethanol produced from biomass (e.g., sugar cane or corn). See also Biofuel.

Biofuel: A fuel, generally in liquid form, produced from organic matter or combustible oils produced by living or recently living plants. Examples of biofuel include alcohol (bioethanol), black liquor from the paper-manufacturing process, and soybean oil.

First-generation manufactured biofuel: First-generation manufactured biofuel is derived from grains, oilseeds, animal fats, and waste vegetable oils with mature conversion technologies.

Second-generation biofuel: Second-generation biofuel uses non-traditional biochemical and thermochemical conversion processes and feedstock mostly derived from the lignocellulosic fractions of, for example, agricultural and forestry residues, municipal solid waste, etc.

Third-generation biofuel: Third-generation biofuel would be derived from feedstocks such as algae and energy crops by advanced processes still under development.

These second- and third-generation biofuels produced through new processes are also referred to as next-generation or advanced biofuels, or advanced biofuel technologies.

Biomass: The total mass of living organisms in a given area or volume; dead plant material can be included as dead biomass. In the context of this report, biomass includes products, by-products, and waste of biological origin (plants or animal matter), excluding material embedded in geological formations and transformed to fossil fuels or peat.

Traditional biomass: Traditional biomass refers to the biomass-fuelwood, charcoal, agricultural residues, and animal dung - used with the so-called traditional technologies such as open fires for cooking, rustic kilns and ovens for small industries. Widely used in developing countries, where about 2.6 billion people cook with open wood fires, and hundreds of thousands smallindustries. The use of these rustic technologies leads to high pollution levels and, in specific circumstances, to forest degradation and deforestation. There are many successful initiatives around the world to make traditional biomass burned more efficiently 
and cleanly using efficient cookstoves and kilns. This last use of traditional biomass is sustainable and provides large health and economic benefits to local populations in developing countries, particularly in rural and peri-urban areas.

Modern biomass: All biomass used in high efficiency conversion systems.

Biomass burning: Biomass burning is the burning of living and dead vegetation.

Biosphere (terrestrial and marine): The part of the earth system comprising all ecosystems and living organisms, in the atmosphere, on land (terrestrial biosphere) or in the oceans (marine biosphere), including derived dead organic matter, such as litter, soil organic matter and oceanic detritus.

Black carbon (BC): Operationally defined aerosol species based on measurement of light absorption and chemical reactivity and/or thermal stability. It is sometimes referred to as soot. BC is mostly formed by the incomplete combustion of fossil fuels, biofuels, and biomass but it also occurs naturally. It stays in the atmosphere only for days or weeks. It is the most strongly light-absorbing component of particulate matter (PM) and has a warming effect by absorbing heat into the atmosphere and reducing the albedo when deposited on ice or snow.

Burden sharing (also referred to as Effort sharing): In the context of mitigation, burden sharing refers to sharing the effort of reducing the sources or enhancing the sinks of greenhouse gases (GHGs) from historical or projected levels, usually allocated by some criteria, as well as sharing the cost burden across countries.

\section{Business-as-usual (BAU): See Baseline/reference.}

Cancún Agreements: A set of decisions adopted at the 16th Session of the Conference of the Parties (COP) to the United Nations Framework Convention on Climate Change (UNFCCC), including the following, among others: the newly established Green Climate Fund (GCF), a newly established technology mechanism, a process for advancing discussions on adaptation, a formal process for reporting mitigation commitments, a goal of limiting global mean surface temperature increase to $2{ }^{\circ} \mathrm{C}$, and an agreement on MRV—Measuring, Reporting and Verifying for those countries that receive international support for their mitigation efforts.

Cancún Pledges: During 2010, many countries submitted their existing plans for controlling greenhouse gas (GHG) emissions to the Climate Change Secretariat and these proposals have now been formally acknowledged under the United Nations Framework Convention on Climate Change (UNFCCC). Developed countries presented their plans in the shape of economy-wide targets to reduce emissions, mainly up to 2020, while developing countries proposed ways to limit their growth of emissions in the shape of plans of action.
Cap, on emissions: Mandated restraint as an upper limit on emissions within a given period. For example, the Kyoto Protocol mandates emissions caps in a scheduled timeframe on the anthropogenic greenhouse gas (GHG) emissions released by Annex B countries.

Carbon budget: The area under a greenhouse gas (GHG) emissions trajectory that satisfies assumptions about limits on cumulative emissions estimated to avoid a certain level of global mean surface temperature rise. Carbon budgets may be defined at the global level, national, or sub-national levels.

\section{Carbon credit: See Emission allowance.}

Carbon cycle: The term used to describe the flow of carbon (in various forms, e. g., as carbon dioxide) through the atmosphere, ocean, terrestrial and marine biosphere and lithosphere. In this report, the reference unit for the global carbon cycle is $\mathrm{GtC}$ or $\mathrm{GtCO}_{2}$ ( $1 \mathrm{GtC}$ corresponds to $3.667 \mathrm{GtCO}_{2}$ ). Carbon is the major chemical constituent of most organic matter and is stored in the following major reservoirs: organic molecules in the biosphere, carbon dioxide $\left(\mathrm{CO}_{2}\right)$ in the atmosphere, organic matter in the soils, in the lithosphere, and in the oceans.

Carbon dioxide $\left(\mathrm{CO}_{2}\right)$ : A naturally occurring gas, also a by-product of burning fossil fuels from fossil carbon deposits, such as oil, gas and coal, of burning biomass, of land use changes (LUC) and of industrial processes (e.g., cement production). It is the principal anthropogenic greenhouse gas (GHG) that affects the earth's radiative balance. It is the reference gas against which other GHGs are measured and therefore has a Global Warming Potential (GWP) of 1. See Annex II.9.1 for GWP values for other GHGs.

Carbon Dioxide Capture and Storage (CCS): A process in which a relatively pure stream of carbon dioxide $\left(\mathrm{CO}_{2}\right)$ from industrial and energy-related sources is separated (captured), conditioned, compressed, and transported to a storage location for long-term isolation from the atmosphere. See also Bioenergy and carbon capture and storage (BECCS), CCS-ready, and Sequestration.

Carbon dioxide fertilization: The enhancement of the growth of plants as a result of increased atmospheric carbon dioxide $\left(\mathrm{CO}_{2}\right)$ concentration.

Carbon Dioxide Removal (CDR): Carbon Dioxide Removal methods refer to a set of techniques that aim to remove carbon dioxide $\left(\mathrm{CO}_{2}\right)$ directly from the atmosphere by either (1) increasing natural sinks for carbon or (2) using chemical engineering to remove the $\mathrm{CO}_{2}$, with the intent of reducing the atmospheric $\mathrm{CO}_{2}$ concentration. $\mathrm{CDR}$ methods involve the ocean, land, and technical systems, including such methods as iron fertilization, large-scale afforestation, and direct capture of $\mathrm{CO}_{2}$ from the atmosphere using engineered chemical means. Some CDR methods fall under the category of geoengineering, though this may not be the case for others, with the distinction being based on the magnitude, scale, and impact of the particular CDR activities. The 
boundary between CDR and mitigation is not clear and there could be some overlap between the two given current definitions (IPCC, 2012, p. 2). See also Solar Radiation Management (SRM).

Carbon footprint: Measure of the exclusive total amount of emissions of carbon dioxide $\left(\mathrm{CO}_{2}\right)$ that is directly and indirectly caused by an activity or is accumulated over the life stages of a product (Wiedmann and Minx, 2008).

Carbon intensity: The amount of emissions of carbon dioxide $\left(\mathrm{CO}_{2}\right)$ released per unit of another variable such as gross domestic product $(G D P)$, output energy use, or transport.

Carbon leakage: See Leakage.

Carbon pool: See Reservoir.

Carbon price: The price for avoided or released carbon dioxide $\left(\mathrm{CO}_{2}\right)$ or $\mathrm{CO}_{2}$-equivalent emissions. This may refer to the rate of a carbon tax, or the price of emission permits. In many models that are used to assess the economic costs of mitigation, carbon prices are used as a proxy to represent the level of effort in mitigation policies.

Carbon sequestration: See Sequestration.

Carbon tax: A levy on the carbon content of fossil fuels. Because virtually all of the carbon in fossil fuels is ultimately emitted as carbon dioxide $\left(\mathrm{CO}_{2}\right)$, a carbon tax is equivalent to an emission tax on $\mathrm{CO}_{2}$ emissions.

CCS-ready: New large-scale, stationary carbon dioxide $\left(\mathrm{CO}_{2}\right)$ point sources intended to be retrofitted with Carbon Dioxide Capture and Storage (CCS) could be designed and located to be 'CCS-ready' by reserving space for the capture installation, designing the unit for optimal performance when capture is added, and siting the plant to enable access to storage locations. See also Bioenergy and Carbon Dioxide Capture and Storage (BECCS).

Certified Emission Reduction Unit (CER): Equal to one metric tonne of $\mathrm{CO}_{2}$-equivalent emissions reduced or of carbon dioxide $\left(\mathrm{CO}_{2}\right)$ removed from the atmosphere through the Clean Development Mechanism (CDM) (defined in Article 12 of the Kyoto Protocol) project, calculated using Global Warming Potentials (GWP). See also Emissions Reduction Units (ERU) and Emissions trading.

Chemical oxygen demand (COD): The quantity of oxygen required for the complete oxidation of organic chemical compounds in water; used as a measure of the level of organic pollutants in natural and waste waters. See also Biochemical oxygen demand (BOD).

Chlorofluorocarbons (CFCs): A chlorofluorocarbon is an organic compound that contains chlorine, carbon, hydrogen, and fluorine and is used for refrigeration, air conditioning, packaging, plastic foam, insulation, solvents, or aerosol propellants. Because they are not destroyed in the lower atmosphere, CFCs drift into the upper atmosphere where, given suitable conditions, they break down ozone $\left(\mathrm{O}_{3}\right)$. It is one of the greenhouse gases (GHGs) covered under the 1987 Montreal Protocol as a result of which manufacturing of these gases has been phased out and they are being replaced by other compounds, including hydrofluorocarbons (HFCS) which are GHGs covered under the Kyoto Protocol.

Clean Development Mechanism (CDM): A mechanism defined under Article 12 of the Kyoto Protocol through which investors (governments or companies) from developed (Annex B) countries may finance greenhouse gas ( $G H G$ ) emission reduction or removal projects in developing (Non-Annex B) countries, and receive Certified Emission Reduction Units (CERs) for doing so. The CERs can be credited towards the commitments of the respective developed countries. The CDM is intended to facilitate the two objectives of promoting sustainable development (SD) in developing countries and of helping industrialized countries to reach their emissions commitments in a cost-effective way. See also Kyoto Mechanisms.

Climate: Climate in a narrow sense is usually defined as the average weather, or more rigorously, as the statistical description in terms of the mean and variability of relevant quantities over a period of time ranging from months to thousands or millions of years. The classical period for averaging these variables is 30 years, as defined by the World Meteorological Organization. The relevant quantities are most often surface variables such as temperature, precipitation and wind. Climate in a wider sense is the state, including a statistical description, of the climate system.

Climate change: Climate change refers to a change in the state of the climate that can be identified (e.g., by using statistical tests) by changes in the mean and/or the variability of its properties, and that persists for an extended period, typically decades or longer. Climate change may be due to natural internal processes or external forcings such as modulations of the solar cycles, volcanic eruptions and persistent anthropogenic changes in the composition of the atmosphere or in land use. Note that the United Nations Framework Convention on Climate Change (UNFCCC), in its Article 1, defines climate change as: 'a change of climate which is attributed directly or indirectly to human activity that alters the composition of the global atmosphere and which is in addition to natural climate variability observed over comparable time periods'. The UNFCCC thus makes a distinction between climate change attributable to human activities altering the atmospheric composition, and climate variability attributable to natural causes. See also Climate change commitment.

Climate change commitment: Due to the thermal inertia of the ocean and slow processes in the cryosphere and land surfaces, the climate would continue to change even if the atmospheric composition were held fixed at today's values. Past change in atmospheric composition leads to a committed climate change, which continues for 
as long as a radiative imbalance persists and until all components of the climate system have adjusted to a new state. The further change in temperature after the composition of the atmosphere is held constant is referred to as the constant composition temperature commitment or simply committed warming or warming commitment. Climate change commitment includes other future changes, for example in the hydrological cycle, in extreme weather events, in extreme climate events, and in sea level change. The constant emission commitment is the committed climate change that would result from keeping anthropogenic emissions constant and the zero emission commitment is the climate change commitment when emissions are set to zero. See also Climate change.

Climate (change) feedback: An interaction in which a perturbation in one climate quantity causes a change in a second, and the change in the second quantity ultimately leads to an additional change in the first. A negative feedback is one in which the initial perturbation is weakened by the changes it causes; a positive feedback is one in which the initial perturbation is enhanced. In this Assessment Report, a somewhat narrower definition is often used in which the climate quantity that is perturbed is the global mean surface temperature, which in turn causes changes in the global radiation budget. In either case, the initial perturbation can either be externally forced or arise as part of internal variability.

Climate engineering: See Geoengineering.

Climate finance: There is no agreed definition of climate finance. The term 'climate finance' is applied both to the financial resources devoted to addressing climate change globally and to financial flows to developing countries to assist them in addressing climate change. The literature includes several concepts in these categories, among which the most commonly used include:

Incremental costs: The cost of capital of the incremental investment and the change of operating and maintenance costs for a mitigation or adaptation project in comparison to a reference project. It can be calculated as the difference of the net present values of the two projects. See also Additionality.

Incremental investment: The extra capital required for the initial investment for a mitigation or adaptation project in comparison to a reference project. See also Additionality.

Total climate finance: All financial flows whose expected effect is to reduce net greenhouse gas (GHG) emissions and/or to enhance resilience to the impacts of climate variability and the projected climate change. This covers private and public funds, domestic and international flows, expenditures for mitigation and adaptation to current climate variability as well as future climate change.

Total climate finance flowing to developing countries:The amount of the total climate finance invested in developing coun- tries that comes from developed countries. This covers private and public funds.

\section{Private climate finance flowing to developing countries:} Finance and investment by private actors in/from developed countries for mitigation and adaptation activities in developing countries.

Public climate finance flowing to developing countries: Finance provided by developed countries' governments and bilateral institutions as well as by multilateral institutions for mitigation and adaptation activities in developing countries. Most of the funds provided are concessional loans and grants.

Climate model (spectrum or hierarchy): A numerical representation of the climate system based on the physical, chemical and biological properties of its components, their interactions and feedback processes, and accounting for some of its known properties. The climate system can be represented by models of varying complexity, that is, for any one component or combination of components a spectrum or hierarchy of models can be identified, differing in such aspects as the number of spatial dimensions, the extent to which physical, chemical or biological processes are explicitly represented, or the level at which empirical parametrizations are involved. Coupled Atmosphere-Ocean General Circulation Models (AOGCMs) provide a representation of the climate system that is near or at the most comprehensive end of the spectrum currently available. There is an evolution towards more complex models with interactive chemistry and biology. Climate models are applied as a research tool to study and simulate the climate, and for operational purposes, including monthly, seasonal and interannual climate predictions.

Climate prediction: A climate prediction or climate forecast is the result of an attempt to produce (starting from a particular state of the climate system) an estimate of the actual evolution of the climate in the future, for example, at seasonal, interannual, or decadal time scales. Because the future evolution of the climate system may be highly sensitive to initial conditions, such predictions are usually probabilistic in nature. See also Climate projection, and Climate scenario.

Climate projection: A climate projection is the simulated response of the climate system to a scenario of future emission or concentration of greenhouse gases (GHGs) and aerosols, generally derived using climate models. Climate projections are distinguished from climate predictions by their dependence on the emission/concentration/radiative forcing scenario used, which is in turn based on assumptions concerning, for example, future socioeconomic and technological developments that may or may not be realized. See also Climate scenario.

Climate scenario: A plausible and often simplified representation of the future climate, based on an internally consistent set of climatological relationships that has been constructed for explicit use in investigating the potential consequences of anthropogenic climate 
change, often serving as input to impact models. Climate projections often serve as the raw material for constructing climate scenarios, but climate scenarios usually require additional information such as the observed current climate. See also Baseline/reference, Emission scenario, Mitigation scenario, Representative concentration pathways $(R C P S)$, Scenario, Shared socio-economic pathways, Socio-economic scenario, SRES scenarios, Stabilization, and Transformation pathway.

Climate sensitivity: In IPCC reports, equilibrium climate sensitivity (units: ${ }^{\circ} \mathrm{C}$ ) refers to the equilibrium (steady state) change in the annual global mean surface temperature following a doubling of the atmospheric $\mathrm{CO}_{2}$-equivalent concentration. Owing to computational constraints, the equilibrium climate sensitivity in a climate model is sometimes estimated by running an atmospheric general circulation model (GCM) coupled to a mixed-layer ocean model, because equilibrium climate sensitivity is largely determined by atmospheric processes. Efficient models can be run to equilibrium with a dynamic ocean. The climate sensitivity parameter (units: $\left.{ }^{\circ} \mathrm{C}\left(\mathrm{W} \mathrm{m}^{-2}\right)^{-1}\right)$ refers to the equilibrium change in the annual global mean surface temperature following a unit change in radiative forcing.

The effective climate sensitivity (units: ${ }^{\circ} \mathrm{C}$ ) is an estimate of the global mean surface temperature response to doubled carbon dioxide $\left(\mathrm{CO}_{2}\right)$ concentration that is evaluated from model output or observations for evolving non-equilibrium conditions. It is a measure of the strengths of the climate feedbacks at a particular time and may vary with forcing history and climate state, and therefore may differ from equilibrium climate sensitivity.

The transient climate response (units: ${ }^{\circ} \mathrm{C}$ ) is the change in the global mean surface temperature, averaged over a 20-year period, centred at the time of atmospheric $\mathrm{CO}_{2}$ doubling, in a climate model simulation in which $\mathrm{CO}_{2}$ increases at $1 \% \mathrm{yr}^{-1}$. It is a measure of the strength and rapidity of the surface temperature response to greenhouse gas (GHG) forcing.

Climate system: The climate system is the highly complex system consisting of five major components: the atmosphere, the hydrosphere, the cryosphere, the lithosphere and the biosphere, and the interactions between them. The climate system evolves in time under the influence of its own internal dynamics and because of external forcings such as volcanic eruptions, solar variations and anthropogenic forcings such as the changing composition of the atmosphere and land use change (LUC).

Climate threshold: A limit within the climate system that, when crossed, induces a non-linear response to a given forcing. See also Abrupt climate change.

Climate variability: Climate variability refers to variations in the mean state and other statistics (such as standard deviations, the occurrence of extremes, etc.) of the climate on all spatial and temporal scales beyond that of individual weather events. Variability may be due to natural internal processes within the climate system (internal variability), or to variations in natural or anthropogenic external forcing (external variability). See also Climate change.

$\mathrm{CO}_{2}$-equivalent concentration: The concentration of carbon dioxide $\left(\mathrm{CO}_{2}\right)$ that would cause the same radiative forcing as a given mixture of $\mathrm{CO}_{2}$ and other forcing components. Those values may consider only greenhouse gases (GHGs), or a combination of GHGs, aerosols, and surface albedo changes. $\mathrm{CO}_{2}$-equivalent concentration is a metric for comparing radiative forcing of a mix of different forcing components at a particular time but does not imply equivalence of the corresponding climate change responses nor future forcing. There is generally no connection between $\mathrm{CO}_{2}$-equivalent emissions and resulting $\mathrm{CO}_{2}$ equivalent concentrations.

$\mathrm{CO}_{2}$-equivalent emission: The amount of carbon dioxide $\left(\mathrm{CO}_{2}\right)$ emission that would cause the same integrated radiative forcing, over a given time horizon, as an emitted amount of a greenhouse gas (GHG) or a mixture of $\mathrm{GHGs}$. The $\mathrm{CO}_{2}$-equivalent emission is obtained by multiplying the emission of a GHG by its Global Warming Potential (GWP) for the given time horizon (see Annex II.9.1 and WGI AR5 Table 8.A.1 for GWP values of the different GHGs). For a mix of GHGs it is obtained by summing the $\mathrm{CO}_{2}$-equivalent emissions of each gas. $\mathrm{CO}_{2}$-equivalent emission is a common scale for comparing emissions of different GHGs but does not imply equivalence of the corresponding climate change responses. See also $\mathrm{CO}_{2}$-equivalent concentration.

Co-benefits: The positive effects that a policy or measure aimed at one objective might have on other objectives, without yet evaluating the net effect on overall social welfare. Co-benefits are often subject to uncertainty and depend on, among others, local circumstances and implementation practices. Co-benefits are often referred to as ancillary benefits. See also Adverse side-effect, Risk, and Risk tradeoff.

Cogeneration: Cogeneration (also referred to as combined heat and power, or (HP) is the simultaneous generation and useful application of electricity and useful heat.

Combined-cycle gas turbine: A power plant that combines two processes for generating electricity. First, fuel combustion drives a gas turbine. Second, exhaust gases from the turbine are used to heat water to drive a steam turbine.

\section{Combined heat and power (CHP): See Cogeneration.}

\section{Computable General Equilibrium (CGE) Model: See Models.}

Conference of the Parties (COP): The supreme body of the United Nations Framework Convention on Climate Change (UNFCCC), comprising countries with a right to vote that have ratified or acceded to the convention. See also Meeting of the Parties (CMP). 
Confidence: The validity of a finding based on the type, amount, quality, and consistency of evidence (e.g., mechanistic understanding, theory, data, models, expert judgment) and on the degree of agreement. In this report, confidence is expressed qualitatively (Mastrandrea et al., 2010). See WGI AR5 Figure 1.11 for the levels of confidence and WGI AR5 Table 1.2 for the list of likelihood qualifiers. See also Uncertainty.

Consumption-based accounting: Consumption-based accounting provides a measure of emissions released to the atmosphere in order to generate the goods and services consumed by a certain entity (e.g., person, firm, country, or region). See also Production-based accounting.

Contingent valuation method: An approach to quantitatively assess values assigned by people in monetary (willingness to pay) and non-monetary (willingness to contribute with time, resources etc.) terms. It is a direct method to estimate economic values for ecosystem and environmental services. In a survey, people are asked their willingness to pay/contribute for access to, or their willingness to accept compensation for removal of, a specific environmental service, based on a hypothetical scenario and description of the environmental service.

Conventional fuels: See Fossil fuels.

Copenhagen Accord: The political (as opposed to legal) agreement that emerged at the 15th Session of the Conference of the Parties (COP) at which delegates 'agreed to take note' due to a lack of consensus that an agreement would require. Some of the key elements include: recognition of the importance of the scientific view on the need to limit the increase in global mean surface temperature to $2^{\circ}$ C; commitment by Annex / Parties to implement economy-wide emissions targets by 2020 and non-Annex / Parties to implement mitigation actions; agreement to have emission targets of Annex I Parties and their delivery of finance for developing countries subject to Measurement, Reporting and Verification (MRV) and actions by developing countries to be subject to domestic MRV; calls for scaled up financing including a fast track financing of USD 30 billion and USD 100 billion by 2020; the establishment of a new Green Climate Fund (GCF); and the establishment of a new technology mechanism. Some of these elements were later adopted in the Cancún Agreements.

Cost-benefit analysis (CBA): Monetary measurement of all negative and positive impacts associated with a given action. Costs and benefits are compared in terms of their difference and/or ratio as an indicator of how a given investment or other policy effort pays off seen from the society's point of view.

Cost of conserved energy (CCE): See Levelized cost of conserved energy (LCCE).
Cost-effectiveness: A policy is more cost-effective if it achieves a goal, such as a given pollution abatement level, at lower cost. A critical condition for cost-effectiveness is that marginal abatement costs be equal among obliged parties. Integrated models approximate costeffective solutions, unless they are specifically constrained to behave otherwise. Cost-effective mitigation scenarios are those based on a stylized implementation approach in which a single price on carbon dioxide $\left(\mathrm{CO}_{2}\right)$ and other greenhouse gases (GHGs) is applied across the globe in every sector of every country and that rises over time in a way that achieves lowest global discounted costs.

Cost-effectiveness analysis (CEA): A tool based on constrained optimization for comparing policies designed to meet a prespecified target.

Crediting period, Clean Development Mechanism (CDM): The time during which a project activity is able to generate Certified Emission Reduction Units (CERs). Under certain conditions, the crediting period can be renewed up to two times.

Cropland management: The system of practices on land on which agricultural crops are grown and on land that is set aside or temporarily not being used for crop production (UNFCCC, 2002).

Decarbonization: The process by which countries or other entities aim to achieve a low-carbon economy, or by which individuals aim to reduce their carbon consumption.

Decomposition approach: Decomposition methods disaggregate the total amount of historical changes of a policy variable into contributions made by its various determinants.

Deforestation: Conversion of forest to non-forest is one of the major sources of greenhouse gas (GHG) emissions. Under Article 3.3 of the Kyoto Protocol, "the net changes in greenhouse gas emissions by sources and removals by sinks resulting from direct human-induced land-use change and forestry activities, limited to afforestation, reforestation and deforestation since 1990, measured as verifiable changes in carbon stocks in each commitment period, shall be sued to meet the commitments under this Article of each Party included in Annex I". Reducing emissions from deforestation is not eligible for Joint Implementation (JI) or Clean Development Mechanism (CDM) projects but has been introduced in the program of work under REDD (Reducing Emissions from Deforestation and Forest Degradation) under the United Nations Framework Convention on Climate Change (UNFCCC).

For a discussion of the term forest and related terms such as afforestation, reforestation, and deforestation see the IPCC Special Report on Land Use, Land-Use Change and Forestry (IPCC, 2000). See also the report on Definitions and Methodological Options to Inventory Emissions from Direct Human-induced Degradation of Forests and Devegetation of Other Vegetation Types (IPCC, 2003). 
Dematerialization: The ambition to reduce the total material inputs required to deliver a final service.

Descriptive analysis: Descriptive (also termed positive) approaches to analysis focus on how the world works or actors behave, not how they should behave in some idealized world. See also Normative analysis.

Desertification: Land degradation in arid, semi-arid, and dry subhumid areas resulting from various factors, including climatic variations and human activities. Land degradation in arid, semi-arid, and dry sub-humid areas is a reduction or loss of the biological or economic productivity and complexity of rainfed cropland, irrigated cropland, or range, pasture, forest, and woodlands resulting from land uses or from a process or combination of processes, including processes arising from human activities and habitation patterns, such as (1) soil erosion caused by wind and/or water; (2) deterioration of the physical, chemical, biological, or economic properties of soil; and (3) long-term loss of natural vegetation (UNCCD, 1994).

Designated national authority (DNA): A designated national authority is a national institution that authorizes and approves Clean Development Mechansim (CDM) projects in that country. In CDM host countries, the DNA assesses whether proposed projects assist the host country in achieving its sustainable development (SD) goals, certification of which is a prerequisite for registration of the project by the CDM Executive Board.

Developed/developing countries: See Industrialized/developing countries.

Development pathway: An evolution based on an array of technological, economic, social, institutional, cultural, and biophysical characteristics that determine the interactions between human and natural systems, including consumption and production patterns in all countries, over time at a particular scale.

Direct Air Capture (DAC): Chemical process by which a pure carbon dioxide $\left(\mathrm{CO}_{2}\right)$ stream is produced by capturing $\mathrm{CO}_{2}$ from the ambient air.

\section{Direct emissions: See Emissions.}

Discounting: A mathematical operation making monetary (or other) amounts received or expended at different times (years) comparable across time. The discounter uses a fixed or possibly time-varying discount rate $(>0)$ from year to year that makes future value worth less today. See also Present value.

Double dividend: The extent to which revenue-generating instruments, such as carbon taxes or auctioned (tradable) emission permits can (1) contribute to mitigation and (2) offset at least part of the potential welfare losses of climate policies through recycling the revenue in the economy to reduce other taxes likely to cause distortions.
Drivers of behaviour: Determinants of human decisions and actions, including peoples' values and goals and the factors that constrain action, including economic factors and incentives, information access, regulatory and technological constraints, cognitive and emotional processing capacity, and social norms. See also Behaviour and Behavioural change.

Drivers of emissions: Drivers of emissions refer to the processes, mechanisms and properties that influence emissions through factors. Factors comprise the terms in a decomposition of emissions. Factors and drivers may in return affect policies, measures and other drivers.

Economic efficiency: Economic efficiency refers to an economy's allocation of resources (goods, services, inputs, productive activities). An allocation is efficient if it is not possible to reallocate resources so as to make at least one person better off without making someone else worse off. An allocation is inefficient if such a reallocation is possible. This is also known as the Pareto Criterion for efficiency. See also Pareto optimum.

Economies in Transition (EITs): Countries with their economies changing from a planned economic system to a market economy. See Annex II.2.1.

Ecosystem: A functional unit consisting of living organisms, their nonliving environment, and the interactions within and between them. The components included in a given ecosystem and its spatial boundaries depend on the purpose for which the ecosystem is defined: in some cases they are relatively sharp, while in others they are diffuse. Ecosystem boundaries can change over time. Ecosystems are nested within other ecosystems, and their scale can range from very small to the entire biosphere. In the current era, most ecosystems either contain people as key organisms, or are influenced by the effects of human activities in their environment.

Ecosystem services: Ecological processes or functions having monetary or non-monetary value to individuals or society at large. These are frequently classified as (1) supporting services such as productivity or biodiversity maintenance, (2) provisioning services such as food, fiber, or fish, (3) regulating services such as climate regulation or carbon sequestration, and (4) cultural services such as tourism or spiritual and aesthetic appreciation.

\section{Embodied emissions: See Emissions.}

Embodied energy: See Energy.

Emission allowance: See Emission permit.

Emission factor/Emissions intensity: The emissions released per unit of activity. See also Carbon intensity. 
Emission permit: An entitlement allocated by a government to a legal entity (company or other emitter) to emit a specified amount of a substance. Emission permits are often used as part of emissions trading schemes.

Emission quota: The portion of total allowable emissions assigned to a country or group of countries within a framework of maximum total emissions.

Emission scenario: A plausible representation of the future development of emissions of substances that are potentially radiatively active (e.g., greenhouse gases, aerosols) based on a coherent and internally consistent set of assumptions about driving forces (such as demographic and socioeconomic development, technological change, energy and land use) and their key relationships. Concentration scenarios, derived from emission scenarios, are used as input to a climate model to compute climate projections. In IPCC (1992) a set of emission scenarios was presented which were used as a basis for the climate projections in IPCC (1996). These emission scenarios are referred to as the IS92 scenarios. In the IPCC Special Report on Emission Scenarios (Nakićenović and Swart, 2000) emission scenarios, the so-called SRES scenarios, were published, some of which were used, among others, as a basis for the climate projections presented in Chapters 9 to 11 of IPCC (2001) and Chapters 10 and 11 of IPCC (2007). New emission scenarios for climate change, the four Representative Concentration Pathways (RCPS), were developed for, but independently of, the present IPCC assessment. See also Baseline/reference, Climate scenario, Mitigation scenario, Shared socio-economic pathways, Scenario, Socio-economic scenario, Stabilization, and Transformation pathway.

Emission trajectories: A projected development in time of the emission of a greenhouse gas (GHG) or group of GHGs, aerosols, and GHG precursors.

Emissions:

Agricultural emissions: Emissions associated with agricultural systems-predominantly methane $\left(\mathrm{CH}_{4}\right)$ or nitrous oxide $\left(\mathrm{N}_{2} \mathrm{O}\right)$. These include emissions from enteric fermentation in domestic livestock, manure management, rice cultivation, prescribed burning of savannas and grassland, and from soils (IPCC, 2006).

Anthropogenic emissions: Emissions of greenhouse gases (GHGS), aerosols, and precursors of a GHG or aerosol caused by human activities. These activities include the burning of fossil fuels, deforestation, land use changes (LUC), livestock production, fertilization, waste management, and industrial processes.

Direct emissions: Emissions that physically arise from activities within well-defined boundaries of, for instance, a region, an economic sector, a company, or a process.
Embodied emissions: Emissions that arise from the production and delivery of a good or service or the build-up of infrastructure. Depending on the chosen system boundaries, upstream emissions are often included (e.g., emissions resulting from the extraction of raw materials). See also Lifecycle assessment (LCA).

Indirect emissions: Emissions that are a consequence of the activities within well-defined boundaries of, for instance, a region, an economic sector, a company or process, but which occur outside the specified boundaries. For example, emissions are described as indirect if they relate to the use of heat but physically arise outside the boundaries of the heat user, or to electricity production but physically arise outside of the boundaries of the power supply sector.

Scope 1, Scope 2, and Scope 3 emissions: Emissions responsibility as defined by the GHG Protocol, a private sector initiative. 'Scope 1' indicates direct greenhouse gas (GHG) emissions that are from sources owned or controlled by the reporting entity. 'Scope 2 ' indicates indirect GHG emissions associated with the production of electricity, heat, or steam purchased by the reporting entity. 'Scope 3' indicates all other indirect emissions, i. e., emissions associated with the extraction and production of purchased materials, fuels, and services, including transport in vehicles not owned or controlled by the reporting entity, outsourced activities, waste disposal, etc. (WBCSD and WRI, 2004).

Territorial emissions: Emissions that take place within the territories of a particular jurisdiction.

Emissions Reduction Unit (ERU): Equal to one metric tonne of $\mathrm{CO}_{2}$ equivalent emissions reduced or of carbon dioxide $\left(\mathrm{CO}_{2}\right)$ removed from the atmosphere through a Joint Implementation (JI) (defined in Article 6 of the Kyoto Protocol) project, calculated using Global Warming Potentials (GWPS). See also Certified Emission Reduction Unit (CER) and Emissions trading.

Emission standard: An emission level that, by law or by voluntary agreement, may not be exceeded. Many standards use emission factors in their prescription and therefore do not impose absolute limits on the emissions.

Emissions trading: A market-based instrument used to limit emissions. The environmental objective or sum of total allowed emissions is expressed as an emissions cap. The cap is divided in tradable emission permits that are allocated-either by auctioning or handing out for free (grandfathering) - to entities within the jurisdiction of the trading scheme. Entities need to surrender emission permits equal to the amount of their emissions (e.g., tonnes of carbon dioxide). An entity may sell excess permits. Trading schemes may occur at the intra-company, domestic, or international level and may apply to carbon dioxide $\left(\mathrm{CO}_{2}\right)$, other greenhouse gases (GHGs), or other substances. Emissions 
trading is also one of the mechanisms under the Kyoto Protocol. See also Kyoto Mechanisms.

Energy: The power of 'doing work' possessed at any instant by a body or system of bodies. Energy is classified in a variety of types and becomes available to human ends when it flows from one place to another or is converted from one type into another.

Embodied energy: The energy used to produce a material substance or product (such as processed metals or building materials), taking into account energy used at the manufacturing facility, energy used in producing the materials that are used in the manufacturing facility, and so on.

\section{Final energy: See Primary energy.}

Primary energy: Primary energy (also referred to as energy sources) is the energy stored in natural resources (e. g., coal, crude oil, natural gas, uranium, and renewable sources). It is defined in several alternative ways. The International Energy Agency (IEA) utilizes the physical energy content method, which defines primary energy as energy that has not undergone any anthropogenic conversion. The method used in this report is the direct equivalent method (see Annex II.4), which counts one unit of secondary energy provided from non-combustible sources as one unit of primary energy, but treats combustion energy as the energy potential contained in fuels prior to treatment or combustion. Primary energy is transformed into secondary energy by cleaning (natural gas), refining (crude oil to oil products) or by conversion into electricity or heat. When the secondary energy is delivered at the enduse facilities it is called final energy (e.g., electricity at the wall outlet), where it becomes usable energy in supplying energy services (e. g., light).

Renewable energy (RE): Any form of energy from solar, geophysical, or biological sources that is replenished by natural processes at a rate that equals or exceeds its rate of use. For a more detailed description see Bioenergy, Solar energy, Hydropower, Ocean, Geothermal, and Wind energy.

Secondary energy: See Primary energy.

Energy access: Access to clean, reliable and affordable energy services for cooking and heating, lighting, communications, and productive uses (AGECC, 2010).

Energy carrier: A substance for delivering mechanical work or transfer of heat. Examples of energy carriers include: solid, liquid, or gaseous fuels (e.g., biomass, coal, oil, natural gas, hydrogen); pressur$\mathrm{ized/heated/cooled} \mathrm{fluids} \mathrm{(air,} \mathrm{water,} \mathrm{steam);} \mathrm{and} \mathrm{electric} \mathrm{current.}$

Energy density: The ratio of stored energy to the volume or mass of a fuel or battery.
Energy efficiency (EE): The ratio of useful energy output of a system, conversion process, or activity to its energy input. In economics, the term may describe the ratio of economic output to energy input. See also Energy intensity.

Energy intensity: The ratio of energy use to economic or physical output.

Energy poverty: A lack of access to modern energy services. See also Energy access.

Energy security: The goal of a given country, or the global community as a whole, to maintain an adequate, stable, and predictable energy supply. Measures encompass safeguarding the sufficiency of energy resources to meet national energy demand at competitive and stable prices and the resilience of the energy supply; enabling development and deployment of technologies; building sufficient infrastructure to generate, store and transmit energy supplies; and ensuring enforceable contracts of delivery.

Energy services: An energy service is the benefit received as a result of energy use.

Energy system: The energy system comprises all components related to the production, conversion, delivery, and use of energy.

Environmental effectiveness: A policy is environmentally effective to the extent by which it achieves its expected environmental target (e. g., greenhouse gas (GHG) emission reduction).

Environmental input-output analysis: An analytical method used to allocate environmental impacts arising in production to categories of final consumption, by means of the Leontief inverse of a country's economic input-output tables. See also Annex II.6.2.

Environmental Kuznets Curve: The hypothesis that various environmental impacts first increase and then eventually decrease as income per capita increases.

Evidence: Information indicating the degree to which a belief or proposition is true or valid. In this report, the degree of evidence reflects the amount, quality, and consistency of scientific/technical information on which the Lead Authors are basing their findings. See also Agreement, Confidence, Likelihood and Uncertainty.

Externality/external cost/external benefit: Externalities arise from a human activity when agents responsible for the activity do not take full account of the activity's impacts on others' production and consumption possibilities, and no compensation exists for such impacts. When the impacts are negative, they are external costs. When the impacts are positive, they are external benefits. See also Social costs. 
Feed-in tariff (FIT): The price per unit of electricity (heat) that a utility or power (heat) supplier has to pay for distributed or renewable electricity (heat) fed into the power grid (heat supply system) by non-utility generators. A public authority regulates the tariff.

Final energy: See Primary energy.

Flaring: Open air burning of waste gases and volatile liquids, through a chimney, at oil wells or rigs, in refineries or chemical plants, and at landfills.

\section{Flexibility Mechanisms: See Kyoto Mechanisms.}

Food security: A state that prevails when people have secure access to sufficient amounts of safe and nutritious food for normal growth, development, and an active and healthy life. ${ }^{4}$

Forest: A vegetation type dominated by trees. Many definitions of the term forest are in use throughout the world, reflecting wide differences in biogeophysical conditions, social structure and economics. According to the 2005 United Nations Framework Convention on Climate Change (UNFCCC) definition a forest is an area of land of at least $0.05-1$ hectare, of which more than $10-30 \%$ is covered by tree canopy. Trees must have a potential to reach a minimum of 25 meters at maturity in situ. Parties to the Convention can choose to define a forest from within those ranges. Currently, the definition does not recognize different biomes, nor do they distinguish natural forests from plantations, an anomaly being pointed out by many as in need of rectification.

For a discussion of the term forest and related terms such as afforestation, reforestation and deforestation see the IPCC Report on Land Use, Land-Use Change and Forestry (IPCC, 2000). See also the Report on Definitions and Methodological Options to Inventory Emissions from Direct Human-induced Degradation of Forests and Devegetation of Other Vegetation Types (IPCC, 2003).

Forest management: A system of practices for stewardship and use of forest land aimed at fulfilling relevant ecological (including biological diversity), economic and social functions of the forest in a sustainable manner (UNFCCC, 2002).

Forestry and Other Land Use (FOLU): See Agriculture, Forestry and Other Land Use (AFOLU).

Fossil fuels: Carbon-based fuels from fossil hydrocarbon deposits, including coal, peat, oil, and natural gas.

Free Rider: One who benefits from a common good without contributing to its creation or preservation.

4 This glossary entry builds on definitions used in FAO (2000) and previous IPCC reports.
Fuel cell: A fuel cell generates electricity in a direct and continuous way from the controlled electrochemical reaction of hydrogen or another fuel and oxygen. With hydrogen as fuel the cell emits only water and heat (no carbon dioxide) and the heat can be utilized (see also Cogeneration).

Fuel poverty: A condition in which a household is unable to guarantee a certain level of consumption of domestic energy services (especially heating) or suffers disproportionate expenditure burdens to meet these needs.

Fuel switching: In general, fuel switching refers to substituting fuel A for fuel $B$. In the context of mitigation it is implicit that fuel $A$ has lower carbon content than fuel B, e. g., switching from natural gas to coal.

\section{General circulation (climate) model (GCM): See Climate model.}

General equilibrium analysis: General equilibrium analysis considers simultaneously all the markets and feedback effects among these markets in an economy leading to market clearance. (Computable) general equilibrium (CGE) models are the operational tools used to perform this type of analysis.

Geoengineering: Geoengineering refers to a broad set of methods and technologies that aim to deliberately alter the climate system in order to alleviate the impacts of climate change. Most, but not all, methods seek to either (1) reduce the amount of absorbed solar energy in the climate system (Solar Radiation Management) or (2) increase net carbon sinks from the atmosphere at a scale sufficiently large to alter climate (Carbon Dioxide Removal). Scale and intent are of central importance. Two key characteristics of geoengineering methods of particular concern are that they use or affect the climate system (e. g., atmosphere, land or ocean) globally or regionally and/or could have substantive unintended effects that cross national boundaries. Geoengineering is different from weather modification and ecological engineering, but the boundary can be fuzzy (IPCC, 2012, p. 2).

Geothermal energy: Accessible thermal energy stored in the earth's interior.

Global Environment Facility (GEF): The Global Environment Facility, established in 1991, helps developing countries fund projects and programmes that protect the global environment. GEF grants support projects related to biodiversity, climate change, international waters, land degradation, the ozone $\left(\mathrm{O}_{3}\right)$ layer, and persistent organic pollutants.

Global mean surface temperature: An estimate of the global mean surface air temperature. However, for changes over time, only anomalies, as departures from a climatology, are used, most commonly based on the area-weighted global average of the sea surface temperature anomaly and land surface air temperature anomaly. 
Global warming: Global warming refers to the gradual increase, observed or projected, in global surface temperature, as one of the consequences of radiative forcing caused by anthropogenic emissions.

Global Warming Potential (GWP): An index, based on radiative properties of greenhouse gases (GHGs), measuring the radiative forcing following a pulse emission of a unit mass of a given GHG in the present-day atmosphere integrated over a chosen time horizon, relative to that of carbon dioxide $\left(\mathrm{CO}_{2}\right)$. The GWP represents the combined effect of the differing times these gases remain in the atmosphere and their relative effectiveness in causing radiative forcing. The Kyoto Protocol is based on GWPs from pulse emissions over a 100-year time frame. Unless stated otherwise, this report uses GWP values calculated with a 100-year time horizon which are often derived from the IPCC Second Assessment Report (see Annex II.9.1 for the GWP values of the different GHGs).

Governance: A comprehensive and inclusive concept of the full range of means for deciding, managing, and implementing policies and measures. Whereas government is defined strictly in terms of the nationstate, the more inclusive concept of governance recognizes the contributions of various levels of government (global, international, regional, local) and the contributing roles of the private sector, of nongovernmental actors, and of civil society to addressing the many types of issues facing the global community.

Grazing land management: The system of practices on land used for livestock production aimed at manipulating the amount and type of vegetation and livestock produced (UNFCCC, 2002).

Green Climate Fund (GCF): The Green Climate Fund was established by the 16th Session of the Conference of the Parties (COP) in 2010 as an operating entity of the financial mechanism of the United Nations Framework Convention on Climate Change (UNFCCC), in accordance with Article 11 of the Convention, to support projects, programmes and policies and other activities in developing country Parties. The Fund is governed by a Board and will receive guidance of the COP. The Fund is headquartered in Songdo, Republic of Korea.

Greenhouse effect: The infrared radiative effect of all infraredabsorbing constituents in the atmosphere. Greenhouse gases (GHGS), clouds, and (to a small extent) aerosols absorb terrestrial radiation emitted by the earth's surface and elsewhere in the atmosphere. These substances emit infrared radiation in all directions, but, everything else being equal, the net amount emitted to space is normally less than would have been emitted in the absence of these absorbers because of the decline of temperature with altitude in the troposphere and the consequent weakening of emission. An increase in the concentration of GHGs increases the magnitude of this effect; the difference is sometimes called the enhanced greenhouse effect. The change in a GHG concentration because of anthropogenic emissions contributes to an instantaneous radiative forcing. Surface temperature and troposphere warm in response to this forcing, gradually restoring the radiative balance at the top of the atmosphere.

Greenhouse gas (GHG): Greenhouse gases are those gaseous constituents of the atmosphere, both natural and anthropogenic, that absorb and emit radiation at specific wavelengths within the spectrum of terrestrial radiation emitted by the earth's surface, the atmosphere itself, and by clouds. This property causes the greenhouse effect. Water vapour $\left(\mathrm{H}_{2} \mathrm{O}\right)$, carbon dioxide $\left(\mathrm{CO}_{2}\right)$, nitrous oxide $\left(\mathrm{N}_{2} \mathrm{O}\right)$, methane $\left(\mathrm{CH}_{4}\right)$ and ozone $\left(\mathrm{O}_{3}\right)$ are the primary GHGs in the earth's atmosphere. Moreover, there are a number of entirely human-made GHGs in the atmosphere, such as the halocarbons and other chlorine- and brominecontaining substances, dealt with under the Montreal Protocol. Beside $\mathrm{CO}_{2}, \mathrm{~N}_{2} \mathrm{O}$ and $\mathrm{CH}_{4}$, the Kyoto Protocol deals with the GHGs sulphur hexafluoride (SF), hydrofluorocarbons (HFCS) and perfluorocarbons (PFCS). For a list of well-mixed GHGs, see WGI AR5 Table 2.A.1.

Gross domestic product (GDP): The sum of gross value added, at purchasers' prices, by all resident and non-resident producers in the economy, plus any taxes and minus any subsidies not included in the value of the products in a country or a geographic region for a given period, normally one year. GDP is calculated without deducting for depreciation of fabricated assets or depletion and degradation of natural resources.

Gross national expenditure (GNE): The total amount of public and private consumption and capital expenditures of a nation. In general, national account is balanced such that gross domestic product (GDP) + import $=\mathrm{GNE}+$ export.

Gross national product: The value added from domestic and foreign sources claimed by residents. GNP comprises gross domestic product (GDP) plus net receipts of primary income from non-resident income.

Gross world product: An aggregation of the individual country's gross domestic products (GDP) to obtain the world or global GDP.

Heat island: The relative warmth of a city compared with surrounding rural areas, associated with changes in runoff, effects on heat retention, and changes in surface albedo.

Human Development Index (HDI): The Human Development Index allows the assessment of countries' progress regarding social and economic development as a composite index of three indicators: (1) health measured by life expectancy at birth; (2) knowledge as measured by a combination of the adult literacy rate and the combined primary, secondary and tertiary school enrolment ratio; and (3) standard of living as gross domestic product (GDP) per capita (in purchasing power parity). The HDI sets a minimum and a maximum for each dimension, called goalposts, and then shows where each country stands in relation to these goalposts, expressed as a value between 0 and 1 . The HDI only acts as a broad proxy for some of the key issues of human 
development; for instance, it does not reflect issues such as political participation or gender inequalities.

Hybrid vehicle: Any vehicle that employs two sources of propulsion, particularly a vehicle that combines an internal combustion engine with an electric motor.

Hydrofluorocarbons (HFCs): One of the six types of greenhouse gases (GHGs) or groups of GHGs to be mitigated under the Kyoto Protocol. They are produced commercially as a substitute for chlorofluorocarbons (CFCS). HFCs largely are used in refrigeration and semiconductor manufacturing. See also Global Warming Potential (GWP) and Annex II.9.1 for GWP values.

Hydropower: Power harnessed from the flow of water.

Incremental costs: See Climate finance.

Incremental investment: See Climate finance.

Indigenous peoples: Indigenous peoples and nations are those that, having a historical continuity with pre-invasion and pre-colonial societies that developed on their territories, consider themselves distinct from other sectors of the societies now prevailing on those territories, or parts of them. They form at present principally non-dominant sectors of society and are often determined to preserve, develop, and transmit to future generations their ancestral territories, and their ethnic identity, as the basis of their continued existence as peoples, in accordance with their own cultural patterns, social institutions, and common law system. ${ }^{5}$

\section{Indirect emissions: See Emissions.}

Indirect land use change (iLUC): See Land use.

Industrial Revolution: A period of rapid industrial growth with farreaching social and economic consequences, beginning in Britain during the second half of the 18th century and spreading to Europe and later to other countries including the United States. The invention of the steam engine was an important trigger of this development. The industrial revolution marks the beginning of a strong increase in the use of fossil fuels and emission of, in particular, fossil carbon dioxide. In this report the terms pre-industrial and industrial refer, somewhat arbitrarily, to the periods before and after 1750 , respectively.

Industrialized countries/developing countries: There are a diversity of approaches for categorizing countries on the basis of their level of development, and for defining terms such as industrialized, developed, or developing. Several categorizations are used in this report. (1)

This glossary entry builds on the definitions used in Cobo (1987) and previous IPCC reports.
In the United Nations system, there is no established convention for designating of developed and developing countries or areas. (2) The United Nations Statistics Division specifies developed and developing regions based on common practice. In addition, specific countries are designated as Least Developed Countries (LCD), landlocked developing countries, small island developing states, and transition economies. Many countries appear in more than one of these categories. (3) The World Bank uses income as the main criterion for classifying countries as low, lower middle, upper middle, and high income. (4) The UNDP aggregates indicators for life expectancy, educational attainment, and income into a single composite Human Development Index (HDI) to classify countries as low, medium, high, or very high human development. See WGII AR5 Box 1-2.

Input-output analysis: See Environmental input-output analysis.

Institution: Institutions are rules and norms held in common by social actors that guide, constrain and shape human interaction. Institutions can be formal, such as laws and policies, or informal, such as norms and conventions. Organizations-such as parliaments, regulatory agencies, private firms, and community bodies-develop and act in response to institutional frameworks and the incentives they frame. Institutions can guide, constrain and shape human interaction through direct control, through incentives, and through processes of socialization.

Institutional feasibility: Institutional feasibility has two key parts: (1) the extent of administrative workload, both for public authorities and for regulated entities, and (2) the extent to which the policy is viewed as legitimate, gains acceptance, is adopted, and is implemented.

Integrated assessment: A method of analysis that combines results and models from the physical, biological, economic, and social sciences, and the interactions among these components in a consistent framework to evaluate the status and the consequences of environmental change and the policy responses to it. See also Integrated Models.

Integrated models: See Models.

IPAT identity: IPAT is the lettering of a formula put forward to describe the impact of human activity on the environment. Impact (I) is viewed as the product of population size $(P)$, affluence $(A=G D P /$ person) and technology ( $T=$ impact per GDP unit). In this conceptualization, population growth by definition leads to greater environmental impact if $A$ and $T$ are constant, and likewise higher income leads to more impact (Ehrlich and Holdren, 1971).

Iron fertilization: Deliberate introduction of iron to the upper ocean intended to enhance biological productivity which can sequester additional atmospheric carbon dioxide $\left(\mathrm{CO}_{2}\right)$ into the oceans. See also Geoengineering and Carbon Dioxide Removal (CDR).

Jevon's paradox: See Rebound effect. 
Joint Implementation (J): A mechanism defined in Article 6 of the Kyoto Protocol, through which investors (governments or companies) from developed (Annex B) countries may implement projects jointly that limit or reduce emissions or enhance sinks, and to share the Emissions Reduction Units (ERU). See also Kyoto Mechanisms.

Kaya identity: In this identity global emissions are equal to the population size, multiplied by per capita output (gross world product), multiplied by the energy intensity of production, multiplied by the carbon intensity of energy.

Kyoto Mechanisms (also referred to as Flexibility Mechanisms): Market-based mechanisms that Parties to the Kyoto Protoco/ can use in an attempt to lessen the potential economic impacts of their commitment to limit or reduce greenhouse gas (GHG) emissions. They include Joint Implementation (JI) (Article 6), Clean Development Mechanism (CDM) (Article 12), and Emissions trading (Article 17).

Kyoto Protocol: The Kyoto Protocol to the United Nations Framework Convention on Climate Change (UNFCCC) was adopted in 1997 in Kyoto, Japan, at the Third Session of the Conference of the Parties (COP) to the UNFCCC. It contains legally binding commitments, in addition to those included in the UNFCCC. Countries included in Annex $B$ of the Protocol (most Organisation for Economic Cooperation and Development countries and countries with economies in transition) agreed to reduce their anthropogenic greenhouse gas (GHG) emissions (carbon dioxide $\left(\mathrm{CO}_{2}\right)$, methane $\left(\mathrm{CH}_{4}\right)$, nitrous oxide $\left(\mathrm{N}_{2} \mathrm{O}\right)$, hydrofluorocarbons (HFCS), perfluorocarbons (PFCS), and sulphur hexafluoride $\left(S F_{6}\right)$ ) by at least 5\% below 1990 levels in the commitment period 2008-2012. The Kyoto Protocol entered into force on 16 February 2005.

Land use (change, direct and indirect): Land use refers to the total of arrangements, activities and inputs undertaken in a certain land cover type (a set of human actions). The term land use is also used in the sense of the social and economic purposes for which land is managed (e.g., grazing, timber extraction and conservation). In urban settlements it is related to land uses within cities and their hinterlands. Urban land use has implications on city management, structure, and form and thus on energy demand, greenhouse gas (GHG) emissions, and mobility, among other aspects.

Land use change (LUC): Land use change refers to a change in the use or management of land by humans, which may lead to a change in land cover. Land cover and LUC may have an impact on the surface albedo, evapotranspiration, sources and sinks of GHGs, or other properties of the climate system and may thus give rise to radiative forcing and/or other impacts on climate, locally or globally. See also the IPCC Report on Land Use, Land-Use Change, and Forestry (IPCC, 2000).

Indirect land use change (iLUC): Indirect land use change refers to shifts in land use induced by a change in the production level of an agricultural product elsewhere, often mediated by markets or driven by policies. For example, if agricultural land is diverted to fuel production, forest clearance may occur elsewhere to replace the former agricultural production. See also Afforestation, Deforestation and Reforestation.

Land use, land use change and forestry (LULUCF): A greenhouse gas (GHG) inventory sector that covers emissions and removals of GHGs resulting from direct human-induced land use, land use change and forestry activities excluding agricultural emissions. See also Agriculture, Forestry and Other Land Use (AFOLU).

Land value capture: A financing mechanism usually based around transit systems, or other infrastructure and services, that captures the increased value of land due to improved accessibility.

Leakage: Phenomena whereby the reduction in emissions (relative to a baseline) in a jurisdiction/sector associated with the implementation of mitigation policy is offset to some degree by an increase outside the jurisdiction/sector through induced changes in consumption, production, prices, land use and/or trade across the jurisdictions/sectors. Leakage can occur at a number of levels, be it a project, state, province, nation, or world region. See also Rebound effect.

In the context of Carbon Dioxide Capture and Storage (CCS), 'CO leakage' refers to the escape of injected carbon dioxide $\left(\mathrm{CO}_{2}\right)$ from the storage location and eventual release to the atmosphere. In the context of other substances, the term is used more generically, such as for 'methane ( $\mathrm{CH}_{4}$ ) leakage' (e.g., from fossil fuel extraction activities), and 'hydrofluorocarbon (HFC) leakage' (e.g., from refrigeration and air-conditioning systems).

Learning curve/rate: Decreasing cost-prices of technologies shown as a function of increasing (total or yearly) supplies. The learning rate is the percent decrease of the cost-price for every doubling of the cumulative supplies (also called progress ratio).

Least Developed Countries (LDCs): A list of countries designated by the Economic and Social Council of the United Nations (ECOSOC) as meeting three criteria: (1) a low income criterion below a certain threshold of gross national income per capita of between USD 750 and USD 900, (2) a human resource weakness based on indicators of health, education, adult literacy, and (3) an economic vulnerability weakness based on indicators on instability of agricultural production, instability of export of goods and services, economic importance of non-traditional activities, merchandise export concentration, and the handicap of economic smallness. Countries in this category are eligible for a number of programmes focused on assisting countries most in need. These privileges include certain benefits under the articles of the United Nations Framework Convention on Climate Change (UNFCCC). See also Industrialized/developing countries.

Levelized cost of conserved carbon (LCCC): See Annex II.3.1.3 for concepts and definition. 
Levelized cost of conserved energy (LCCE): See Annex II.3.1.2 for concepts and definition.

Levelized cost of energy (LCOE): See Annex II.3.1.1 for concepts and definition.

Lifecycle assessment (LCA): A widely used technique defined by ISO 14040 as a "compilation and evaluation of the inputs, outputs and the potential environmental impacts of a product system throughout its life cycle". The results of LCA studies are strongly dependent on the system boundaries within which they are conducted. The technique is intended for relative comparison of two similar means to complete a product. See also Annex II.6.3.

Likelihood: The chance of a specific outcome occurring, where this might be estimated probabilistically. This is expressed in this report using a standard terminology (Mastrandrea et al., 2010): virtually certain $99-100 \%$ probability, very likely $90-100 \%$, likely $66-100 \%$, about as likely as not 33-66 \%, unlikely $0-33 \%$, very unlikely $0-10$ $\%$, exceptionally unlikely $0-1 \%$. Additional terms (more likely than not $>50-100 \%$, and more unlikely than likely $0-<50 \%$ ) may also be used when appropriate. Assessed likelihood is typeset in italics, e. g., very likely. See also Agreement, Confidence, Evidence and Uncertainty.

Lock-in: Lock-in occurs when a market is stuck with a standard even though participants would be better off with an alternative.

Marginal abatement cost (MAC): The cost of one unit of additional mitigation.

Market barriers: In the context of climate change mitigation, market barriers are conditions that prevent or impede the diffusion of costeffective technologies or practices that would mitigate greenhouse gas (GHG) emissions.

Market-based mechanisms, GHG emissions: Regulatory approaches using price mechanisms (e.g., taxes and auctioned emission permits), among other instruments, to reduce the sources or enhance the sinks of greenhouse gases (GHGs).

Market exchange rate (MER): The rate at which foreign currencies are exchanged. Most economies post such rates daily and they vary little across all the exchanges. For some developing economies, official rates and black-market rates may differ significantly and the MER is difficult to pin down. See also Purchasing power parity (PPP) and Annex II.1.3 for the monetary conversion process applied throughout this report.

Market failure: When private decisions are based on market prices that do not reflect the real scarcity of goods and services but rather reflect market distortions, they do not generate an efficient allocation of resources but cause welfare losses. A market distortion is any event in which a market reaches a market clearing price that is substantially different from the price that a market would achieve while operating under conditions of perfect competition and state enforcement of legal contracts and the ownership of private property. Examples of factors causing market prices to deviate from real economic scarcity are environmental externalities, public goods, monopoly power, information asymmetry, transaction costs, and non-rational behaviour. See also Economic efficiency.

Material flow analysis (MFA): A systematic assessment of the flows and stocks of materials within a system defined in space and time (Brunner and Rechberger, 2004). See also Annex II.6.1.

Measures: In climate policy, measures are technologies, processes or practices that contribute to mitigation, for example renewable energy (RE) technologies, waste minimization processes, public transport commuting practices.

Meeting of the Parties (CMP): The Conference of the Parties (COP) to the United Nations Framework Convention on Climate Change (UNFCCC) serves as the CMP, the supreme body of the Kyoto Protocol, since the latter entered into force on 16 February 2005. Only Parties to the Kyoto Protocol may participate in deliberations and make decisions.

Methane $\left(\mathrm{CH}_{4}\right)$ : One of the six greenhouse gases (GHGs) to be mitigated under the Kyoto Protocol and is the major component of natural gas and associated with all hydrocarbon fuels. Significant emissions occur as a result of animal husbandry and agriculture and their management represents a major mitigation option. See also Global Warming Potential (GWP) and Annex II.9.1 for GWP values.

Methane recovery: Any process by which methane $\left(\mathrm{CH}_{4}\right)$ emissions (e. g., from oil or gas wells, coal beds, peat bogs, gas transmission pipelines, landfills, or anaerobic digesters) are captured and used as a fuel or for some other economic purpose (e. g., chemical feedstock).

Millennium Development Goals (MDGs): A set of eight time-bound and measurable goals for combating poverty, hunger, disease, illiteracy, discrimination against women and environmental degradation. These goals were agreed to at the UN Millennium Summit in 2000 together with an action plan to reach the goals.

Mitigation (of climate change): A human intervention to reduce the sources or enhance the sinks of greenhouse gases (GHGs). This report also assesses human interventions to reduce the sources of other substances which may contribute directly or indirectly to limiting climate change, including, for example, the reduction of particulate matter (PM) emissions that can directly alter the radiation balance (e.g., black carbon) or measures that control emissions of carbon monoxide, nitrogen oxides $\left(\mathrm{NO}_{x}\right)$, Volatile Organic Compounds (VOCS) and other 
pollutants that can alter the concentration of tropospheric ozone $\left(\mathrm{O}_{3}\right)$ which has an indirect effect on the climate.

Mitigation capacity: A country's ability to reduce anthropogenic greenhouse gas (GHG) emissions or to enhance natural sinks, where ability refers to skills, competencies, fitness, and proficiencies that a country has attained and depends on technology, institutions, wealth, equity, infrastructure, and information. Mitigative capacity is rooted in a country's sustainable development (SD) path.

Mitigation scenario: A plausible description of the future that describes how the (studied) system responds to the implementation of mitigation policies and measures. See also Baseline/reference, Climate scenario, Emission scenario, Representative Concentration Pathways (RCPS), Scenario, Shared socio-economic pathways, Socioeconomic scenarios, SRES scenarios, Stabilization, and Transformation pathways.

Models: Structured imitations of a system's attributes and mechanisms to mimic appearance or functioning of systems, for example, the climate, the economy of a country, or a crop. Mathematical models assemble (many) variables and relations (often in a computer code) to simulate system functioning and performance for variations in parameters and inputs.

Computable General Equilibrium (CGE) Model: A class of economic models that use actual economic data (i.e., input/output data), simplify the characterization of economic behaviour, and solve the whole system numerically. CGE models specify all economic relationships in mathematical terms and predict the changes in variables such as prices, output and economic welfare resulting from a change in economic policies, given information about technologies and consumer preferences (Hertel, 1997). See also General equilibrium analysis.

Integrated Model: Integrated models explore the interactions between multiple sectors of the economy or components of particular systems, such as the energy system. In the context of transformation pathways, they refer to models that, at a minimum, include full and disaggregated representations of the energy system and its linkage to the overall economy that will allow for consideration of interactions among different elements of that system. Integrated models may also include representations of the full economy, land use and land use change (LUC), and the climate system. See also Integrated assessment.

Sectoral Model: In the context of this report, sectoral models address only one of the core sectors that are discussed in this report, such as buildings, industry, transport, energy supply, and Agriculture, Forestry and Other Land Use (AFOLU).

Montreal Protocol: The Montreal Protocol on Substances that Deplete the Ozone Layer was adopted in Montreal in 1987, and subse- quently adjusted and amended in London (1990), Copenhagen (1992), Vienna (1995), Montreal (1997) and Beijing (1999). It controls the consumption and production of chlorine- and bromine- containing chemicals that destroy stratospheric ozone $\left(\mathrm{O}_{3}\right)$, such as chlorofluorocarbons (CFCs), methyl chloroform, carbon tetrachloride and many others.

Multi-criteria analysis (MCA): Integrates different decision parameters and values without assigning monetary values to all parameters. Multi-criteria analysis can combine quantitative and qualitative information. Also referred to as multi-attribute analysis.

Multi-attribute analysis: See Multi-criteria analysis (MCA).

Multi-gas: Next to carbon dioxide $\left(\mathrm{CO}_{2}\right)$, there are other forcing components taken into account in, e. g., achieving reduction for a basket of greenhouse gas $(\mathrm{GHG})$ emissions $\left(\mathrm{CO}_{2}\right.$, methane $\left(\mathrm{CH}_{4}\right)$, nitrous oxide $\left(\mathrm{N}_{2} \mathrm{O}\right)$, and fluorinated gases) or stabilization of $\mathrm{CO}_{2}$-equivalent concentrations (multi-gas stabilization, including GHGs and aerosols).

Nationally Appropriate Mitigation Action (NAMA): Nationally Appropriate Mitigation Actions are a concept for recognizing and financing emission reductions by developing countries in a post-2012 climate regime achieved through action considered appropriate in a given national context. The concept was first introduced in the Bali Action Plan in 2007 and is contained in the Cancún Agreements.

Nitrogen oxides $\left(\mathrm{NO}_{\mathrm{x}}\right)$ : Any of several oxides of nitrogen.

Nitrous oxide $\left(\mathrm{N}_{2} \mathrm{O}\right)$ : One of the six greenhouse gases (GHGs) to be mitigated under the Kyoto Protocol. The main anthropogenic source of $\mathrm{N}_{2} \mathrm{O}$ is agriculture (soil and animal manure management), but important contributions also come from sewage treatment, fossil fuel combustion, and chemical industrial processes. $\mathrm{N}_{2} \mathrm{O}$ is also produced naturally from a wide variety of biological sources in soil and water, particularly microbial action in wet tropical forests. See also Global Warming Potential (GWP) and Annex II.9.1 for GWP values.

Non-Annex I Parties/countries: Non-Annex I Parties are mostly developing countries. Certain groups of developing countries are recognized by the Convention as being especially vulnerable to the adverse impacts of climate change, including countries with low-lying coastal areas and those prone to desertification and drought. Others, such as countries that rely heavily on income from fossil fuel production and commerce, feel more vulnerable to the potential economic impacts of climate change response measures. The Convention emphasizes activities that promise to answer the special needs and concerns of these vulnerable countries, such as investment, insurance, and technology transfer. See also Annex I Parties/countries.

Normative analysis: Analysis in which judgments about the desirability of various policies are made. The conclusions rest on value judgments as well as on facts and theories. See also Descriptive analysis. 
Ocean energy: Energy obtained from the ocean via waves, tidal ranges, tidal and ocean currents, and thermal and saline gradients.

Offset (in climate policy): A unit of $\mathrm{CO}_{2}$-equivalent emissions that is reduced, avoided, or sequestered to compensate for emissions occurring elsewhere.

Oil sands and oil shale: Unconsolidated porous sands, sandstone rock, and shales containing bituminous material that can be mined and converted to a liquid fuel. See also Unconventional fuels.

Overshoot pathways: Emissions, concentration, or temperature pathways in which the metric of interest temporarily exceeds, or 'overshoots', the long-term goal.

Ozone $\left(\mathrm{O}_{3}\right)$ : Ozone, the triatomic form of oxygen $\left(\mathrm{O}_{3}\right)$, is a gaseous atmospheric constituent. In the troposphere, it is created both naturally and by photochemical reactions involving gases resulting from human activities (smog). Tropospheric $\mathrm{O}_{3}$ acts as a greenhouse gas (GHG). In the stratosphere, it is created by the interaction between solar ultraviolet radiation and molecular oxygen $\left(\mathrm{O}_{2}\right)$. Stratospheric $\mathrm{O}_{3}$ plays a dominant role in the stratospheric radiative balance. Its concentration is highest in the $\mathrm{O}_{3}$ layer.

Paratransit: Denotes flexible passenger transportation, often but not only in areas with low population density, that does not follow fixed routes or schedules. Options include minibuses (matatus, marshrutka), shared taxis and jitneys. Sometimes paratransit is also called community transit.

Pareto optimum: A state in which no one's welfare can be increased without reducing someone else's welfare. See also Economic efficiency.

Particulate matter (PM): Very small solid particles emitted during the combustion of biomass and fossil fuels. PM may consist of a wide variety of substances. Of greatest concern for health are particulates of diameter less than or equal to 10 nanometers, usually designated as $\mathrm{PM}_{10}$. See also Aerosol.

Passive design: The word 'passive' in this context implies the ideal target that the only energy required to use the designed product or service comes from renewable sources.

Path dependence: The generic situation where decisions, events, or outcomes at one point in time constrain adaptation, mitigation, or other actions or options at a later point in time.

Payback period: Mostly used in investment appraisal as financial payback, which is the time needed to repay the initial investment by the returns of a project. A payback gap exists when, for example, private investors and micro-financing schemes require higher profitability rates from renewable energy (RE) projects than from fossil-fired proj- ects. Energy payback is the time an energy project needs to deliver as much energy as had been used for setting the project online. Carbon payback is the time a renewable energy (RE) project needs to deliver as much net greenhouse gas (GHG) savings (with respect to the fossil reference energy system) as its realization has caused GHG emissions from a perspective of lifecycle assessment (LCA) (including land use changes (LUC) and loss of terrestrial carbon stocks).

Perfluorocarbons (PFCs): One of the six types of greenhouse gases (GHGs) or groups of GHGs to be mitigated under the Kyoto Protocol. PFCs are by-products of aluminium smelting and uranium enrichment. They also replace chlorofluorocarbons (CFCS) in manufacturing semiconductors. See also Global Warming Potential (GWP) and Annex II.9.1 for GWP values.

Photovoltaic cells (PV): Electronic devices that generate electricity from light energy. See also Solar energy.

Policies (for mitigation of or adaptation to climate change): Policies are a course of action taken and/or mandated by a government, e. g., to enhance mitigation and adaptation. Examples of policies aimed at mitigation are support mechanisms for renewable energy (RE) supplies, carbon or energy taxes, fuel efficiency standards for automobiles. See also Measures.

Polluter pays principle (PPP): The party causing the pollution is responsible for paying for remediation or for compensating the damage.

\section{Positive analysis: See Descriptive analysis.}

Potential: The possibility of something happening, or of someone doing something in the future. Different metrics are used throughout this report for the quantification of different types of potentials, including the following:

Technical potential: Technical potential is the amount by which it is possible to pursue a specific objective through an increase in deployment of technologies or implementation of processes and practices that were not previously used or implemented. Quantification of technical potentials may take into account other than technical considerations, including social, economic and/or environmental considerations.

Precautionary principle: A provision under Article 3 of the United Nations Framework Convention on Climate Change (UNFCCC), stipulating that the Parties should take precautionary measures to anticipate, prevent, or minimize the causes of climate change and mitigate its adverse effects. Where there are threats of serious or irreversible damage, lack of full scientific certainty should not be used as a reason to postpone such measures, taking into account that policies and measures to deal with climate change should be cost-effective in order to ensure global benefits at the lowest possible cost. 
Precursors: Atmospheric compounds that are not greenhouse gases (GHGS) or aerosols, but that have an effect on GHG or aerosol concentrations by taking part in physical or chemical processes regulating their production or destruction rates.

\section{Pre-industrial: See Industrial Revolution.}

Present value: Amounts of money available at different dates in the future are discounted back to a present value, and summed to get the present value of a series of future cash flows. See also Discounting.

Primary production: All forms of production accomplished by plants, also called primary producers.

Primary energy: See Energy.

Private costs: Private costs are carried by individuals, companies or other private entities that undertake an action, whereas social costs include additionally the external costs on the environment and on society as a whole. Quantitative estimates of both private and social costs may be incomplete, because of difficulties in measuring all relevant effects.

Production-based accounting: Production-based accounting provides a measure of emissions released to the atmosphere for the production of goods and services by a certain entity (e.g., person, firm, country, or region). See also Consumption-based accounting.

Public good: Public goods are non-rivalrous (goods whose consumption by one consumer does not prevent simultaneous consumption by other consumers) and non-excludable (goods for which it is not possible to prevent people who have not paid for it from having access to it).

Purchasing power parity (PPP): The purchasing power of a currency is expressed using a basket of goods and services that can be bought with a given amount in the home country. International comparison of, for example, gross domestic products (GDP) of countries can be based on the purchasing power of currencies rather than on current exchange rates. PPP estimates tend to lower per capita GDP in industrialized countries and raise per capita GDP in developing countries. (PPP is also an acronym for polluter pays principle). See also Market exchange rate (MER) and Annex II.1.3 for the monetary conversion process applied throughout this report.

Radiation management: See Solar Radiation Management.

Radiative forcing: Radiative forcing is the change in the net, downward minus upward, radiative flux (expressed in $\mathrm{W} \mathrm{m}^{-2}$ ) at the tropopause or top of atmosphere due to a change in an external driver of climate change, such as, for example, a change in the concentration of carbon dioxide $\left(\mathrm{CO}_{2}\right)$ or the output of the sun. For the purposes of this report, radiative forcing is further defined as the change relative to the year 1750 and refers to a global and annual average value.

Rebound effect: Phenomena whereby the reduction in energy consumption or emissions (relative to a baseline) associated with the implementation of mitigation measures in a jurisdiction is offset to some degree through induced changes in consumption, production, and prices within the same jurisdiction. The rebound effect is most typically ascribed to technological energy efficiency (EE) improvements. See also Leakage.

Reducing Emissions from Deforestation and Forest Degradation (REDD): An effort to create financial value for the carbon stored in forests, offering incentives for developing countries to reduce emissions from forested lands and invest in low-carbon paths to sustainable development (SD). It is therefore a mechanism for mitigation that results from avoiding deforestation. REDD+ goes beyond reforestation and forest degradation, and includes the role of conservation, sustainable management of forests and enhancement of forest carbon stocks. The concept was first introduced in 2005 in the 11 th Session of the Conference of the Parties (COP) in Montreal and later given greater recognition in the 13th Session of the COP in 2007 at Bali and inclusion in the Bali Action Plan which called for "policy approaches and positive incentives on issues relating to reducing emissions to deforestation and forest degradation in developing countries (REDD) and the role of conservation, sustainable management of forests and enhancement of forest carbon stock in developing countries". Since then, support for REDD has increased and has slowly become a framework for action supported by a number of countries.

\section{Reference scenario: See Baseline/reference.}

Reforestation: Planting of forests on lands that have previously sustained forests but that have been converted to some other use. Under the United Nations Framework Convention on Climate Change (UNFCCC) and the Kyoto Protocol, reforestation is the direct humaninduced conversion of non-forested land to forested land through planting, seeding, and/or human-induced promotion of natural seed sources, on land that was previously forested but converted to nonforested land. For the first commitment period of the Kyoto Protocol, reforestation activities will be limited to reforestation occurring on those lands that did not contain forest on 31 December 1989.

For a discussion of the term forest and related terms such as afforestation, reforestation and deforestation, see the IPCC Report on Land Use, Land-Use Change and Forestry (IPCC, 2000). See also the Report on Definitions and Methodological Options to Inventory Emissions from Direct Human-induced Degradation of Forests and Devegetation of Other Vegetation Types (IPCC, 2003).

Renewable energy (RE): See Energy. 
Representative Concentration Pathways (RCPs): Scenarios that include time series of emissions and concentrations of the full suite of greenhouse gases (GHGS) and aerosols and chemically active gases, as well as land use/land cover (Moss et al., 2008). The word representative signifies that each RCP provides only one of many possible scenarios that would lead to the specific radiative forcing characteristics. The term pathway emphasizes that not only the long-term concentration levels are of interest, but also the trajectory taken over time to reach that outcome (Moss et al., 2010).

RCPs usually refer to the portion of the concentration pathway extending up to 2100, for which Integrated Assessment Models produced corresponding emission scenarios. Extended Concentration Pathways (ECPs) describe extensions of the RCPs from 2100 to 2500 that were calculated using simple rules generated by stakeholder consultations, and do not represent fully consistent scenarios.

Four RCPs produced from Integrated Assessment Models were selected from the published literature and are used in the present IPCC Assessment as a basis for the climate predictions and projections presented in WGI AR5 Chapters 11 to 14:

RCP2.6 One pathway where radiative forcing peaks at approximately $3 \mathrm{~W} \mathrm{~m}^{-2}$ before 2100 and then declines (the corresponding ECP assuming constant emissions after 2100);

RCP4.5 and RCP6.0 Two intermediate stabilization pathways in which radiative forcing is stabilized at approximately $4.5 \mathrm{~W} \mathrm{~m}^{-2}$ and $6.0 \mathrm{~W} \mathrm{~m}^{-2}$ after 2100 (the corresponding ECPs assuming constant concentrations after 2150);

RCP8.5 One high pathway for which radiative forcing reaches greater than $8.5 \mathrm{~W} \mathrm{~m}^{-2}$ by 2100 and continues to rise for some amount of time (the corresponding ECP assuming constant emissions after 2100 and constant concentrations after 2250).

For further description of future scenarios, see WGI AR5 Box 1.1. See also Baseline/reference, Climate prediction, Climate projection, Climate scenario, Shared socio-economic pathways, Socio-economic scenario, SRES scenarios, and Transformation pathway.

Reservoir: A component of the climate system, other than the atmosphere, which has the capacity to store, accumulate or release a substance of concern, for example, carbon, a greenhouse gas (GHG) or a precursor. Oceans, soils and forests are examples of reservoirs of carbon. Pool is an equivalent term (note that the definition of pool often includes the atmosphere). The absolute quantity of the substance of concern held within a reservoir at a specified time is called the stock. In the context of Carbon Dioxide Capture and Storage (CCS), this term is sometimes used to refer to a geological carbon dioxide $\left(\mathrm{CO}_{2}\right)$ storage location. See also Sequestration.
Resilience: The capacity of social, economic, and environmental systems to cope with a hazardous event or trend or disturbance, responding or reorganizing in ways that maintain their essential function, identity, and structure, while also maintaining the capacity for adaptation, learning, and transformation (Arctic Council, 2013).

Revegetation: A direct human-induced activity to increase carbon stocks on sites through the establishment of vegetation that covers a minimum area of 0.05 hectares and does not meet the definitions of afforestation and reforestation contained here (UNFCCC, 2002).

Risk: In this report, the term risk is often used to refer to the potential, when the outcome is uncertain, for adverse consequences on lives, livelihoods, health, ecosystems and species, economic, social and cultural assets, services (including environmental services), and infrastructure.

Risk assessment: The qualitative and/or quantitative scientific estimation of risks.

Risk management: The plans, actions, or policies to reduce the likelihood and/or consequences of a given risk.

Risk perception: The subjective judgment that people make about the characteristics and severity of a risk.

Risk tradeoff: The change in the portfolio of risks that occurs when a countervailing risk is generated (knowingly or inadvertently) by an intervention to reduce the target risk (Wiener and Graham, 2009). See also Adverse side-effect, and Co-benefit.

Risk transfer: The practice of formally or informally shifting the risk of financial consequences for particular negative events from one party to another.

Scenario: A plausible description of how the future may develop based on a coherent and internally consistent set of assumptions about key driving forces (e.g., rate of technological change (TC), prices) and relationships. Note that scenarios are neither predictions nor forecasts, but are useful to provide a view of the implications of developments and actions. See also Baseline/reference, Climate scenario, Emission scenario, Mitigation scenario, Representative Concentration Pathways (RCPS), Shared socio-economic pathways, Socioeconomic scenarios, SRES scenarios, Stabilization, and Transformation pathway.

Scope 1, Scope 2, and Scope 3 emissions: See Emissions.

Secondary energy: See Primary energy.

Sectoral Models: See Models.

Sensitivity analysis: Sensitivity analysis with respect to quantitative analysis assesses how changing assumptions alters the outcomes. For 
example, one chooses different values for specific parameters and reruns a given model to assess the impact of these changes on model output.

Sequestration: The uptake (i.e., the addition of a substance of concern to a reservoir) of carbon containing substances, in particular carbon dioxide $\left(\mathrm{CO}_{2}\right)$, in terrestrial or marine reservoirs. Biological sequestration includes direct removal of $\mathrm{CO}_{2}$ from the atmosphere through land-use change (LUC), afforestation, reforestation, revegetation, carbon storage in landfills, and practices that enhance soil carbon in agriculture (cropland management, grazing land management). In parts of the literature, but not in this report, (carbon) sequestration is used to refer to Carbon Dioxide Capture and Storage (CCS).

Shadow pricing: Setting prices of goods and services that are not, or are incompletely, priced by market forces or by administrative regulation, at the height of their social marginal value. This technique is used in cost-benefit analysis (CBA).

Shared socio-economic pathways (SSPs): Currently, the idea of SSPs is developed as a basis for new emissions and socio-economic scenarios. An SSP is one of a collection of pathways that describe alternative futures of socio-economic development in the absence of climate policy intervention. The combination of SSP-based socio-economic scenarios and Representative Concentration Pathway (RCP)based climate projections should provide a useful integrative frame for climate impact and policy analysis. See also Baseline/reference, Climate scenario, Emission scenario, Mitigation scenario, Scenario, SRES scenarios, Stabilization, and Transformation pathway.

Short-lived climate pollutant (SLCP): Pollutant emissions that have a warming influence on climate and have a relatively short lifetime in the atmosphere (a few days to a few decades). The main SLCPs are black carbon (BC) ('soot'), methane $\left(\mathrm{CH}_{4}\right)$ and some hydroflurorcarbons (HFCS) some of which are regulated under the Kyoto Protocol. Some pollutants of this type, including $\mathrm{CH}_{4}$, are also precursors to the formation of tropospheric ozone $\left(\mathrm{O}_{3}\right)$, a strong warming agent. These pollutants are of interest for at least two reasons. First, because they are short-lived, efforts to control them will have prompt effects on global warming-unlike long-lived pollutants that build up in the atmosphere and respond to changes in emissions at a more sluggish pace. Second, many of these pollutants also have adverse local impacts such as on human health.

Sink: Any process, activity or mechanism that removes a greenhouse gas (GHG), an aerosol, or a precursor of a GHG or aerosol from the atmosphere.

Smart grids: A smart grid uses information and communications technology to gather data on the behaviours of suppliers and consumers in the production, distribution, and use of electricity. Through automated responses or the provision of price signals, this information can then be used to improve the efficiency, reliability, economics, and sustainability of the electricity network.

Smart meter: A meter that communicates consumption of electricity or gas back to the utility provider.

Social cost of carbon (SCC): The net present value of climate damages (with harmful damages expressed as a positive number) from one more tonne of carbon in the form of carbon dioxide $\left(\mathrm{CO}_{2}\right)$, conditional on a global emissions trajectory over time.

Social costs: See Private costs.

Socio-economic scenario: A scenario that describes a possible future in terms of population, gross domestic product (GDP), and other socioeconomic factors relevant to understanding the implications of climate change. See also Baseline/reference, Climate scenario, Emission scenario, Mitigation scenario, Representative Concentration Pathways (RCPS), Scenario, Shared socio-economic pathways, SRES scenarios, Stabilization, and Transformation pathway.

Solar energy: Energy from the sun. Often the phrase is used to mean energy that is captured from solar radiation either as heat, as light that is converted into chemical energy by natural or artificial photosynthesis, or by photovoltaic panels and converted directly into electricity.

Solar Radiation Management (SRM): Solar Radiation Management refers to the intentional modification of the earth's shortwave radiative budget with the aim to reduce climate change according to a given metric (e.g., surface temperature, precipitation, regional impacts, etc.). Artificial injection of stratospheric aeroso/s and cloud brightening are two examples of SRM techniques. Methods to modify some fastresponding elements of the longwave radiative budget (such as cirrus clouds), although not strictly speaking SRM, can be related to SRM. SRM techniques do not fall within the usual definitions of mitigation and adaptation (IPCC, 2012, p. 2). See also Carbon Dioxide Removal (CDR) and Geoengineering.

Source: Any process, activity or mechanism that releases a greenhouse gas (GHG), an aerosol or a precursor of a GHG or aerosol into the atmosphere. Source can also refer to, e.g., an energy source.

Spill-over effect: The effects of domestic or sector mitigation measures on other countries or sectors. Spill-over effects can be positive or negative and include effects on trade, (carbon) leakage, transfer of innovations, and diffusion of environmentally sound technology and other issues.

SRES scenarios: SRES scenarios are emission scenarios developed by Nakićenović and Swart (2000) and used, among others, as a basis for some of the climate projections shown in Chapters 9 to 11 of IPCC (2001) and Chapters 10 and 11 of IPCC (2007) as well as WGI AR5. The 
following terms are relevant for a better understanding of the structure and use of the set of SRES scenarios:

Scenario family: Scenarios that have a similar demographic, societal, economic and technical change storyline. Four scenario families comprise the SRES scenario set: A1, A2, B1, and B2.

Illustrative Scenario: A scenario that is illustrative for each of the six scenario groups reflected in the Summary for Policymakers of Nakićenović and Swart (2000). They include four revised marker scenarios for the scenario groups A1B, A2, B1, B2, and two additional scenarios for the A1FI and A1T groups. All scenario groups are equally sound.

Marker Scenario: A scenario that was originally posted in draft form on the SRES website to represent a given scenario family. The choice of markers was based on which of the initial quantifications best reflected the storyline, and the features of specific models. Markers are no more likely than other scenarios, but are considered by the SRES writing team as illustrative of a particular storyline. They are included in revised form in Nakićenović and Swart (2000). These scenarios received the closest scrutiny of the entire writing team and via the SRES open process. Scenarios were also selected to illustrate the other two scenario groups.

Storyline: A narrative description of a scenario (or family of scenarios), highlighting the main scenario characteristics, relationships between key driving forces and the dynamics of their evolution.

See also Baseline/reference, Climate scenario, Emission scenario, Mitigation scenario, Representative Concentration Pathways (RCPS), Shared socio-economic pathways, Socio-economic scenario, Stabilization, and Transformation pathway.

Stabilization (of GHG or $\mathrm{CO}_{2}$-equivalent concentration): A state in which the atmospheric concentrations of one greenhouse gas (GHG) (e. g., carbon dioxide) or of a $\mathrm{CO}_{2}$-equivalent basket of GHGs (or a combination of GHGs and aerosols) remains constant over time.

Standards: Set of rules or codes mandating or defining product performance (e. g., grades, dimensions, characteristics, test methods, and rules for use). Product, technology or performance standards establish minimum requirements for affected products or technologies. Standards impose reductions in greenhouse gas (GHG) emissions associated with the manufacture or use of the products and/or application of the technology.

Stratosphere: The highly stratified region of the atmosphere above the troposphere extending from about $10 \mathrm{~km}$ (ranging from $9 \mathrm{~km}$ at high latitudes to $16 \mathrm{~km}$ in the tropics on average) to about $50 \mathrm{~km}$ altitude.

Structural change: Changes, for example, in the relative share of gross domestic product (GDP) produced by the industrial, agricultural, or services sectors of an economy, or more generally, systems transformations whereby some components are either replaced or potentially substituted by other components.

Subsidiarity: The principle that decisions of government (other things being equal) are best made and implemented, if possible, at the lowest most decentralized level, that is, closest to the citizen. Subsidiarity is designed to strengthen accountability and reduce the dangers of making decisions in places remote from their point of application. The principle does not necessarily limit or constrain the action of higher orders of government, but merely counsels against the unnecessary assumption of responsibilities at a higher level.

Sulphur hexafluoride $\left(\mathrm{SF}_{6}\right)$ : One of the six types of greenhouse gases (GHGs) to be mitigated under the Kyoto Protocol. $\mathrm{SF}_{6}$ is largely used in heavy industry to insulate high-voltage equipment and to assist in the manufacturing of cable-cooling systems and semi-conductors. See Global Warming Potential (GWP) and Annex II.9.1 for GWP values.

Sustainability: A dynamic process that guarantees the persistence of natural and human systems in an equitable manner.

Sustainable development (SD): Development that meets the needs of the present without compromising the ability of future generations to meet their own needs (WCED, 1987).

\section{Technical potential: See Potential.}

Technological change (TC): Economic models distinguish autonomous (exogenous), endogenous, and induced TC.

Autonomous (exogenous) technological change: Autonomous (exogenous) technological change is imposed from outside the model (i.e., as a parameter), usually in the form of a time trend affecting factor and/or energy productivity and therefore energy demand and/or economic growth.

Endogenous technological change: Endogenous technological change is the outcome of economic activity within the model (i. e., as a variable) so that factor productivity or the choice of technologies is included within the model and affects energy demand and/or economic growth.

Induced technological change: Induced technological change implies endogenous technological change but adds further changes induced by policies and measures, such as carbon taxes triggering research and development efforts.

Technological learning: See Learning curve/rate.

Technological/knowledge spillovers: Any positive externality that results from purposeful investment in technological innovation or development (Weyant and Olavson, 1999). 
Territorial emissions: See Emissions.

Trace gas: A minor constituent of the atmosphere, next to nitrogen and oxygen that together make up $99 \%$ of all volume. The most important trace gases contributing to the greenhouse effect are carbon dioxide $\left(\mathrm{CO}_{2}\right)$, ozone $\left(\mathrm{O}_{3}\right)$, methane $\left(\mathrm{CH}_{4}\right)$, nitrous oxide $\left(\mathrm{N}_{2} \mathrm{O}\right)$, perfluorocarbons (PFCS), chlorofluorocarbons (CFCS), hydrofluorocarbons (HFCS), sulphur hexafluoride (SF $)$ and water vapour $\left(\mathrm{H}_{2} \mathrm{O}\right)$.

Tradable (green) certificates scheme: A market-based mechanism to achieve an environmentally desirable outcome (renewable energy (RE) generation, energy efficiency (EE) requirements) in a cost-effective way by allowing purchase and sale of certificates representing under and over-compliance respectively with a quota.

Tradable (emission) permit: See Emission permit.

Tradable quota system: See Emissions trading.

Transaction costs: The costs that arise from initiating and completing transactions, such as finding partners, holding negotiations, consulting with lawyers or other experts, monitoring agreements, or opportunity costs, such as lost time or resources (Michaelowa et al., 2003).

Transformation pathway: The trajectory taken over time to meet different goals for greenhouse gas (GHG) emissions, atmospheric concentrations, or global mean surface temperature change that implies a set of economic, technological, and behavioural changes. This can encompass changes in the way energy and infrastructure is used and produced, natural resources are managed, institutions are set up, and in the pace and direction of technological change (TC). See also Baseline/reference, Climate scenario, Emission scenario, Mitigation scenario, Representative Concentration Pathways (RCPS), Scenario, Shared socio-economic pathways, Socio-economic scenarios, SRES scenarios, and Stabilization.

Transient climate response: See Climate sensitivity.

Transit oriented development (TOD): Urban development within walking distance of a transit station, usually dense and mixed with the character of a walkable environment.

Troposphere: The lowest part of the atmosphere, from the surface to about $10 \mathrm{~km}$ in altitude at mid-latitudes (ranging from $9 \mathrm{~km}$ at high latitudes to $16 \mathrm{~km}$ in the tropics on average), where clouds and weather phenomena occur. In the troposphere, temperatures generally decrease with height. See also Stratosphere.

Uncertainty: A cognitive state of incomplete knowledge that can result from a lack of information or from disagreement about what is known or even knowable. It may have many types of sources, from imprecision in the data to ambiguously defined concepts or terminol- ogy, or uncertain projections of human behaviour. Uncertainty can therefore be represented by quantitative measures (e.g., a probability density function) or by qualitative statements (e.g., reflecting the judgment of a team of experts) (see Moss and Schneider, 2000; Manning et al., 2004; Mastrandrea et al., 2010). See also Agreement, Evidence, Confidence and Likelihood.

Unconventional resources: A loose term to describe fossil fuel reserves that cannot be extracted by the well-established drilling and mining processes that dominated extraction of coal, gas, and oil throughout the 20th century. The boundary between conventional and unconventional resources is not clearly defined. Unconventional oils include oil shales, tar sands/bitumen, heavy and extra heavy crude oils, and deep-sea oil occurrences. Unconventional natural gas includes gas in Devonian shales, tight sandstone formations, geopressured aquifers, coal-bed gas, and methane $\left(\mathrm{CH}_{4}\right)$ in clathrate structures (gas hydrates) (Rogner, 1997).

United Nations Framework Convention on Climate Change (UNFCCC): The Convention was adopted on 9 May 1992 in New York and signed at the 1992 Earth Summit in Rio de Janeiro by more than 150 countries and the European Community. Its ultimate objective is the 'stabilisation of greenhouse gas concentrations in the atmosphere at a level that would prevent dangerous anthropogenic interference with the climate system'. It contains commitments for all Parties under the principle of 'common but differentiated responsibilities'. Under the Convention, Parties included in Annex / aimed to return greenhouse gas (GHG) emissions not controlled by the Montreal Protocol to 1990 levels by the year 2000. The convention entered in force in March 1994. In 1997, the UNFCCC adopted the Kyoto Protocol.

\section{Urban heat island: See Heat island.}

Verified Emissions Reductions: Emission reductions that are verified by an independent third party outside the framework of the United Nations Framework Convention on Climate Change (UNFCCC) and its Kyoto Protocol. Also called 'Voluntary Emission Reductions'.

Volatile Organic Compounds (VOCs): Important class of organic chemical air pollutants that are volatile at ambient air conditions. Other terms used to represent VOCs are hydrocarbons (HCs), reactive organic gases (ROGs) and non-methane volatile organic compounds (NMVOCs). NMVOCs are major contributors-together with nitrogen oxides $\left(N O_{x}\right)$, and carbon monoxide $(\mathrm{CO})$ - to the formation of photochemical oxidants such as ozone $\left(\mathrm{O}_{3}\right)$.

Voluntary action: Informal programmes, self-commitments, and declarations, where the parties (individual companies or groups of companies) entering into the action set their own targets and often do their own monitoring and reporting. 
Voluntary agreement (VA): An agreement between a government authority and one or more private parties to achieve environmental objectives or to improve environmental performance beyond compliance with regulated obligations. Not all voluntary agreements are truly voluntary; some include rewards and/or penalties associated with joining or achieving commitments.

Voluntary Emission Reductions: See Verified Emissions Reductions.

Watts per square meter ( $\mathrm{W} \mathrm{m}^{-2}$ ): See Radiative forcing.
Wind energy: Kinetic energy from air currents arising from uneven heating of the earth's surface. A wind turbine is a rotating machine for converting the kinetic energy of the wind to mechanical shaft energy to generate electricity. A windmill has oblique vanes or sails and the mechanical power obtained is mostly used directly, for example, for water pumping. A wind farm, wind project, or wind power plant is a group of wind turbines interconnected to a common utility system through a system of transformers, distribution lines, and (usually) one substation. 


\section{Acronyms and chemical symbols}

\begin{tabular}{|c|c|}
\hline AAU & Assigned Amount Unit \\
\hline ADB & Asian Development Bank \\
\hline AfDB & African Development Bank \\
\hline AFOLU & Agriculture, Forestry and Other Land Use \\
\hline AME & Asian Modeling Exercise \\
\hline AMPERE & $\begin{array}{l}\text { Assessment of Climate Change Mitigation Pathways } \\
\text { and Evaluation of the Robustness of Mitigation Cost } \\
\text { Estimates }\end{array}$ \\
\hline AOSIS & Alliance of Small Island States \\
\hline APEC & Asia-Pacific Economic Cooperation \\
\hline AR4 & IPCC Fourth Assessment Report \\
\hline ASEAN & Association of Southeast Asian Nations \\
\hline ASIA & Non-OECD Asia \\
\hline BAMs & Border adjustment measures \\
\hline BAT & Best available technology \\
\hline BAU & Business-as-usual \\
\hline BC & Black carbon \\
\hline BECCS & Bioenergy with carbon dioxide capture and storage \\
\hline BEVs & Battery electric vehicles \\
\hline BNDES & Brazilian Development Bank \\
\hline BOD & Biochemical Oxygen Demand \\
\hline BRT & Bus rapid transit \\
\hline C & Carbon \\
\hline $\mathrm{C} 40$ & C40 Cities Climate Leadership Group \\
\hline CBA & Cost-benefit analysis \\
\hline CBD & Convention on Biological Diversity \\
\hline CBD & Central business district \\
\hline CCA & Climate Change Agreement \\
\hline CCE & Cost of conserved energy \\
\hline $\mathrm{CCL}$ & Climate Change Levy \\
\hline CCS & Carbon dioxide capture and storage \\
\hline CDM & Clean Development Mechanism \\
\hline CDR & Carbon dioxide removal \\
\hline CEA & Cost-effectiveness analysis \\
\hline CERs & Certified Emissions Reductions \\
\hline CFCs & Chlorofluorocarbons \\
\hline CGE & Computable general equilibrium \\
\hline $\mathrm{CH}_{4}$ & Methane \\
\hline CHP & Combined heat and power \\
\hline CIFs & Climate Investment Funds \\
\hline CMIP & Coupled Model Intercomparison Project \\
\hline CNG & Compressed natural gas \\
\hline $\mathrm{CO}$ & Carbon monoxide \\
\hline $\mathrm{CO}_{2}$ & Carbon dioxide \\
\hline $\mathrm{CO}_{2} \mathrm{eq}$ & Carbon dioxide-equivalent, $\mathrm{CO}_{2}$-equivalent \\
\hline COD & Chemical oxygen demand \\
\hline COP & Conference of the Parties \\
\hline CRF & Capital recovery factor \\
\hline CSP & Concentrated solar power \\
\hline CTCN & Climate Technology Centre and Network \\
\hline
\end{tabular}

\begin{tabular}{|c|c|}
\hline DAC & Direct air capture \\
\hline DAC & Development Assistance Committee \\
\hline DALYs & Disability-adjusted life years \\
\hline DANN & Designated National Authority \\
\hline DCs & Developing countries \\
\hline DRI & Direct reduced iron \\
\hline DSM & Demand-side management \\
\hline EAF & Electric arc furnace \\
\hline EAS & East Asia \\
\hline ECA & Economic Commission for Africa \\
\hline ECN & Energy Research Center of the Netherlands \\
\hline ECOWAS & Economic Community of West African States \\
\hline EDGAR & Emissions Database for Global Atmospheric Research \\
\hline EE & Energy efficiency \\
\hline EIA & U.S. Energy Information Administration \\
\hline EITs & Economies in Transition \\
\hline EMF & Energy Modeling Forum \\
\hline EPA & U.S. Environmental Protection Agency \\
\hline EPC & Energy performance contracting \\
\hline ERU & Emissions reduction unit \\
\hline ESCOs & Energy service companies \\
\hline ETS & Emissions Trading System \\
\hline EU & European Union \\
\hline EU ETS & European Union Emissions Trading Scheme \\
\hline EVs & Electric vehicles \\
\hline F-gases & Fluorinated gases \\
\hline FAO & $\begin{array}{l}\text { Food and Agriculture Organization of the United } \\
\text { Nations }\end{array}$ \\
\hline FAQ & Frequently asked questions \\
\hline FAR & IPCC First Assessment Report \\
\hline FCVs & Fuel cell vehicles \\
\hline FDI & Foreign Direct Investment \\
\hline FE & Final energy \\
\hline FEEM & Fondazione Eni Enrico Mattei \\
\hline FF\&I & Fossil fuel and industrial \\
\hline FIT & Feed-in tariff \\
\hline FOLU & Forestry and Other Land Use \\
\hline FSF & Fast-start Finance \\
\hline G20 & Group of Twenty Finance Ministers \\
\hline G8 & Group of Eight Finance Ministers \\
\hline GATT & General Agreement on Tariffs and Trade \\
\hline GCAM & Global Change Assessment Model \\
\hline GCF & Green Climate Fund \\
\hline GCM & General Circulation Model \\
\hline GDP & Gross domestic product \\
\hline GEA & Global Energy Assessment \\
\hline GEF & Global Environment Facility \\
\hline GHG & Greenhouse gas \\
\hline GNE & Gross national expenditure \\
\hline GSEP & Global Superior Energy Performance Partnership \\
\hline GTM & Global Timber Model \\
\hline GTP & Global Temperature Change Potential \\
\hline GWP & Global Warming Potential \\
\hline
\end{tabular}




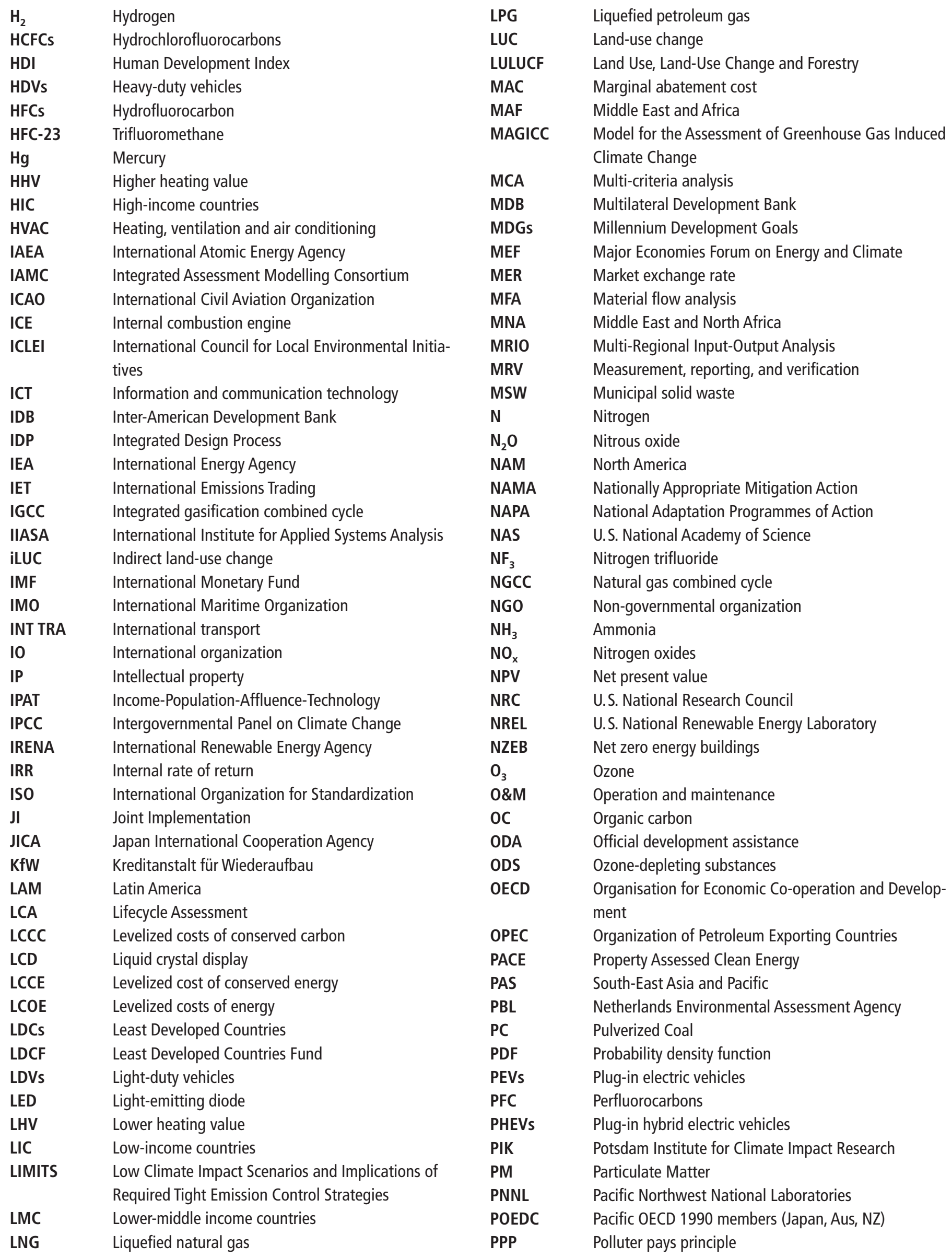




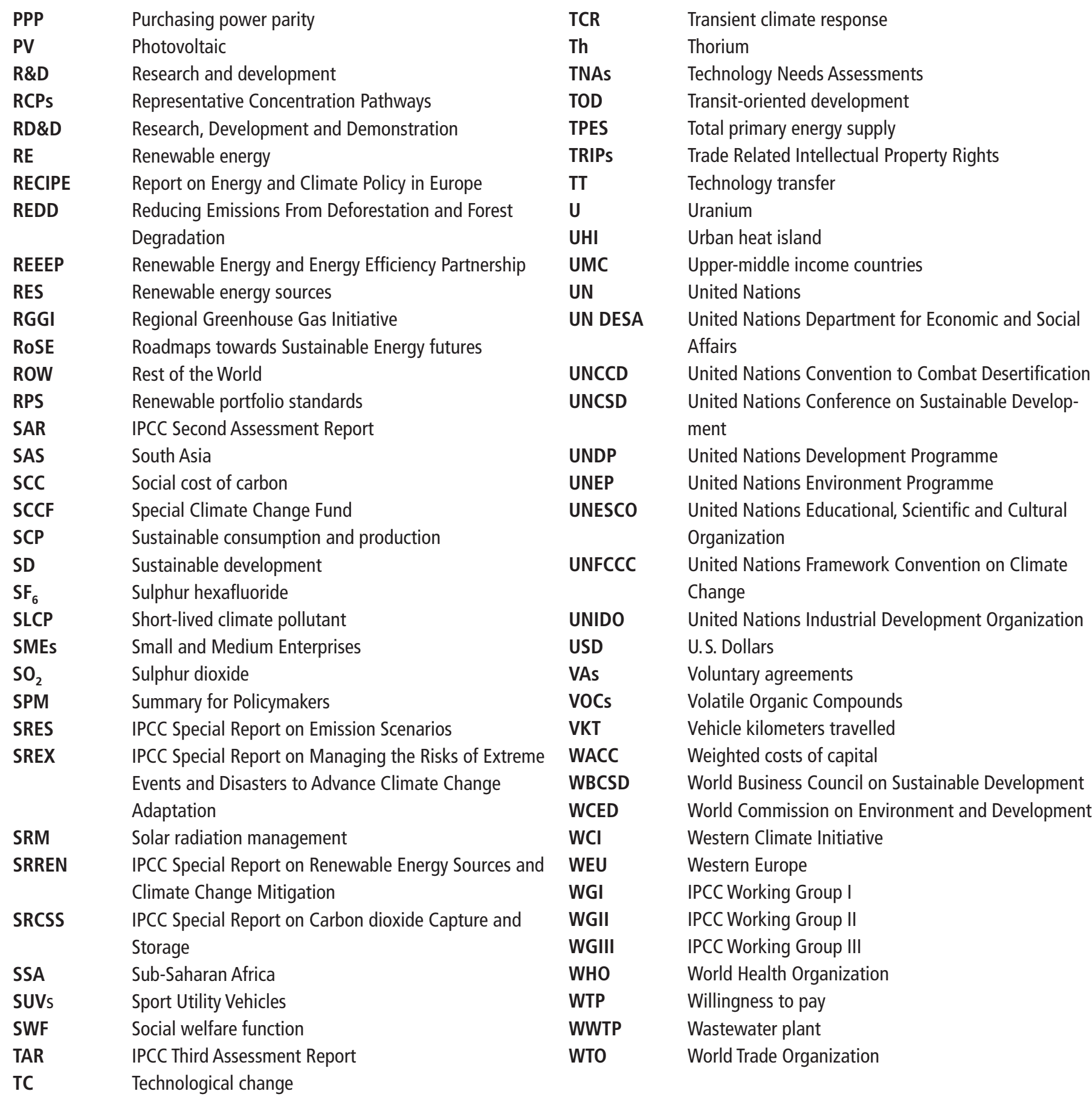




\section{References}

United Nations Secretary General's Advisory Group on Energy and Climate (AGECC) (2010). Energy for a Sustainable Future. New York, NY, USA.

Arctic Council (2013). Glossary of terms. In: Arctic Resilience Interim Report 2013. Stockholm Environment Institute and Stockholm Resilience Centre, Stockholm, Sweden.

Brunner, P.H. and H. Rechberger (2004). Practical handbook of material flow analysis. The International Journal of Life Cycle Assessment, 9(5), 337-338.

Cobo, J.R.M. (1987). Study of the problem of discrimination against indigenous populations. Sub-commission on Prevention of Discrimination and Protection of Minorities. New York: United Nations, 1987.

Ehrlich, P.R. and J.P. Holdren (1971). Impact of population growth. Science, 171(3977), 1212-1217.

Food and Agricultural Organization of the United Nations (FAO) (2000). State of food insecurity in the world 2000. Rome, Italy.

Hertel, T.T.W. (1997). Global trade analysis: modeling and applications. T.W. Hertel (Ed.). Cambridge University Press, Cambridge, United Kingdom.

Heywood, V.H. (ed.) (1995). The Global Biodiversity Assessment. United Nations Environment Programme. Cambridge University Press, Cambridge, United Kingdom.

IPCC (1992). Climate Change 1992: The Supplementary Report to the IPCC Scientific Assessment [Houghton, J.T., B.A. Callander, and S.K. Varney (eds.)]. Cambridge University Press, Cambridge, United Kingdom and New York, NY, USA, $116 \mathrm{pp}$.

IPCC (1996). Climate Change 1995: The Science of Climate Change. Contribution of Working Group I to the Second Assessment Report of the Intergovernmental Panel on Climate Change [Houghton, J.T., L.G. Meira Filho, B.A. Callander, N. Harris, A. Kattenberg, and K. Maskell (eds.)]. Cambridge University Press, Cambridge, United Kingdom and New York, NY, USA, 572 pp.

IPCC (2000). Land Use, Land-Use Change, and Forestry. Special Report of the Intergovernmental Panel on Climate Change [Watson, R.T., I.R. Noble, B. Bolin, N.H. Ravindranath, D. J. Verardo, and D. J. Dokken (eds.)]. Cambridge University Press, Cambridge, United Kingdom and New York, NY, USA, 377 pp.

IPCC (2001). Climate Change 2001: The Scientific Basis. Contribution of Working Group I to the Third Assessment Report of the Intergovernmental Panel on Climate Change [Houghton, J.T., Y. Ding, D. J. Griggs, M. Noguer, P. J. van der Linden, X. Dai, K. Maskell, and C.A. Johnson (eds.)]. Cambridge University Press, Cambridge, United Kingdom and New York, NY, USA, 881 pp.

IPCC (2003). Definitions and Methodological Options to Inventory Emissions from Direct Human-Induced Degradation of Forests and Devegetation of Other Vegetation Types [Penman, J., M. Gytarsky, T. Hiraishi, T. Krug, D. Kruger, R. Pipatti, L. Buendia, K. Miwa, T. Ngara, K. Tanabe, and F. Wagner (eds.)]. The Institute for Global Environmental Strategies (IGES), Japan, 32 pp.

IPCC (2006). 2006 IPCC Guidelines for National Greenhouse Gas Inventories, Prepared by the National Greenhouse Gas Inventories Programme [Eggleston H.S., L. Buendia, K. Miwa, T. Ngara and K. Tanabe K. (eds.)]. The Institute for Global Environmental Strategies (IGES), Japan.
IPCC (2007). Climate Change 2007: The Physical Science Basis. Contribution of Working Group I to the Fourth Assessment Report of the Intergovernmental Panel on Climate Change [Solomon, S., D. Qin, M. Manning, Z. Chen, M. Marquis, K. B. Averyt, M. Tignor, and H. L. Miller (eds.)]. Cambridge University Press, Cambridge, United Kingdom and New York, NY, USA, 996 pp.

IPCC (2012). Meeting Report of the Intergovernmental Panel on Climate Change Expert Meeting on Geoengineering [0. Edenhofer, R. Pichs-Madruga, Y. Sokona, C. Field, V. Barros, T.F. Stocker, Q. Dahe, J. Minx, K. Mach, G.-K. Plattner, S. Schlömer, G. Hansen, and M. Mastrandrea (eds.)]. IPCC Working Group III Technical Support Unit, Potsdam Institute for Climate Impact Research, Potsdam, Germany, 99 pp.

Manning, M.R., M. Petit, D. Easterling, J. Murphy, A. Patwardhan, H-H. Rogner, R. Swart, and G. Yohe (eds.) (2004). IPCC Workshop on Describing Scientific Uncertainties in Climate Change to Support Analysis of Risk of Options. Workshop Report. Intergovernmental Panel on Climate Change, Geneva, Switzerland.

Mastrandrea, M.D., C. B. Field, T. F. Stocker, O. Edenhofer, K. L. Ebi, D. J. Frame, H. Held, E. Kriegler, K.J. Mach, P.R. Matschoss, G.-K. Plattner, G.W. Yohe, and F.W. Zwiers (2010). Guidance Note for Lead Authors of the IPCC Fifth Assessment Report on Consistent Treatment of Uncertainties. Intergovernmental Panel on Climate Change (IPCC). Published online at: http://www.ipcc-wg2. gov/meetings/CGCs/index.html\#UR

Michaelowa, A., M. Stronzik., F. Eckermann, and A. Hunt (2003). Transaction costs of the Kyoto Mechanisms. Climate policy, 3(3), 261-278.

Millennium Ecosystem Assessment (MEA) (2005). Ecosystems and Human Wellbeing: Current States and Trends. World Resources Institute, Washington, D.C. [Appendix D, p. 893].

Moss, R., and S. Schneider (2000). Uncertainties in the IPCC TAR: Recommendations to Lead Authors for More Consistent Assessment and Reporting. In: IPCC Supporting Material: Guidance Papers on Cross Cutting Issues in the Third Assessment Report of the IPCC [Pachauri, R., T. Taniguchi, and K. Tanaka (eds.)]. Intergovernmental Panel on Climate Change, Geneva, Switzerland, pp. 33-51.

Moss, R., M. Babiker, S. Brinkman, E. Calvo, T. Carter, J. Edmonds, I. Elgizouli, S. Emori, L. Erda, K. Hibbard, R. Jones, M. Kainuma, J. Kelleher, J.F. Lamarque, M. Manning, B. Matthews, J. Meehl, L. Meyer, J. Mitchell, N. Nakicenovic, B. O'Neill, R. Pichs, K. Riahi, S. Rose, P. Runci, R. Stouffer, D. van Vuuren, J. Weyant, T. Wilbanks, J.P. van Ypersele, and M. Zurek (2008). Towards new scenarios for analysis of emissions, climate change, impacts and response strategies. Intergovernmental Panel on Climate Change, Geneva, Switzerland, $132 \mathrm{pp}$.

Moss, R., J.A. Edmonds, K.A. Hibbard, M.R. Manning, S. K. Rose, D.P. van Vuuren, T.R. Carter, S. Emori, M. Kainuma, T. Kram, G.A. Meehl, J.F.B. Mitchell, N. Nakicenovic, K. Riahi, S. J. Smith, R.J. Stouffer, A. M. Thomson, J.P. Weyant, and T.J. Wilbanks (2010). The next generation of scenarios for climate change research and assessment. Nature, 463, 747-756.

Nakićenović, N. and R. Swart (eds.) (2000). Special Report on Emissions Scenarios. A Special Report of Working Group III of the Intergovernmental Panel on Climate Change. Cambridge University Press, Cambridge, United Kingdom and New York, NY, USA, 599 pp.

Rogner, H.H. (1997). An assessment of world hydrocarbon resources. Annual review of energy and the environment, 22(1), 217-262. 
UNFCCC (2000). Report on the Conference of the Parties on its Seventh Session, held at Marrakesh from 29 October to 10 November 2001. Addendum. Part Two: Action Taken by the Conference of the Parties. (FCCC/CP/2001/13/Add.1).

United Nations Convention to Combat Desertification (UNCCD) (1994). Article 1: Use of terms. United Nations Convention to Combat Desertification. 17 June 1994: Paris, France.

Weyant, J.P. and T. Olavson (1999). Issues in modeling induced technological change in energy, environmental, and climate policy. Environmental Modeling \& Assessment, $4(2-3), 67-85$.
World Business Council on Sustainable Development (WBCSD) and World Resources Institute (WRI). (2004). The Greenhouse Gas Protocol - A Corporate Accounting and Reporting Standard. Geneva and Washington, DC.

Wiedmann, T. and J. Minx (2007). A definition of'carbon footprint. Ecological economics research trends, 1, 1-11.

Wiener, J.B. and J.D. Graham (2009). Risk vs. risk: Tradeoffs in protecting health and the environment. Harvard University Press, Cambridge, MA, USA.

World Commission on Environment and Development (WCED) (1987). Our Common Future. Oxford University Press, Oxford, United Kingdom 
